OPEN

\title{
KDIGO Clinical Practice Guideline on the Evaluation and Care of Living Kidney Donors
}

Krista L. Lentine, MD, PhD, ${ }^{1}$ Bertram L. Kasiske, MD, ${ }^{2}$ Andrew S. Levey, MD, ${ }^{3}$ Patricia L. Adams, MD, ${ }^{4}$ Josefina Alberú, MD, ${ }^{5}$ Mohamed A. Bakr, MD, ${ }^{6}$ Lorenzo Gallon, MD, ${ }^{7}$ Catherine A. Garvey, RN, ${ }^{8}$

Sandeep Guleria, MBBS, MS, DNB, ${ }^{9}$ Philip Kam-Tao Li, MD, ${ }^{10}$ Dorry L. Segev, MD, PhD, ${ }^{11}$ Sandra J. Taler, MD, ${ }^{12}$ Kazunari Tanabe, MD, PhD, ${ }^{13}$ Linda Wright, MHSc, MSW, ${ }^{14}$ Martin G. Zeier, MD, ${ }^{15}$ Michael Cheung, MA, ${ }^{16}$ and Amit X. Garg, MD, PhD ${ }^{17}$

Abstract: The 2017 Kidney Disease: Improving Global Outcomes (KDIGO) Clinical Practice Guideline on the Evaluation and Care of Living Kidney Donors is intended to assist medical professionals who evaluate living kidney donor candidates and provide care before, during and after donation. The guideline development process followed the Grades of Recommendation Assessment, Development, and Evaluation (GRADE) approach and guideline recommendations are based on systematic reviews of relevant studies that included critical appraisal of the quality of the evidence and the strength of recommendations. However, many recommendations, for which there was no evidence or no systematic search for evidence was undertaken by the Evidence Review Team, were issued as ungraded expert opinion recommendations. The guideline work group concluded that a comprehensive approach to risk assessment should replace decisions based on assessments of single risk factors in isolation. Original data analyses were undertaken to produce a "proof-in-concept" risk-prediction model for kidney failure to support a framework for quantitative risk assessment in the donor candidate evaluation and defensible shared decision making. This framework is grounded in the simultaneous consideration of each candidate's profile of demographic and health characteristics. The processes and framework for the donor candidate evaluation are presented, along with recommendations for optimal care before, during, and after donation. Limitations of the evidence are discussed, especially regarding the lack of definitive prospective studies and clinical outcome trials. Suggestions for future research, including the need for continued refinement of long-term risk prediction and novel approaches to estimating donation-attributable risks, are also provided.

(Transplantation 2017;101(8S): S1-S109)

In citing this document, the following format should be used: Kidney Disease: Improving Global Outcomes (KDIGO) Living Kidney Donor Work Group. KDIGO Clinical Practice Guideline on the Evaluation and Care of Living Kidney Donors. Transplantation 2017; 101(Suppl 8S):S1-S109.

Received 10 February 2017.

Accepted 20 March 2017.

${ }^{1}$ Saint Louis University School of Medicine, St. Louis, MO.

${ }^{2}$ Hennepin County Medical Center, Minneapolis, MN.

${ }^{3}$ Tufts Medical Center, Boston, MA.

${ }^{4}$ Wake Forest School of Medicine, Winston-Salem, NC.

${ }^{5}$ Instituto Nacional de Ciencias Médicas y Nutrición Salvador Zubirán, Mexico City, Mexico.

${ }^{6}$ Mansoura University Mansoura, Egypt.

${ }^{7}$ Northwestern University, Chicago, IL.

${ }^{8}$ University of Minnesota, Minneapolis, MN.

${ }^{9}$ Indraprastha Apollo Hospitals, New Delhi, India.

${ }^{10}$ Chinese University of Hong Kong, Hong Kong, China.

11 Johns Hopkins University, School of Medicine, Baltimore, MD.

${ }^{12}$ Mayo Clinic, Rochester, MN.

${ }^{13}$ Tokyo Women's Medical University, Tokyo, Japan.

${ }^{14}$ University of Toronto, Toronto, Canada.

${ }^{15}$ University Hospital Heidelberg, Heidelberg, Germany.

${ }^{16} \mathrm{KDIGO}$, Brussels, Belgium.

\footnotetext{
${ }^{17}$ Western University London, Canada.

Guideline Cochairs, K.L.L, A.X.G., contributed equally.

This Clinical Practice Guideline is based upon systematic literature searches last conducted in September 2014 supplemented with additional evidence through January 2017. It is designed to assist decision making. It is not intended to define a standard of care, and should not be interpreted as prescribing an exclusive course of management. Variations in practice will inevitably and appropriately occur when considering the needs of individual patients, available resources, and limitations unique to an institution or type of practice. Healthcare professionals using these recommendations should decide how to apply them to their own clinical practece.

Kidney Disease: Improving Global Outcomes (KD/GO) makes every effort to avoid any actual or reasonably perceived conflicts of interest that may arise from an outside relationship or a personal, professional, or business interest of a member of the work group. All members of the work group are required to complete, sign, and submit a disclosure and attestation form showing all such relationships that might be perceived as or are actual conflicts of interest. This document is updated annually and information is adjusted accordingly. All reported information is published in its entirety at the end of the document and is kept on file at KDIGO.
}

Disclosure and funding information can be found in the Appendix on page S106. Correspondence: Krista L. Lentine, MD, PhD (lentinek@slu.edu). 
Supplemental digital content (SDC) is available for this article. Direct URL citations appear in the printed text, and links to the digital files are provided in the HTML text of this article on the journal's Web site (www.transplantjournal.com).

Copyright (C) 2017 KDIGO. This is an open-access article distributed under the terms of the Creative Commons Attribution-Non Commercial-No Derivatives License 4.0
(CCBY-NC-ND), where it is permissible to download and share the work provided it is properly cited. The work cannot be changed in any way or used commercially without permission from the journal.

ISSN: 0041-1337/17/10108-00S1

DOI: $10.1097 /$ TP.0000000000001769

\section{SUMMARY OF RECOMMENDATION STATEMENTS}

All recommendation statements are not graded unless specified otherwise.

\section{CHAPTER 1: GOALS OF EVALUATION, FRAMEWORK FOR DECISION-MAKING, AND ROLES AND RESPONSIBILITIES}

\section{Goals and Principles of Evaluation}

1.1: The donor candidate's willingness to donate a kidney voluntarily without undue pressure should be verified.

1.2: The benefits and risks of kidney donation should be assessed for each donor candidate.

1.3: The decision to accept or exclude a donor candidate should follow transplant program policies.

1.4: Donor candidate decision-making should be facilitated through education and counseling on individualized risks and benefits, methods to minimize risks, and the need for postdonation follow-up.

1.5: For an accepted donor candidate, a plan for donation care and follow-up should be formulated to minimize risks of donation.

1.6: For an excluded donor candidate, a plan for any needed care and support should be formulated.

\section{Framework for Decision-Making}

1.7: The donor candidate, the intended recipient, and the transplant program must all agree with the decision to proceed with donation in concordance with transplant program policies and informed consent.

1.8: Transplant program policies must be defensible based on current understanding of the risks and benefits of kidney donation, and should apply to all donor candidates evaluated at the center.

1.9: Each transplant program should establish policies describing psychosocial criteria that are acceptable for donation, including any program constraints on acceptable relationships between the donor candidate and the intended recipient.

1.10: All donor candidates should be evaluated using the same criteria, regardless of whether donation is directed towards a designated recipient.

1.11: Each transplant program should establish policies describing medical criteria that are acceptable for donation, addressing when possible, numeric thresholds for short-term and long-term postdonation risks above which the transplant program will not proceed with donation. Risks should be expressed as absolute rather than relative risks.

1.12: When possible, transplant programs should provide each donor candidate with individualized quantitative estimates of short-term and long-term risks from donation, including recognition of associated uncertainty, in a manner that is easily understood by donor candidates.

1.13: Transplant programs should evaluate donor candidate risks in comparison to predetermined thresholds for acceptance. If a donor candidate's postdonation risk is above the transplant program's acceptable risk threshold, the risk is not acceptable for donation. If a donor candidate's postdonation risk is below the transplant program's acceptance threshold, the candidate makes the decision whether or not to proceed with donation.

1.14: If a donor candidate is not acceptable, the transplant program should explain the reason for nonacceptance to the donor candidate.

1.15: Transplant programs should protect donor candidate's privacy regarding the evaluation, including all considerations in the decision to donate or not.

\section{Roles and Responsibilities}

1.16: A multidisciplinary transplant program team knowledgeable in kidney donation and transplantation should evaluate, care for, and formulate a plan for donor care including long-term follow-up.

1.17: Transplant programs should minimize conflict of interest by providing at least one key team member not involved in the care or evaluation of the intended recipient who evaluates the donor candidate and participates in the determination of donor acceptance.

1.18: Transplant programs should conduct as efficient a donor evaluation as possible, meeting the needs of donor candidates, intended recipients and transplant programs.

\section{CHAPTER 2: INFORMED CONSENT}

\section{Process of Informed Consent}

2.1: Informed consent for donation should be obtained from the donor candidate in the absence of the intended recipient, family members and other persons who could influence the donation decision.

\section{Capacity for Decision Making}

2.2: The donor candidate's capacity to provide informed consent (ie, ability to understand the risks, benefits and consequences of donation) should be confirmed before proceeding with evaluation and donation.

2.3: Substitute decision makers should not be used on behalf of a donor candidate who lacks the capacity to provide informed consent (eg, children or those who are mentally challenged), except under extraordinary circumstances and only after ethical and legal review.

\section{Content of Disclosure}

2.4: Protocols should be followed to provide each donor candidate with information on:

- The processes of evaluation, donor acceptance, and follow-up

- The types of information that may be discovered during the evaluation, and what the transplant program will do with such information

- Individualized risks, benefits and expected outcomes of the donor evaluation, donation, and postdonation health, in cluding a discussion of the uncertainty in some outcomes 
- Treatment alternatives available to transplant candidates, and average expected outcomes

- How personal health information will be handled

- Availability of transplant program personnel for support
4.2: Donor candidates who smoke should be advised to quit at least 4 weeks before donation to reduce their risk of perioperative complications, and commit to lifelong abstinence to prevent long-term complications.

\section{Comprehension of Disclosed Information}

2.5: The donor candidate's understanding of the relevant information on the risks and benefits of donation should be confirmed before proceeding with donation.

\section{Voluntarism}

2.6: Donor candidates should have adequate time to consider information relevant to deciding whether they wish to donate or not.

2.7: A donor candidate's decision to withdraw at any stage of the evaluation process should be respected and supported in a manner that protects confidentiality.

2.8: A donor candidate who decides not to donate and has difficulty communicating that decision to the intended recipient should be assisted with this communication by the transplant program.

\section{CHAPTER 3: COMPATIBILITY TESTING, INCOMPATIBLE TRANSPLANTATION, AND PAIRED DONATION}

\section{Evaluation}

3.1: Donor ABO blood typing should be performed twice before donation to reduce the risk of unintended blood type incompatible transplantation.

3.2: Donor blood group A subtype testing should be performed when donation is planned to recipients with anti-A antibodies.

3.3: Human leukocyte antigen (HLA) typing for major histocompatibility complex (MHC) Class I (A, B, C) and Class II (DP, DQ, DR) should be performed in donor candidates and their intended recipients, and donor-specific antiHLA antibodies should be assessed in intended recipients.

\section{Counseling}

3.4: Donor candidates who are ABO blood group or HLA incompatible with their intended recipient should be informed of availability, risks, and benefits of treatment options, including kidney paired donation and incompatibility management strategies.

3.5: If a donor candidate and their intended recipient are blood type or crossmatch incompatible, transplantation should be performed only with an effective incompatibility management strategy.

3.6: Nondirected donor candidates should be informed of availability, risks and benefits of participating in kidney paired donation.

\section{CHAPTER 4: PREOPERATIVE EVALUATION AND MANAGEMENT}

4.1: Donor candidates should receive guideline-based evaluation and management used for other noncardiac surgeries to minimize risks of perioperative complications, including a detailed history and examination to assess risks for cardiac, pulmonary, bleeding, anesthesia-related and other perioperative complications.

\section{CHAPTER 5: PREDONATION KIDNEY FUNCTION}

\section{Evaluation}

5.1: Donor kidney function should be expressed as glomerular filtration rate (GFR) and not as serum creatinine concentration.

5.2: Donor GFR should be expressed in $\mathrm{mL} / \mathrm{min}$ per $1.73 \mathrm{~m}^{2}$ rather than $\mathrm{mL} / \mathrm{min}$.

5.3: Donor glomerular filtration rate (GFR) should be estimated from serum creatinine $\left(\mathrm{eGFR}_{\mathrm{cr}}\right)$ for initial assessment, following recommendations from the KDIGO 2012 CKD guideline.

5.4: Donor GFR should be confirmed using one or more of the following measurements, depending on availability:

- Measured GFR (mGFR) using an exogenous filtration marker, preferably urinary or plasma clearance of inulin, urinary or plasma clearance of iothalamate, urinary or plasma clearance of ${ }^{51} \mathrm{Cr}$-EDTA, urinary or plasma clearance of iohexol, or urinary clearance of 99m Tc-DTPA

- Measured creatinine clearance $(\mathrm{mCrCl})$

- Estimated GFR from the combination of serum creatinine and cystatin $\mathrm{C}\left(\mathrm{eGFR}_{\text {cr-cys }}\right)$ following recommendations from the KDIGO 2012 CKD guideline

- Repeat estimated GFR from serum creatinine $\left(\mathrm{eGFR}_{\mathrm{cr}}\right)$

5.5: If there are parenchymal, vascular or urological abnormalities or asymmetry of kidney size on renal imaging, single kidney GFR should be assessed using radionuclides or contrast agents that are excreted by glomerular filtration (eg, $\left.{ }^{99 \mathrm{~m}} \mathrm{Tc}-\mathrm{DTPA}\right)$.

\section{Selection}

5.6: GFR of $90 \mathrm{~mL} / \mathrm{min}$ per $1.73 \mathrm{~m}^{2}$ or greater should be considered an acceptable level of kidney function for donation.

5.7: The decision to approve donor candidates with GFR 60 to $89 \mathrm{~mL} / \mathrm{min}$ per $1.73 \mathrm{~m}^{2}$ should be individualized based on demographic and health profile in relation to the transplant program's acceptable risk threshold.

5.8: Donor candidates with GFR less than $60 \mathrm{~mL} / \mathrm{min}$ per $1.73 \mathrm{~m}^{2}$ should not donate.

5.9: When asymmetry in GFR, parenchymal abnormalities, vascular abnormalities, or urological abnormalities are present but do not preclude donation, the more severely affected kidney should be used for donation.

\section{Counseling}

5.10: We suggest that donor candidates be informed that the future risk of developing kidney failure necessitating treatment with dialysis or transplantation is slightly higher because of donation; however, average absolute risk in the 15 years following donation remains low. (2C)

\section{CHAPTER 6: PREDONATION ALBUMINURIA}

6.1: Donor proteinuria should be measured as albuminuria, not total urine protein.

\section{Evaluation}


6.2: Initial evaluation of donor albuminuria (screening) should be performed using urine albumin-to-creatinine ratio (ACR) in a random (untimed) urine specimen.

6.3: Donor albuminuria should be confirmed using:

- Albumin excretion rate (AER, $\mathrm{mg} / \mathrm{day}[\mathrm{mg} / \mathrm{d}])$ in a timed urine specimen

- Repeat ACR if AER cannot be obtained

\section{Selection}

6.4: Urine AER less than $30 \mathrm{mg} / \mathrm{d}$ should be considered an acceptable level for donation.

6.5: The decision to approve donor candidates with AER 30 to $100 \mathrm{mg} / \mathrm{d}$ should be individualized based on demographic and health profile in relation to the transplant program's acceptable risk threshold.

6.6: Donor candidates with urine AER greater than $100 \mathrm{mg} / \mathrm{d}$ should not donate.

\section{CHAPTER 7: PREDONATION HEMATURIA}

\section{Evaluation}

7.1: Donor candidates should be assessed for microscopic hematuria.

7.2: Donor candidates with persistent microscopic hematuria should undergo testing to identify possible causes, which may include:

- Urinalysis and urine culture to assess for infection

- Cystoscopy and imaging to assess for urinary tract malignancy

- 24-hour urine stone panel to assess for nephrolithiasis and/or microlithiasis

- Kidney biopsy to assess for glomerular disease (eg, thin basement membrane nephropathy, IgA nephropathy, Alport syndrome)

\section{Selection}

7.3: Donor candidates with hematuria from a reversible cause that resolves (eg, a treated infection) may be acceptable for donation.

7.4: Donor candidates with IgA nephropathy should not donate.

\section{CHAPTER 8: KIDNEY STONES}

\section{Evaluation}

8.1: Donor candidates should be asked about prior kidney stones, and related medical records should be reviewed if available.

8.2: The imaging performed to assess anatomy before donor nephrectomy (eg, computed tomography angiogram) should be reviewed for the presence of kidney stones.

8.3: Donor candidates with prior or current kidney stones should be assessed for an underlying cause.

\section{Selection}

8.4: The acceptance of a donor candidate with prior or current kidney stones should be based on an assessment of stone recurrence risk and knowledge of the possible consequences of kidney stones after donation.

\section{Counseling}

8.5: Donor candidates and donors with current or prior kidney stones should follow general population, evidencebased guidelines for the prevention of recurrent stones.

\section{CHAPTER 9: HYPERURICEMIA, GOUT, AND MINERAL AND BONE DISEASE}

\section{Evaluation}

9.1: Donor candidates should be asked about prior episodes of gout.

\section{Counseling}

9.2: Donor candidates may be informed that donation is associated with an increase in serum uric acid concentration, which may increase the risk for gout.

9.3: Donor candidates and donors with prior episodes of gout should be informed of recommended methods to reduce their risk of future episodes of gout.

\section{CHAPTER 10: PREDONATION BLOOD PRESSURE}

\section{Evaluation}

10.1: Blood pressure should be measured before donation on at least 2 occasions by clinical staff trained in accurate measurement technique, using equipment calibrated for accuracy.

10.2: When the presence or absence of hypertension in a donor candidate is indeterminate based on history and clinic measurements (eg, blood pressure is high normal or variable), blood pressure should be further evaluated using ambulatory blood pressure monitoring (ABPM) or repeated using standardized blood pressure measurements.

\section{Selection}

10.3: Normal blood pressure, as defined by guidelines for the general population in the country or region where donation is planned, is acceptable for donation.

10.4: Donor candidates with hypertension that can be controlled to systolic blood pressure less than $140 \mathrm{~mm} \mathrm{Hg}$ and diastolic blood pressure less than $90 \mathrm{~mm} \mathrm{Hg}$ using 1 or 2 antihypertensive agents, who do not have evidence of target organ damage, may be acceptable for donation. The decision to approve donor candidates with hypertension should be individualized based on demographic and health profile in relation to the transplant program's acceptable risk threshold.

\section{Counseling}

10.5: Donor candidates should be counseled on lifestyle interventions to address modifiable risk factors for hypertension and cardiovascular disease, including healthy diet, smoking abstinence, achievement of healthy body weight, and regular exercise according to guidelines for the general population. These measures should be initiated before donation and maintained lifelong.

10.6: We suggest that donor candidates should be informed that blood pressure may rise with aging, and that donation may accelerate a rise in blood pressure and need for antihypertensive treatment over expectations with normal aging. (2D)

\section{CHAPTER 11: PREDONATION METABOLIC AND LIFESTYLE RISK FACTORS}

\section{Identification of Metabolic and Lifestyle Risk Factors}

11.1: Risk factors for kidney and cardiovascular disease should be identified before donation and addressed by counseling to promote long-term health. 


\section{Obesity}

11.2: Body mass index (BMI) should be computed based on weight and height measured before donation, and classified based on World Health Organization (WHO) criteria for the general population or race-specific categories.

11.3: The decision to approve donor candidates with obesity and $\mathrm{BMI}>30 \mathrm{~kg} / \mathrm{m}^{2}$ should be individualized based on demographic and health profile in relation to the transplant program's acceptable risk threshold.

11.4: Donor candidates who have had bariatric surgery should be assessed for risk of nephrolithiasis.

\section{Glucose Intolerance}

11.5: Donor candidates should be asked about prior diagnosis of diabetes mellitus, gestational diabetes, and family history of diabetes.

11.6: Glycemia should be assessed by fasting blood glucose and/or glycated hemoglobin $\left(\mathrm{HbA}_{1 \mathrm{c}}\right)$ before donation.

11.7: 2-hour glucose tolerance or $\mathrm{HbA}_{1 \mathrm{c}}$ testing should be performed in donor candidates with elevated fasting blood glucose, history of gestational diabetes, or family history of diabetes in a first-degree relative, and results should be used to classify diabetes or prediabetes status using established criteria for the general population.

11.8: Donor candidates with type 1 diabetes mellitus should not donate.

11.9: The decision to approve donor candidates with prediabetes or type 2 diabetes should be individualized based on demographic and health profile in relation to the transplant program's acceptable risk threshold.

11.10: Donor candidates with prediabetes or type 2 diabetes should be counseled that their condition may progress over time and may lead to end-organ complications.

\section{Dyslipidemias}

11.11: Fasting lipid profile (including total cholesterol, LDL-C, HDL-C and triglycerides) should be measured as part of an overall cardiovascular risk assessment before donation.

11.12: The decision to approve donor candidates with dyslipidemia should be individualized based on demographic and health profile in relation to the transplant program's acceptable risk threshold.

\section{Tobacco Use}

11.13: The use of tobacco products should be assessed before donation.

11.14: Donor candidates who use tobacco products should be counseled on the risks of perioperative complications, cancer, cardio-pulmonary disease and kidney failure, should be advised to abstain from use of tobacco products, and should be referred to a tobacco cessation support program if possible.

11.15: The decision to approve donor candidates who are active tobacco users should be individualized based on demographic and health profile in relation to the transplant program's acceptable risk threshold.

\section{CHAPTER 12: PREVENTING INFECTION TRANSMISSION}

\section{Evaluation}

12.1: Risk for human immunodeficiency virus (HIV), hepatitis $B$ virus (HBV), and hepatitis $\mathrm{C}$ virus (HCV) infections should be assessed before donation.
12.2: Donor candidates should be assessed for factors associated with an increased likelihood of endemic or unexpected infections, including geographic, seasonal, occupational, animal and environmental exposures.

12.3: Donor candidates should complete a urinalysis and testing for HIV, HBV, HCV, cytomegalovirus (CMV), EpsteinBarr virus (EBV), and Treponema pallidum (syphilis).

12.4: If indicated by regional epidemiology or individual history, donor candidates should complete testing for Mycobacterium tuberculosis, Strongyloides, Trypanosoma cruzi, West Nile virus, Histoplasmosis, and/or Coccidiomycosis.

12.5: Transplant programs should develop protocols to screen donor candidates for emerging infections in consultation with local public health specialists.

12.6: In general, donor infection risk factor and microbiological assessments should be performed or updated as close in time to donation as possible. For HIV, HBV and HCV, screening should be current within 28 days of donation.

\section{Selection}

12.7: If a donor candidate is found to have a potentially transmissible infection, then the donor candidate, intended recipient and transplant program team should weigh the risks and benefits of proceeding with donation.

\section{CHAPTER 13: CANCER SCREENING}

\section{Evaluation}

13.1: Donor candidates should undergo cancer screening consistent with clinical practice guidelines for the country or region where the donor candidate resides. Transplant programs should ensure that screening is current according to guideline criteria at the time of donation.

\section{Selection}

13.2: In general, donor candidates with active malignancy should be excluded from donation. In some cases of active malignancy with low transmission risk, a clear management plan and minimal risk to the donor, donation may be considered.

13.3: A kidney with a small simple (Bosniak I) cyst can be left in the donor, particularly if there are compelling reasons for donating the contralateral kidney.

13.4: Donation of a kidney with a Bosniak II renal cyst should proceed only after assessment for the presence of solid components, septations, and calcifications on the preoperative computed tomography scan (or magnetic resonance imaging) to avoid accidental transplantation of a kidney with cystic renal cell carcinoma.

13.5: Donor candidates with high grade Bosniak renal cysts (III or higher) or small (T1a) renal cell carcinoma curable by nephrectomy may be acceptable for donation on a caseby-case basis.

13.6: Donor candidates with a history of treated cancer that has a low risk of transmission or recurrence may be acceptable for donation on a case-by-case basis.

\section{CHAPTER 14: EVALUATION OF GENETIC KIDNEY DISEASE}

\section{Evaluation}

14.1: Donor candidates should be asked about their family history of kidney disease, and when present, the type 
of disease, time of onset, and extra-renal manifestations associated with the disease.

14.2: When the intended recipient is genetically related to the donor candidate, the cause of the intended recipient's kidney failure should be determined whenever possible. The intended recipient should consent to share this medical information with the donor evaluation team, and with the donor candidate if it could affect the decision to donate.

\section{Selection}

14.3: Donor candidates found to have a genetic kidney disease that can cause kidney failure should not donate.

\section{Counseling}

14.4: Donor candidates must provide informed consent for genetic testing if indicated as part of their evaluation. Donor candidates should be informed of the possible effects of receiving a diagnosis of a genetic kidney disease, such as any impact on their ability to obtain health or life insurance.

14.5: In cases where it remains uncertain whether the donor candidate has a genetic kidney disease and whether the disease can cause kidney failure, donation should proceed only after informing the donor candidate of the risks of donation if the disease manifests later in life.

\section{Autosomal Dominant Polycystic Kidney Disease (ADPKD)}

14.6: Donor candidates with ADPKD should not donate.

14.7: Donor candidates with a family history of ADPKD in a first-degree relative may be acceptable for donation if they meet age-specific imaging or genetic testing criteria that reliably exclude ADPKD.

\section{Apolipoprotein L1 (APOL1) Risk Alleles}

14.8: Apolipoprotein L1 (APOL1) genotyping may be offered to donor candidates with sub-Saharan African ancestors. Donor candidates should be informed that having 2 APOL 1 risk alleles increases the lifetime risk of kidney failure but that the precise kidney failure risk for an affected individual after donation cannot currently be quantified.

\section{CHAPTER 15: PREGNANCY}

\section{Evaluation}

15.1: Female donor candidates should be asked about future childbearing plans.

15.2: Female donor candidates should be asked about prior hypertensive disorders of pregnancy (eg, gestational hypertension, preeclampsia, or eclampsia).

15.3: Local guidelines should be followed to confirm the absence of pregnancy before performing radiologic tests, including abdominal computed tomography (with iodinated contrast) or nuclear medicine GFR testing.

\section{Selection}

15.4: Women should not donate while pregnant.

15.5: Women should not be excluded from donation solely because they desire to conceive children after donation.

15.6: Women with a prior hypertensive disorder of pregnancy may be acceptable for donation if their longterm postdonation risks are acceptable.

15.7: A decision to proceed with donation in the year after childbirth should consider the psychological needs of mother and child, and should include anesthesia and analgesia planning for nursing mothers.

\section{Counseling}

15.8: Women with childbearing potential should be informed of the need to avoid becoming pregnant from the time of approval for donation to the time of recovery after nephrectomy; a quantitative human chorionic gonadotropin ( $\beta-h C G)$ pregnancy test should be performed and confirmed as negative immediately before donation.

15.9: We suggest that women with childbearing potential be counseled about the effects donation may have on future pregnancies, including the possibility of a greater likelihood of being diagnosed with gestational hypertension or preeclampsia. $(2 \mathrm{C})$

15.10: Women with a prior hypertensive disorder of pregnancy should be informed about their long-term risks.

15.11: Women with childbearing potential who proceed with donation should be counseled on how to reduce the risk of complications in future pregnancies.

\section{CHAPTER 16: PSYCHOSOCIAL EVALUATION}

\section{Evaluation}

16.1: Donor candidates should receive in-person psychosocial evaluation, education and planning from health professionals experienced in the psychosocial concerns of donor candidates and donors.

16.2: To ensure voluntariness, at least a portion of the psychosocial evaluation of the donor candidate should be performed in the absence of the intended recipient, family members and other persons who could influence the donation decision.

16.3: Whenever possible, the psychosocial evaluation of the donor candidate should be performed by health professionals not involved in the care of the intended recipient.

16.4: Transplant programs should follow protocols for assessing the donor candidate's psychosocial suitability, available support, preparation and concerns for donation.

\section{Selection}

16.5: Transplant programs should follow protocols defining psychosocial factors that either exclude donation, or prevent further evaluation until resolution.

\section{Disclosures and Support}

16.6: We suggest that donor candidates be informed that donors usually have good quality of life after donation (2D).

16.7: Transplant programs should assist donor candidates and donors in receiving psychosocial or psychiatric support as needed.

\section{CHAPTER 17: ACCEPTABLE SURGICAL APPROACHES FOR DONOR NEPHRECTOMY}

17.1: Renal imaging (eg, computed tomographic angiography) should be performed in all donor candidates to assess renal anatomy before nephrectomy.

17.2: The surgeon should have adequate training and experience for the surgical approach used for the donor nephrectomy.

17.3: We suggest that "mini-open" laparoscopy or handassisted laparoscopy by trained surgeons should be offered as optimal approaches to donor nephrectomy. 
However, in some circumstances, such as for donors with extensive previous surgery and/or adhesions, and at centers where laparoscopy is not routinely performed, open nephrectomy (flank or laparotomy) may be acceptable. $(2 D)$

17.4: Robotic, single-port, and natural orifice transluminal nephrectomy should generally not be used for donor nephrectomy.

17.5: Nontransfixing clips, (eg, Weck Hem-o-lok) should not be used to ligate the renal artery in donor nephrectomy; instead, renal artery transfixation by suture ligature or anchor staple within the vessel wall should be used.

17.6: In the absence of reasons to procure the right kidney (vascular, urological or other abnormalities), the left kidney should be procured in laparoscopic donor nephrectomy because of the relative technical ease associated with a longer venous pedicle.

17.7: We suggest laparoscopic procurement of the right rather than the left living donor kidney may be performed if the surgeon has adequate training and experience. $(2 D)$

17.8: Procurement of a living donor kidney with 3 or more arteries should only be undertaken by surgeons with adequate experience.

17.9: A donor candidate with atherosclerotic renal artery disease or fibromuscular dysplasia involving the orifices of both renal arteries should not donate.

\section{CHAPTER 18: ETHICAL, LEGAL AND POLICY CONSIDERATIONS}

\section{Ethical and Legal Framework}

18.1: Local laws and regulations on living donation should be followed and explained as needed to donor candidates.

18.2: Where local laws or policies impede the ethical practice of living donation, avenues to advocate for change should be explored.

18.3: Autonomy (self-determination) in the willingness or not to be considered as a living donor should be respected during all phases of the evaluation and donation processes. Transplant programs should support autonomy through a fully informed consent process.

\section{Policies for Donor Candidate Identification}

18.4: Public awareness of opportunities for living donation should be increased through education, donor advocacy, evaluation efficiencies, and removal of disincentives.

18.5: Transplant candidates should be assisted in identifying living donor candidates, as long as these efforts respect donor autonomy and do not exert undue pressure to donate.

18.6: Donor candidates should be informed of the dangers of transplant tourism.

18.7: Transplant programs should define and disclose their policies for the acceptance of donor candidates identified through public solicitation.

\section{Financial Support}

18.8: Donor candidates should be informed of the availability of legitimate financial assistance for expenses from evaluation and donation.

\section{Communication of Policies}

18.9: Nondirected donors and donors participating in paired donation should be informed of the transplant program's policy on contact with the recipient and other paired donation participants at all stages in the donation process.

18.10: Transplant programs should disclose the extent of the expected postdonation program-patient relationship before donation, including whether the donor can seek medical care at the transplant center after donation.

18.11: Regional policies should ensure access to kidney replacement therapy (dialysis and/or transplantation) for donors who develop kidney failure.

\section{CHAPTER 19: POSTDONATION FOLLOW-UP CARE}

19.1: A personalized postdonation care plan should be provided before donation to clearly describe follow-up care recommendations, who will provide the care, and how often.

19.2: The following should be performed at least annually postdonation:

- Blood pressure measurement

- BMI measurement

- Serum creatinine measurement with GFR estimation

- Albuminuria measurement

- Review and promotion of a healthy lifestyle including regular exercise, healthy diet and abstinence from tobacco

- Review and support of psychosocial health and well-being

19.3: Donors should be monitored for CKD, and those meeting criteria for CKD should be managed according to the 2012 KDIGO CKD Guideline.

19.4: Donors should receive age-appropriate healthcare maintenance, and management of clinical conditions and health risk factors according to clinical practice guidelines for the regional population.

\section{METHODS FOR GUIDELINE DEVELOPMENT}

\section{AIM}

The overall aim of this project was to develop an evidencebased clinical practice guideline on the evaluation and care of living kidney donors. The guideline consists of recommendation statements and supporting rationale, including summaries of systematically generated evidence on relevant predefined clinical topics. The general Kidney Disease: Improving Global Outcomes (KDIGO) guideline development method is described at: http://www.kdigo.org/home/guidelines/development, and unique considerations for application to the current guideline are discussed below.

\section{PROCESS}

The development of the KDIGO Clinical Practice Guideline on the Evaluation and Care of Living Kidney Donors included:

- Appointing work group (WG) members and the Evidence Review Team (ERT)

- Discussing guideline development processes and methods

- Developing guideline scope of work, including submission of proposed topics for public comment, and refining topics based on public feedback

- Identifying populations, interventions, risk factors, and outcomes of interest

- Selecting topics for systematic evidence review and conducting the literature review by: 
- Developing and implementing literature search strategies

- Screening abstracts and retrieving full-text articles based on predefined eligibility criteria

- Creating data extraction forms

- Extracting data from individual studies

- Standardizing methodology for evidence quality assessment

- Tabulating data from individual studies into summary tables and performing critical appraisal of the literature

- Grading quality of evidence for each outcome across studies, and assessing the overall quality of evidence across outcomes with the aid of evidence profiles

- Where applicable, grading recommendation strength based on the quality of evidence and other considerations for recommendations with available evidence in the systematic review

- Using de novo evidence generation, WG consensus and prior KDIGO Guidelines to develop recommendations on topics not assigned to formal systematic review, but deemed important for the guideline

- Developing supporting rationale and finalizing guideline recommendations

- Distributing the guideline draft for public review from November to December 2015

- Editing the guideline based on public feedback, and creating the point-by-point response

The WG cochairs, KDIGO cochairs and ERT met to review the guideline development process, evidence review topics, and systematic review process. After this, 2 inperson meetings were held May 2014 and September 2014 with the WG, ERT, KDIGO cochairs and KDIGO support staff to review the available evidence, formulate recommendation statements and their rationale. Details of each chapter were discussed by teleconference between the WG cochairs, WG members and support staff before each in-person meeting, and by email. The WG cochairs, ERT, and support staff also held regular calls until completion of the evidence review and systematic review report, and the WG cochairs, KDIGO Chair and support staff met by regular teleconferences until guideline completion.

\section{Commissioning of the WG and the ERT}

The KDIGO cochairs appointed the WG cochairs, who then assembled the WG to include individuals with expertise in nephrology, organ donation, transplantation, surgery, bioethics, psychology, epidemiology and public policy. The University of Minnesota Veterans Administration Center for Chronic Disease Outcomes Research in Minneapolis, Minnesota was contracted to conduct systematic evidence review and provided expertise in guideline development methodology. The ERT was led by guideline methodologists and clinicians with expertise in nephrology and evidence-based clinical practice guideline development.

\section{Defining the Scope and Topics}

The WG cochairs and the ERT defined the scope and goals of the guideline (including a list of critical and important outcomes) and drafted a preliminary list of topics and key clinical questions. The project scope was submitted for public comment in October 2013 and refined based on the feedback. The WG and ERT further developed and refined each topic and specified screening criteria, literature search strategies, and data extraction forms.

\section{Establishing the Process for Guideline Development}

The ERT performed a systematic literature search and organized abstract and article screening. The ERT also coordinated the methodological and analytical processes and defined and standardized the methodology for performing literature searches, data extraction, and summarizing the evidence. The WG wrote the recommendation statements and supporting rationale, in consultation with the ERT. For recommendations with available evidence in the systematic review, the ERT reviewed draft recommendation statements and their corresponding grades for consistency with the conclusions of the evidence systematic review.

\section{Formulating Questions of Interest}

The KDIGO WG developed a scoping document to describe topics to be covered by the guideline. To inform the WG's initial work, the ERT searched for and identified existing clinical practice guidelines related to living kidney donation. The ERT extracted recommendations from prior guidelines on the identified topics of interest for the current guideline and provided a summary table of this information. This document was distributed to the WG in 2013. Certain topics within the scoping document were considered relevant to the systematic review based on importance and feasibility, and the ERT developed key research questions to address these topics (Table 1). The Population, Intervention, Comparator, Outcome, study Design, and Duration of follow-up (PICODDs) criteria for the systematic review (Table 2) are described in detail in an accompanying publication. ${ }^{3}$ Outcomes were selected and ranked by assessing patient-centeredness. Risk assessment periods were defined as perinephrectomy/ postnephrectomy (within 90 days of donation), and longerterm (after 90 days postdonation until the end of available follow-up). Long-term outcomes were based on a mean follow-up of at least 5 years.

\section{Ranking of Outcomes}

The WG ranked outcomes of interest based on their importance for informing clinical decision making, as "critical",

\section{TABLE 1.}

\section{Key questions defining the evidence review}

Key Question 1: What is the incidence of perinephrectomy/postnephrectomy outcomes among living kidney donors undergoing different types of nephrectomy procedures?

Key Question 2: Does the incidence of perinephrectomy/postnephrectomy outcomes vary by demographic subgroup (age, race, sex)?

Key Question 3: Does the incidence of perinephrectomy/postnephrectomy outcomes vary by the presence of donor IMAs (ie, obesity, hypertension, glucose intolerance)?

Key Question 4: What is the incidence of long-term health outcomes for living kidney donors compared with healthy nondonors?

Key Question 5: Does the incidence of long-term living kidney donor outcomes vary by demographic subgroup (age, race, sex)?

Key Question 6: Does the incidence of long-term living kidney donor outcomes vary by the presence of donor IMAs (ie, obesity, hypertension, glucose intolerance)?

Key Question 7: What is the incidence of maternal and fetal outcomes among female living kidney donors who become pregnant after donation compared with healthy nondonors?

IMA, isolated medical abnormality. 
TABLE 2.

Systematic review screening criteria

Living kidney donor outcomes

\section{Perinephrectomy/postnephrectomy}

Population Living kidney donors

Intervention

Nephrectomy performed post-1994

Nephrectomy in donors with IMAs

Comparator Nephrectomy with different types of surgery; Nephrectomy in donors without IMAs

Outcomes

Study design

Critical: all-cause mortality

High Importance: CVD event

Moderate Importance: perinephrectomy/postnephrectomy

complications; time to return to usual activities

Intermediate Outcomes: blood loss; length

of hospital stay

Systematic reviews, randomized controlled

trials, and observational studies

Duration of follow-up Systematic reviews with outcomes measured up to 90 d postnephrectomy were extracted

Full-text screening identified studies with outcomes measured up to 1 y postdonation for extraction
Long-term

Living kidney donors

Living kidney donors — demographic subgroup (age, sex, race)

Living kidney donors with specific IMAs

Donors related to recipient or with known family history of kidney disease

Women with postdonation pregnancy

Living kidney donation

Healthy nondonors ${ }^{a}$ (ie, nondonor with medical characteristics suggesting they meet living donor criteria))

Living kidney donors-demographic subgroups (age, sex, race)

Healthy nondonors ${ }^{2}$ - demographic subgroups (age, sex, race)

Living kidney donors - without specific IMAs

Healthy nondonors ${ }^{\text {a }}$ - with specific IMAs

Donors with unrelated recipient and no family history of kidney disease

Donors with predonation pregnancy

Healthy nondonors with pregnancy

Critical: all-cause mortality, CVD event, kidney failure, fetal death

High importance: psychosocial outcomes, major pregnancy complications

Moderate importance: fragility fractures, gastrointestinal bleeding,

kidney stones, minor pregnancy complications

Intermediate outcomes: kidney function, proteinuria, hypertension

Systematic reviews, randomized controlled trials, and observational studies

Full-text screening identified studies with total sample sizes (donor and comparator combined) more than 50; studies with

total sample sizes more than 100 were extracted

Full-text screening identified studies with outcomes measured 1 y or later postdonation; studies with a mean duration of $5 \mathrm{y}$ or more postdonation were extracted

\footnotetext{
a "Healthy" nondonor comparison groups must have matched or controlled for demographic and health characteristics. CVD, cardiovascular disease; IMA, isolated medical abnormality.
}

"high" or "moderate." The hierarchy of perinephrectomy/ postnephrectomy and long-term outcomes is noted in Table 2. "Intermediate outcomes" are defined as events on the pathway to clinical outcomes of interest.

\section{Literature Searches and Article Selection}

The ERT searched Ovid Medline, Ovid EMBASE, and the Cochrane Library to identify previous systematic reviews, randomized controlled trials (RCTs), and observational studies published and indexed in bibliographic databases through September 2014. The ERT search strategy included relevant medical subject headings and natural language terms for the concept of living kidney donation (Supplemental Appendix A, SDC, http://links.lww.com/TP/B432). These terms were combined with filters to select RCTs, systematic reviews, and observational studies. Bibliographic database searches were supplemented with citation searches of highly relevant systematic reviews.

Two independent investigators reviewed titles and abstracts of search results published after 2003 to identify systematic reviews, trials and observational studies relevant to the key questions. The ERT relied on citation searching of relevant systematic reviews to identify relevant studies published before 2004. Citations deemed eligible by either investigator underwent full text screening. Two investigators independently screened full text to determine if PICODD criteria were met. A third investigator resolved discrepancies. The ERT documented the inclusion and exclusion status of citations undergoing full-text screening. The ERT often revisited the screening process as the WG identified new outcomes or subgroups not included in the original PICODDs. Screening criteria were liberal. The ERT did not extract data from all eligible studies. To capture the highest quality and most relevant and meaningful data as efficiently as possible, the ERT extracted data only from previous systematic reviews for perinephrectomy/ postnephrectomy outcomes Key Questions (KQ1-3) and from systematic reviews and select observational studies for long-term outcomes (KQ 4-7). The ERT extracted longterm outcomes data from observational studies with sample sizes over 100 and mean follow-up time of at least 5 years.

Explicit recognition of perspectives of comparison is critical for drawing inferences about donor health outcomes (eg, estimation of predonation risk, absolute postdonation risk, and donation-attributable risk) ${ }^{4}$ (Figure 1), and types of comparison were a critical consideration throughout the development of this guideline, including the design and conduct of the evidence review. Studies reporting long-term outcomes were required to include a nondonor comparison 


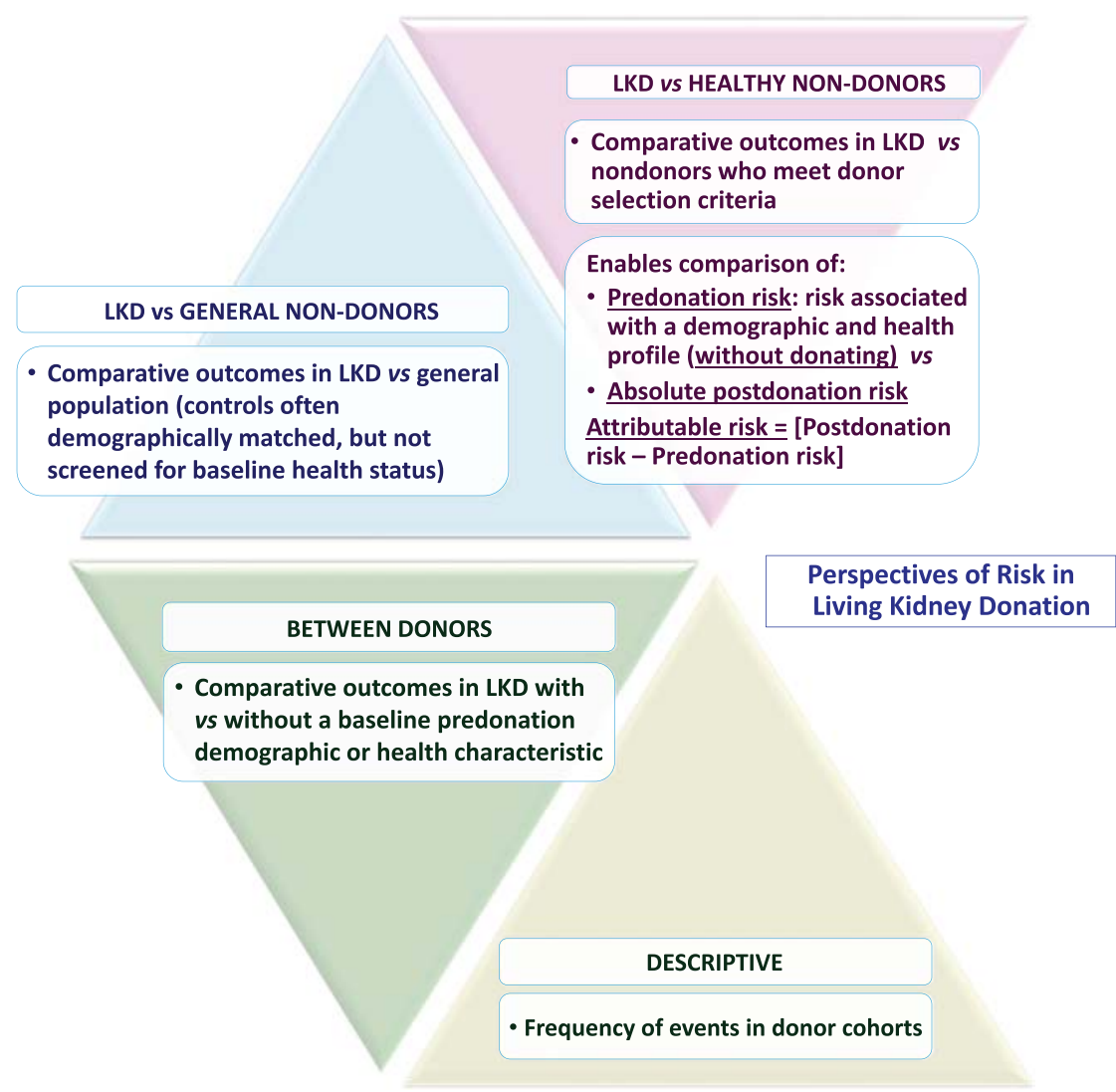

FIGURE 1. Perspectives of risk in living kidney donation. These perspectives provide a framework for assessment of donor outcomes, interpretation of observations, patient communication, and future research design. LKD, living kidney donors. Adapted with permission from Lentine KL, Segev DL. Understanding and communicating medical risks for living kidney donors: a matter of perspective. J Am Soc Nephrol. 2017;28:12-24. ${ }^{4}$

group with baseline health characteristics similar to kidney donors. Studies comparing living kidney donors to the general population were not eligible.

\section{Data Extraction and Summary Tables}

For perinephrectomy/postnephrectomy outcomes defined in the search criteria, the ERT extracted data from relevant comparisons in recent systematic reviews rather than performing a de novo extraction process. The ERT extracted relevant narrative information from systematic reviews that did not provide meta-analyses. For long-term outcomes defined in the search criteria, the ERT extracted data from observational studies and extracted pooled results from previous meta-analyses.

One investigator extracted relevant study, population demographic, and outcomes data from studies eligible for full extraction. Data fields extracted included author, year of publication, setting, donor and comparison populations, inclusion and exclusion criteria, donor and comparison characteristics, follow-up duration, descriptions, and results of outcomes. Relevant data were extracted into tables for descriptive analysis. In several cases, many comparisons were made within the same published study. In these cases, the ERT extracted relevant comparisons but did not extract ineligible comparisons.

\section{Assessment of Prior Systematic Review Quality and Individual Study Risk of Bias}

The ERT assessed the quality of eligible systematic reviews using modified A Measurement Tool to Assess Systematic
Reviews (AMSTAR) criteria. $^{5}$ The ERT assessed risk of bias for observational studies using an instrument developed based on the Research Triangle Institute Item Bank for assessing risk of bias and confounding in observational studies of interventions or exposures. ${ }^{6}$ In this instrument, overall summary risk of bias is based upon the collective risk of bias inherent in each domain and confidence that results are believable given study limitations. The ERT used overall summary risk of bias assessments when grading evidence quality as described below.

\section{Evidence Profiles}

The structured GRADE approach was used to grade the quality of the overall evidence (Table 3). Evidence profiles consisting of tables examining all relevant outcomes, including a summary of the results and judgments about the certainty and quality of the evidence, were used to facilitate this process. The GRADE approach is prescriptive in how evidence quality is assessed. The study design suggests the initial quality of evidence: high for RCTs and low for observational studies. Of note for the current guideline, most studies of outcomes in living kidney donation are not ethically or practically amenable to randomized controlled designs, limiting the possible quality rating based on the nature of possible study designs. Evidence quality is then lowered by one level if the studies in the evidence base for a comparison have serious risk of bias, and by 2 levels is the evidence base has very serious risk of bias. Evidence quality is also lowered when results across studies are inconsistent or 
TABLE 3.

Evidence quality assessment criteria

\begin{tabular}{|c|c|c|c|}
\hline Study design & Quality of evidence & Lower if & Higher if \\
\hline \multirow[t]{2}{*}{ Randomized trial } & High & $\begin{array}{l}\text { Risk of Bias } \\
\quad-1 \text { Serious } \\
-2 \text { Very serious }\end{array}$ & $\begin{array}{l}\text { Large effect } \\
\quad+1 \text { Large } \\
\quad+2 \text { Very Large }\end{array}$ \\
\hline & Moderate & $\begin{array}{l}\text { Inconsistency } \\
\qquad 1 \text { Serious } \\
-2 \text { Very serious }\end{array}$ & $\begin{array}{l}+1 \text { If all plausible confounding would reduce a demonstrated effect or } \\
+1 \text { If all plausible confounding would suggest a spurious } \\
\text { effect when results show no effect }\end{array}$ \\
\hline \multirow[t]{2}{*}{ Observational study } & Low & $\begin{array}{l}\text { Indirectness } \\
\quad-1 \text { Serious } \\
-2 \text { Very serious } \\
\text { Imprecision } \\
-1 \text { Serious } \\
-2 \text { Very serious }\end{array}$ & \\
\hline & Very Low & $\begin{array}{l}\text { Publication bias } \\
-1 \text { Likely }\end{array}$ & \\
\hline
\end{tabular}

very inconsistent, if the relationship between the intervention and the outcome is indirect, or if the outcome does not directly influence patient well-being. Additionally, evidence quality is downgraded when estimates are imprecise and publication bias is likely. Evidence quality improves with a large effect size. A large effect size includes a relative risk (RR) confidence interval (CI) lower limit of at least 2; a very large effect size includes a RR CI lower limit of 5. Evidence quality is also increased when an effect is demonstrated after all plausible confounding has been addressed. Complete evidence profiles for KQs 1-7 can be found in the Supplemental Evidence Report: Outcomes of Living Kidney Donation: A Systematic Review for a Clinical Practice Guideline by the Kidney Diseases Improving Global Outcomes (KDIGO), SDC, http://links.lww.com/TP/B434. Evidence on long-term outcomes as reviewed and appraised by the ERT has also been published separately as part of this guideline systematic review. ${ }^{3}$

\section{Results of the Systematic Review}

The ERT's search identified 4530 citations, of which 414 met criteria for full text review after title and abstract screening. The ERT identified an additional 70 references via supplemental citation searching, yielding a total of 484 references for full text review (Figure 2). Studies excluded after full-text review and exclusion reasons are listed in Supplemental Appendix B, SDC, http://links.lww.com/ TP/B432, of the evidence review publication.

The ERT extracted study characteristics, conducted systematic review quality assessments and risk-of-bias assessments, and extracted relevant outcomes into evidence tables for all studies eligible for extraction (Supplemental Appendix C, SDC,

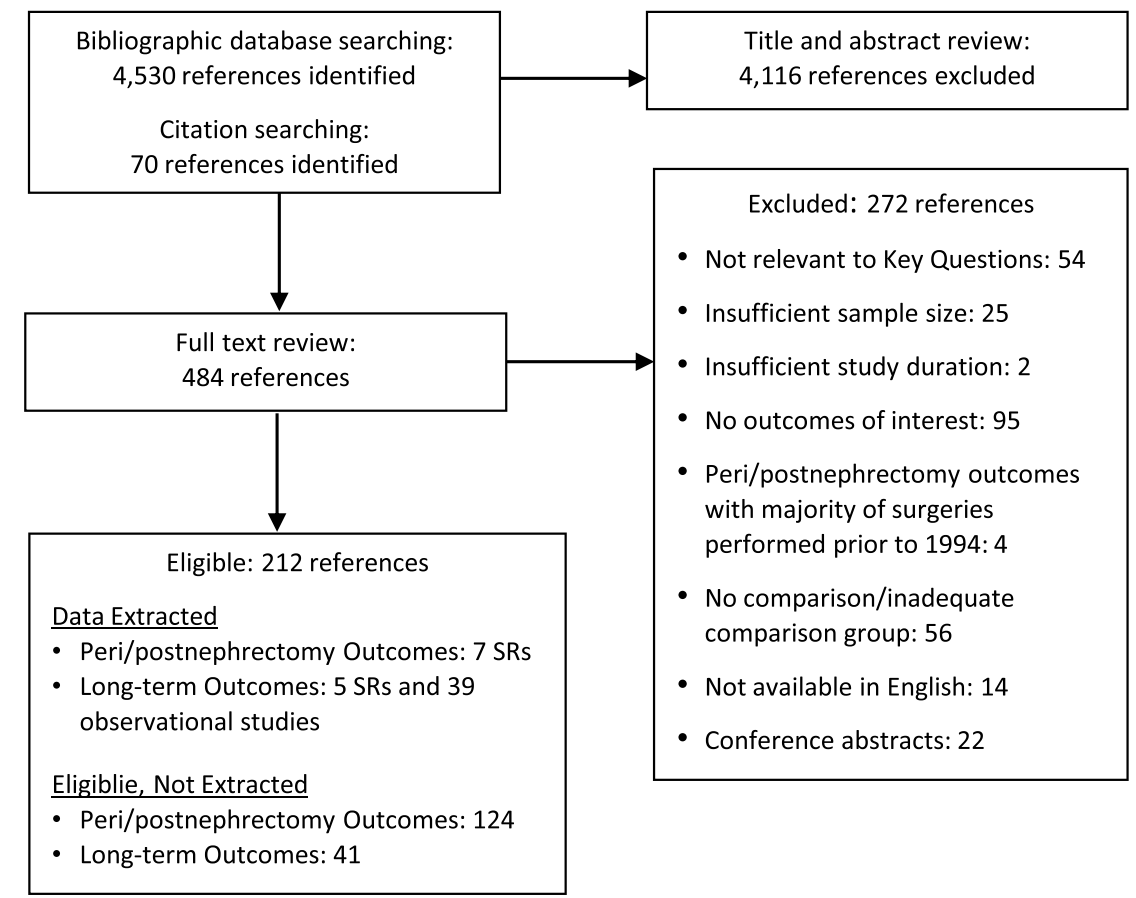

FIGURE 2. Literature flow diagram. SR, systematic review. 
http://links.lww.com/TP/B432 for perioperative/postoperative studies; Supplemental Appendix D, SDC, http://links.lww. com/TP/B432 for long-term outcomes studies of the evidence review publication; Evidence Report, SDC, http:// links.lww.com/TP/B434). Results were grouped by Key Question.

\section{De Novo Evidence Generation}

In developing the current guideline, the WG concluded that the framework for assessing the acceptability of candidates for living kidney donation needed to be restructured to include a comprehensive determination of risk to the donor, based on simultaneous consideration of a composite profile of risk factors. No previous guidelines have advocated for this approach to the evaluation of living donor candidates, and there is a paucity of data to demonstrate its feasibility and applicability. The WG therefore collaborated with the Chronic Kidney Disease-Prognosis Consortium (CKD-PC) to conduct a meta-analysis to produce a comprehensive riskprediction model for end-stage kidney disease (ESKD) applicable to donor candidates. The development and application of this clinical prediction tool are described in chapter 1 (Framework) of this guideline and in a separate publication. ${ }^{7}$

To inform choice of methods for kidney function estimation in the donor candidate evaluation, a web-based calculator was developed to compute posttest probabilities for measured glomerular filtration rate (mGFR) above or below threshold probabilities for decision-making based on estimated glomerular filtration rate (eGFR). The development and application of this clinical tool are described in chapter 5 (Kidney Function) of this guideline and in a separate publication. ${ }^{8}$

\section{Grading the Quality of Evidence and the Strength of Guideline Recommendations}

A structured approach, based on GRADE ${ }^{1,9,10}$ and facilitated by the use of evidence profiles, was used to grade the quality of the overall evidence and the strength of recommendations. For each topic with recommendations informed by the systematic review, the discussion of grading evidence quality was led by the ERT, and the discussion regarding the strength of the recommendations was led by the WG cochairs. The "strength of a recommendation" indicates the extent to which one can be confident that adherence to the recommendation will do more good than harm. The "quality of a body of evidence" refers to the extent to which confidence in an estimate of effect is sufficient to support a recommendation.

\section{Grading the Strength of the Recommendations}

The strength of a recommendation is graded as level 1 or level 2. Table 4 shows the KDIGO nomenclature for grading the strength of a recommendation and the implications of each level for patients, clinicians, and policy makers. Recommendations can be for or against doing something. Each recommendation includes an explicit link between the quality of the available evidence and the strength of that recommendation. However, the strength of a recommendation is determined not only by the quality of the evidence, but also by other, often complex judgments regarding the size of the net medical benefit (potential risks vs benefit), values, and preferences, and costs (Table 5). Formal decision analyses including cost analysis were not conducted.

\section{Grading the Overall Quality of Evidence}

The quality of the overall body of evidence was then determined on the basis of the quality grades for all outcomes of interest, taking into account explicit judgments about the relative importance of each outcome. The resulting 4 final categories for the quality of overall evidence were: “A," "B," "C," or "D" (Table 6).

\section{Ungraded Statements}

This category was designed to allow the WG to issue advice on topics not explicitly addressed in the systematic review. The current guideline is notable in that many clinically important topics in living donation are not ethically or practically amenable to randomized controlled study designs and have not been not addressed in controlled observational studies. Thus, many recommendations were generated on topics deemed important for the care of living donors that were not addressed by eligible studies in the systematic evidence review. These recommendations were developed using other literature and WG consensus, and are therefore 'ungraded.'

As a result, very few of the guideline recommendations were rated for strength of the recommendation and quality of the evidence. This is not to say that there was no evidence for such "ungraded" guideline recommendations, but the WG and ERT only graded recommendations that were included as part of the ERT's systematic review and fulfilled the a priori search inclusion criteria. The WG felt that such "good practice statements" were necessary to address important aspects of donor care, and their preponderance may

\section{TABLE 4.}

\section{KDIGO nomenclature and description for grading recommendations}

\begin{tabular}{|c|c|c|c|}
\hline \multirow[b]{2}{*}{ Grade $^{a}$} & \multicolumn{3}{|c|}{ Implications } \\
\hline & Patients & Clinicians & Policy \\
\hline Level 1 'We recommend' & $\begin{array}{l}\text { Most people in your situation would want } \\
\text { the recommended course of action and } \\
\text { only a small proportion would not }\end{array}$ & $\begin{array}{l}\text { Most patients should receive the } \\
\text { recommended course of action }\end{array}$ & $\begin{array}{l}\text { The recommendation can be evaluated as } \\
\text { a candidate for developing a policy or a } \\
\text { performance measure }\end{array}$ \\
\hline Level 2 'We suggest' & $\begin{array}{l}\text { The majority of people in your situation } \\
\text { would want the recommended course } \\
\text { of action, but many would not }\end{array}$ & $\begin{array}{l}\text { Different choices will be appropriate for } \\
\text { different patients. Each patient needs help } \\
\text { to arrive at a management decision consistent } \\
\text { with her or his values and preferences }\end{array}$ & $\begin{array}{l}\text { The recommendation is likely to require } \\
\text { substantial debate and involvement of } \\
\text { stakeholders before policy can } \\
\text { be determined }\end{array}$ \\
\hline
\end{tabular}

a The additional category 'Not Graded' is used, typically, to provide guidance based on common sense or where the topic does not allow adequate application of evidence. The most common examples include recommendations regarding monitoring intervals, counseling, and referral to other clinical specialists. Ungraded recommendations are generally written as simple declarative statements, but are not meant to be interpreted as being stronger recommendations than Level 1 or 2 recommendations. 
TABLE 5.

Determinants of strength of a recommendation

\begin{tabular}{|c|c|}
\hline Factors & Comments \\
\hline Balance between desirable a & $\begin{array}{l}\text { The larger the difference between the desirable and undesirable effects, the more likely a strong recommendation } \\
\text { is warranted. The narrower the gradient, the more likely a weak recommendation is warranted }\end{array}$ \\
\hline Quality of the evidence & The higher the quality of evidence, the more likely a strong recommendation is warranted \\
\hline Values and preferences & $\begin{array}{l}\text { The more variability in values and preferences, or the more uncertainty in values and preferences, the more likely a } \\
\text { weak recommendation is warranted. Values and preferences were obtained from the literature where possible. } \\
\text { or were assessed in the judgment of the work group where robust evidence was not identified }\end{array}$ \\
\hline Costs (resource allocation) & $\begin{array}{l}\text { The higher the costs of an intervention—-that is, the more resources consumed—the less likely a strong } \\
\text { recommendation is warranted }\end{array}$ \\
\hline
\end{tabular}

be attributed to numerous reasons as stated in Table $7 .{ }^{11}$ When the WG determined that there was evidence for a recommendation that was outside the scope of the ERT review, this was indicated in the rationale for that recommendation. It is also important to note that when recommendations from other KDIGO WGs were modified for the purpose of this guideline, the prior grading was provided in the rationale but the adapted recommendations were not graded so as to limit grading only to statements derived from the de novo systematic review performed for this guideline.

\section{Developing the Recommendations}

Draft recommendation statements were developed by the WG cochairs and WG members with input from all WG members. The health risks and benefits associated with each recommendation were considered when formulating the guideline, as well as information on patient preferences when available. Recommendation statements were revised in a multi-step process during teleconferences and 2 face-tofacemeetings, as well as in subsequent emails. All WG members provided feedback on initial and final drafts of the recommendations.

\section{Format for Recommendations}

Each chapter contains one or more specific recommendations. When pertinent evidence was available in the systematic review, the strength of recommendation is indicated as level 1 or level 2 and the quality of the supporting evidence is shown as A, B, C, or D. When the ERT search parameters did not identify evidence from eligible studies pertinent to a recommendation, the statement is ungraded. In all cases, recommendation statements and grades (if applicable) are followed by rationale text summarizing the key points of the evidence base and the judgments supporting the recommendations. Research recommendations for future work to help resolve current uncertainties are also outlined at the conclusion of each chapter.

\section{Limitations of Systematic Review Approach}

Although the literature searches were intended to be comprehensive, they were not exhaustive. Hand searches of journals were not performed, and review articles and textbook chapters were not systematically searched. However, any important studies known to domain experts that were missed by the electronic literature searches were added to retrieved articles and reviewed by the WG.

\section{Review of Guideline Development Process}

Several tools and checklists have been developed to assess the quality of the methodological process for systematic review and guideline development. These include the Appraisal of Guidelines for Research and Evaluation (AGREE 2) criteria, ${ }^{12}$ the Conference on Guideline Standardization (COGS) checklist, ${ }^{13}$ and the Institute of Medicine's recent Standards for Systematic Reviews ${ }^{14}$ and Clinical Practice Guidelines We Can Trust. ${ }^{15}$ Table 8 displays the criteria which correspond to the COGS checklist and how each one is addressed in this guideline.

\section{Public Comment and Revision}

A draft of the guideline was distributed for open public review in November 2015. The guideline was revised into final form by WG cochairs and members. A point-by-point response to all public comments is available online (Supplemental Appendix E, SDC, http://links.lww.com/TP/B433). All WG members approved the final version of the guideline.

\section{CHAPTER 1: GOALS OF EVALUATION, FRAMEWORK FOR DECISION-MAKING, AND ROLES AND RESPONSIBILITIES}

The ERT search parameters did not identify evidence from eligible studies pertinent to the recommendations in chapter 1 and therefore the following recommendations are "Not Graded."

\section{TABLE 6.}

Final grade for overall quality of evidence

\begin{tabular}{lll}
\hline Grade & Quality of evidence & \\
\hline A & High & We are confident that the true effect lies close to that of the estimate of the effect \\
B & Moderate & The true effect is likely to be close to the estimate of the effect, but there is a possibility that it is substantially different \\
C & Low & The true effect may be substantially different from the estimate of the effect \\
D & Very low & The estimate of effect is very uncertain, and often will be far from the truth \\
\hline
\end{tabular}


TABLE 7.

Reasons why many ungraded recommendations are issued in this guideline

The ERT's inclusion criteria (Table 2) are necessarily stringent given the limited time and resources for collecting and summarizing available evidence. As such, ungraded recommendations are issued:

- For topics that are not part of the ERT's inclusion criteria or when evidence lies outsides ERT's search yield

- In areas where the work group does not believe evidence is available (eg, recommendations grounded in ethics) or for which no there is no reasonable alternative (ie, based on common sense). Such recommendations may be deemed necessary because the work group considers such "good practice statements" essential especially when net benefit is great and unequivocal.

- In areas for which systematic review is not applicable (eg, monitoring intervals, counseling)

- Based on indirect evidence (eg, extrapolation of evidence from studies in general population)

- When adaped from prior KDIGO guidelines and extrapolated from different populations of interest (eg, general population or CKD population from KDIGO CKD Guideline)

CKD, chronic kidney disease; ERT, evidence review team; KDIGO, Kidney Disease: Improving Global Outcomes.

\section{Goals and Principles of Evaluation}

1.1: The donor candidate's willingness to donate a kidney voluntarily without undue pressure should be verified.

1.2: The benefits and risks of kidney donation should be assessed for each donor candidate.

1.3: The decision to accept or exclude a donor candidate should follow transplant program policies.

1.4: Donor candidate decision-making should be facilitated through education and counseling on individualized risks and benefits, methods to minimize risks, and the need for postdonation follow-up.

1.5: For an accepted donor candidate, a plan for donation care and follow-up should be formulated to minimize risks of donation.

1.6: For an excluded donor candidate, a plan for any needed care and support should be formulated.

\section{Framework for Decision-Making}

1.7: The donor candidate, the intended recipient, and the transplant program must all agree with the decision to proceed with donation in concordance with transplant program policies and informed consent.

1.8: Transplant program policies must be defensible based on current understanding of the risks and benefits of kidney donation, and should apply to all donor candidates evaluated at the center.

1.9: Each transplant program should establish policies describing psychosocial criteria that are acceptable for donation, including any program constraints on acceptable relationships between the donor candidate and the intended recipient.

1.10: All donor candidates should be evaluated using the same criteria, regardless of whether donation is directed towards a designated recipient.

1.11: Each transplant program should establish policies describing medical criteria that are acceptable for donation, addressing when possible, numeric thresholds for shortterm and long-term postdonation risks above which the transplant program will not proceed with donation. Risks should be expressed as absolute rather than relative risks.

1.12: When possible, transplant programs should provide each donor candidate with individualized quantitative estimates of short-term and long-term risks from donation, including recognition of associated uncertainty, in a manner that is easily understood by donor candidates.

1.13: Transplant programs should evaluate donor candidate risks in comparison to predetermined thresholds for acceptance. If a donor candidate's postdonation risk is above the transplant program's acceptable risk threshold, the risk is not acceptable for donation. If a donor candidate's postdonation risk is below the transplant program's acceptable risk threshold, the candidate makes the decision whether or not to proceed with donation.

1.14: If a donor candidate is not acceptable, the transplant program should explain the reason for nonacceptance to the donor candidate.

1.15: Transplant programs should protect donor candidate's privacy regarding the evaluation, including all considerations in the decision to donate or not.

\section{Roles and Responsibilities}

1.16: A multidisciplinary transplant program team knowledgeable in kidney donation and transplantation should evaluate, care for, and formulate a plan for donor care including long-term follow-up.

1.17: Transplant programs should minimize conflict of interest by providing at least one key team member not involved in the care or evaluation of the intended recipient who evaluates the donor candidate and participates in the determination of donor acceptance.

1.18: Transplant programs should conduct as efficient a donor evaluation as possible, meeting the needs of donor candidates, intended recipients and transplant programs.

\section{RATIONALE}

\section{Goals and Principles of Donor Evaluation}

Evaluation of candidates for living kidney donation requires balancing ethical principles of autonomy, beneficence, nonmaleficence, voluntarism and justice. ${ }^{16}$ Determining acceptability or nonacceptability of donor candidates requires an assessment of their potential risks and anticipated benefits of donation, independent of intended recipient issues. Donation must be voluntary (autonomous), and the motivation for donation must be altruistic - to satisfy a well-considered desire to help another person. There must be protection from undue pressure or coercion at every step in the evaluation and donation process, including the option to confidentially withdraw from the evaluation or to decline to donate at any time with the full support of the transplant program. ${ }^{17}$ In addition to these ethical principles, protection of patient privacy must be ensured. However, information regarding donor lifestyle, exposures or medical history that increase the risk for transmission of disease may need to be disclosed to the intended recipient for donation and transplantation to proceed; donor candidates should be given the opportunity to withdraw if they do not consent to sharing relevant personal health information in such circumstances. 
TABLE 8.

The Conference on Guideline Standardization (COGS) ${ }^{13}$ checklist for reporting clinical practice guidelines

Topic

(1) Overview material

(2) Focus

(3) Goal

(4) User/setting

(5) Target population

(6) Developer

(7) Funding source/sponsor Identify the funding source/sponsor and describe its role in developing and/or reporting the guideline

Disclose potential conflict of interest

(8) Evidence collection

Describe the methods used to search the scientific iterature, including the range of dates and databases searched, and criteria applied to filter the retrieved evidence

(9) Recommendation grading criteria

(10) Method for synthesizing evidence

(11) Prerelease review

(12) Update plan

(13) Definitions

(14) Recommendations and rationale

Provide a structured abstract that includes the guideline's release date, status (original, revised, updated), and print and electronic sources

Describe the primary disease/condition and intervention/ service/technology that the guideline addresses

Indicate any alternative preventative, diagnostic or therapeutic interventions that were considered during development

Describe the goal that following the guideline is expected to achieve, including the rationale for development of a guideline on this topic

Describe the intended users of the guideline (eg, provider types, patients) and the settings in which the guideline is intended to be used

Describe the patient population eligible for guideline recommendations and list any exclusion criteria

Identify the organization(s) responsible for guideline development and the names/credentials/potential conflicts of interest of individuals involved in the guideline's development

Describe the criteria used to rate the quality of evidence that supports the recommendations and the system for describing the strength of the recommendations.

Recommendation strength communicates the importance of adherence to a recommendation and is based on both the quality of the evidence and the magnitude of anticipated benefits and harms eg, evidence tables, meta-analysis, decision analysis

Describe how the guideline developer reviewed and/or tested the guidelines before release

State whether or not there is a plan to update the guideline and, if applicable, an expiration date for this version of the guideline of the guideline that might be subject to misinterpretation

State the recommended action precisely and the specific circumstances under which to perform it
Describe how evidence was used to create recommendations, For systematic review topics, summary tables and evidence

Define unfamiliar terms and those critical to correct application Abbreviations and Acronyms

Justify each recommendation by describing the linkage between the recommendation and its supporting evidence

Indicate the quality of evidence and the recommendation strength, based on the criteria described in Topic 9
Discussed in KDIGO Clinical Practice Guideline on the Evaluation and Care of Living Kidney Donors

Abstract and Methods for Guideline Development

This guideline seeks to formalize the framework for decision making and evaluation process for living kidney donor candidates and to define the optimal postdonation follow-up care

This Clinical Practice Guideline is intended to assist the practitioner in evaluating living kidney donor candidates and optimizing the care for donors and their quality of life

Target audience is practicing nephrologists, transplant physicians and other allied health professionals who work in the setting of living kidney transplantation

Living kidney donors

\section{Organization: KDIGO}

Names/credentials/potential conflicts of interest of individuals involved in the guideline's development are disclosed in the Biographic and Disclosure Information

This guideline is supported by KDIGO with contributions from Canadian Blood Services, Canadian Society of Nephrology, Minneapolis Medical Research Foundation, and The Transplantation Society

Topics were triaged either to a) systematic review, b) systematic search followed by narrative summary (eg, prior living kidney donor guidelines), or c) narrative summary. The search was updated through September 2014 and supplemented by articles identified by work group members through January 2017. We also searched for pertinent existing guidelines and systematic reviews

Quality of evidence and strength of recommendations were graded following the GRADE approach (Tables 4 and 6). The work group could provide general guidance in ungraded statements profiles were generated

The guideline had undergone external public review in November to December 2015. Public review comments were compiled and fed back to the work group, which considered the comments in its revision of the guideline The requirement for an update will be assessed on an ongoing basis from the publication date for potential important new evidence that may lead to changes to the recommendations

This guideline contains recommendations for evaluation of kidney donor candidates and postdonation follow-up care. Each recommendation builds on a supporting rationale with evidence tables if available. The strength of the recommendation and the quality of evidence are provided in parenthesis within each recommendation, where applicable 


\section{Topic}

(15) Potential benefits and harms

(16) Patient preferences

(17) Algorithm

(18) Implementation considerations
Description

Describe anticipated benefits and potential risks associated with implementation of guideline recommendations

Describe the role of patient preferences when a recommendation involves a substantial element of personal choice or values

Provide (when appropriate) a graphical description of the stages and decisions in clinical care described by the guideline

Describe anticipated barriers to application of the recommendations

Provide reference to any auxiliary documents for providers or patients that are intended to facilitate implementation

Suggest review criteria for measuring changes in care when the guideline is implemented
Discussed in KDIGO Clinical Practice Guideline on the Evaluation and Care of Living Kidney Donors

The benefits and harm for each recommendation are provided in summary tables and summarized in evidence profiles where applicable. The estimated balance between potential benefits and harm was considered when formulating the recommendations

The inclusion of patient values and preferences is clearly articulated where appropriate

No overall algorithm

Review criteria were not suggested because implementation with prioritization and development of review criteria must proceed locally. Furthermore, most recommendations are discretionary, requiring substantial discussion among stakeholders before they can be adopted as review criteria

Research recommendations were also outlined to address current gaps in the evidence base

GRADE, Grading of Recommendations Assessment, Development, and Evaluation; KDIGO, Kidney Disease: Improving Global Outcomes.

Preservation of donor candidate autonomy and minimization of short-term and long-term risks are high priorities in the practice of living donation. The transplant program has the responsibility to disclose anticipated risks and benefits to the donor candidate and intended recipient, tailored when possible for the characteristics of each donor candidate. ${ }^{18}$ The donor candidate must have adequate time to make an informed decision and must accept the need for long-term follow-up. The transplant program must offer support for decision-making through education and the informed consent process, and has a responsibility to confirm that the donor candidate understands the likely risks and benefits of donation. The transplant program makes the final determination of acceptance of the donor candidate, based on the program's policies. The transplant program must have a mechanism for resolving disagreement among team members regarding acceptability of donor candidates that avoids conflicts of interest.

\section{A Quantitative Framework for Equitable \\ Decision Making}

There will always be risks to living kidney donation. A central objective of donor candidate evaluation and selection is to minimize risks of short-term and long-term adverse outcomes after donation, and to ensure the risks are acceptable. Consistent, transparent and defensible decisionmaking to accept or decline a living kidney donor candidate has been limited by the lack of an evidence-based means to provide individualized, quantitative estimates of postdonation risk. Prior living kidney donor guidelines describe postdonation risk in relation to single predonation characteristics assessed in isolation, and generally agree on the single predonation characteristics that are associated with higher risks of poor postdonation outcomes. However, prior guidelines often differ on the recommended specific threshold for a characteristic that should be used to accept or decline living donor candidates, and are unclear about how values above or below the threshold alter the risk of postdonation outcomes. There have been several calls to improve the current status quo, and to support better shared decision making between donor candidates and their transplant professionals. ${ }^{16,19-21}$

An important advance is quantification of the combined impact of all of a donor candidate's predonation demographic (eg, age, sex, and race) and health characteristics at the time of evaluation (eg, kidney function, blood pressure [BP], body mass index [BMI], and so on) on the risk of serious adverse outcomes after donation. Serious postdonation adverse outcomes can be surgical, medical or psychosocial, and may occur during the perinephrectomy period, in a fixed period of long-term follow-up (eg, 15 years after donation), or for the remaining lifespan of the donor.

As described within this overall framework, a transplant program can use various methods to establish its threshold for acceptable outcomes after kidney donation. For example, if a transplant program decides a lifetime postdonation risk of kidney failure of up to $5 \%$ is acceptable, and if a candidate's projected risk is estimated to be above this threshold, the program should decline this candidate as a donor. Donor candidate autonomy does not overrule medical judgment and transplant professionals are ethically justified to decline a donor candidate when they believe the risk of poor postdonation outcomes is too high. ${ }^{22}$ A poor outcome can have a very negative impact on the donor, on their recipient, and on public opinions about living donation.

Each transplant program should strive to develop and communicate a quantitative threshold of "acceptable risk" for each serious postdonation adverse outcome it wishes to avoid. Thresholds should be both evidence-based and consensus-based, and there are various sources of evidence and processes by which consensus can be achieved. Once established, a threshold should be applied consistently and transparently for all donor candidates evaluated by a program (unless subsequently revised). When a donor candidate's estimated risk is below the acceptable risk threshold, the transplant program should accept a donor candidate, and it should be the candidate's decision whether or not to proceed with living kidney donation after being informed of the risks. When a candidate's estimated risk is above the 
acceptable threshold, the transplant program is justified in declining the candidate and can ground its decision in a quantitative framework (Figure 3).

During the development of this guideline we have advanced concepts and analyses to support this framework and approach. Here we discuss certain serious adverse outcomes and their amenability to quantitative risk estimation. We focus particularly on the postdonation development of kidney failure requiring dialysis or transplantation because it is a central outcome of a donor candidate's long-term risk. Finally, we describe the path for future work necessary to strengthen this framework, which includes the need for additional data.

\section{Perinephrectomy Outcomes}

The incidence of perioperative death after living kidney donation is low. The 90-day all-cause mortality in a recent United States (US) study of 80347 donors was reported to be approximately 1 in $3000(0.03 \%)$ based on 25 deaths. ${ }^{23}$ Similar rates have also been reported in other studies. ${ }^{24,25}$ Given the low incidence of perioperative mortality, estimates for predonation characteristics that alter the risk of perioperative death are imprecise. For example, in this same study, ${ }^{23}$ a predonation history of hypertension was associated with a 1 in 270 risk of 90 -day mortality. However, this estimate was based only on 2 observed deaths, and the estimate would have substantially changed if 1 more or less death was observed; the $95 \% \mathrm{CI}$ for the estimate was also wide, ranging from 1 in 75 to 1 in 2220 . Thus, even if a transplant program defines an acceptable risk threshold for perinephrectomy mortality (for example, an incidence less than 1 in 1000), it will be difficult at this time to reliably determine a given donor candidate's estimated risk of this outcome according to their profile of predonation characteristics.

With respect to perioperative complications, the ERT identified 2 systematic reviews that examined perinephrectomy outcomes in relation to demographic and health characteristics of accepted donors. The ERT rated the quality of this evidence as very low (Evidence Report Tables 6 and 7, SDC, http://links.lww.com/TP/B434). In one review, a group of selected older donors (mean age, 66 years; range,
60 to 85 years at donation) did not differ statistically from a group of younger donors in their operative time, intraoperative blood loss, and length of hospital stay. ${ }^{26}$ In both reviews, groups of selected obese donors (mean BMI of $34.5 \mathrm{~kg} / \mathrm{m}^{2}$; range $32-39 \mathrm{~kg} / \mathrm{m}^{2}$ ) did not differ statistically from groups of nonobese donors in their rates of perioperative complications, operative time, blood loss and length of hospital stay. ${ }^{26,27}$

Since then a large US study examined predonation characteristics associated with a higher risk of donor nephrectomyrelated complications (as assessed through administrative data rather than adjudication, using a composite outcome of digestive, respiratory, procedural, urinary, hemorrhage, infectious or cardiac complications). ${ }^{28}$ In this study, where each donor candidate characteristic was considered by itself (rather than as a combination of characteristics), complication rates were higher in men versus women $(9.6 \%$ vs $7.2 \%)$; among African Americans (10.4\%) and whites $(8.7 \%)$ compared with other racial groups $(6.3 \%)$; among donors without private insurance $(8.5 \%)$ compared with those who had private insurance $(7.3 \%)$; and among donors with hypertension $(17.7 \%)$ compared with those without hypertension $(7.9 \%)$.

A subsequent study integrated national US donor registry data from 2008 to 2012 with administrative records from a consortium of 98 academic hospitals and found that $16.8 \%$ of donors experienced a diagnosis or procedure for a perinephrectomy complication, most commonly gastrointestinal $(4.4 \%)$, bleeding $(3.0 \%)$, respiratory $(2.5 \%)$, and surgical/anesthesia-related injuries $(2.4 \%) .{ }^{29}$ Major complications, defined as Clavien severity level 4 or 5, were identified in $2.5 \%$ of donors. After adjustment for demographic, clinical (including comorbidities), procedure, and center factors, compared with white donors, African Americans had significantly higher risks $(P<0.05)$ of experiencing any complication $(18.2 \%$ vs $15.5 \%)$ and of experiencing major complications $(3.7 \%$ vs $2.2 \%)$. Other significant correlates of major complications included obesity, predonation blood disorders, psychiatric conditions, and robotic nephrectomy, while greater annual hospital volume predicted lower risk.

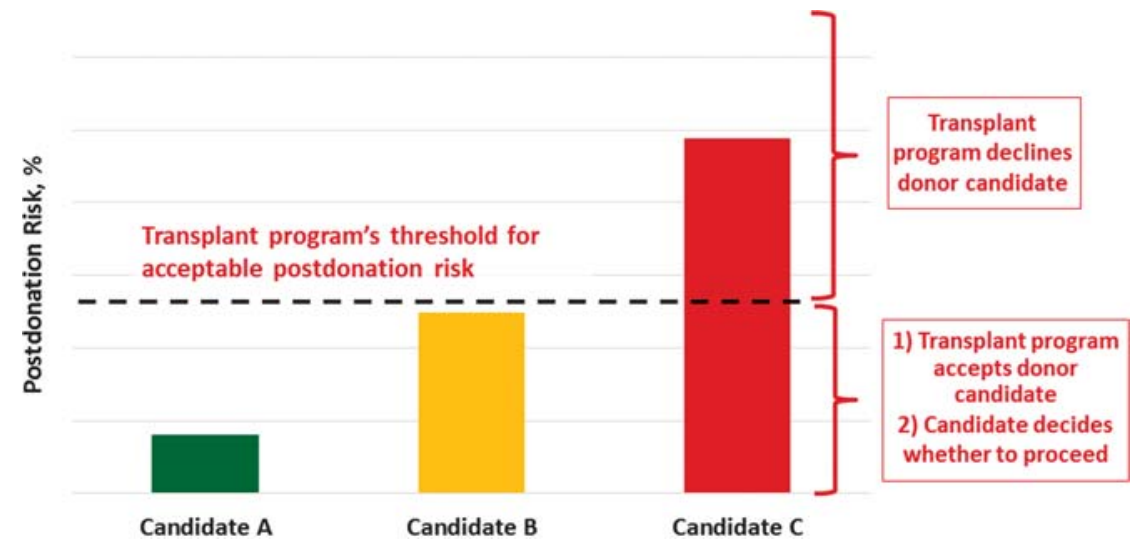

FIGURE 3. Framework to accept or decline donor candidates based on a transplant program's threshold of acceptable postdonation risk. The decision by the transplant program to accept or decline a donor candidate is grounded on whether an individual's estimated postdonation risk is above or below the threshold set (dotted line) by the transplant program. The threshold may vary across transplant programs, but the same threshold should apply to all donor candidates at each program. For example, candidate A (green) would be acceptable because the estimated projected postdonation risk is far below the threshold. Candidate B (yellow) could be accepted with caution because the estimated projected postdonation risk is close but below the threshold, and candidate $\mathrm{C}$ (red) would be unacceptable because the estimated postdonation projected risk is far above the threshold. 
As future data become available, it may become possible for transplant programs to estimate the risk of well-defined, serious perioperative complications according to a donor candidate's individual profile of baseline characteristics, and to compare these estimates to a threshold of acceptable risk to inform donor acceptance decisions.

\section{Long-term Outcomes}

Donating a kidney is a decision with lifetime implications for the donor. While there are many outcomes to consider after kidney donation, a central outcome directly related to having one kidney removed is the long-term risk of developing kidney failure requiring dialysis or transplantation, commonly referred to as ESKD. Donor candidates often have a good understanding of the health effects of kidney failure, as their reason to donate is to treat the kidney failure of their intended recipient. For these reasons, we have grounded a quantitative framework for medical evaluation and acceptance of donor candidates on the long-term risk of postdonation kidney failure.

Each donor candidate has a long-term risk (cumulative incidence) of developing kidney failure that is influenced by the combination of risks conferred by their demographic and health characteristics at the time of evaluation plus risk attributable to donation (Figure 4). Demographic characteristics include age, sex, and race. Health characteristics include glomerular filtration rate (GFR), albuminuria, BMI, $\mathrm{BP}$, diabetes status, smoking history, family history of kidney disease, and other factors. The risk attributable to donation may also vary according to demographic and health characteristics. Minimizing the lifetime risk of kidney failure in accepted donors is important to safeguard the practice, regardless of the degree to which it can be established that donation contributed to the risk of kidney failure.

Challenges to determining the postdonation lifetime risk of kidney failure based on current studies include limitations of study follow-up (the largest studies followed most donors for less than 2 decades rather than for their lifetime). ${ }^{30}$ The risk of kidney failure after donation is nonlinear, and is expected to be higher later ( $\geq 10$ years) than earlier ( $<10$ years) after donation. ${ }^{31}$ When the WG was convened, 2 recent studies reported that the risk of kidney failure is higher in donors compared with risk among nondonors with similar baseline demographics. The ERT assessed the quality of evidence from these 2 studies as moderate (Table 2 of Slinin et $\left.\mathrm{al}^{3}\right) .{ }^{30,32}$ Available data suggest that the average donationattributable risk of kidney failure is approximately 27 per $10000(0.3 \%)$ at 15 years, ${ }^{30}$ but there is substantial uncertainty in the estimate, and there are not sufficient data to project lifetime donation-attributable risk. Furthermore, the extent to which donation-attributable risk varies according to individual health characteristics is not known, ${ }^{33,34} \mathrm{al}$ though available evidence suggests there is higher donationattributable risks in some subgroups, such African Americans compared with white donors. ${ }^{30}$

Existing large population-based studies can help estimate the long-term risk of treated kidney failure in the absence of donation, based on a candidate's predonation health characteristics. Furthermore, if the risk of kidney failure attributable to donation becomes more precisely understood in relation to an individual's profile of baseline characteristics, then demographic-related, health status related, and donation-attributable risks can be aggregated to project individualized estimates of long-terms risks of postdonation kidney failure.

To help advance this paradigm, we enlisted the help of the CKD-Prognosis Consortium (CKD-PC) to develop a tool to project the 15-year and lifetime incidence of kidney failure in the absence of donation based on demographic and health characteristics at the time of evaluation in low-risk persons from large population cohorts. CKD-PC is a research group composed of investigators who analyze large cohort data and perform collaborative meta-analyses. The methods and results of these analyses are reviewed briefly here and

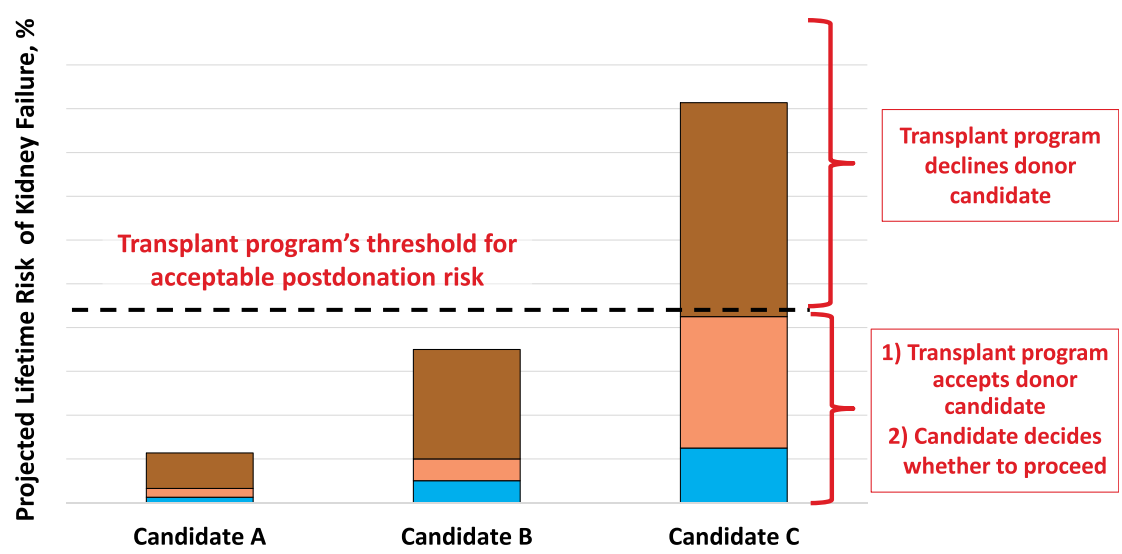

Donation-attributable risk (may vary by demographic and health characteristics)

Aggregate risk related to health characteristics in the absence of donation

(e.g. GFR, blood pressure, BMI, smoking)

Demographic-related (age, sex, race) risk in the absence of donation

FIGURE 4. Framework to accept or decline donor candidates based on a transplant program's threshold of acceptable projected lifetime risk of kidney failure, quantified as the aggregate of risk related to demographic and health profile and donation-attributable risks. The decision by the transplant program whether to accept or decline a donor candidate is grounded on the candidate's estimated postdonation lifetime risk, including estimated risk in the absence of donation (risk related to demographic and health characteristics as denoted in blue and beige, respectively) and estimated risk attributable to donation (brown). BMl, body mass index; GFR, glomerular filtration rate. 
presented in expanded form in a separate publication. ${ }^{7}$ To project the estimated long-term incidence of kidney failure among persons who do not donate a kidney according to 10 demographic and health characteristics, risk associations were derived from a meta-analysis of 7 general population cohorts. Relative risks were calibrated to the population-level annual incidence of ESKD in the United States, derived from actual ESKD incidence and mortality data collected by the US Renal Data System and overall mortality data from the US Census. ${ }^{35}$ Fifteen-year projections were compared with the observed risk among 52998 living kidney donors in the United States. For estimation of relative risks related to health characteristics, a total of 4933314 participants from 7 cohorts were followed for a median of 4 to 16 years. For a 40-year-old person with health characteristics similar to those of age-matched kidney donors, the 15 -year projections of ESKD risk in the absence of donation varied according to race and sex; the risk was $0.24 \%$ among black men, $0.15 \%$ among black women, $0.06 \%$ among white men, and $0.04 \%$ among white women. Risk projections were higher in the presence of lower eGFR, higher albuminuria, hypertension, current or former smoking, diabetes, and obesity. In the model-based lifetime projections, the risk of ESKD was highest among persons in the youngest age group, particularly among young black persons. The 15 -year observed postdonation risks among kidney donors in the United States were 3.5 to 5.3 times as high as the projected risks in healthy persons in the absence of donation, according to sex and race.

This study has important limitations. ${ }^{36}$ First, the projections were calibrated to historical incidence rates of ESKD from US population data. Annual incidence was derived with the use of life-table methods, which assume a constant age-, sex-, and race-specific incidence of ESKD over periods of decades and a static population substructure. Second, information on certain health characteristics of interest was not available, including heritable and environmental factors. The estimates reflect population averages for unmeasured characteristics. Donor candidates with a family history of kidney disease (especially younger candidates with such history) would be expected to have a higher risk of ESKD than projected. Third, the relative risk estimates were based on low-risk cohorts followed for a median of 4 to 16 years, based on an assumption of proportional hazards and after testing for nonproportionality. The analysis does not include untreated low GFR as an outcome, a condition that is more common among older persons, nor did it assess the risk of other outcomes, such as hypertension or preeclampsia, that have been linked to kidney donation. Finally, the analysis did not estimate the age at which ESKD would be expected develop in a donor candidate or the duration of ESKD before death.

The resulting risk models were incorporated into an online risk prediction tool for 15-year and lifetime ESKD risk (http://www.transplantmodels.com/esrdrisk/). Although the risk tool was developed specifically for the United States, the methods used may be adapted to other countries with the availability of local data sources. The WG endorses use of the online tool as the foundation for a new evaluation framework centered on simultaneous consideration of multiple demographic and health characteristics to predict long-term risk of an important outcome, while recognizing limitations in precision of risk estimates and uncertainty in donation-attributable risk. These models should be refined through further research to improve the precision and generalizability of predonation risk estimates and to incorporate estimates of indivdualized risk attributable to donation.

We endorse a quantitative framework for donor candidate evaluation and acceptance centered on lifetime risk of postdonation kidney failure (Table 9). For example, if a transplant program sets the acceptable lifetime postdonation ESKD risk threshold at 5\%, and assumes a donationattributable RR of 3.5 to 5.3 according to sex and race, then the acceptable predonation lifetime ESKD risk threshold would be approximately $1.0-1.5$. This strategy enables decision-making based on a more comprehensive and integrated assessment of risk factors than is currently practiced, but application of the currently available online tool at this time requires clinician insight and interpretation. ${ }^{37}$ Currently, there remains uncertainty in lifetime risk estimates, particularly for younger donor candidates, donors from developing countries, and ethnicities other than black or white race. At this time, transplant programs and donor candidates may consider other factors in their acceptance criteria for living kidney donation in addition to quantitative risk estimates. We see this work as starting point, and advocate strongly for continued efforts to improve the precision, tailoring and generalizability of predonation and postdonation risk estimates. Quantifying donation-attributable risk according to predonation demographic and health profile is a leading priority for future research, and we will update the online tool once estimates are available.

In summary, we advocate for a quantitative paradigm wherein transplant programs accept or decline donor candidates using the strongest evidence-based criteria currently available to simultaneously consider a profile of risk factors (demographic and health characteristics at evaluation and risk attributable to donation) and support consistent, transparent and defensible decisions. Ongoing efforts are needed to strengthen and advance this paradigm, including incorporation of data from cohorts observed for longer periods of time (ideally over the lifetime) and from diverse countries; estimation of risks related to genetic and familial factors; and quantification of donation-attributable risks

\section{TABLE 9.}

Approaches to implementation of a quantitative framework for donor candidate medical evaluation and acceptance centered on lifetime risk of kidney failure

(1) Use the online tool (http://www.transplantmodels.com/esrdrisk) to estimate the projected lifetime risk of kidney failure in the absence of donation according to baseline demographic and health characteristics included in the online tool

(2) Multiply the projected predonation risk by the best available estimate for donation-attributable risk to obtain the projected postdonation risk. For example, Grams et al ${ }^{7}$ report a relative risk of 3.5 to 5.3 for $15-y$ end-stage kidney disease risk, according to sex and race

(3) Compare the projected risk estimate to the program's postdonation threshold of acceptable risk

(4) Exercise caution when there is concern that the individual has risk factors not captured in the online tool (eg, familial or genetic risk) and for younger candidates 


\section{TABLE 10.}

Roles and responsibilities of participants in donor candidate identification, evaluation, care, and follow-up

Entity
General nephrologist/dialysis unit/advanced chronic
kidney disease clinic

Transplant program

\section{Recipient candidate health insurance carrier}

Donor/donor candidate health insurance carrier

Donor/donor candidate primary care physician

Donor/donor candidate physician/nephrologist

Donor surgeon

Nurse coordinator

Dietitian

Social worker/psychologist/psychiatrist

Independent living donor advocate ${ }^{a}$

Regulatory and oversight agencies

Donor/donor candidate

\section{Responsibilities}

- Educate recipient candidates regarding early referral, preemptive and living donor transplantation options and resources for identifying donor candidates

- Educate recipient candidates regarding early referral, preemptive and living donor transplantation options

- Educate donor candidates regarding all phases of the donation process including evaluation, surgery, postdonation follow-up, expected risks and outcomes, and existing uncertainties

- Provide coverage for costs related to recipient candidate evaluation and transplantation, including coverage of donor candidate evaluation and treatment costs

- Educate donor candidates regarding any anticipated out-of-pocket expenses related to evaluation and donation processes

- Educate donor candidates regarding coverage for postdonation complications, both short-term and long-term after donation

- Support donor candidates in their desire for information to make informed decisions

- Participate in predonation and early postdonation care as needed

- Participate in long-term care after donation

- Evaluate donor candidates without influence from recipient considerations

- Oversee evaluation testing

- Provide education regarding all phases of the donation process including postdonation follow-up

- Participate in donor candidate selection

- As needed, participate in care during the surgical hospitalization, in the early postdonation period, and long-term after donation, including serving as resource for primary providers

- Evaluate donor candidates for surgical risks and plan surgical approach

- Provide education on the surgical procedure, risks and expected recovery

- Participate in donor candidate selection

- Provide care during surgery and perioperative period, and as needed postdonation

- Educate donor candidates on recipient candidate treatment options and phases of the donation process

- Facilitate and oversee completion of the evaluation

- Assist with arranging surgery and inpatient care

- Arrange and oversee postoperative care, and coordinate a plan for postdonation follow-up

- Review dietary habits and metabolic status including measures of obesity when needed

- Provide guidance for nutritional treatment if indicated, including recommendations to address obesity

- Perform donor candidate psychosocial evaluation including assessment of motivation

- Educate donor candidates on recipient candidate treatment options

- Provide donor candidates with information and support services related to donation, including information on resources that may be available to assist with donation-related expenses

- Discuss potential adverse outcomes including loss of income because of donation, donation-related complications, or failure of the transplant. Assess the ability of donor candidates to cope with adverse outcomes

- Support informed donation decisions

- Assist donors with planning support around the time of donation and creation of a long-term follow-up plan

- Verify that donor candidates have information needed to make a voluntary and informed decision on whether or not to donate

- Verify consent for donation

- Function independently from the recipient candidate's team

- Advocate for the rights of donor candidates and donors

- Create policies for minimum standards of donor candidate informed consent, evaluation, care and donor follow-up

- Agree to required psychosocial and medical evaluation

- Agree to disclosure of required personal health information to intended recipient with regard to risk of disease transmission when necessary, or to withdraw from donation

- Agree to required lifestyle modifications to reduce risks of donation and promote long-term good health

- Agree to participate in postdonation follow-up

\footnotetext{
${ }^{a}$ The role of the independent donor advocate may be served by another team member as long as criteria for independence and advocacy functions are satisfied.
} 
according to multiple predonation health characteristics. The scope of the current guideline is focused on donor safety, and excludes consideration of recipient outcomes based on donor characteristics. However, we appreciate that a donor candidate's profile of predonation characteristics may also have important impacts on posttransplant recipient outcomes, and that topic also warrants future consideration.

\section{Roles and Responsibilities}

Transplant programs bear the primary responsibility for evaluation, care and follow up of living kidney donor candidates and donors. The main responsibilities of the transplant program are to establish and maintain policies and a team of professionals to provide care according to policies. However, many other entities share in these responsibilities (Table 10). The decision to donate should be regarded as a shared responsibility between the donor candidate, the donor candidate's primary physician, and the transplant program. Transplant programs and the organizations that regulate transplant practice should:

- Evaluate and disclose risks to the best of currently available knowledge

- Respect the donor candidate's autonomy, including autonomy to take risk, within a program's/regulators' upper bound of acceptable risk

- Embrace a long-term relationship with the donor, because some risks are uncertain or evolving

\section{RESEARCH RECOMMENDATIONS}

- Strengthen and refine estimates of long-term projected predonation and postdonation risks of kidney failure, including incorporation of data from cohorts observed for longer periods of time (ideally over the lifetime), from different countries and regions, and estimates of risk related to familial, genetic (eg, apolipoprotein L1 [APOL1]), and other factors (eg, birth weight).

- Engage in consensus-building activities among transplant professionals, donors and recipients to help establish uniform threshold of unacceptable risk. Strategies that may help inform consensus include:

- Estimate the long-term risk of kidney failure among previously accepted donors, such as those captured in large national databases.

- Estimate long-term risk of kidney failure based on donor acceptance criteria specified in prior guidelines.

Evaluate implications of racial variation in long-term risk of kidney failure exceeding possible thresholds for acceptable risk on opportunities for living donation. Develop strategies to promote equitable access to kidney transplantation without placing certain donors at unacceptable risk.

- Determine the best methods of communicating individualized risks to donor candidates and their intended recipients so that the information is understood and supports patient decision-making.

- Explore application of individualized risk estimates to guide predonation support and counseling (eg, target predonation BMI levels) to minimize the risk of adverse postdonation outcomes.

- Develop tools to predict risks of adverse short- and long-term psychosocial outcomes and a broader spectrum of medical outcomes according to predonation characteristics.

\section{CHAPTER 2: INFORMED CONSENT}

The ERT search parameters did not identify evidence from eligible studies pertinent to the recommendations in chapter 2 and therefore the following recommendations are "Not Graded."

\section{Process of Informed Consent}

2.1: Informed consent for donation should be obtained from the donor candidate in the absence of the intended recipient, family members and other persons who could influence the donation decision.

\section{Capacity for Decision Making}

2.2: The donor candidate's capacity to provide informed consent (ie, ability to understand the risks, benefits and consequences of donation) should be confirmed before proceeding with evaluation and donation.

2.3: Substitute decision makers should not be used on behalf of a donor candidate who lacks the capacity to provide informed consent (eg, children or those who are mentally challenged), except under extraordinary circumstances and only after ethical and legal review.

\section{Content of Disclosure}

2.4: Protocols should be followed to provide each donor candidate with information on:

- The processes of evaluation, donor acceptance, and follow-up

- The types of information that may be discovered during the evaluation, and what the transplant program will do with such information

- Individualized risks, benefits and expected outcomes of the donor evaluation, donation, and postdonation health, including a discussion of the uncertainty in some outcomes

- Treatment alternatives available to transplant candidates, and average expected outcomes

- How personal health information will be handled

- Availability of transplant program personnel for support

\section{Comprehension of Disclosed Information}

2.5: The donor candidate's understanding of the relevant information on the risks and benefits of donation should be confirmed before proceeding with donation.

\section{Voluntarism}

2.6: Donor candidates should have adequate time to consider information relevant to deciding whether they wish to donate or not.

2.7: A donor candidate's decision to withdraw at any stage of the evaluation process should be respected and supported in a manner that protects confidentiality.

2.8: A donor candidate who decides not to donate and has difficulty communicating that decision to the intended recipient should be assisted with this communication by the transplant program.

\section{RATIONALE}

Obtaining informed consent to be evaluated as a living kidney donor candidate, and informed consent to undergo living kidney donation, are processes rather than a discreet 
event. The transplant program has a responsibility to establish that the donor candidate is capable of understanding the relevant information (capacity), is adequately informed of the likely risks and benefits of the donation, and of the alternative treatment options available to the recipient (disclosure), understands this information (understanding), and is acting voluntarily (voluntarism). This chapter provides recommendations to ensure satisfaction of the informed consent requirements for the living kidney donor candidate. The reader should also refer to chapter 1 for related discussions on the framework for decision-making and chapter 18 on the ethical, legal, and policy framework of living donation. Details of specific donation-related surgical, medical, and psychosocial risks are provided in other chapters (3-17) of the guideline.

\section{Process of Informed Consent}

Transplant programs must establish a defensible process to ensure that the requirements of informed consent are met. ${ }^{18,38-41}$ To date, donor candidate informed consent processes have been shown to vary widely across transplant programs worldwide, with discrepancies noted in standards, consistency and implementation. ${ }^{39,40,42-45}$ It has been recommended that the informed consent structure and process be the same for donor candidates regardless of relationship (or lack thereof for nondirected donation) between the donor candidate and their intended recipient. ${ }^{39,46,47}$

Transplant programs must assure a donor candidate is acting voluntarily and not yielding to pressure or coercion. It is best if evaluations of the donor candidate and the intended recipient are performed by separate teams to mitigate potential conflict of interest. A recommendation that the donor candidate be evaluated by a team that is independent of the evaluation of the intended recipient is also recommended in several past guidelines. ${ }^{47-51}$ The process of informed consent with a living kidney donor candidate should include discussions with a healthcare professional skilled and knowledgeable in organ donation and in evaluating a person's comprehension of the information. In the United States, to minimize conflict of interest, living donor recovery hospitals must designate and provide each donor candidate with an Independent Living Donor Advocate who is independent of the intended recipient's evaluation and the decision to transplant the intended recipient. This person seeks to ensure that the rights of the donor candidate are protected, that all the requirements of informed consent are met, and that the donation decision is made voluntarily. ${ }^{51}$ Other countries may use other strategies such as an external review of planned donations to ensure that independence, advocacy for the donor's rights, and voluntarism are respected. ${ }^{52}$ While avoidance of conflict of interest is a central principle, it also remains important that healthcare professionals in the teams evaluating the donor candidate and intended recipient work together to ensure effective communication and coordination of the donation and transplant processes. For example, it would be inefficient to fully evaluate a donor candidate if their intended recipient does not meet eligibility criteria for transplantation.

\section{Capacity for Decision Making}

The transplant team has a duty to confirm that the donor candidate has the capacity to provide informed consent, and is able to communicate their decisions based on accurate comprehension of the information disclosed to them. ${ }^{2,18}$ Local laws and guidelines should be followed regarding minimum age criteria to become a living kidney donor. ${ }^{53}$ For example, prior guidelines indicate that persons who are younger than 18 years or who lack the mental capacity to provide informed consent should not become living kidney donors with the assistance of substitute decision-makers, and that donation in such a setting only be considered in highly exceptional circumstances (eg, young parent to child) after ethical and legal review. $18,38,47,48,51,54,55$

\section{Content of Disclosure}

Transplant programs must have a process to communicate relevant information to donor candidates that enables informed decision making. ${ }^{18}$ Some prior guidelines have suggested use of a standardized checklist to ensure that all items are disclosed. ${ }^{50}$ For US programs, the Organ Procurement and Transplantation Network (OPTN) has developed an informed consent checklist for programs to support compliance with policy requirements ${ }^{56}$ and a patient resource (English and Spanish translations) to explain the process in lay language for donor candidates. ${ }^{57}$

The donor candidate needs to be informed from the onset of what is involved in the donor evaluation, including the required assessments and anticipated timelines. In general, education about the process and potential outcomes of living donation should be introduced in a manner conducive to learning and understanding. Prior guidelines and policy statements have recommended that discussions be provided in a language that enables meaningful dialogue between the donor candidate and transplant program staff, using communication strategies and materials that are culturally sensitive. $^{47,49}$ The information should also be presented in a sympathetic environment, using simple language, allowing time for questions, with information that is appropriate to a candidate's understanding and experience, at a pace determined by their needs. ${ }^{49}$ Repetition of key information, and use of approaches that foster adult learning, are prudent. ${ }^{58}$ The information to be disclosed to the donor candidate is described in many prior guidelines and policy statements. ${ }^{47,48,51,54,59}$ Some regulations require minimum content elements that must be disclosed in the informed consent process, ${ }^{51}$ and these requirements should be respected when locally applicable. The donor candidate must also provide consent for some tests performed during the evaluation, such as consent to receive intravenous contrast for renal imaging. In this guideline, we present a list of the content of recommended disclosures during the donor candidate evaluation, considering these prior guidelines and policies (Table 11). Candidates should also be reminded that they can only donate a kidney as a living person once, even though they may know someone else who may need a kidney transplant in the future.

Treatment alternatives available to transplant candidates (in general terms, not with specific recipient medical information) should be disclosed to the donor candidate (Table 11). Donor candidates who are biologically incompatible with an intended recipient should be informed of the availability desensitization protocols and kidney paired donation (KPD), and the considerations related to pursuit of these treatment options. Logistics, outcomes and risks specific to KPD and planned incompatible transplantation are discussed in chapter 3 of this guideline. Participation in KPD and incompatible 
TABLE 11.

\section{Recommended content of disclosure during the evaluation of living donor candidates}

Type of disclosure

Handling of donor candidate's personal

health information

Risks of discovery of donor health information

Risk and expected outcomes of donation

Treatment alternatives available to transplant candidates

Process of transplant candidate selection and when the intended recipient's personal health information is shared with the donor candidate

Processes of donor candidate evaluation, candidacy determination, and follow-up

\section{Information disclosed to the donor candidate}

- Personal health information collected during the donor candidate evaluation is confidential and protected under privacy law, similar to other personal health information

- The transplant program will only disclose a donor candidate's personal health information to the intended recipient or other parties with the donor candidate's permission

- The donor candidate may be asked for permission to disclose certain personal health information to their intended recipient. This information may include the donor candidate's identity, immunonlogical compatibility, and medical history affecting the risk of disease transmission

- The program's policy for disclosing information and arranging follow-up care for each of the following: - A health condition that may require further medical intervention

- A health condition that could affect the donor candidate's ability to obtain insurance (eg, life, medical, disability), or the cost of insurance

- An infectious disease that must be reported to public health authorities

- A misattributed biological relationship between the donor candidate and intended recipient (such as misattributed paternity in a father-child relationship) discovered through blood group and immune compatibility testing

- The anticipated medical, surgical, psychosocial, and economic risks and outcomes of donation (for specific details see other chapters of this guideline), and the uncertainty in estimating risk and outcomes

- Treatment options for kidney failure, including dialysis and deceased donor kidney transplantation, and their average expected outcomes compared with living kidney donor transplantation

- Transplant candidate evaluation teams determine eligibility to receive a kidney transplant based on program criteria and clinical judgment

- Personal health information collected during the transplant candidate's evaluation is confidential, protected under privacy law, and is not generally shared with the donor candidate unless: 1) the transplant program determines the donor candidate requires such information to make an informed decision about proceeding with donation, and 2) the intended recipient gives permission for this information to be shared with the donor candidate

- Separate consents may be needed for some tests

- Programs and personnel available to help donors with the financial burden of donation

- It may be a crime to receive any valuable consideration (money, property) for donation

- A description of what will happen if the candidate decides not to donate, emphasizing the right of the candidate to decline to donate at any time with the full support of the transplant program

- The transplant program decides if the donor candidate is eligible for donation based on the results of their evaluation

- If excluded from donation, information on why the donor candidate does not meet the program's criteria for donation and how the transplant program will support the candidate

- The program's recommendations for follow-up care, including the timing and financial impacts of care and the need for regular, ongoing healthcare maintenance and healthy lifestyle choices

- The program's need to collect ongoing personal health information after donation to inform the care of the recipient, and to guide the care of the donor

- The program's policy about providing care to the donor after evaluation and donation

- The availability of national and regional policies to assure prompt access to dialysis and transplantation for living donors who develop kidney failure transplantation requires specific informed consent, and minimum content elements may be specified by paired donation programs. ${ }^{60,61}$ The transplant program should inform the donor candidate of policies regarding confidentiality and anonymity in KPD and nondirected donation, and should ensure donor acceptance of these policies before donation. ${ }^{62}$ Some programs now permit biologically compatible pairs to participate in KPD; when this option is discussed, donor candidates who are biologically compatible with their intended recipient should voluntarily decide whether or not to pursue this option. ${ }^{63-65}$

Participating in donor evaluation includes risks of discovery. These risks include possible discovery of a health condition that requires referral for further care, discovery of a health condition that could affect the donor candidate's insurability or cost of insurance, or discovery of an infectious disease for which there is a reporting requirement to public health authorities. Transplant programs should establish policies for managing such discoveries, and share these policies with the donor candidate as part of the informed consent process for evaluation. Testing a donor candidate and intended recipient in a family for the purpose of assessing immune compatibility may identify misattributed biological relationships as an incidental finding. For example, misattributed paternity is estimated to occur in approximately 1 to $3 \%$ of fatherchild living kidney donor-recipient pairs, or approximately 
$0.25 \%$ to $0.5 \%$ of all living kidney donation evaluations. ${ }^{66}$ Transplant programs should establish a policy on how or whether this information is disclosed. ${ }^{67,68}$

The donor candidate should be informed of donationrelated surgical, medical, and psychosocial outcomes and risks as provided in other chapters of this guideline, individualized whenever possible for donor characteristics, and including the uncertainty of estimates. As described in chapter 1, 90-day all-cause mortality after donation in a US study of 80347 donors (1994-2009) based on registry data and national death records was approximately 1 in $3000(0.03 \%) .{ }^{23} \mathrm{Sim}$ ilar rates have also been reported in other studies. ${ }^{24,25}$ Given the low incidence of perinephrectomy mortality, estimates for predonation characteristics that alter the risk of perioperative death are imprecise.

The donor candidate should be informed of anticipated recipient outcomes associated with living donor transplantation (such as 1-year, 5-year and median recipient graft and patient survival), and with available treatment alternatives including deceased kidney donor transplantation and different types of dialysis. US policy requires that donor candidates are provided with current national and program-specific 1-year transplant recipient patient and allograft survival statistics, and that donor candidates are also informed that any transplant candidate may have risk factors for increased likelihood of adverse outcomes (including graft failure, complications, mortality) that exceed local or national averages. ${ }^{51}$

Many regions have legislation that protects the confidentiality of personal health information, and the same protections apply to information collected during the donor candidate evaluation. The donor candidate should know that their personal health information will only be disclosed to their intended recipient or other parties if they provide permission to do so. The donor candidate should also know that it is likely they will be asked for permission to disclose certain personal health information to their intended recipient so that the intended recipient can provide informed consent for the transplant to occur. ${ }^{69,70}$ For example, in directed donation, while a donor candidate may wish to keep their act of donor evaluation initially confidential, if the transplant program does not permit anonymous directed donation, or if the intended recipient does not wish to proceed with anonymous directed donation, there may be a need for the donor candidate to provide permission for their identity to be disclosed to the intended recipient so that the intended recipient can make an informed decision about proceeding with the transplant. Similarly, donor candidates and intended recipients need to provide permission to make each other aware whether they are biologically compatible or not. During the donor evaluation process, it may be recognized that a donor candidate has additional health information that could impact the transplant outcome. For example, despite negative serological testing, a donor candidate may have a higher risk of specific infectious diseases based on his/her behavioral history. Some jurisdictions require standardized behavioral screening ${ }^{71}$ during the evaluation and consent from the donor candidate to inform the intended recipient of behavior associated with increased risk of certain infections, so that the intended recipient can provide informed consent for the transplant to proceed. ${ }^{51}$ Before donation the transplant program should also inform the donor candidate of requirements to share certain personal health information with the recipient after donation, such as in the rare circumstance where soon after transplant (ie, within 1-2 years) it is discovered the donor has evidence of a serious infectious disease or malignancy. ${ }^{51}$

It is possible that the intended recipient has health information that could impact transplant outcomes, and which the transplant team believes could affect the donor candidate's decision making. For example, some donor candidates may want to know if the recipient lost a previously transplanted kidney due to medication nonadherence. ${ }^{72}$ Knowledge of a genetic kidney disease in an intended recipient may be important for the evaluation and care of a genetically related donor candidate. As for the health information of donor candidates, personal health information collected during the transplant candidate's evaluation is protected under privacy law, and can only be shared with permission of the intended recipient. Prior guidelines, such as those of the British Transplantation Society and policy of the US OPTN, recommend disclosure of relevant information about the intended recipient to the donor candidate if the intended recipient has given consent $^{48,51}$; the British Transplantation Society also recommends that donation and transplant not proceed unless the relevant information is shared. ${ }^{48}$ The criteria for relevant information beyond the determination that the intended recipient is approved as a suitable transplant candidate by their evaluation team are currently undefined. Ongoing efforts are warranted to develop standardized criteria for the identification and disclosure of recipient risk factors for adverse transplant outcomes that may be relevant to donor decision making, and when donation and transplant should not proceed in the absence of disclosure, weighing considerations of privacy law, ethics, and the concerns of donor and recipient candidates (see Research Recommendations).

Donor candidates should be informed of transplant program resources and personnel available to offer support. Such support can include psychological support in the setting of a poor recipient outcome after transplantation or donor complications, or financial reimbursement programs for out-of-pocket expenses incurred during the evaluation and donation process. The donor candidate should understand what is required of them after donation, including the likely timing and financial impacts of donation-related recommended lifelong healthcare. The donor candidate should also understand how this care will be provided after donation, and the transplant program's policy for providing any healthcare (and what types of healthcare) after donation. In the US transplant programs are required to collect and report follow-up data on donors for 2 years after donation. ${ }^{73}$

In nearly all places in the world it is a crime to knowingly acquire or obtain any human organ for valuable consideration (ie, for anything of value such as money or property). Some prior guidelines recommend the donor candidate sign a statement attesting that they are not donating a kidney for monetary gain ${ }^{46,74}$; in the United States, such attestation is required for donation to proceed. ${ }^{51}$ The donor candidate should understand the withdrawal process from evaluation, and their right to withdraw at any time before donation with the full support of the transplant program (described further below in this guideline chapter). Finally, while transplant programs respect the autonomy of a donor candidate to proceed with donation based on their preferences, needs and values, programs remain ethically justified to decline a donor 
candidate who does not meet their eligibility criteria for donation (ie, when the donation is deemed to incur unacceptable risks). ${ }^{22} \mathrm{~A}$ donor candidate should understand the transplant program makes the final determination of whether the donor candidate is eligible for donation or not based on the results of their evaluation. If the donor candidate does not meet the transplant program's criteria for donation, the program should inform the donor candidate of the decision and reason. Being told they do not meet a transplant program's acceptance criteria is distressing for some donor candidates. $^{75}$ The donor candidate should be informed how the transplant program will support them if they do not meet criteria for donation. Such support may include assistance in communicating the decision to the intended recipient; ongoing follow-up communication with the evaluation team; counseling related to the outcome of the evaluation; alternative ways of helping the intended recipient; and the possibility of referral to another program for a second opinion if the donor candidate does not accept the noneligibility decision.

\section{Comprehension of Disclosed Information}

Donor candidates should have adequate time to consider the information they are provided during the evaluation process, as this is required for informed consent. The duration of adequate time is not well defined, and may vary according to donor characteristics. Some, but not all transplant programs, require all donor candidates to exercise a minimal period for this adequate consideration, referred to as a 'cooling-off' period. ${ }^{44}$

In current routine care, an assessment of a donor candidate's knowledge and comprehension of the possible outcomes of donation is typically done through discussions with healthcare professionals. Optimal methods to assess understanding in living donor candidates are not well defined, ${ }^{39,58}$ but general techniques for comprehension assessment may include use of "teach-back," in which patients are asked to "teach back" what they have learned during their visit. ${ }^{76}$ An instrument for assessing comprehension during informed consent for living liver donation has been pilot tested, ${ }^{77}$ and provides a model for developing similar instruments for comprehension assessment in living kidney donor candidates. It is common for many donor candidates to voice no concerns during the evaluation process about the donation, as they are using an emotional rather than deliberative decision-making process. Some donors have an exaggerated sense of the true benefit of donation to their intended recipient, while others underappreciate the amount of postoperative physical pain they may experience or the time needed to fully recover after surgery. ${ }^{78}$

\section{The Voluntary Nature of Kidney Donation}

Voluntarism is established when a transplant program ensures the donor candidate is free from undue pressure or coercion in deciding whether or not to donate. ${ }^{79}$ Voluntarism should be respected by all members of the transplant team; as discussed above, additional safeguards may include use of Independent Living Donor Advocates or external reviews of planned donations. ${ }^{51,52}$ Special groups such as prisoners have unique considerations. ${ }^{80}$ Interviewing the donor candidate without the intended recipient, family members and other persons who could influence the donation decision is important in the assessment of voluntarism. Trust is maintained when the transplant program assures a donor candidate that their participation in evaluation and personal health information is confidential, to be shared with the intended recipient only with their approval. This enables the donor candidate to speak openly about their health and donation choices.

For the purposes of donor candidacy, 'not deciding' about donation should be considered the same as 'deciding not to' proceed, as may occur in cases of ambivalent donor candidates who have not decided to proceed, but who also have not elected to formally withdraw from the donation process. ${ }^{39}$

Transplant programs should support donor candidates who decide to withdraw from the evaluation process or decline to donate in a way that is respectful and confidential. ${ }^{81}$ So-called 'medical alibis,' provision of a false medical reason to justify unsuitability as a living donor, are discouraged as untruthful statements may undermine trust in the transplant program and the patient/physician relationship. ${ }^{81}$ Thiessen et al recommend that all donor candidates be offered a general statement regarding 'unsuitability to donate' at any time; there is controversy about whether the transplant program should assist the donor candidate with wording that includes factual medical findings which may or may not preclude donation (such as mildly elevated BP or risk factors for metabolic syndrome). ${ }^{82}$ Understandably, a donor candidate who decides not to donate may have difficulty communicating this decision to the intended recipient or others; in such circumstances the transplant program should assist with this communication, which may involve communication through the recipient evaluation team to the transplant candidate.

\section{RESEARCH RECOMMENDATIONS}

- Determine the best methods to achieve informed consent from living kidney donor candidates, including what methods are most useful to impart information including risks and outcomes and what methods are most useful to assess comprehension. ${ }^{42}$

- Through focus groups and/or surveys, develop standardized criteria for circumstances under which intended recipients should be asked for permission to disclose certain personal health information to the living kidney donor candidate (such as loss of a prior graft due to medication nonadherence), so that donor candidates can make an informed decision about whether to proceed with donation or not.

- Develop standardized criteria for when donation and transplant should not proceed in the absence of disclosure, weighing considerations of privacy law, ethics, and the concerns of donor and recipient candidates.

- Perform postdonation surveys to measure donors' assessments of the quality of standardized informed consent processes, including if the information provided before donation met the donor's needs and prepared them for the donation. ${ }^{83}$

- Compare experiences of donors who donated before and after the implementation of country-specific regulations for better informed consent processes for the practice of living donation.

- Evaluate appropriate circumstances for and approaches to substitute decision making and use of surrogate consent, including definition of the necessary supporting ethical framework for particular scenarios.

- Develop ethically-grounded, practical strategies to consider and manage evaluation of living kidney donor candidates identified by the intended recipient or their representatives through mass advertising and social media. ${ }^{84}$ 


\section{CHAPTER 3: COMPATIBILITY TESTING, INCOMPATIBLE TRANSPLANTATION, AND PAIRED DONATION}

The ERT search parameters did not identify evidence from eligible studies pertinent to the recommendations in chapter 3 and therefore the following recommendations are "Not Graded.”

\section{Evaluation}

3.1: Donor ABO blood typing should be performed twice before donation to reduce the risk of unintended blood type incompatible transplantation.

3.2: Donor blood group A subtype testing should be performed when donation is planned to recipients with anti-A antibodies.

3.3: Human leukocyte antigen (HLA) typing for major histocompatibility complex (MHC) Class I (A, B, C) and Class II (DP, DQ, DR) should be performed in donor candidates and their intended recipients, and donor-specific anti-HLA antibodies should be assessed in intended recipients.

\section{Counseling}

3.4: Donor candidates who are ABO blood group or HLA incompatible with their intended recipient should be informed of availability, risks, and benefits of treatment options, including kidney paired donation and incompatibility management strategies.

3.5: If a donor candidate and their intended recipient are blood type or crossmatch incompatible, transplantation should be performed only with an effective incompatibility management strategy.

3.6: Nondirected donor candidates should be informed of availability, risks and benefits of participating in kidney paired donation.

\section{RATIONALE}

\section{Evaluation: Blood Type and Histocompatibility Testing}

ABO blood typing should be performed in living donor candidates before donation, including routine duplicate testing, to reduce the risk of unintended blood type-incompatible transplantation. Unintended ABO-incompatible (ABOi) transplantation should be avoidable with $\mathrm{ABO}$ typing of the donor and the recipient; however, human errors have led to cases of accidental ABOi organ transplantation in contemporary practice. ${ }^{85} \mathrm{ABO}$-subtype testing should be performed when donation is planned to recipients with anti-A antibodies. ${ }^{86}$ Human leukocyte antigen (HLA) typing for major histocompatibility complex (MHC) class I (A, B, C) and class II (DP, DQ, DR) should be obtained in living donor candidates for recipient candidates with anti-HLA antibodies, as part of the assessment of compatibility during preoperative planning; there is an association between the presence of HLA-C and/or HLA-DP and DQ and a higher incidence of graft rejection. ${ }^{87,88}$ While recipient care is out of the scope of this guideline, it is important to emphasize that recipient candidates should undergo antidonor antibody examinations, including complement-dependent cytotoxicity or flow cytometry crossmatching and Luminex (Bio-Rad Laboratories, Inc., Hercules, CA) assays to determine the history of sensitization, ${ }^{89}$ and this testing should be current before proceeding with donor nephrectomy and living donor transplantation.

\section{Counseling Regarding Transplant Options and Expected Outcomes}

Biological incompatibility remains a significant barrier to living donor kidney transplantation. Estimates based on blood group prevalence in the United States suggest that more than $35 \%$ of willing, healthy potential living donors are blood group incompatible with their intended recipients. ${ }^{90}$ Options for transplant candidates whose only willing, healthy donor is ABO or HLA incompatible include: KPD, planned incompatible transplantation (with preconditioning/desensitization treatment of the intended recipient as needed), attempting to find a different living donor, or waiting for a compatible deceased donor organ. ${ }^{91-93}$ While evaluation and management of the transplant recipient is not within the scope of this project, outcomes of recipients after various forms of transplant or waiting are relevant to living kidney donor candidate education. Perspectives of risk and benefit for counseling of the intended recipient and donor candidate include outcomes after compatible versus incompatible transplantation, outcomes after incompatible transplantation versus dialysis, and the likelihood of transplantation with options including KPD programs. Donor candidates who are ABO or HLA incompatible with their intended recipient should be informed of expected patient and graft survival for KPD and incompatible transplantation, as compared with compatible living donor transplantation and deceased donor transplantation, as well as expected patient survival on dialysis, based on best available information.

\section{Kidney Paired Donation}

KPD has emerged as a successful approach to address $\mathrm{ABO}$ blood group and HLA incompatibilities for those who have a willing, but incompatible living donor candidate. The fastest growing modality for living donor transplantation is KPD, rising from 2 cases in 2000 to approximately 700 cases reported to the US OPTN in $2013 .{ }^{94}$ In 2004, the Netherlands instituted a paired exchange system in all their transplant centers, which may explain the recent increase in living kidney donation in that country. ${ }^{95}$

Donor candidates who are ABO or HLA incompatible with their intended recipient should be informed of the availability of $\mathrm{KPD}$, and the considerations related to pursuit of this treatment option. Participation in KPD requires KPD program-specific informed consent ${ }^{60,61}$ (Please see also chapter 2: Informed Consent for details). The transplant program should inform the donor candidate of policies regarding confidentiality and anonymity in KPD and nondirected donation, and should ensure donor acceptance of these policies before donation. ${ }^{62}$ Some programs now permit biologically compatible pairs to participate in KPD; when this option is discussed, donor candidates who are biologically compatible with their intended recipient should voluntarily decide whether or not to pursue this option. ${ }^{63-65}$

Donor candidates participating in KPD should be informed of the risks and benefits of kidney transport, possible organ redirection due to unforeseen circumstances, the inability to provide information on the ultimate recipient of their organ, as well as nonexchange donation options. ${ }^{60} \mathrm{Liv}$ ing donors participating in exchanges should have the option to travel to the recipient center; however, experience from countries with well-developed KPD programs suggests that 
transport of living donor kidneys can be accomplished safely without adversely impacting transplant outcomes, obviating the need for donor travel. In a survey of 30 US programs transporting 56 living donor kidneys (2007-2010), the creatinine nadir was less than $2.0 \mathrm{mg} / \mathrm{dL}(<177 \mu \mathrm{mol} / \mathrm{L})$ in all recipients but one, and there were no cases of delayed graft function as defined by the need for dialysis in the first week. ${ }^{96}$ Continued feasible and safe transport of living donor kidneys in the United States has been reported. ${ }^{97}$

Nondirected donors (donor without an identified recipient) have the unique potential to expand the donor pool through chains of kidney exchanges. ${ }^{98}$ Nondirected donor candidates should be informed of opportunities for donating into a chain or KPD program, if available.

Participation in KPD should be considered preferable to incompatible transplantation if participation is deemed to have "reasonable" likelihood of yielding a compatible or lower-immunologic risk match for the donor candidate's intended recipient. Despite the expansion of KPD, blood group $\mathrm{O}$ candidates continue to have much lower rates of success on KPD lists than their non-O counterparts, particularly in circumstances of broad HLA sensitization. ${ }^{86}$ Thus, for some transplant candidates, incompatible transplantation may offer their best option for transplantation without prolonged waiting times.

\section{Blood Type-Incompatible Living Donor Transplantation}

While incompatible transplantation without preconditioning/ desensitization treatment of the recipient may lead to hyperacute rejection and allograft loss, predetermined incompatibility management protocols have been developed in recent decades. In 1987, successful ABOi living donor transplantation was introduced in Japan using pretransplant antibody depletion, to expand access to transplantation in the absence of legal recognition of brain death. ${ }^{99-101}$ Since that time, ABOi transplantation evolved into routine practice and constituted nearly $14 \%$ of living donor transplant procedures performed in Japan in 2011.

Recipient and donor candidates interested in ABOi living donor transplantation should be informed of possible complications and expected outcomes. US studies have reported higher rates of perioperative complications including hemorrhage, infections, and early graft loss compared with $\mathrm{ABO}$ compatible (ABOc) transplantation. ${ }^{91,102}$ In contrast, some European and Asian studies have found no increases in early or longer-term complications after ABOi transplantation, ${ }^{103-106}$ possibly reflecting differences in preconditioning management protocols. Even in the US experience, after an early reduction in graft survival relative to blood ABOc living donor kidney transplant recipients, ${ }^{91}$ the average long-term graft survival in ABOi living donor transplant recipients is not inferior to, and often exceeds, that of $\mathrm{ABOc}$ deceased donor transplant recipients. ${ }^{91,107}$ In the United States, recipients of ABOi living donor kidney transplants also appear to incur higher costs of care before, during, and after transplant, although these costs increases are offset by avoiding longterm dialysis and its associated morbidity and costs. ${ }^{108}$

\section{HLA-Incompatible Living Donor Transplantation}

HLA-incompatible transplantation remains the most difficult hurdle in achieving successful transplant outcomes. Flow cytometry crossmatching + or Luminex +/complement dependent cytotoxicity- incompatibility may be acceptable with management including B-cell-depleting treatments (eg, anti-CD 20 antibody, rituximab) and/or splenectomy and/or intravenous immunoglobulin, but increased risk of early rejection remains, requiring additional immunosuppression and attendant risks to the recipient. Nonetheless, while allograft survival after HLA-incompatible living donor kidney transplantation is inferior to compatible transplantation, incompatible transplantation after desensitization may offer a substantial survival benefit compared with dialysis or waiting for a deceased donor kidney; however, there are few high-quality studies testing this hypothesis. A recent study compared 1025 recipients of HLA-incompatible living donor kidney transplants at 22 US medical centers with matched controls who remained on waiting lists or waited and received a transplant from a compatible deceased donor. After 8 years, $76.5 \%$ of those who received an incompatible living donor transplant were still alive, compared with $62.9 \%$ who remained on the waiting list or received a deceased donor transplant and only $43.9 \%$ who remained on the waiting list but were never transplanted. ${ }^{109}$ HLA-incompatible transplantation does confer additional costs compared with compatible transplantation, but may be cost-effective compared with dialysis; formal cost effectiveness evaluations are needed. ${ }^{110}$

\section{What Prior Guidelines Recommend}

In contrast with our recommendations, the European Renal Best Practice (ERBP) guideline for kidney donor and recipient evaluation recommends performing HLA-DQ, HLA-DP and HLA-C typing of the donor only when the intended recipient has HLA antibodies against those antigens. ${ }^{50}$ The ERBP does not recommend routine typing for MHC Class I-related chain-A and other non-HLA antigens in either recipient or donor. Similar to our recommendations, the ERBP recommends establishing programs to select a donor towards whom the recipient does not produce antibodies through KPD, and recommends transplanting patients with donor-specific antibodies only if this cannot be accomplished by KPD. ${ }^{50}$ Details of testing and clinical management associated with HLA and non-HLA antibodies in transplantation are out of the scope of the current guideline, but consensus-based recommendations from a 2012 Transplantation Society work group are available. ${ }^{89}$

In 2012, the American Society of Transplantation (AST)/ American Society of Transplant Surgeons (ASTS) held a consensus conference directed at overcoming barriers to the adoption of KPD that produced recommendations related to KPD donor evaluation and care, guidelines for KPD histocompatibility testing, and recommended policies to overcoming geographic barriers to KPD. ${ }^{60}$

\section{RESEARCH RECOMMENDATIONS}

- Define mediators of clinical outcomes and optimal management of ABO and HLA incompatible living donor transplantation to support donor and recipient selection, understanding of transplant utility and informed consent, including:

- Standardized, controlled comparisons of preconditioning/ desensitization and posttransplant immunosuppressive protocols for incompatible transplantation

Elucidation of the mechanisms of antibody production and long-term impact on the allograft

- Develop a long-term, appropriately powered RCT to compare the outcomes and cost effectiveness of options for highly sensitized candidates including participation in KPD with varying 
waiting times versus living donor transplantation after various desensitization protocols.

\section{CHAPTER 4: PREOPERATIVE EVALUATION AND MANAGEMENT}

The ERT search parameters did not identify evidence from eligible studies pertinent to the recommendations in chapter 4 and therefore the following recommendations are "Not Graded.”

4.1: Donor candidates should receive guideline-based evaluation and management used for other noncardiac surgeries to minimize risks of perioperative complications, including a detailed history and examination to assess risks for cardiac, pulmonary, bleeding, anesthesia-related and other perioperative complications.

4.2: Donor candidates who smoke should be advised to quit at least 4 weeks before donation to reduce their risk of perioperative complications, and commit to lifelong abstinence to prevent long-term complications.

\section{RATIONALE}

\section{Evaluation}

The goals of the general preoperative evaluation are to assess a donor candidate's risk of perioperative complications according to their profile of predonation characteristics assessed through a careful medical history, physical examination and testing; to determine if this risk is acceptable to proceed with donation; and to counsel the donor candidate on how to minimize their risk of perioperative complications (eg, stop smoking, lose weight if obese). The donor then receives care during the perioperative period to minimize their risk of complications, so that they can return to their level of presurgical function as quickly as possible. Recommendations on how to achieve these outcomes with good preoperative evaluation and management are sparse in prior living kidney donor guidelines, other than a description of elements of the detailed history required before donation (eg, prior surgeries, anesthesia-related reactions and bleeding disorders). ${ }^{51}$ Associations of donation-specific surgical techniques with perioperative outcomes are described elsewhere (see chapter 17).

\section{Risks of Perinephrectomy Complications}

Living kidney donor nephrectomy is an elective surgical procedure that carries a low risk for complications as compared with other types of surgical procedures. As described in chapter 1, the 90-day all-cause mortality in a US study of integrated donor registry data and national death records (80 347 donors, 1994-2009) was approximately 1 in 3000 $(0.03 \%)$ based on 25 deaths. $^{23}$ Similar rates have also been reported in other studies. ${ }^{24,25}$ Given the low incidence of perioperative mortality, estimates for predonation characteristics that alter the risk of perioperative death are imprecise.

A US study recently described the incidence of perioperative complications in a large sample of living kidney donors from 1998 to $2010 .^{28}$ Outcomes were assessed through administrative data, using a composite outcome of digestive, respiratory, procedural, urinary, hemorrhage, infectious or cardiac complications. The incidence of perioperative complications was 7.9\% and decreased from 1998 to 2010 (from $10.1 \%$ to $7.6 \%$ ). Limitations of this study include the lack of confirmation of donor status through patient level-linkages to the national registry, and use of weighting schemes to draw inferences for a "represented" sample of all US donors based on a stratified sample of $20 \%$ of acute care hospitalizations. A subsequent study integrated national US donor registry data as a source of verified living donor status with administrative records from a consortium of 98 academic hospitals (2008 to 2012, $n=14964$ ), and found that $16.8 \%$ of donors experienced any perioperative complication, most commonly gastrointestinal $(4.4 \%)$, bleeding $(3.0 \%)$, respiratory $(2.5 \%)$, and surgical/anesthesia-related injuries $(2.4 \%){ }^{29}$ Major complications, defined as Clavien grading system for surgical complications level 4 or $5,{ }^{111}$ affected $2.5 \%$ of donors. The limitations of administrative database studies, including possible coding biases, highlight the need for prospective collection of granular clinical data on living donor perioperative outcomes. A Norwegian registry-based study of 1022 living kidney donations performed between 1997 and 2008 recorded $30(2.9 \%)$ major complications and $184(18 \%) \mathrm{mi}-$ nor complications by Clavien grading. ${ }^{112}$

Readmission after surgery is commonly used as a measure of care quality and healthcare utilization. A recent study examined postnephrectomy readmission using integrated US living donor registry data, records from a nationwide pharmacy claims warehouse, and administrative records from an academic hospital consortium ( $\mathrm{N}=14959$ donors, 2008-2012). ${ }^{113}$ Overall, $2.9 \%$ of donors were readmitted to hospital within 90 days of donation. $11.3 \%$ donors filled 1 or greater opioid prescription in the year before donation, and those with the highest level predonation opioid use were more than twice as likely as nonusers to be readmitted within 90 days postdonation $(6.8 \%$ vs $2.6 \%$; adjusted odds ratio

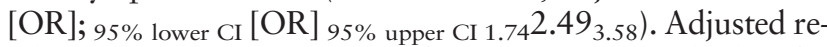
admission risk was also significantly $(P<0.05)$ higher for women (adjusted OR = 1.25), African Americans (adjusted $\mathrm{OR}=1.45$ ), spouses (adjusted OR $=1.42$ ), exchange participants (adjusted OR $=1.46$ ), uninsured donors (adjusted $\mathrm{OR}=1.40$ ), donors with predonation eGFR $<60 \mathrm{~mL} / \mathrm{min}$ per $1.73 \mathrm{~m}^{2}$ (adjusted OR = 2.68), donors with predonation pulmonary disease (adjusted OR $=1.54$ ), and after robotic nephrectomy (adjusted OR $=1.68$ ). Continued efforts to identify and prevent modifiable causes of postoperative complications in all donors are warranted to maximize safety of the donation procedure.

Readers are encouraged to refer to chapter 17 for discussion of acceptable surgical approaches to donor nephrectomy and anticipated outcomes. As discussed in the Framework for this guideline (chapter 1), the transplant program team should provide the donor candidate with individualized quantitative estimates of short-term and long-term risks from kidney donation to the extent possible, including recognition of associated uncertainty, in a manner that is easily understood by donor candidates.

\section{Preoperative and Perioperative Management to Minimize the Risk of Perinephrectomy Complications}

Guidelines for evaluation and management before noncardiac surgery in the general population have been thoroughly reviewed and summarized. ${ }^{114}$ There is no evidence to suggest that additional preoperative testing beyond guidelinebased evaluation and management strategies used for other noncardiac surgeries results in a reduced incidence of perioperative complications in kidney donors. 
Some transplant programs routinely perform preoperative noninvasive cardiac testing in older living kidney donor candidates (eg, stress electrocardiogram, nuclear stress test), but this practice is not supported by existing evidence. Recent US guidelines for the general population do not recommend cardiac testing for those undergoing noncardiac surgery with no active symptoms of heart disease who have reasonable functional capacity (defined as at least 4 metabolic energy equivalents, which represents the ability to walk 2 blocks on ground level or carry 2 bags of groceries up one flight of stairs without symptoms). ${ }^{115}$ Outside the context of perioperative evaluation, other guidelines recommended against noninvasive cardiac testing for asymptomatic coronary artery disease, or conclude that there is insufficient evidence to warrant such testing. ${ }^{116}$

Recent guidelines on the assessment of bleeding risk before surgery or invasive procedures describe the following ${ }^{117}$ : (i) Routine coagulation screening before surgery to predict postoperative bleeding in unselected patients is not recommended; (ii) bleeding history assessment should include details of a family history of bleeding, previous excessive posttraumatic or postsurgical bleeding, and current use of antithrombotic drugs; (iii) if the bleeding history is negative, no further coagulation testing is indicated; (iv) comprehensive coagulation testing is warranted if the bleeding history is positive. Although evidence in the context of donor nephrectomy is lacking, current OPTN policy for living donor evaluation in the United States requires assessment of bleeding and clotting disorders in the medical history, and performance of coagulation testing, ${ }^{51}$ and other prior living donor evaluation guidelines have recommended blood coagulation testing. ${ }^{54,118}$

In a recent multicenter randomized trial of 10010 patients undergoing noncardiac surgery, the use of aspirin before surgery and throughout the early postsurgical period had no significant effect on the risk of death or nonfatal myocardial infarction but increased the risk of major bleeding. ${ }^{119}$ The trial authors recommend aspirin not be started before surgery, and for those chronically taking aspirin to hold it at least 3 days before surgery.

Recent guidelines for the prevention of perioperative venous thromboembolism (ie, deep vein thrombosis and pulmonary embolism) describe the use of a risk score (Rogers or Caprini score) to determine which of early ambulation, mechanical prophylaxis or perioperative unfractionated heparin (or low-molecular-weight heparin) is warranted to reduce risk. ${ }^{120}$ Factors associated with a higher risk of perioperative venous thromboembolism include older age, obesity, and the use of oral contraceptive or hormone replacement therapy. Many donors will be at low risk $(<2 \%)$ of perioperative venous thromboembolism, and some guidelines suggest early ambulation is all that is required in individuals at low risk of such events.

Guidelines to reduce the risk of perioperative pulmonary complications recommend a careful assessment of risk factors for postoperative pulmonary complications (conditions such as chronic obstructive pulmonary disease or congestive heart failure which will be absent in almost all donors). ${ }^{121}$ Preoperative spirometry and chest radiography are not recommended as routine tests to predict the risk for postoperative pulmonary complications. Patients at higher risk for postoperative pulmonary complications may benefit from deep breathing exercises or incentive spirometry, or selective use of a nasogastric tube (as needed for postoperative nausea or vomiting, inability to tolerate oral intake, or symptomatic abdominal distention). The utility and difficulties of the preoperative evaluation for the potential identification of obstructive sleep apnea is described elsewhere. ${ }^{122}$

There have been 6 RCTs that enrolled smokers (ranging from 47 to 213 patients) to receive a smoking cessation intervention or not before surgery (procedures other than donor nephrectomy $)^{123}$ and found that smoking cessation reduced the risk of perioperative complications.

\section{RESEARCH RECOMMENDATIONS}

- Develop contemporary estimates of 5.10, the risk of perinephrectomy complications according to an individualized profile of predonation characteristics, accounting for changes in baseline comorbidity burdens.

- Assess the predictive value of novel risk factors for perioperative complications and readmission after donor nephrectomy, including use of opioids and other pharmaceuticals. ${ }^{113}$

- Perform RCTs to formally assess the efficacy of evaluation and perioperative management techniques to minimize the risk of perioperative complications after living donor nephrectomy.

\section{CHAPTER 5: PREDONATION KIDNEY FUNCTION}

Except in the case of Recommendation 5.10, the ERT search parameters did not identify evidence from eligible studies pertinent to the recommendations in chapter 5 and therefore the following recommendations are "Not Graded." Some of the recommendations extrapolated from the 2012 KDIGO CKD Guideline (Section 1.4.3) ${ }^{124}$ were not part of the ERT review for this guideline and as such they are also "Not Graded."

\section{Evaluation}

5.1: Donor kidney function should be expressed as glomerular filtration rate (GFR) and not as serum creatinine concentration.

5.2: Donor GFR should be expressed in $\mathrm{mL} / \mathrm{min}$ per $1.73 \mathrm{~m}^{2}$ rather than $\mathrm{mL} / \mathrm{min}$.

5.3: Donor glomerular filtration rate (GFR) should be estimated from serum creatinine $\left(\mathrm{eGFR}_{\mathrm{cr}}\right)$ for initial assessment, following recommendations from the KDIGO 2012 CKD guideline.

5.4: Donor GFR should be confirmed using one or more of the following measurements, depending on availability:

- Measured GFR (mGFR) using an exogenous filtration marker, preferably urinary or plasma clearance of inulin, urinary or plasma clearance of iothalamate, urinary or plasma clearance of ${ }^{51} \mathrm{Cr}$-EDTA, urinary or plasma clearance of iohexol, or urinary clearance of ${ }^{99 \mathrm{~m}} \mathrm{Tc}$-DTPA

- Measured creatinine clearance $(\mathrm{mCrCl})$

- Estimated GFR from the combination of serum creatinine and cystatin $\mathrm{C}\left(\mathrm{eGFR}_{\text {cr-cys }}\right)$ following recommendations from the KDIGO 2012 CKD guideline

- Repeat estimated GFR from serum creatinine $\left(\mathrm{eGFR}_{\mathrm{cr}}\right)$

5.5: If there are parenchymal, vascular or urological abnormalities or asymmetry of kidney size on renal imaging, single kidney GFR should be assessed using radionuclides or contrast agents that are excreted by glomerular filtration (eg, $\left.{ }^{99 \mathrm{~m}} \mathrm{Tc}-\mathrm{DTPA}\right)$. 


\section{Selection}

5.6: GFR of $90 \mathrm{~mL} / \mathrm{min}$ per $1.73 \mathrm{~m}^{2}$ or greater should be considered an acceptable level of kidney function for donation.

5.7: The decision to approve donor candidates with GFR 60 to $89 \mathrm{~mL} / \mathrm{min}$ per $1.73 \mathrm{~m}^{2}$ should be individualized based on demographic and health profile in relation to the transplant program's acceptable risk threshold.

5.8: Donor candidates with GFR less than $60 \mathrm{~mL} / \mathrm{min}$ per $1.73 \mathrm{~m}^{2}$ should not donate.

5.9: When asymmetry in GFR, parenchymal abnormalities, vascular abnormalities, or urological abnormalities are present but do not preclude donation, the more severely affected kidney should be used for donation.

\section{Counseling}

5.10: We suggest that donor candidates be informed that the future risk of developing kidney failure necessitating treatment with dialysis or transplantation is slightly higher because of donation; however, average absolute risk in the 15 years following donation remains low. (2C)

\section{RATIONALE}

\section{Evaluation}

The goals of the evaluation of kidney function in living donor candidates are to:

- Provide accurate assessment of level of GFR and prediction of long-term risk of ESKD (in the absence of and after donation) based on level of predonation GFR and other factors.

- Allow identification and exclusion of donor candidates whose postdonation risks are expected to exceed the acceptable risk threshold established by the transplant program.

- Provide counseling regarding level of risk for donor candidates whose long-term risks for ESKD are expected to be below the acceptable risk established by the transplant program.

- Provide counseling regarding the need for follow-up of decreased GFR after donation.

For this section, recommendations related to measurement of kidney function are based on physiological principles and recommendations for general clinical practice from the KDIGO 2012 CKD guideline. ${ }^{124}$ These recommendations were based on a systematic review of the literature, which included some studies of donors before and after kidney donation. ${ }^{125}$ There is no evidence to suggest that living kidney donor candidates or kidney donors differ from other populations regarding these recommendations.

\section{Box 1. Key recommendations from the KDIGO 2012 CKD guideline regarding GFR estimation. ${ }^{124}$}

- We recommend using serum creatinine and a GFR estimating equation for initial assessment (Recommendation 1.4.3.1, Grade 1A).

- We suggest using additional tests (such as cystatin $\mathrm{C}$ or a clearance measurement) for confirmatory testing in specific circumstances when eGFR based on serum creatinine is less accurate (Recommendation 1.4.3.2: Grade 2B).
- We recommend that clinicians (Recommendation 1.4.3.3, Grade 1B):

- Use a GFR estimating equation to derive GFR from serum creatinine $\left(e G F R_{c r}\right)$ rather than relying on the serum creatinine concentration alone.

- Understand clinical settings in which $\mathrm{eGFR}_{\mathrm{cr}}$ is less accurate.

- We recommend that clinical laboratories should (Recommendation 1.4.3.4, Grade 1B):

- Measure serum creatinine using a specific assay with calibration traceable to the international standard reference materials and minimal bias compared to isotope-dilution mass spectrometry (IDMS) reference methodology.

- Report eGFR cr in addition to the serum creatinine concentration in adults and specify the equation used whenever reporting eGFR $\mathrm{cr}_{\text {. }}$

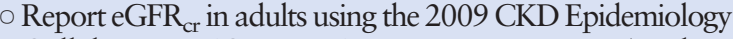
Collaboration (CKD-EPI) creatinine equation. An alternative creatinine-based GFR estimating equation is acceptable if it has been shown to improve accuracy of GFR estimates compared to the 2009 CKD-EPI creatinine equation.

- When reporting serum creatinine:

o We recommend that serum creatinine concentration be reported and rounded to the nearest whole number when expressed as standard international units $(\mu \mathrm{mol} / \mathrm{L})$ and rounded to the nearest 100th of a whole number when expressed as conventional units (mg/dL).

- When reporting eGFR cr $_{\text {: }}$

- We recommend that $\mathrm{eGFR}_{\mathrm{cr}}$ should be reported and rounded to the nearest whole number and relative to a body surface area of $1.73 \mathrm{~m}^{2}$ in adults using the units $\mathrm{mL} / \mathrm{min}$ per $1.73 \mathrm{~m}^{2}$.

o We recommend eGFR cr levels less than $60 \mathrm{~mL} / \mathrm{min}$ per $1.73 \mathrm{~m}^{2}$ should be reported as "decreased."

- If cystatin $\mathrm{C}$ is measured, we suggest that health professionals (Recommendation 1.4.3.6, Grade 2C):

- Use a GFR estimating equation to derive GFR from serum cystatin $\mathrm{C}$ rather than relying on the serum cystatin $\mathrm{C}$ concentration alone.

- Understand clinical settings in which $\mathrm{eGFR}_{\text {cys }}$ and $\mathrm{eGFR}_{\text {cr-cys }}$ are less accurate.

- We recommend that clinical laboratories that measure cystatin C should (Recommendation 1.4.3.7, Grade 1B): - Measure serum cystatin $\mathrm{C}$ using an assay with calibration traceable to the international standard reference material.

- Report eGFR from serum cystatin $\mathrm{C}$ in addition to the serum cystatin $\mathrm{C}$ concentration in adults and specify the equation used whenever reporting eGFR $_{\text {cys }}$ and eGFR cr-cys. $_{\text {. }}$

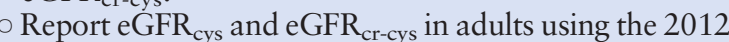
CKD-EPI cystatin C and 2012 CKD-EPI creatininecystatin C equations, respectively, or alternative cystatin C-based GFR estimating equations if they have been shown to improve accuracy of GFR estimates compared to the 2012 CKD-EPI cystatin C and 2012 CKD-EPI creatinine-cystatin $\mathrm{C}$ equations.

- When reporting serum cystatin C:

- We recommend reporting serum cystatin C concentration rounded to the nearest 100 th of a whole number when expressed as conventional units $(\mathrm{mg} / \mathrm{L})$.

- When reporting eGFR $\mathrm{Cys}_{\text {c }}$ and $\mathrm{GGFR}_{\text {cr-cys: }}$ :

- We recommend that $\mathrm{eGFR}_{\text {cys }}$ and $\mathrm{eGFR}_{\text {cr-cys }}$ be reported and rounded to the nearest whole number and 
relative to a body surface area of $1.73 \mathrm{~m}^{2}$ in adults using the units $\mathrm{mL} / \mathrm{min}$ per $1.73 \mathrm{~m}^{2}$.

We recommend eGFR $\mathrm{Cys}_{\text {cys }}$ and eGFR $\mathrm{Gr}_{\text {-cys }}$ levels less than $60 \mathrm{~mL} / \mathrm{min}$ per $1.73 \mathrm{~m}^{2}$ should be reported as "decreased."

- We suggest measuring GFR using an exogenous filtration marker under circumstances where more accurate ascertainment of GFR will impact on treatment decisions (Recommendation 1.4.3.8, Grade 2B).

\section{GFR as an Index of Kidney Function}

The level of GFR is widely accepted as the best overall index of kidney function in health and disease. The foundations for this acceptance are the well-known relationships of alterations in kidney structure and GFR in kidney disease, and well known pathophysiologic relationships of kidney disease complications to decreased GFR. Normative levels of GFR are expressed per $1.73 \mathrm{~m}^{2}$ because GFR is proportional to kidney size, which is proportional to body size. Adjusting GFR to body surface area (BSA) reduces the variability in GFR in healthy individuals, allowing communication of GFR threshold for decision-making that can applied to most donors across the usual distribution of body size.

Based on a large body of evidence, mean GFR in healthy young adult white individuals is approximately $125 \mathrm{~mL} / \mathrm{min}$ per $1.73 \mathrm{~m}^{2}$, with a wide range. ${ }^{126}$ There is some evidence that the normal level of GFR varies among ethnic groups. ${ }^{127}$ GFR is affected by numerous physiologic and pathologic conditions and varies with time of day, dietary protein intake, exercise, age, pregnancy, obesity, hyperglycemia, use of antihypertensive drugs, surfeit or deficit of extracellular fluid, and acute and chronic kidney disease. Despite these limitations, no other measure has been proposed as an overall index of kidney function in the general population or in kidney donor candidates. Thus, it is important that the evaluation of kidney function in kidney donor candidates take into account factors that can affect GFR.

\section{Measurement Methods}

It is not possible to directly measure GFR in humans; thus, the "true" GFR cannot be known with certainty. GFR can be measured indirectly as the clearance of exogenous filtration markers or estimated from serum levels of endogenous filtration markers, but both measured GFR (mGFR) and estimated GFR (eGFR) are associated with error in their determination. We recommend that the transplant program use the best available method to assess GFR in donor candidates, recognizing that at many centers, more than one method may be available. The accuracy of various methods for measuring and estimating GFR is not known with sufficient certainty to define specific threshold for each method.

The KDIGO 2012 CKD guidelines for GFR evaluation in the general population recommend expressing kidney function as GFR and not as serum creatinine concentration, and recommend expressing GFR in $\mathrm{mL} / \mathrm{min}$ per $1.73 \mathrm{~m}^{2}$ rather than $\mathrm{mL} / \mathrm{min} .{ }^{124}$ The guidelines recommend 2-stage testing (initial testing followed by confirmatory testing as necessary). The WG concluded that these general recommendations were applicable to living kidney donor candidates.
The eGFR based on serum creatinine $\left(\mathrm{eGFR}_{\mathrm{cr}}\right)$ is the recommended initial test. Serum creatinine assays should be traceable to the international reference standard. In North America, Europe, and Australia, the 2009 CKD CKD-EPI creatinine equation should be used unless other equations have been shown to be more accurate. eGFR cr using the 2009 CKD-EPI creatinine equation has minimal bias at normal GFR; however, it is imprecise (Figure 5), thus it is most useful for an initial evaluation. ${ }^{128}$ In regions other than North America, Europe, and Australia, the 2009 CKD-EPI creatinine equation is less accurate. In these regions, other equations are recommended if they are more accurate than the 2009 CKD-EPI creatinine equation.

GFR estimating equations are developed using regression methodologies to relate the mGFR to steady state serum creatinine concentration and a combination of demographic and clinical variables as surrogates of the non-GFR determinants of serum creatinine. By definition, GFR estimates using serum creatinine concentration are more accurate in estimating mGFR than the serum creatinine concentration alone in the study population in which they were developed. Sources of error in GFR estimation from serum creatinine concentration include nonsteady-state conditions, nonGFR determinants of serum creatinine, measurement error at higher GFR, and interferences with the creatinine assays (Table 12). GFR estimates are less precise at higher GFR levels than at lower levels. The clinician should remain aware of caveats for any creatinine-based estimating equation which may influence the accuracy in a given individual.

A variety of confirmatory tests for GFR are available. In the general population, the $2012 \mathrm{KDIGO}$ guideline suggests confirmation of GFR with either GFR estimation using cystatin $\mathrm{C}$ or a clearance measurement in specific circumstances when eGFR based on serum creatinine is less accurate (Box 1, 2012 CKD Recommendation 1.4.3.2). KDIGO further

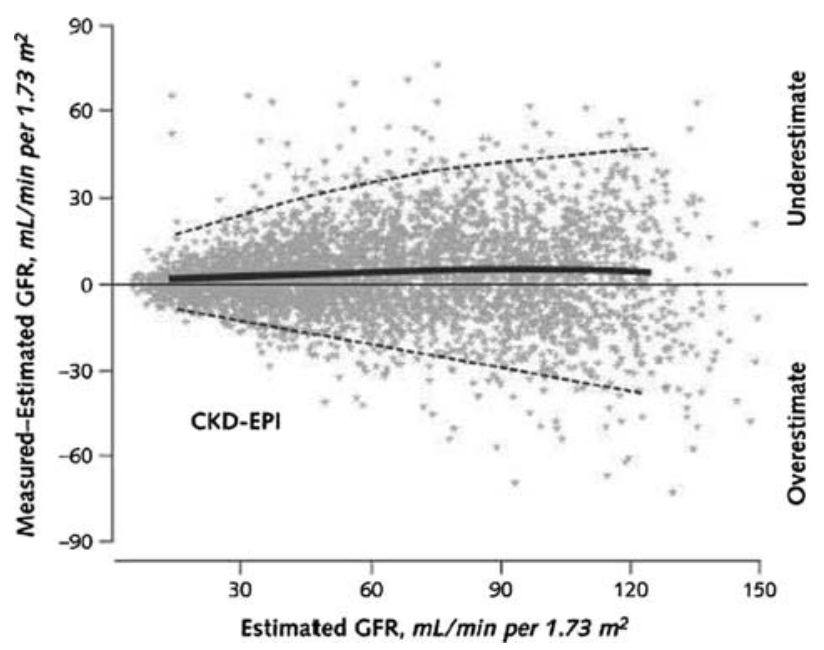

FIGURE 5. Performance of the CKD-EPI equation in estimating measured GFR. The figure shows the difference between measured and estimated GFR (bias) versus estimated GFR in an external validation dataset $(\mathrm{N}=3896)$. A smoothed regression line is shown with the $95 \% \mathrm{Cl}$. Cl, confidence interval; CKD-EPI, Chronic Kidney Disease Epidemiology Collaboration; GFR, glomerular filtration rate. Copyright (C) 2009 American College of Physicians and reprinted with permission from Levey AS, Stevens LA, Schmid $\mathrm{CH}$, et al. A new equation to estimate glomerular filtration rate. Ann Intern Med. 2009;150:604-612. ${ }^{128}$ 
TABLE 12.

Sources of error in GFR estimation using creatinine

\begin{tabular}{|c|c|}
\hline Source of error & Example \\
\hline Non-steady state & AKI \\
\hline \multicolumn{2}{|l|}{$\begin{array}{l}\text { Non-GFR determinants of SCr that differ from study populations } \\
\text { in which equations were developed }\end{array}$} \\
\hline \multirow[t]{8}{*}{ - Factors affecting creatinine generation } & Race/ethnicity other than United States and European black and white \\
\hline & Extremes of muscle mass \\
\hline & Extremes of body size \\
\hline & Diet and nutritional status \\
\hline & - High protein diet \\
\hline & - Creatinine supplements \\
\hline & Muscle wasting diseases \\
\hline & Ingestion of cooked meat \\
\hline \multirow[t]{4}{*}{ - Factors affecting tubular secretion of creatinine } & Decrease by drug-induced inhibition \\
\hline & - Trimethoprim \\
\hline & - Cimetidine \\
\hline & - Fenofibrate \\
\hline \multirow[t]{3}{*}{ - Factors affecting extra-renal elimination of creatinine } & Dialysis \\
\hline & Decrease by inhibition of gut creatininase by antibiotics \\
\hline & Increased by large volume losses of extracellular fluid \\
\hline Higher GFR & $\begin{array}{l}\text { Higher biological variability in non-GFR determinants relative to GFR } \\
\text { • Higher measurement error in SCr and GFR }\end{array}$ \\
\hline \multirow[t]{2}{*}{ Interference with creatinine assay } & Spectral interferences (eg, bilirubin, some drugs) \\
\hline & Chemical interferences (eg, glucose, ketones, bilirubin, some drugs) \\
\hline
\end{tabular}

AKl, acute kidney injury; GFR, glomerular filtration rate; SCr, serum creatinine.

Adapted with permission from Kidney Disease: Improving Global Outcomes (KDIGO) CKD Work Group. KDIGO 2012 clinical practice guideline for the evaluation and management of chronic kidney disease. Kidney Int 2013:1-150. ${ }^{124}$

suggests measuring GFR using an exogenous filtration marker under circumstances where more accurate ascertainment of GFR will impact treatment decisions (Box 1, 2012 CKD Recommendation 1.4.3.8), including the evaluation of living organ donors). Based on these recommendations, the WG concluded that confirmation of GFR using the most accurate method available at the transplant center. In the United States, OPTN policy requires a clearance measurement (measured creatinine clearance $[\mathrm{mCrCl}]$ or $\mathrm{mGFR}$ ) for confirmation of GFR.

Many methods for mGFR determination using exogenous filtration markers and clearance calculations are available, with variable accuracy. Recommendations for specific methods are based on a recent systematic review. ${ }^{129}$ mGFR is not available at all centers, so other alternatives are acceptable. $\mathrm{mCrCl}$ is less accurate than mGFR, ${ }^{129}$ but it is acceptable if $\mathrm{mGFR}$ is not available. $\mathrm{mCrCl}$ overestimates $\mathrm{mGFR}$ due to creatinine secretion. ${ }^{130}$ The magnitude of overestimation is $15 \%$ or more at normal GFR, based on older data using nonstandardized serum creatinine assays. The magnitude of overestimation may be higher using standardized assays. In principle, $\mathrm{mCrCl}$ is available worldwide, however, in some donor candidates, $\mathrm{mCrCl}$ may not be available due to logistical difficulties in collecting or transporting a timed urine collection.

A recent study suggests that eGFR may be sufficiently accurate for decision-making without the need for mGFR in many cases. ${ }^{8}$ A web-based calculator is available to compute posttest probabilities for mGFR above or below threshold probabilities for decision-making: http://ckdepi.org/equations/ donor-candidate-gfr-calculator/. Post-test probabilities are computed from pretest probabilities for mGFR and test performance for eGFR using serum creatinine $\left(e G F R_{c r}\right)$ or the combination of creatinine and cystatin $\mathrm{C}\left(\mathrm{eGFR}_{\mathrm{cr}-\mathrm{cys}}\right)$. Very high posttest probabilities provide reassurance that $\mathrm{mGFR}$ is above the threshold level for decision-making, while very low posttest probabilities provide reassurance that mGFR is below the threshold levels for decision-making. Transplant programs can determine what posttest probabilities are sufficient for clinical decision-making in the absence of $\mathrm{mGFR}$ and $\mathrm{mCrCl}$. One study validating these computations in donor candidates has been reported. ${ }^{131}$ Future studies should address prediction accuracy among racial and ethnic groups for whom the accuracy of eGFR is less certain (eg, nonblack, nonwhite persons).

In general eGFR $\mathrm{cys}_{\mathrm{s}}$ is not more accurate then $\mathrm{eGFR}_{\mathrm{cr}}$; however using 2 filtration markers improves precision of GFR estimates compared with using either marker alone; thus $\mathrm{eGFR}_{\text {cr-cys }}$ is generally recommended over $\mathrm{eGFR}_{\mathrm{cr}}$ or eGFR $_{\text {cys. }}{ }^{132,133}$ Advantages of cystatin C compared with creatinine are that cystatin $\mathrm{C}$ is not affected by muscle mass and current equations do not require specification of race. Therefore, $\mathrm{eGFR}_{\text {cys }}$ maybe more accurate than $\mathrm{eGFR}_{\mathrm{cr}-\mathrm{cys}}$ in people with very large or very small muscle mass, very high or very low meat intake, or race-ethnicity other than black (African American or African European) or white. If cystatin $\mathrm{C}$ is measured, cystatin $\mathrm{C}$ assays should be traceable to international reference standard (which is in the early stages of implementation) and the 2012 CKD-EPI eGFR cys $_{\text {equation or }}$ eGFR $_{\text {cr-cys }}$ equation should be used unless other equations have been shown to be more accurate. If cystatin $C$ is not available, $\mathrm{eGFR}_{\mathrm{cr}}$ can be used for decision-making. As with creatinine, sources of error in GFR estimation from serum cystatin C concentration include non-steady-state conditions, nonGFR determinants of serum cystatin $C$, measurement 
TABLE 13.

Sources of error in GFR estimation using cystatin C

Source of error

Example

Non-steady state

AKI

Non-GFR determinants of SCysC that differ from study populations in which

equations were developed

- Factors affecting cystatin $\mathrm{C}$ generation

Race/ethnicity other than United States and European black and white Disorders of thyroid function

Administration of corticosteroids

Other hypothesized factors based on epidemiologic associations

(diabetes, adiposity)

- Factors affecting tubular reabosrption of cystatin C

- Factors affecting extra-renal elimination of cystatin C

None identified

Higher GFR

Increased by severe decrease in GFR

Higher biological variability in non-GFR determinants relative to GFR

Higher measurement error in SCysC and GFR

Interference with cystatin $\mathrm{C}$ assay

Heterophilic antibodies

AKl, acute kidney injury; GFR, glomerular filtration rate; SCysC, serum cystatin C.

Adapted with permission from Kidney Disease: Improving Global Outcomes (KDIGO) CKD Work Group. KDIGO 2012 clinical practice guideline for the evaluation and management of chronic kidney disease. Kidney Int 2013:1-150. ${ }^{124}$

error at higher GFR, and interferences with the cystatin C assays (Table 13).

Single ("divided" or "split") kidney GFR can be assessed by radionuclide imaging, but is not required in all kidney donor candidates. However, all kidney donor candidates undergo kidney imaging to detect parenchymal, vascular or urologic abnormalities, and asymmetry in kidney size suggests asymmetry in kidney function.

\section{Selection: Criteria for Acceptable Predonation GFR}

\section{GFR in the General Population}

GFR in young men and women of $90 \mathrm{~mL} / \mathrm{min}$ per $1.73 \mathrm{~m}^{2}$ or greater is generally considered normal. ${ }^{124}$ GFR between 60 and $89 \mathrm{~mL} / \mathrm{min}$ per $1.73 \mathrm{~m}^{2}$ is considered to be decreased compared with the usual level for young adults, but does not meet the KDIGO criterion for CKD.

GFR declines with age, although the cause of decline is not known and the rate of decline appears widely variable. Most data are based on cross-sectional studies, in which mean GFR is lower in older populations than in young populations. Other kidney functions are also lower in older populations (eg, renal plasma flow, maximal urinary concentration) and kidney structure is altered in older populations (eg, cortical atrophy, global glomerulosclerosis, nephrosclerosis). There is debate about whether abnormalities in kidney function and structure in older people represents normal aging or disease.

Decreased GFR in the general population is associated with a higher risk of complications of CKD, including ESKD,
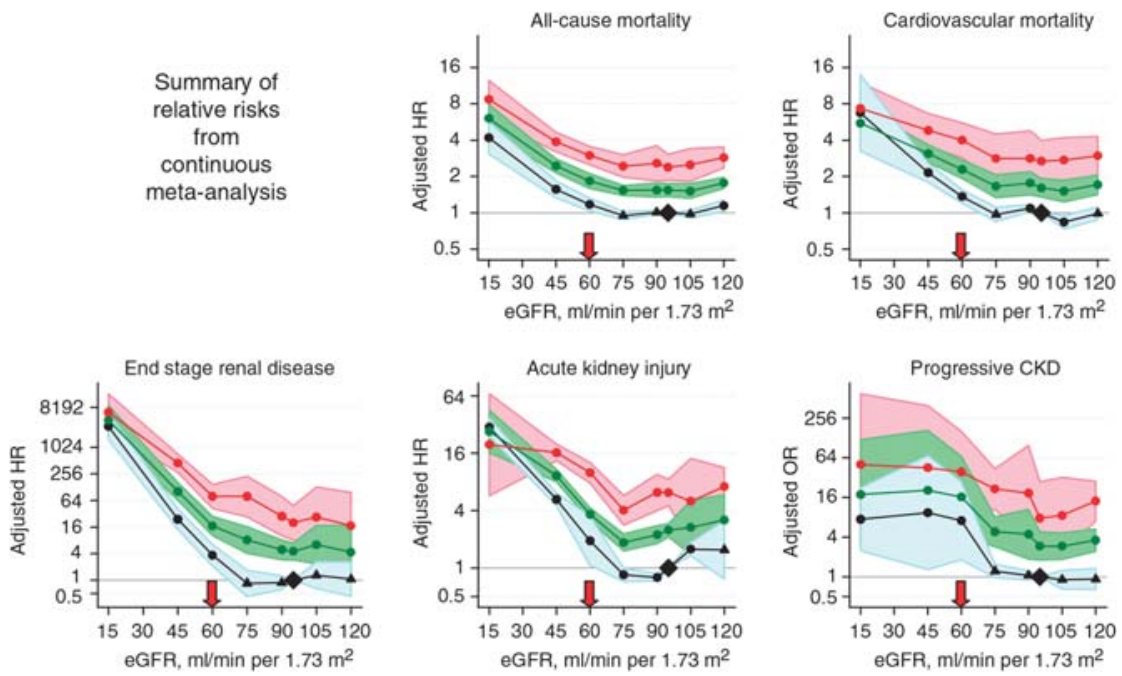

FIGURE 6. Complications of CKD according to baseline eGFR and albuminuria. The data above were derived using creatinine-based eGFR $\left(\mathrm{eGFR}_{\mathrm{cr}}\right.$ ) estimated with the MDRD Study equation. ${ }^{135}$ Comparable data are not available for mGFR. However, studies using more accurate GFR estimates confirm these results. For the outcomes of ESKD, all-cause mortality and cardiovascular mortality, any given reduced level of eGFR is associated with a greater increase in the risk of these outcomes if the $2009 \mathrm{CKD}$-EPI equation is used to estimate eGFR ${ }_{C r}{ }^{136}$ The increase in risk is even greater if cystatin $\mathrm{C}$ is used to estimate GFR, either with or without creatinine (eGFR $\mathrm{Cr}_{\text {cys }}$ or eGFR $\mathrm{cys}_{\mathrm{s}}$ ), compared with eGFR ${ }_{\mathrm{cr}}{ }^{137} \mathrm{The} 3$ lines represent severe, moderate, and normal/mild albuminuria (top to bottom, respectively). CKD, chronic kidney disease; CKD-EPI, Chronic Kidney Disease Epidemiology Collaboration; eGFR, estimated glomerular filtration rate; ESKD, end-stage kidney disease; GFR, glomerular filtration rate. MDRD, Modification of Diet in Renal Disease. Reprinted with permission from Levey AS, de Jong PE, Coresh J, et al. The definition, classification, and prognosis of chronic kidney disease: a KDIGO Controversies Conference report. Kidney Int. 2011;80:17-28. ${ }^{135}$ 
cardiovascular disease (CVD) and death. In general populations, compared with a reference eGFR of $95 \mathrm{~mL} / \mathrm{min}$ per $1.73 \mathrm{~m}^{2}$, the RR for complications related to decreased eGFR is apparent between 60 and $75 \mathrm{~mL} / \mathrm{min}$ per $1.73 \mathrm{~m}^{2}$ and is exponentially higher at lower eGFR. ${ }^{134}$ Similar relationships of lower eGFR with adverse outcomes have been observed in subgroups including patients with known CKD (Figure 6). ${ }^{134-137}$ KDIGO 2012 guideline defines GFR less than $60 \mathrm{~mL} / \mathrm{min}$ per $1.73 \mathrm{~m}^{2}$ for 3 months or more as satisfying the criteria for CKD. GFR 15 to $30 \mathrm{~mL} / \mathrm{min}$ per $1.73 \mathrm{~m}^{2}$ is defined as severely reduced, and GFR less than $15 \mathrm{~mL} / \mathrm{min}$ per $1.73 \mathrm{~m}^{2}$ is defined as kidney failure. However, the association of lower eGFR with a higher risk of adverse outcomes may be related to other conditions that co-occur with low GFR, such as hypertension, diabetes and CVD. Lower GFR in older people is associated with increased risk for CKD outcomes, including ESKD, CVD and death. The RR for these outcomes in older people with lower eGFR compared with the reference eGFR is less than the RR in younger people, however the increment in absolute risk is higher in older people than in younger people. ${ }^{138}$

A recent meta-analysis based on data from nearly 5 million healthy persons identified from 7 general population cohorts who are similar to kidney donor candidates found that lower GFR (in the absence of donation) is associated with an increased risk for ESKD over median cohort follow-up of 4 to 16 years. ${ }^{7}$ After calibration to annual ESKD incidence in the US healthy population, variations in the projected 15 -year and lifetime risks of ESKD based on level of eGFR were generated according to age, sex, and race for healthy persons (assuming systolic BP (SBP) $120 \mathrm{~mm} \mathrm{Hg}$, urine albumin-to-creatinine ratio (ACR) $4 \mathrm{mg} / \mathrm{g}[0.4 \mathrm{mg} / \mathrm{mmol}]$, BMI $26 \mathrm{~kg} / \mathrm{m}^{2}$, and absence of diabetes mellitus) (Figures 7 and 8). This analysis demonstrates that lower eGFR is associated with increased lifetime risk for ESKD in all demographic subgroups. For eGFR of $90 \mathrm{~mL} / \mathrm{min}$ per $1.73 \mathrm{~m}^{2}$ or greater and other optimal characteristics (see above), lifetime risk for white men and white women was less than $1 \%$ at all ages, but was higher among young black men and women (Figure 8). Lifetime risk for eGFR 60 to $89 \mathrm{~mL} / \mathrm{min}$ per $1.73 \mathrm{~m}^{2}$ was less than $1 \%$ at ages older than 60 years. While these displays are useful for visualizing the impact of baseline eGFR on ESKD risk, we endorse consideration of eGFR as part of the assessment of predicted long-term ESKD risk based on a donor candidate's complete demographic and health profile (as opposed to consideration of single risk factors in isolation).

\section{GFR after Kidney Donation}

GFR declines after kidney donation. A person with a predonation GFR of at least $90 \mathrm{~mL} / \mathrm{min}$ per $1.73 \mathrm{~m}^{2}$ would be expected to have a 1-year postdonation GFR of at least $60 \mathrm{~mL} / \mathrm{min}$ per $1.73 \mathrm{~m}^{2}$. In general, a donor immediately loses approximately $50 \%$ of renal mass, but there is rapid compensatory hyperfiltration leading to a net reduction in GFR of approximately $30 \%(25 \%$ to $40 \%)$ after donation (decrement in GFR of 25 to $40 \mathrm{~mL} / \mathrm{min}$ per $1.73 \mathrm{~m}^{2}$ ). ${ }^{139-141}$

In prior guidelines a GFR level of $80 \mathrm{~mL} / \mathrm{min}$ is frequently cited as the minimal threshold for an acceptable level of kidney function for donation. ${ }^{38,142}$ Limited data show a higher risk for lower GFR after donation among kidney donors with lower predonation GFR. ${ }^{3}$ (Evidence Report Table 16, SDC, http://links.lww.com/TP/B434, and Supplemental Appendix Table D13, SDC, http://links.lww.com/TP/B432).

There is theoretical justification for concern about development of kidney disease after nephrectomy. In experimental animals, hemodynamic alterations associated with
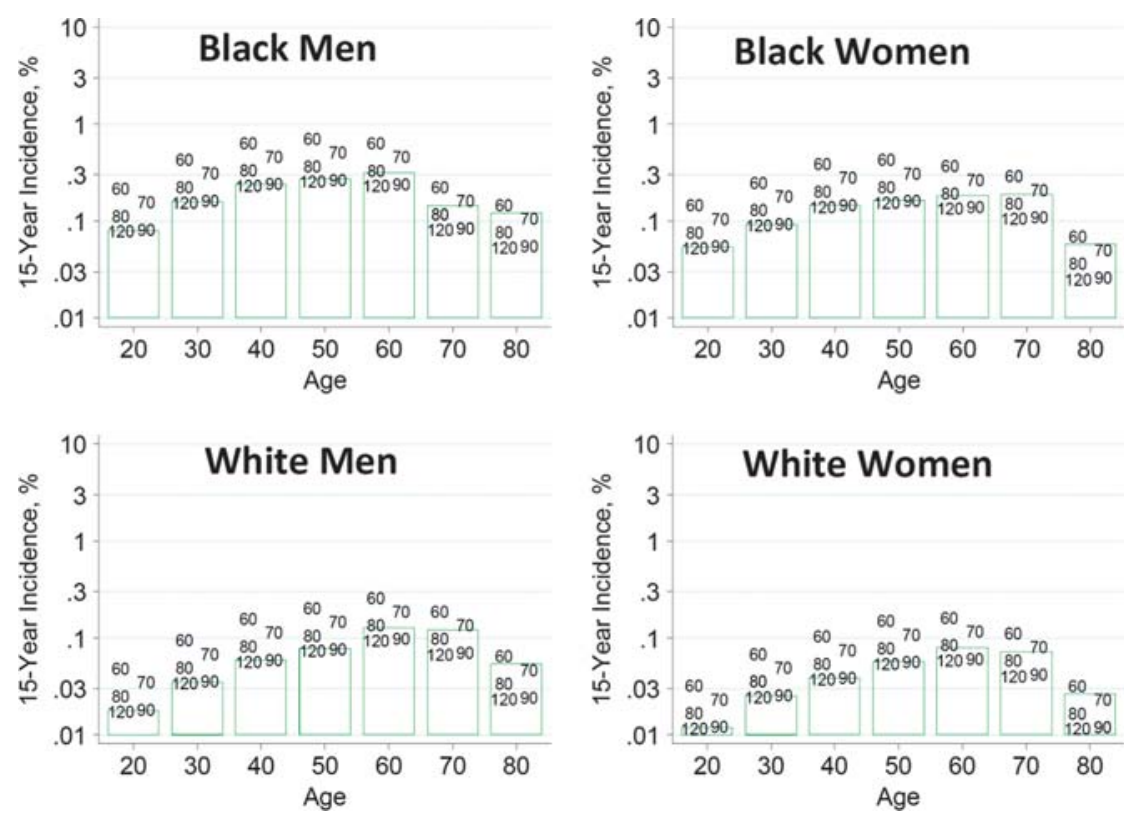

FIGURE 7. Estimated 15-year incidence (\%) of ESKD in the United States according to baseline eGFR and demographic profile from the CKD-PC. *The base-case scenario is defined as: age-specific eGFR $\left(114,106,98,90,82,74\right.$, and $66 \mathrm{~mL} / \mathrm{min}$ per $1.73 \mathrm{~m}^{2}$ for ages $20,30,40,50,60$ 70 , and 80 years, respectively), SBP $120 \mathrm{~mm} \mathrm{Hg}$, urine ACR $4 \mathrm{mg} / \mathrm{g}$ [0.4 mg/mmol], BMl $26 \mathrm{~kg} / \mathrm{m}^{2}$, and no diabetes mellitus or antihypertensive medication use. These were selected as being representative of recent US living kidney donors where, with the exception of eGFR, there was little variation in health characteristics by age. ACR, albumin-to-creatinine ratio; BMI, body mass index; CKD-PC, Chronic Kidney Disease-Prognosis Consortium, eGFR, estimated glomerular filtration rate; ESKD, end-stage kidney disease; SBP, systolic blood pressure. Reprinted from Grams ME, Sang Y, Levey AS, et al. Kidney-failure risk projection for the living kidney-donor candidate. $N$ Engl J Med. 2016;374:411-421. 

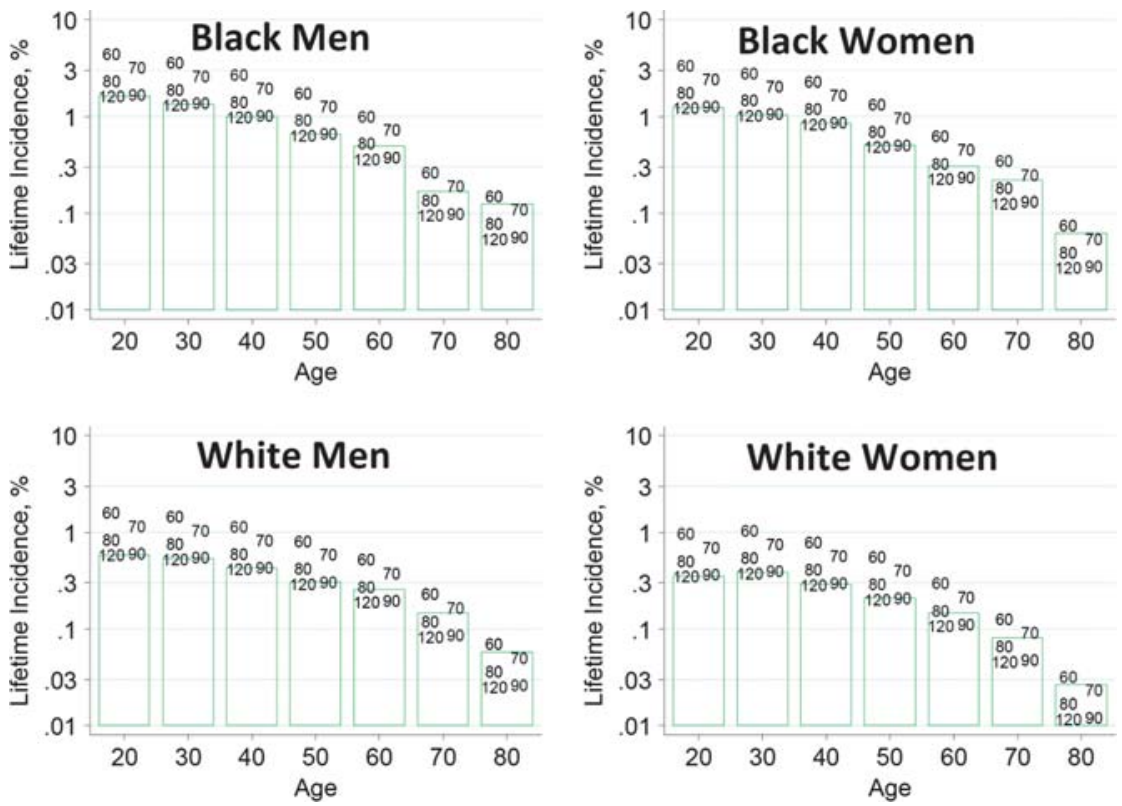

FIGURE 8. Estimated lifetime incidence (\%) of ESKD in the United States according to baseline eGFR and demographic profile from the CKD-PC. *The base-case scenario is defined as: age-specific eGFR $\left(114,106,98,90,82,74\right.$, and $66 \mathrm{~mL} / \mathrm{min}$ per 1.73 $\mathrm{m}^{2}$ for ages 20, 30, 40, 50, 60, 70, and 80 years, respectively), SBP $120 \mathrm{~mm} \mathrm{Hg}$, urine ACR $4 \mathrm{mg} / \mathrm{g}[0.4 \mathrm{mg} / \mathrm{mmol}], \mathrm{BMl} 26 \mathrm{~kg} / \mathrm{m}^{2}$, and no diabetes mellitus or antihypertensive medication use. These were selected as being representative of recent US living kidney donors where, with the exception of eGFR, there was little variation in health characteristics by age. Lifetime risk projections are based on 15 years of follow-up data and calibrated to the incidence of ESKD in the low-risk population, and thus are likely imprecise. ACR, albumin-to-creatinine ratio; BMI, body mass index; CKD-PC, Chronic Kidney Disease-Prognosis Consortium, eGFR, estimated glomerular filtration rate; ESKD, end-stage kidney disease; SBP, systolic blood pressure. Reprinted from Grams ME, Sang Y, Levey AS, et al. Kidney-failure risk projection for the living kidney-donor candidate. N Engl J Med. 2016;374:411-421.

hyperfiltration after reduction in renal mass are followed by development of structural and functional abnormalities associated with kidney disease. In general, the severity of reduction in renal mass is directly associated with the rate of development of subsequent kidney disease. A recent study in humans documents similar hemodynamic alterations associated with hyperfiltration after kidney donation. ${ }^{143,144}$

The risk of ESKD after kidney donation does not exceed ESKD risk in the general population. ${ }^{140,145,146}$ An analysis of living kidney donors in the United States between 1994 and 2003 quantified a postdonation ESKD rate of 0.134 per 1000 person-years over an average follow-up of 9.8 years, which was not higher than the ESKD rate in the general population, even though GFR is lower. ${ }^{147}$ However, the general population is a limited comparison group given that donors undergo careful medical evaluation and selection. ${ }^{4}$ The ERT identified 2 recent studies suggesting that donation is associated with an increase in the risk of ESKD compared to risk in nondonors selected for baseline good health, although the risk increase is small and the absolute postdonation risk remains low (Supplemental Appendix Table D5, SDC, http://links.lww.com/TP/B432). The quality of this evidence was rated as moderate. In comparing 1901 kidney donors with 32621 healthy, demographically matched controls, Mjøen et al reported that $0.47 \%$ of donors $(n=9)$ developed ESKD versus $0.07 \%$ of healthy nondonors $(n=22)$ over a median 15.2-year follow-up. ${ }^{32}$ Based on linking 96217 donors from the US donor registry and healthy participants drawn from the National Health and Nutrition Examination Survey III to national ESKD reporting forms, Muzzale et al estimated that the cumulative incidence of ESKD at 15 years was 30.8 per 10000 in donors compared with 3.9 per 10000 in matched donors (risk attributable to donation of 26.9 per 10 000). ${ }^{30}$ In this study, the incidence of ESKD was higher in individuals who are older versus younger at the time of donation, in men versus women, in blacks versus whites, and in biologically related versus unrelated donors, but risk based on predonation eGFR was not reported. Based on these findings, the likelihood of a small increase in ESKD risk should be discussed with donor candidates; such counseling is endorsed by a 2015 AST Live Donor Community of Practice consensus statement, ${ }^{148}$ and is required in the US by the OPTN Informed Consent Policy beginning in 2017..$^{1}$

\section{Single Kidney GFR}

Many factors determine the preferred kidney to remove for transplantation. If GFR is acceptable, but there are parenchymal, vascular or urological abnormalities or asymmetry in kidney function, it is preferable to transplant the more severely affected kidney or the kidney with lesser function (see also chapter 16).

On average, kidney function and size are correlated. The average length and volume of a single kidney in healthy adults are approximately $11 \mathrm{~cm}$ and $150 \mathrm{~mL}$, respectively, but vary based on age, sex, and body size. ${ }^{149-151}$ On average the normal right kidney is approximately $5 \%$ smaller than the normal left kidney. Asymmetry in kidney size is generally considered as a difference in kidney size greater than $10 \%$ (for example, a difference in kidney length $>1.1 \mathrm{~cm}$ or kidney volume $>15 \mathrm{~mL}$ ). An equivalent difference in kidney function would be greater than $10 \%$ ( $>55 \%$ vs $<45 \%$ in split kidney function). Based on low quality evidence, 1 prior guideline suggested considering a radionuclide imaging study if the difference between kidney lengths is greater 
than $2 \mathrm{~cm}$, and that a difference in function of $10 \%$ or greater between the kidneys may be considered significant. ${ }^{48}$ No studies were found meeting criteria for review by the ERT.

\section{What Prior Guidelines Recommend}

Some guidelines recommend that GFR of $80 \mathrm{~mL} / \mathrm{min}$ or greater is acceptable for donation, based on the level of GFR in the donor (not adjusted for BSA) that was associated with acceptable outcomes in the recipient, not the donor. ${ }^{152}$ Alternatively, some guidelines recommend a GFR level within 2 standard deviations of normal for age and sex. In general, the guidelines do not specify the GFR measurement method to be used, whether the threshold value should be adjusted for BSA, or provide standardized reference values based on sex, race, and age. ${ }^{153}$

A 2007 survey of practices by transplant programs in the United States revealed that approximately $90 \%$ of programs used $\mathrm{mCrCl}$ to measure kidney function, while the other $10 \%$ of programs used the clearance of an exogenous filtration marker, and that approximately $67 \%$ of transplant programs used a threshold of $80 \mathrm{~mL} / \mathrm{min}$ or more to accept donors, while $25 \%$ used a threshold based on age and sex. ${ }^{154}$

In our view, there is not sufficient evidence to justify a single threshold value of $80 \mathrm{~mL} / \mathrm{min}$ not adjusted for BSA, nor age-specific thresholds. In contrast, our recommendations are more consistent with accepted measurement methods and thresholds in general clinical practice, and acknowledge that there is variation in GFR measurement methods and uncertainty in the appropriate threshold to accept or decline donor candidates. For this reason, we recommend GFR measurement by urinary or plasma clearance of specific exogenous filtration markers, which are known to be more accurate than $\mathrm{mCrCl}$, but allow other methods. We recommend a higher threshold value of GFR $(\geq 90 \mathrm{~mL} / \mathrm{min}$ per $1.73 \mathrm{~m}^{2}$ ) to routinely accept a donor candidate, and lower threshold value of GFR $\left(<60 \mathrm{~mL} / \mathrm{min}\right.$ per $\left.1.73 \mathrm{~m}^{2}\right)$ to routinely decline a donor candidate, and a wide intermediate range of GFR $\left(60-89 \mathrm{~mL} / \mathrm{min}\right.$ per $\left.1.73 \mathrm{~m}^{2}\right)$ in which transplant programs can individualize decisions based on other risk factors. Of note, this intermediate range would generally include a $\mathrm{mCrCl}$ of $80 \mathrm{~mL} / \mathrm{min}$ as well as previously recommended age and sex thresholds for mGFR.

\section{RESEARCH RECOMMENDATIONS}

- Evaluate the accuracy of eGFR $\mathrm{cr}_{\mathrm{c}} \mathrm{eGFR}_{\mathrm{cys}}$ and eGFR $\mathrm{Gr}_{\text {-cys }}$ for the prediction of $\mathrm{mGFR}$ in the evaluation and selection of living donor candidates.

- Evaluate long-term risks, including lifetime risk of ESKD, in living donor candidates and living donors according to predonation GFR.

\section{CHAPTER 6: PREDONATION ALBUMINURIA}

The ERT search parameters did not identify evidence from eligible studies pertinent to the recommendations in chapter 6 and therefore the following recommendations are "Not Graded." Some of the recommendations extrapolated from the 2012 KDIGO CKD Guideline ${ }^{124}$ were not part of the ERT review for this guideline and as such they are also "Not Graded."

\section{Evaluation}

6.1: Donor proteinuria should be measured as albuminuria, not total urine protein.

6.2: Initial evaluation of donor albuminuria (screening) should be performed using urine albumin-to-creatinine ratio (ACR) in a random (untimed) urine specimen.

6.3: Donor albuminuria should be confirmed using:

- Albumin excretion rate (AER, $\mathrm{mg} / \mathrm{day}[\mathrm{mg} / \mathrm{d}])$ in a timed urine specimen

- Repeat ACR if AER cannot be obtained.

\section{Selection}

6.4: Urine AER less than $30 \mathrm{mg} / \mathrm{d}$ should be considered an acceptable level for donation.

6.5: The decision to approve donor candidates with AER 30 to $100 \mathrm{mg} / \mathrm{d}$ should be individualized based on demographic and health profile in relation to the transplant program's acceptable risk threshold.

6.6: Donor candidates with urine AER greater than $100 \mathrm{mg} / \mathrm{d}$ should not donate.

\section{RATIONALE}

\section{Evaluation}

The goals of the evaluation of albuminuria in living donor candidates are to:

- Provide an accurate assessment of the amount of albuminuria and long-term risk of ESKD based on albuminuria and other factors.

- Exclude donor candidates whose postdonation risk is expected to exceed the acceptable risk threshold for ESKD established by the transplant program.

- Provide counseling regarding level of risk for donor candidates whose long-term risk for ESKD is expected to be below the acceptable risk threshold established by the transplant program.

- Provide counseling regarding follow-up of albuminuria after donation.

In this section, recommendations are based on physiological principles and recommendations for general clinical practice from the $2012 \mathrm{KDIGO}$ CKD guideline. ${ }^{124}$ There is no evidence to suggest or a reason to think that kidney donors differ from the general population regarding these recommendations.

\section{Proteinuria as a Marker of Kidney Damage}

Urine protein is composed of small amounts of high molecular weight proteins (principally albumin) that are not normally filtered, low molecular weight serum proteins that are normally filtered by the glomeruli and reabsorbed by the tubules, and proteins secreted by the urinary tract.

Increased urinary protein is generally considered a marker of kidney damage: albuminuria reflects increased permeability of the glomeruli (glomerular proteinuria), and low molecular weight proteinuria reflects decreased tubular reabsorption (tubular proteinuria). CKD due to either glomerular or tubulointerstitial diseases is generally associated with both glomerular and tubular proteinuria (albuminuria and low molecular weight proteinuria). Some tubulointerstitial diseases may cause predominantly tubular proteinuria, including Dent disease, toxicity due to heavy metals (eg, cadmium and lead) or aristolochic acid (Balkan and Chinese herb) nephropathy, Sjogren syndrome, multiple myeloma or hereditary diseases 
associated with Fanconi syndrome, and acute tubular necrosis. Conditions other than kidney disease can also cause proteinuria: low molecular weight proteinuria may also reflect overproduction (eg, light chain proteinuria in lymphoproliferative disorders) and high and low molecular weight proteins may arise from increased secretion of urinary tract proteins (due to lower urinary tract diseases).

Urine albumin is the preferred measure of urine protein for assessment of kidney damage. Tests for total urine protein cannot be standardized because they are not traceable to a standard reference material due to the varying composition of urine protein. Current efforts to standardize albuminuria assessment are directed to establishing traceability of tests for urine albumin to standardized reference material for serum albumin. Other urine proteins are less well standardized than albumin.

The albumin loss rate (hereafter referred to as albumin excretion rate, AER) is not regulated in health and is widely accepted as a marker of kidney damage. Increased AER is associated with a wide range of complications and increased AER is one of the criteria for the definition of CKD. In diabetic kidney disease and other glomerular diseases, increased AER generally occurs before the decline in GFR.

\section{Measurement Methods}

The KDIGO 2012 CKD guideline for the evaluation of albuminuria in the general population recommends 2-stage testing (initial testing followed by confirmatory testing). ${ }^{124}$ The current living donor guideline WG concluded that these general recommendations were applicable to kidney donor candidates.

Initial tests, in order of preference, and the rationale are described below. In all cases an early morning urine sample is preferred as it minimizes variation due to diurnal variation in albumin excretion and urine concentration.

\section{Urine Albumin-to-Creatinine Ratio (ACR)}

The rationale for preferring ACR to albumin concentration is that urine concentration and dilution can vary by more than
10 -fold among individuals and during the day. The KDIGO CKD guideline therefore recommends that clinical laboratories measure creatinine when albumin is requested, and express the results as ACR in addition to albumin concentration. Indexing urine albumin by urine creatinine concentration overcomes variation due to urine concentration and dilution, but introduces variation by creatinine generation.

Recently, some investigators have proposed estimating creatinine excretion rate and multiplying this quantity by ACR to estimate AER. ${ }^{155}$ The lower limit of detection for urine albumin in the clinical laboratory where the test is performed can be used for computation of urine ACR if the clinical laboratory reports "below the detectable limit." Factors affecting urine ACR in addition to kidney disease are shown in Table 14.

One study reported excellent performance of ACR to detect AER of $30 \mathrm{mg} / \mathrm{d}$. The area under the receiver operator curve was $0.93 .{ }^{156}$ An ACR threshold of $\geq 10 \mathrm{mg} / \mathrm{g}$ was associated with sensitivity and specificity of $88 \%$ each for detecting AER $\geq 30 \mathrm{mg} / \mathrm{d}$. There was minor variation in area under the receiver operator curve based on age, sex, race, and body weight.

\section{Urine Protein-to-Creatinine Ratio (PCR)}

Tests for total urine protein cannot substitute for tests for urine albumin. PCR is less sensitive than ACR, so even negative tests must be confirmed by tests for albumin. Increased PCR suggests increased ACR, but nonalbumin protein can cause a positive test, so positive tests should be confirmed by tests for albumin. Patients with elevated PCR and negative tests for albumin may have tubular proteinuria, light chain proteinuria or urinary tract disease. Specific assays are available for $\alpha 1$-microglobulin, $\beta 2$ microglobulin, monoclonal heavy or light chains.

\section{Reagent Strip Urinalysis for Total Protein with Automated Reading}

Reagent strips allow point-of-care, semi-quantitative assessment of total urine protein concentration. Reagent strips

\section{TABLE 14.}

\section{Factors affecting urinary ACR}

Factor

Examples of effect

Preanalytical factors

- Transient elevation in albuminuria

- Intraindividual variability

- Preanalytical storage conditions

- Nonrenal causes of variability in creatinine excretion

- Changes in creatinine excretion Analytical factors

- Antigen excess ("prozone") effect

\author{
Menstrual blood contamination \\ Symptomatic UTI \\ Exercise \\ Upright posture (orthostatic proteinuria) \\ Other conditions increasing vascular permeability (eg, septicemia) \\ Intrinsic biological variability \\ Genetic variability \\ Degradation of albumin before analysis \\ Age (lower in children and older people) \\ Race (lower in white than black people) \\ Muscle mass (eg, lower in people with amputations, paraplegia, muscular dystrophy) \\ Gender (lower in women) \\ Non-steady state for creatinine (AKI)
}

Samples with very high albumin concentrations may be falsely reported as low or normal using some assays 
(“dipsticks") are more sensitive to albumin than other proteins, but lack specificity. Automated readers are more accurate than manual reading of reagent strips.

\section{Reagent Strip Urinalysis for Total Protein with Manual Reading, if the Above Measures are not Available}

Because of variability in urine ACR and its relationship to urine AER, the WG recommends confirmation in all cases. The preferred confirmatory test is urine AER, expressed as $\mathrm{mg} / \mathrm{d}$. If urine AER is not available, a repeat ACR is acceptable. Consistent with the 2012 KDIGO CKD guideline, testing for specific urine proteins such as $\alpha 1$-microglobulin, $\beta 2$ microglobulin, monoclonal heavy or light chains (also known as "Bence Jones" proteins) can be undertaken if significant nonalbumin proteinuria is suspected. These assays can be performed at the same time as tests for albuminuria.

\section{Selection: Criteria for Acceptable Predonation Albuminuria}

\section{AER in the General Population}

The normal level of AER in healthy young men and women is less than $10 \mathrm{mg} / \mathrm{d}$. The coefficient of variation for repeated measurements is approximately $30 \% .{ }^{157}$ Because of the high coefficient of variation, repeated measurements are preferred for assessment of albuminuria.

AER rises with age, although the cause of rise is not known and the rate of rise appears widely variable. Most data are based on cross-sectional studies and mean AER is higher in older populations than in young populations. As discussed in chapter 5, there are often abnormalities in kidney function and structure in the elderly and there is debate about whether higher AER in older people represents normal aging or disease.

Higher albuminuria in the general population is associated with a higher risk of complications of CKD, including ESKD, CVD and death. In general populations, compared with a reference ACR of $5 \mathrm{mg} / \mathrm{g}(0.5 \mathrm{mg} / \mathrm{mmol})$, the RR for complications related to increased ACR rises at higher ACR, without an apparent threshold when expressed on the log scale. ${ }^{134}$ The risk of higher ACR is independent of the eGFR. Similar relationships of high albuminuria with adverse outcomes have been observed in subgroups including patients with known CKD (Figure 6). ${ }^{134-137}$ KDIGO 2012 CKD guideline defines AER $>30 \mathrm{mg} / \mathrm{d}$ for 3 months or more as satisfying the criteria for CKD. AER less than $30 \mathrm{mg} / \mathrm{d}$ in young men and women is considered normal to mildly increased, with 10 to $29 \mathrm{mg} / \mathrm{d}$ considered as "high normal"; AER 30 to $300 \mathrm{mg} / \mathrm{d}$ is defined as moderately increased compared with the young adult level; and AER greater than $300 \mathrm{mg} / \mathrm{d}$ is defined as severely increased compared with the young adult level. Approximate ranges for other measures of urine protein are as shown in Table 15. The association of higher albuminuria with higher risk of adverse outcomes may be related to other conditions that co-occur with high albuminuria, such as hypertension, diabetes and CVD. The RR for these adverse CKD outcomes (ESKD, CVD, death) in older people with higher urine ACR compared with the reference urine ACR is less than the RR in younger people; however the increment in absolute risk in older people is higher in older people than in younger people. ${ }^{138}$

A recent meta-analysis based on data from nearly 5 million healthy persons identified from 7 general population cohorts found that each 10-fold increase in urinary ACR was associated with 3 times the risk of ESKD over median cohort follow-up of 4 to 16 years, although the finding was not statistical significant (95\% Lower Confidence Limit adjusted hazard ratio $[\mathrm{aHR}] 95 \%$ Upper Confidence Limit; $\left.0.992 .944_{8.75}\right){ }^{7} \mathrm{Af}-$ ter calibration to annual ESKD incidence in the US healthy population, variations in the projected 15-year and lifetime risks of ESKD based on urine ACR were generated according to age, sex, and race for healthy persons (assuming agespecific eGFR, SBP $120 \mathrm{~mm} \mathrm{Hg}$, BMI $26 \mathrm{~kg} / \mathrm{m}^{2}$, and absence of diabetes mellitus; Figures 9 and 10). This analysis demonstrates that higher albuminuria is associated with higher lifetime risk of ESKD in all subgroups, with higher risk in men than women and blacks than whites. For urine ACR less than $10 \mathrm{mg} / \mathrm{g}$ and other optimal risk factors (see above), lifetime risk for white men and white women was less than $1 \%$ at all ages, but exceeded $2 \%$ for black men younger than 40 (Figure 10). For urine ACR less than $30 \mathrm{mg} / \mathrm{g}$, the lifetime

\section{TABLE 15.}

\section{Relationship among categories for albuminuria and proteinuria}

\begin{tabular}{lccc}
\hline & & Categories & \\
\cline { 2 - 4 } Measure & Normal to mildly increased (A1) & Moderately increased (A2) & Severely increased (A3) \\
\hline AER $(\mathrm{mg} / 24 \mathrm{~h})$ & $<30$ & $30-300$ & $>300$ \\
PER $(\mathrm{mg} / 24 \mathrm{~h})$ & $<150$ & $150-500$ & $>500$ \\
$\begin{array}{l}\text { ACR } \\
(\mathrm{mg} / \mathrm{mmol})\end{array}$ & $<3$ & & $>30$ \\
$\quad(\mathrm{mg} / \mathrm{g})$ & $<30$ & $30-300$ & $>300$ \\
PCR & & & $>50$ \\
$\quad(\mathrm{mg} / \mathrm{mmol})$ & $<15$ & $15-50$ & $>500$ \\
$\quad(\mathrm{mg} / \mathrm{g})$ & $<150$ & $150-500$ & + or greater \\
\hline
\end{tabular}

Albuminuria and proteinuria can be measured using excretion rates in timed urine collections, ratio of concentrations to creatinine concentration in spot urine samples, and reagent strips in spot urine samples. Relationships among measurement methods within a category are not exact. For example, the relationships between AER and ACR and between PER and PCR are based on the assumption that average creatinine excretion rate is approximately $1.0 \mathrm{~g} / \mathrm{d}$ or $10 \mathrm{mmol} / \mathrm{d}$. The conversions are rounded for pragmatic reasons. (For an exact conversion from mg/g of creatinine to mg/mmol of creatinine, multiply by 0.113 .) Creatinine excretion varies with age, sex, race, and diet; therefore the relationship among these categories is approximate only. ACR less than $10 \mathrm{mg} / \mathrm{g}(<1 \mathrm{mg} / \mathrm{mmol})$ is considered normal; ACR 10 to $30 \mathrm{mg} / \mathrm{g}$ (1-3 mg/mmol) is considered "high normal." ACR greater than $2200 \mathrm{mg} / \mathrm{g}$ (>220 mg/mmol) is considered "nephrotic range." The relationship between urine reagent strip results and other measures depends on urine concentration. ACR, albumin-to-creatinine ratio; AER albumin excretion rate; PCR, protein-to-creatinine ratio; PER, protein excretion rate. Adapted with permission from Kidney Disease: Improving Global Outcomes (KDIGO) CKD Work Group. KDIGO 2012 clinical practice guideline for the evaluation and management of chronic kidney disease. Kidney Int 2013:1-150. ${ }^{124}$ 

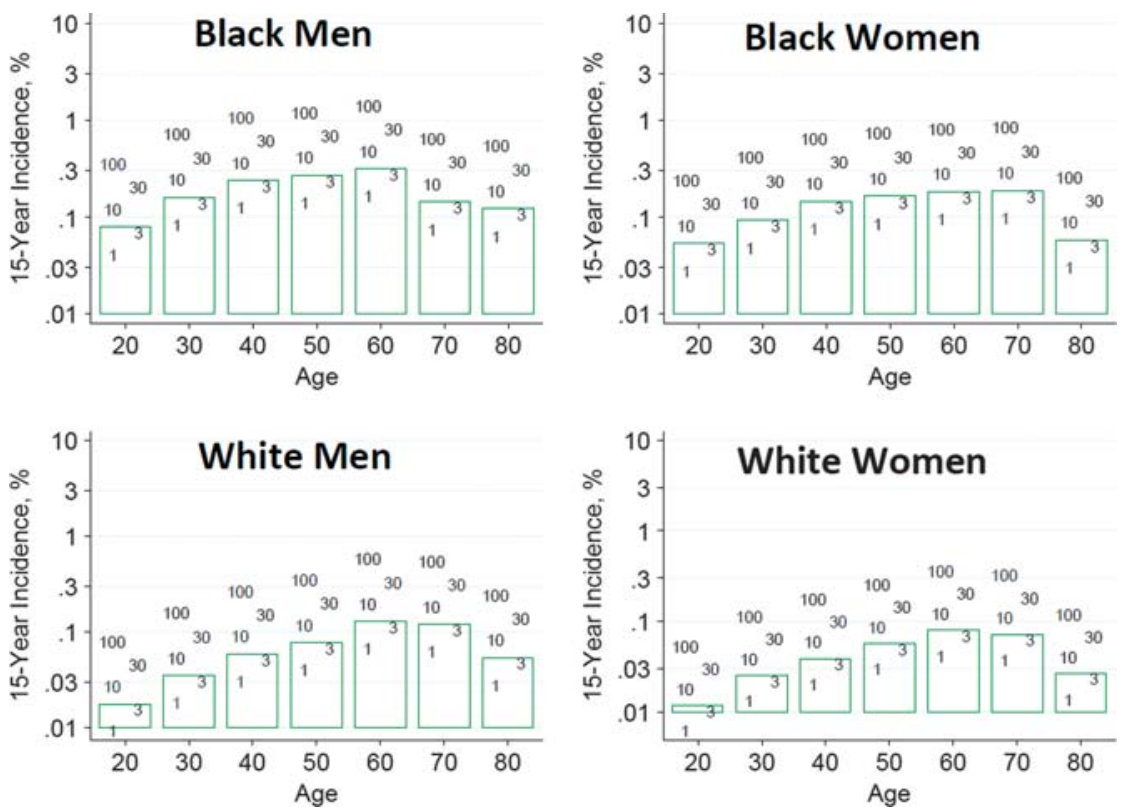

FIGURE 9. Estimated 15-year incidence (\%) of ESKD in the United States according to baseline albumin-to-creatinine ratio (ACR, mg/g) and demographic profile from the CKD-PC. *The base-case scenario is defined as: age-specific eGFR $(114,106,98,90,82,74$, and $66 \mathrm{~mL} / \mathrm{min}$ per $1.73 \mathrm{~m}^{2}$ for ages 20, 30, 40, 50, 60, 70, and 80 years, respectively), SBP $120 \mathrm{~mm} \mathrm{Hg}$, urine ACR $4 \mathrm{mg} / \mathrm{g}[0.4 \mathrm{mg} / \mathrm{mmol}]$, BMl 26 kg/m², and no diabetes mellitus or antihypertensive medication use. These were selected as being representative of recent US living kidney donors where, with the exception of eGFR, there was little variation in health characteristics by age. BMl, body mass index; CKD-PC, Chronic Kidney Disease-Prognosis Consortium, eGFR, estimated glomerular filtration rate; ESKD, end-stage kidney disease; SBP, systolic blood pressure. Reprinted from Grams ME, Sang Y, Levey AS, et al. Kidney-failure risk projection for the living kidney-donor candidate. N Engl J Med. 2016;374:411-421.7

risk was less than $1 \%$ in white men at age $>50$ years, black men at age $>70$, and black women at age $>60$. While these displays are useful for visualizing the associations of baseline ACR with ESKD risk, we endorse consideration of ACR as part of the assessment of predicted long-term ESKD risk based on a donor candidate's complete demographic and health profile (as opposed to consideration of single risk factors in isolation).
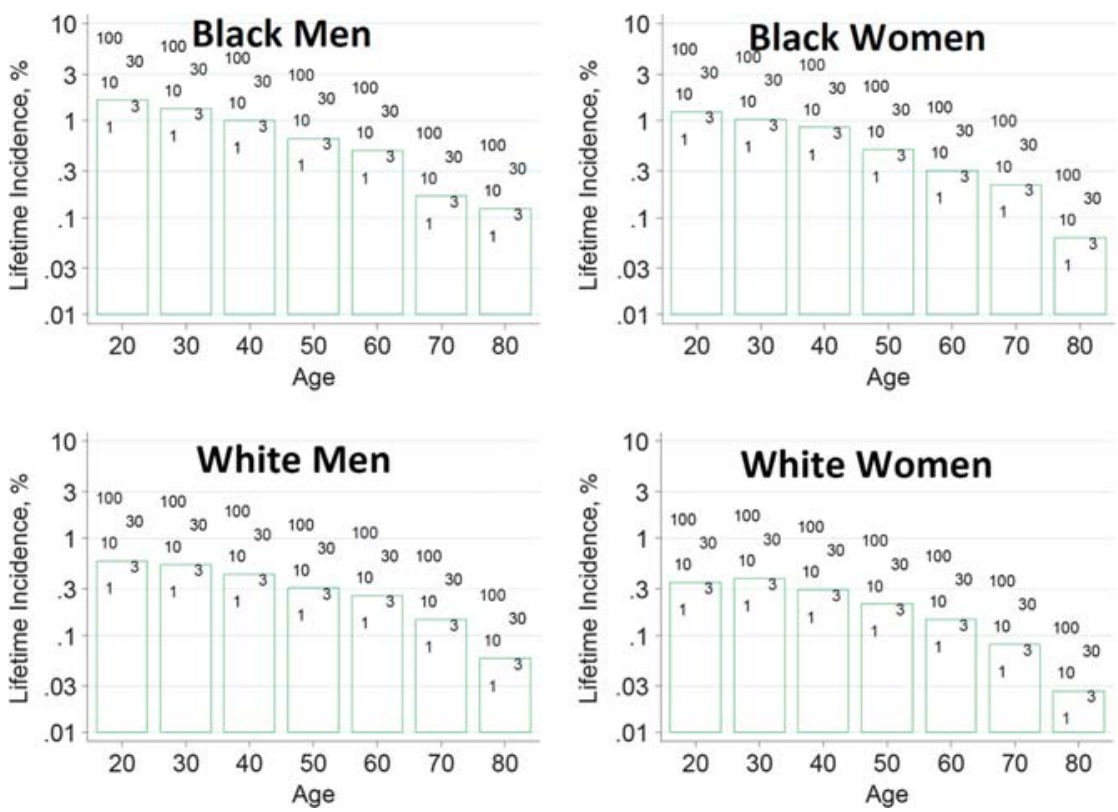

FIGURE 10. Estimated lifetime incidence (\%) of ESKD in the United States according to baseline albumin-to-creatinine ratio (ACR, $\mathrm{mg} / \mathrm{g}$ ) and demographic profile from the CKD-PC. * The base-case scenario is defined as: age-specific eGFR $(114,106,98,90,82,74$, and 66 mL/min per $1.73 \mathrm{~m}^{2}$ for ages 20, 30, 40, 50, 60, 70, and 80 years, respectively), SBP $120 \mathrm{~mm} \mathrm{Hg}$, urine ACR $4 \mathrm{mg} / \mathrm{g}\left[0.4 \mathrm{mg} / \mathrm{mmol}^{\prime}, \mathrm{BMl} 26 \mathrm{~kg} / \mathrm{m}^{2}\right.$, and no diabetes mellitus or antihypertensive medication use. These were selected as being representative of recent US living kidney donors where, with the exception of eGFR, there was little variation in health characteristics by age. Lifetime risk projections are based on 15 years of follow-up data and calibrated to the incidence of ESKD in the low-risk population, and thus are likely imprecise. BMI, body mass index; CKDPC, Chronic Kidney Disease-Prognosis Consortium, eGFR, estimated glomerular filtration rate; ESKD, end-stage kidney disease; SBP, systolic blood pressure. Reprinted from Grams ME, Sang Y, Levey AS, et al. Kidney-failure risk projection for the living kidney-donor candidate. N Engl J Med. 2016;374:411-421. ${ }^{7}$ 


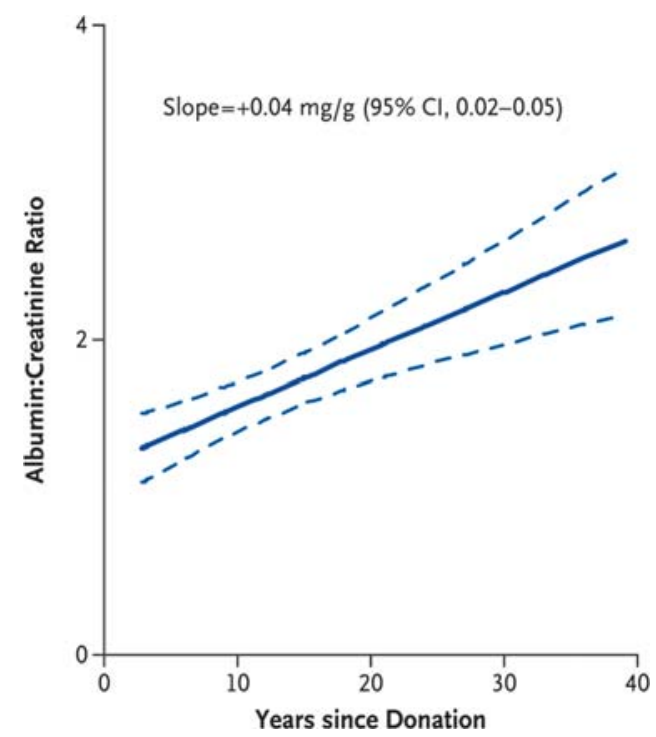

FIGURE 11. Proteinuria after kidney donation. Solid line, regression line; dotted line: 95\% confidence interval. Reprinted with permission of the Massachusetts Medical Society from Ibrahim HN, Foley R, Tan L, et al. Long-term consequences of kidney donation. N Engl $J$ Med. 2009;360:459-469. ${ }^{140}$

\section{Proteinuria after Kidney Donation}

Some but not all studies demonstrate donors have an increase in proteinuria compared with nondonor control groups (Figures 11 to 12$){ }^{139-141}$ In prior guidelines a protein excretion rate $(\mathrm{PER})<150 \mathrm{mg} / \mathrm{d}$ was frequently cited as acceptable for donation. ${ }^{48,50,158,159}$ This level corresponds roughly to AER less than $30 \mathrm{mg} / \mathrm{d}$, which includes normal and mildly increased (Table 15). ${ }^{124}$ However, the risk associated with albuminuria in kidney donors is uncertain.

There is theoretical justification for concern about development of kidney disease after nephrectomy. First, in experimental animals, reduction in renal mass is associated with increased glomerular permeability to albumin followed by other structural and functional abnormalities associated with kidney disease. Second, given that GFR declines after kidney donation, the filtered load of albumin would be expected to decline. Unchanged or higher albuminuria after donation suggests increased albumin filtration per nephron.

In a prior systematic review, the incidence of clinical proteinuria after donation was quantified in 42 studies, that followed 4793 living donors for an average of 7 years (range, 2-25 years). ${ }^{141}$ There was significant heterogeneity between the studies $(P<0.0001)$. Some studies reported an incidence of proteinuria over $20 \%$, whereas in others the incidence was less than $5 \%$. The pooled incidence of proteinuria was $12 \%(95 \% \mathrm{CI}$, $8-16 \%)$. These results were similar in a supplementary analysis limited to 9 studies which consistently defined proteinuria as greater than $300 \mathrm{mg} / \mathrm{d}$ based on 24-hour urine. The pooled incidence of proteinuria among these 9 studies, which followed a total of 1799 donors for 7 years, was 10\% (95\% CI, 7-12\%).

\section{What Prior Guidelines Recommend}

Some guidelines recommend that accepted living donor candidates have a PER less than 150 to $300 \mathrm{mg}$ per day, based on the usually accepted normal range, generally without reference to measurement methods. A survey of practices by transplant programs in the United States reported in 2007 found that approximately $76 \%$ of programs used a PER in a 24-hour urine collection for donor evaluation, and that $36 \%$ used PER > $150 \mathrm{mg} / \mathrm{d}$ as a threshold for donor exclusion (unless proteinuria is postural), while $44 \%$ reported higher exclusion thresholds of 300-1000 mg/d. ${ }^{154}$

By comparison, our recommendations are more consistent with the recently accepted criterion standard, measurement methods and thresholds in general clinical practice, but acknowledge that there is variation in ascertainment of albuminuria for screening and uncertainty in the appropriate threshold to accept or decline donor candidates. For these reasons, we recommend measurement of albumin rather than total protein, and AER in a timed urine collection rather than ACR in a spot urine specimen if possible. We recommend an AER threshold of less than $30 \mathrm{mg} / \mathrm{d}$ to routinely accept a donor candidate, which corresponds to normal to mildly increased. We recommend an intermediate range of AER 30 to $100 \mathrm{mg} / \mathrm{d}$ corresponding to the lower range for moderately increased AER, in which to individualize decisions based on other risk factors. We acknowledge that AER of 30 to $100 \mathrm{mg} / \mathrm{d}$ meets the criteria for CKD, and past recommendations have strived to exclude donor candidates with CKD. We have not excluded candidates solely on the basis of AER 30 to $100 \mathrm{mg} / \mathrm{d}$ because estimated projected predonation lifetime risk of ESKD in older persons with ACR in this range is very low in the absence of decreased GFR and other health risk factors. ${ }^{7}$ Thus, we concluded that universal exclusion would not be consistent with a Framework that would allow donation from other candidates with similar risk due to other clinical risk factors.

We do not require tests of total urine protein in addition to albumin because there are no accepted normal ranges for urine nonalbumin protein excretion; disorders associated with predominant tubular proteinuria (tubulointerstitial kidney disease) and overproduction proteinuria (plasma cell or B-lymphocyte disorders) are uncommon, and patients with these disorders usually have other clinical abnormalities that would be discovered during the donor evaluation.

\section{RESEARCH RECOMMENDATIONS}

- Assess the accuracy of urine ACR compared with AER for evaluation and selection of living donor candidates.

- Evaluate the association of urine albumin with other kidney measures (eg, GFR and kidney size) at the time of donor evaluation and with kidney measures and outcomes (eg, GFR change, ESKD, survival) after donation.

\section{CHAPTER 7: PREDONATION HEMATURIA}

The ERT search parameters did not identify evidence from eligible studies pertinent to the recommendations in chapter 7 and therefore the following recommendations are "Not Graded.”

\section{Evaluation}

7.1: Donor candidates should be assessed for microscopic hematuria.

7.2: Donor candidates with persistent microscopic hematuria should undergo testing to identify possible causes, which may include:

- Urinalysis and urine culture to assess for infection 


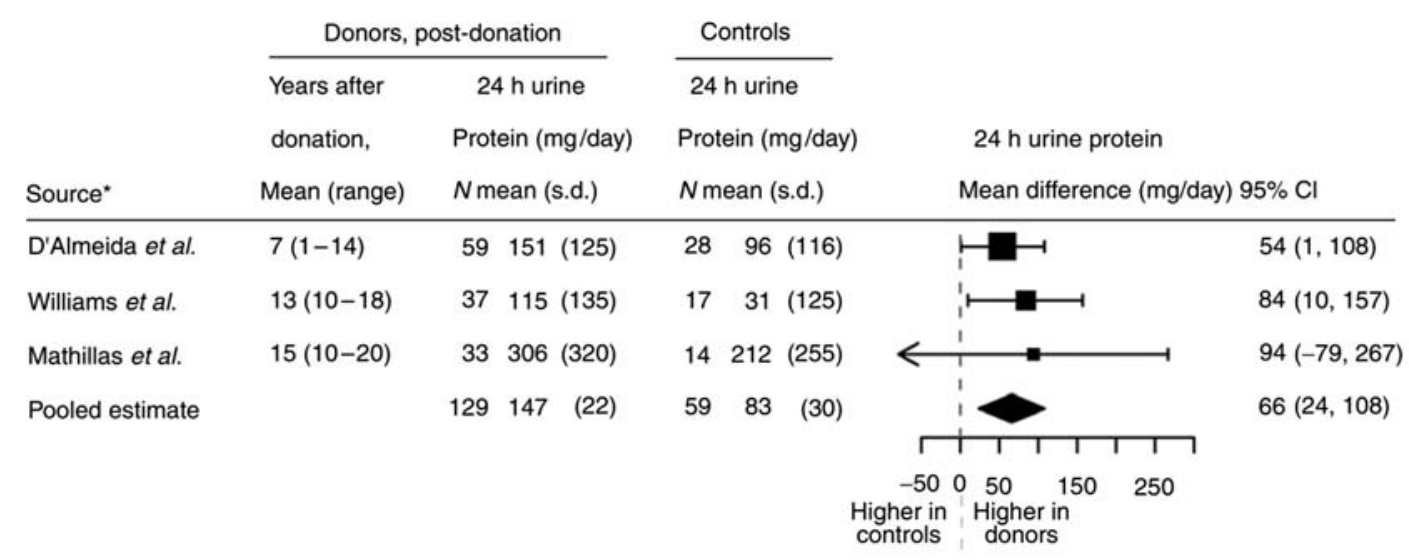

$24 \mathrm{~h}$ urine albumin $\dagger$

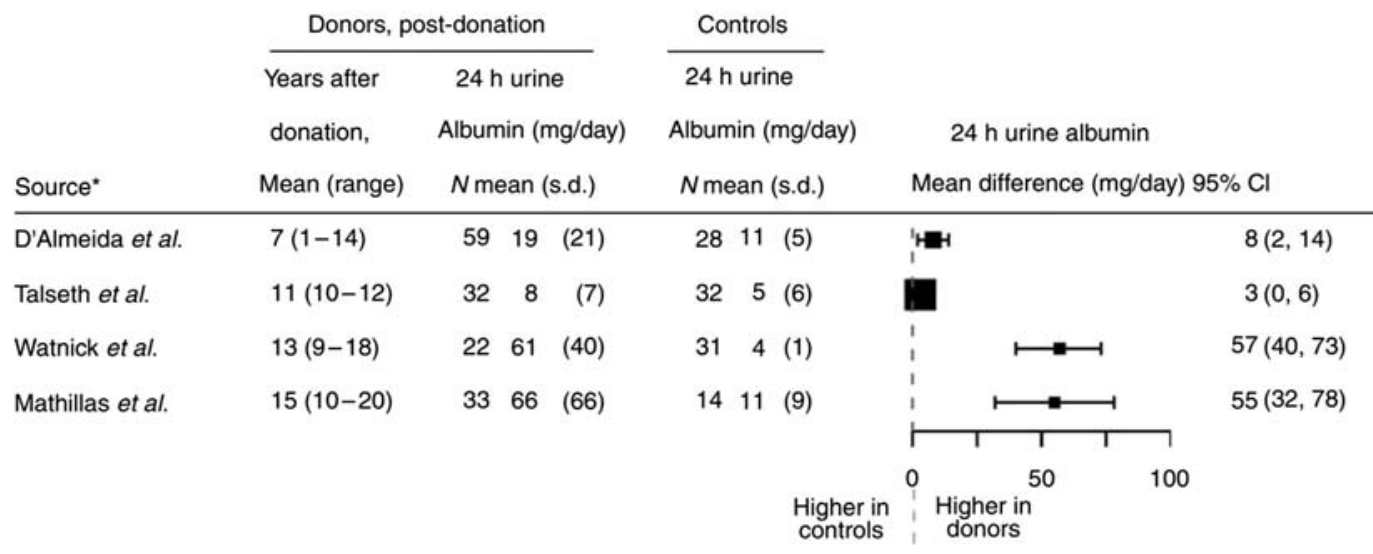

Microalbuminuria \$

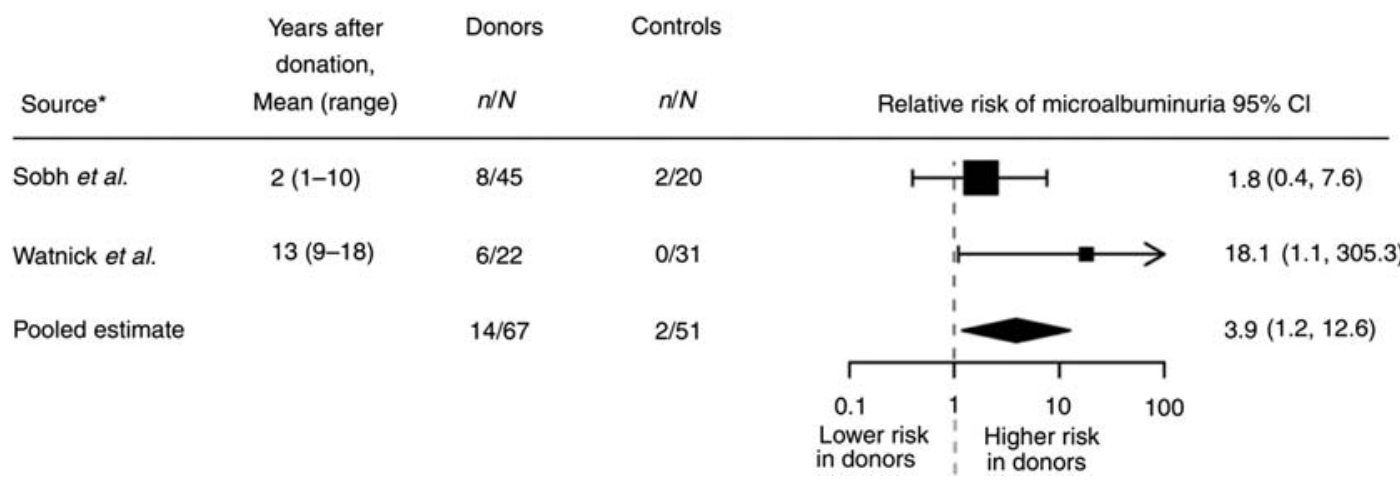

FIGURE 12. Meta-analysis of proteinuria after kidney donation. Controlled studies of proteinuria after kidney donation. The size of each square is inversely proportional to the variability of the study estimate. *Studies are arranged by the average number of years after donation. $\ddagger$ Microalbuminuria was assessed by 24 -hour urine. †Mathematically pooled results are not presented graphically because of statistical heterogeneity between studies. Cl, confidence interval. Reprinted with permission from Garg AX, Muirhead N, Knoll G, et al. Proteinuria and reduced kidney function in living kidney donors: a systematic review, meta-analysis, and meta-regression. Kidney Int. 2006;70:1801-1810. ${ }^{14}$

- Cystoscopy and imaging to assess for urinary tract malignancy

- 24-hour urine stone panel to assess for nephrolithiasis and/or microlithiasis

- Kidney biopsy to assess for glomerular disease (eg, thin basement membrane nephropathy, IgA nephropathy, Alport syndrome)

\section{Selection}

7.3: Donor candidates with hematuria from a reversible cause that resolves (eg, a treated infection) may be acceptable for donation.
7.4: Donor candidates with IgA nephropathy should not donate.

\section{RATIONALE}

\section{Evaluation and Definitions}

Persistent microscopic hematuria is most often defined as more than 2 to 5 red blood cells per high-power field of urinary sediment on 2 to 3 separate occasions, unrelated to exercise, trauma, sexual activity or menstruation. ${ }^{160-163}$ Consensus-based guidelines of the American Urological 
Association state that while a positive dipstick reading warrants microscopic examination to confirm the diagnosis of asymptomatic microhematuria, a positive dipstick alone does not define microhematuria, and evaluation should be based solely on findings from microscopic examination of urinary sediment. ${ }^{161}$ Causes of a positive dipstick reading in the absence of red blood cells in the urine include hemoglobinuria, myoglobinuria, a dilute urine sample, or simply a false-positive test.

The estimated prevalence of microscopic hematuria varies widely from $0.18 \%$ to $16 \% .{ }^{160}$ A recent population study of 1.2 million persons aged 16 to 25 years in Israel identified prevalent asymptomatic persistent microscopic hematuria in $0.3 \%$ of individuals. ${ }^{163}$ Persistent microscopic hematuria may be associated with urologic abnormalities (eg, stones, tumors) or glomerular disease. The most common glomerular causes of persistent isolated microscopic hematuria are $\operatorname{IgA}$ nephropathy, thin basement membrane nephropathy (TBMN) and Alport syndrome. ${ }^{164-167}$

There are consensus-based guidelines for the evaluation of asymptomatic microhematuria in the general population. For example, the 2012 recommendations from the American Urological Association ${ }^{161}$ include assessment of risk factors for urinary tract malignancies (eg, irritative voiding symptoms, current or past tobacco use, chemical exposures), radiological evaluation (eg, multiphasic computed tomography urography, without and with intravenous contrast, or magnetic resonance urography), and cystoscopy in patients age 35 years or older regardless of history of use of anticoagulation therapy. Urine cytology and urine biomarkers are not recommended as a part of the routine evaluation of asymptomatic microhematuria.

The presence of dysmorphic urinary red blood cells detected by conventional microscopy, phase-contrast microscopy, or automated analyzer has a broad a range of sensitivities (32\% to $100 \%$ ) and specificities (from $33 \%$ to $100 \%$ ) for glomerular causes of microhematuria. ${ }^{161}$ The presence of dysmorphic red blood cells or cellular urinary casts and other clinical information can be used to prioritize the evaluation for glomerular causes of hematuria. However, the presence of dysmorphic red blood cells does not exclude underlying urologic disease. ${ }^{161}$

Although isolated microscopic hematuria in young persons is often considered "benign," a recent populationbased study of 1.2 million Israeli persons aged 16 to 25 years with up to 35 years of follow-up identified small but significant increase in long-term renal risk associated with persistent asymptomatic isolated microscopic hematuria, with ESKD rates of 34.0 versus 2.05 per 100000 person-years among those with versus without persistent microscopic hematuria (adjusted HR, 12.418.527.6). ${ }^{163}$ While participants were required to have serum creatinine values "within the normal range" and 24-hour urine protein less than $200 \mathrm{mg}$, this study does not provide information on ESKD risk after comprehensive evaluation and selection including measured kidney function.

\section{Selection}

The sequence of the evaluation for microscopic hematuria, like other testing in this guideline, is designed to perform less invasive/expensive tests before more invasive/expensive tests, and only if the less invasive/expensive tests do not preclude donation (Figure 13). ${ }^{168}$ Reversible causes of microscopic hematuria, for example, treatable urinary tract infection, should generally not preclude donation. In the absence of a family history suggesting possible TBMN or Aport disease, additional testing to rule out these causes is generally not

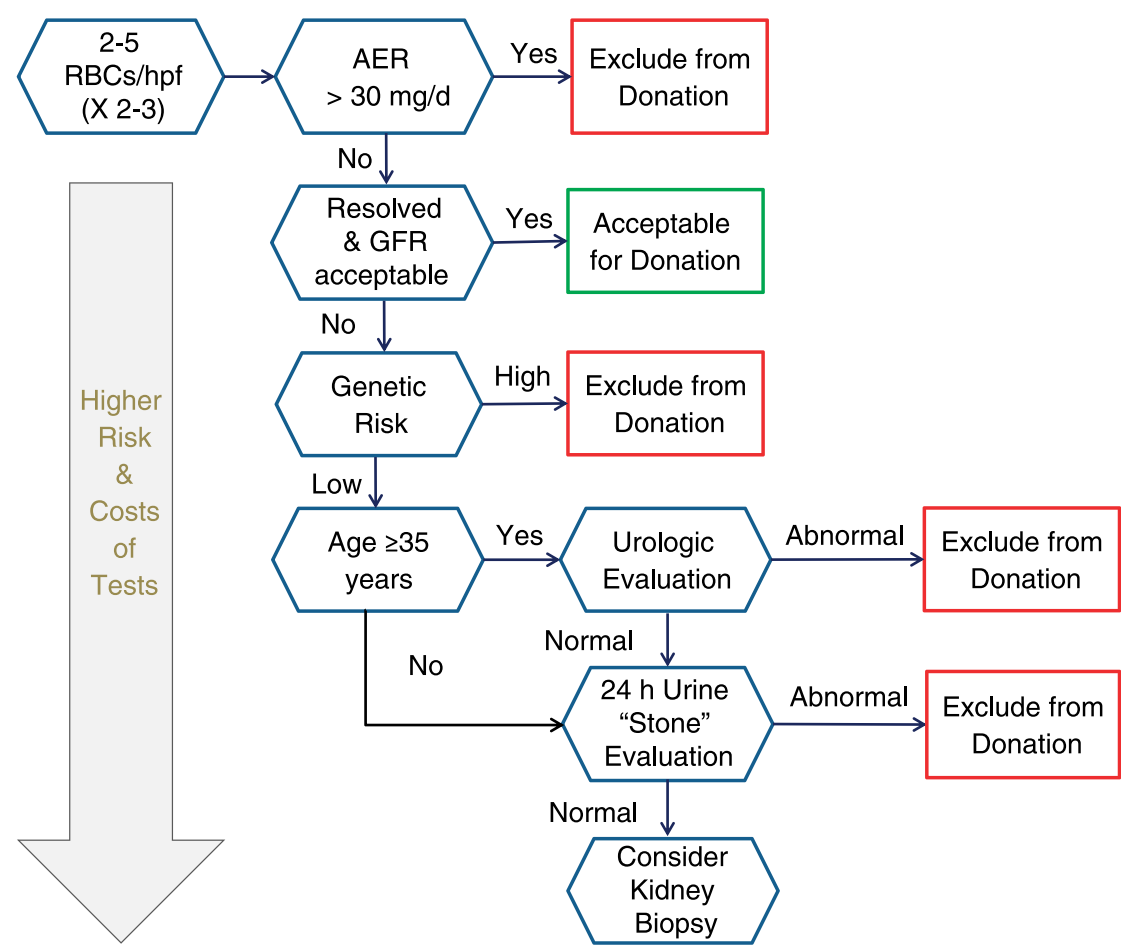

FIGURE 13. Sequential evaluation of microscopic hematuria in living kidney donor candidates. In general, lower risk and less expensive tests should be performed first, and at each step additional testing should only be performed if necessary. Boxes indicate stopping points in the donor candidate hematuria evaluation. AER, albumin excretion rate; GFR, glomerular filtration rate; hpf, high-power field; RBC, red blood cell. 
necessary. Please refer to chapter 14 for a related discussion on genetic testing in the donor candidate.

Epidemiological studies of TBMN suggest increased risks of hypertension and proteinuria compared with the general population over time, but progression to ESKD is rare and thought to require an additional insult. ${ }^{169}$ Data on outcomes of living kidney donor evaluation and donation in persons with TBMN are limited to small series with short-term follow-up. ${ }^{170,171}$ A series of 512 consecutive donors at a US center identified asymptomatic, microscopic hematuria for at least 1 month in $2.7 \%(n=14)$. Hematuria resolved after treatment for urinary tract infection in 2. Kidney biopsy was performed in 10/12, and showed: TBMN (5/12); normal (2/12); nonhomogeneous basement membrane abnormalities (1/12); IgA nephropathy (1/12); and greater than $20 \%$ glomerulosclerosis in a patient with a family history of Schimke's syndrome (immune-osseous dysplasia). Two of the 4 with TBMN, aged 44 and 53 years, proceeded with donation; after 15 months follow-up, donors were free of hypertension, proteinuria, and recipients had "excellent" graft function. ${ }^{170}$ A Korean series including 5 living donors with TBMN defined by predonation biopsy reported favorable short-term outcomes, including mean serum creatinine $0.94 \pm 0.32 \mathrm{mg} / \mathrm{dL}(83 \pm 28.3 \mu \mathrm{mol} / \mathrm{L})$ and no cases of new-onset hypertension or proteinuria over mean follow-up period of $34.7 \pm 42.5$ months. ${ }^{171}$

Notably, TMBN is often defined based on pathological description rather than as a distinct clinical entity. Carrier states for Alport mutations may present as TBMN. High rates of proteinuria $(75 \%)$ and ESKD $(8-30 \%)$ have been reported in female carriers of X-linked Alport syndrome mutations. ${ }^{172}$ A recent study identified adverse kidney outcomes in 234 Alport carriers (including 29 autosomal recessive and 205 X-linked mutation carriers): ESKD developed in $17.5 \%$ at a median age of 49 years, and outcomes including $\mathrm{ESKD}$, proteinuria and impaired kidney function were similar in X-linked and autosomal recessive carriers, ${ }^{173}$ although the number of autosomal recessive carriers was small. Among 6 female Alport carriers ( 5 X-linked, 1 autosomal recessive) who donated to their children at several European centers and were followed for an average 6.7 years, 3 developed new-onset hypertension and 2 developed new-onset of proteinuria. ${ }^{174}$ Creatinine clearance remained greater than $40 \mathrm{~mL} / \mathrm{min}$ in all donors after up to 14 years. Thus, while data are limited, female carriers of X-linked Alport syndrome (ie, COL4A5 mutation) should be discouraged from kidney donation because of their own increased risk of hypertension and adverse kidney outcomes even in the absence of donation.

IgA nephropathy that presents with hematuria and minimal proteinuria is often a progressive disease. ${ }^{175}$ In one series in Hong Kong, 72 consecutive patients with IgA nephropathy presenting as hematuria and minimal proteinuria $(0.4 \mathrm{~g} /$ day or less) were followed for a median of 84 months; $33 \%$ developed proteinuria, $26 \%$ became hypertensive, and $7 \%$ developed impaired kidney function. ${ }^{176}$ The presence of hematuria and glomerular IgA deposition is associated with increased risk of progressive kidney disease even in the absence of other clinical findings. ${ }^{175}$ For this reason, there is broad consensus that people with known $\operatorname{IgA}$ nephropathy should not donate a kidney. Examination of a series of 510 implantation biopsies at a large center in Tokyo (including 446 living donors) found latent mesangial IgA deposition to be relatively common in healthy Japanese living donors, present in $16.1 \%$ of living donor allograft biopsies. ${ }^{177}$ Mesangial IgA deposition was associated with a mild degree of microhematuria, mesangial proliferation and glomerular macrophage infiltration in some of the affected individuals, especially with C3 deposition.

Persistent hematuria without defined renal histopathology has been associated with proteinuria after kidney donation. In a series of 242 living kidney donors at one center in Japan, persistent predonation hematuria was identified in $8.3 \%(18.6 \%$ vs $6 \%$ in those with vs without family history of IgA nephropathy or Alport syndrome). ${ }^{178} 95 \%$ of those with persistent predonation hematuria continued to have persistent hematuria after donation over median 27 month follow-up (compared with $28 \%$ of those with predonation occasional hematuria and $5 \%$ without predonation hematuria). Predonation hematuria was associated with increased likelihood of persistent proteinuria (dipstick $\geq 1+$ ) after donation (without dysmorphic red blood cells: adjusted OR 3.8; with dysmorphic red blood cells: adjusted OR 12.3). Predonation hematuria was not associated with postdonation GFR, but persistent postdonation hematuria with dysmorphic red blood cells was associated with significant GFR decline over the study period.

\section{What Prior Guidelines Recommend}

Previous guidelines recommend that evaluation of donor candidates for causes of hematuria include urine culture and imaging, ${ }^{48}$ cystoscopy if older than 40 years, ${ }^{48}$ urine cytology and "complete" urological evaluation. ${ }^{54} \mathrm{~A}$ recent Canadian protocol recommends tests of urine culture, urine cytology, 24-hour urine calcium, metabolic stone workup, and then if the cause of hematuria is undetermined, cystoscopy and a native kidney biopsy. ${ }^{179}$ In the absence of an identified cause, evaluation by kidney biopsy has been advised if hematuria is $>1+{ }^{48}$ or possibly caused by glomerular disease ${ }^{38,54}$

While a number of prior living donor guidelines recommend kidney biopsy as part of the evaluation of persistent microhematuria before donation, ${ }^{48,180}$ few articulate criteria for donor selection. The 2011 British Transplantation Society guidelines offer a "moderate quality" recommendation that "glomerular pathology precludes donation, with the possible exception of thin basement membrane disease." ${ }^{48}$ Canadian Blood Service's protocol for KPD defines IgA nephropathy and Alport syndrome (including carrier status) as exclusions to donation. ${ }^{179}$

The 2013 "Expert Guidelines for the Management of Alport Syndrome and Thin Basement Membrane Nephropathy" include several consensus-based recommendations related to living donation selection ${ }^{181}$ : A) "Individuals with TBMN may be kidney donors if they have normal BP, proteinuria, and renal function" and if a biopsy is done and Alport syndrome is excluded." Close monitoring and use of nephroprotective strategies are advised; B) "Individuals from families with autosomal recessive Alport syndrome who have only one of the causative mutations (parents, offspring, some siblings) may be kidney donors if they have normal BP, proteinuria levels, and renal function; if coincidental kidney disease has been excluded by kidney biopsy; and if X-linked Alport syndrome has been excluded by genetic testing." The document recommends "discouraging affected mothers of males with X-linked 
Alport syndrome from renal donation because of their own risk of kidney failure."

\section{RESEARCH RECOMMENDATIONS}

- Perform long-term follow-up studies of living donors with infrequent clinical conditions including:

- Alport female "carrier" state

- TBMN

Incidental IgA deposition in glomeruli and isolated hematuria

Nephrolithiasis

- These studies should include appropriate controls and examine endpoints including CKD and kidney failure, premature death, and health-related quality of life.

\section{CHAPTER 8: KIDNEY STONES}

The ERT search parameters did not identify evidence from eligible studies pertinent to the recommendations in chapter 8 and therefore the following recommendations are "Not Graded."

\section{Evaluation}

8.1: Donor candidates should be asked about prior kidney stones, and related medical records should be reviewed if available.

8.2: The imaging performed to assess anatomy before donor nephrectomy (eg, computed tomography angiogram) should be reviewed for the presence of kidney stones.

8.3: Donor candidates with prior or current kidney stones should be assessed for an underlying cause.

\section{Selection}

8.4: The acceptance of a donor candidate with prior or current kidney stones should be based on an assessment of stone recurrence risk and knowledge of the possible consequences of kidney stones after donation.

\section{Counseling}

8.5: Donor candidates and donors with current or prior kidney stones should follow general population, evidencebased guidelines for the prevention of recurrent stones.

\section{RATIONALE}

\section{Evaluation}

Transplant programs typically assess donor candidates with past or present, symptomatic or asymptomatic kidney stones. In a cohort of approximately 2000 living kidney donor candidates who underwent computed tomography (CT) angiograms/urograms at a large US center (mean age, 43 years; $92 \%$ white; $58 \%$ women), $3 \%$ of candidates had past symptomatic kidney stones, and $11 \%$ had evidence of stones on renal imaging. ${ }^{182}$

A kidney stone in an individual with a solitary kidney can potentially obstruct the ureter, leading to acute kidney injury, and may result in urgent hospital attention and even surgical intervention. Donor candidates should have a detailed evaluation including a careful history and medical record review of any prior or current kidney stones. In the case of a prior history of stones, additional details that should be assessed include the time and location of prior episodes, and any prior investigations or treatments. The frequency of kidney stones varies by sex, race and climate. The overall prevalence of kidney stones is approximately 6 to $9 \%$ in men, and 3 to $4 \%$ in women, and most stones are composed of calcium oxalate. ${ }^{183}$ Living kidney donors without a predonation history of kidney stones have no difference in the risk of developing kidney stones after donation, or in receiving a urologic procedure for kidney stones after donation, compared with selected nondonors matched for similar baseline health. ${ }^{184}$

\section{Asymptomatic Kidney Stones Seen on Imaging}

Approximately $5 \%$ of individuals have evidence of an asymptomatic kidney stone on CT angiography performed as part of the donor evaluation. ${ }^{185}$ Such CT scans may detect very small calcifications in the kidneys in patients who are asymptomatic and have no history of a clinically recognized kidney stone; very small 1 to $2 \mathrm{~mm}$ calcifications in the renal papillae found on CT scans are referred to as Randall's plaques. These plaques have uncertain prognostic significance.

\section{Evaluation of Donor Candidates and Donors with Prior or Current Kidney Stones}

Donor candidates and donors with current or prior kidney stones should have an evidence-based evaluation of nephrolithiasis. For example, the 2014 American Urological Association Guideline provides comprehensive consensus and evidencebased recommendations for the evaluation of adult patients with kidney stones ${ }^{186}$ :

1. A clinician should perform a screening evaluation consisting of a detailed medical and dietary history, serum chemistries and urinalysis on a patient newly diagnosed with kidney or ureteral stones. (clinical principle)

2. Serum intact parathyroid hormone (PTH) concentration should be obtained as part of the screening evaluation if primary hyperparathyroidism is suspected. (clinical principle)

3. When a stone is available, a stone analysis should be performed at least once. (clinical principle)

4. Clinicians should obtain or review available imaging studies to quantify stone burden. (clinical principle)

5. Additional metabolic testing should be performed in high-risk or interested first-time stone formers and recurrent stone formers. (moderate evidence)

6. Metabolic testing should consist of one or two 24-hour urine collections obtained on a random diet and analyzed at minimum for total volume, $\mathrm{pH}$, calcium, oxalate, uric acid, citrate, sodium, potassium and creatinine. (expert opinion)

Similarly, the 2016 European Association of Urology guideline also provides comprehensive expert and evidence-based recommendations on the evaluation of adults with kidney stones, ${ }^{187}$ as do the Caring for Australians with Renal Impairment (CARI) guidelines. ${ }^{183}$

\section{Risk Factors for Recurrent Stones}

Prospective studies have shown the median recurrence rate of kidney stones is 15 per 100 person-years. ${ }^{188}$ However, the risk of recurrence after a single stone is difficult to predict in an individual. Compared with older adults, younger adults 
have more remaining years to live, and so have a higher lifetime chance of kidney stone recurrence.

Characteristics associated with a higher lifetime risk of stone recurrence include ${ }^{183}$ :

- Younger age ( $<40$ years)

- A family history of kidney stones

- Frequent, recurrent kidney stones

Characteristics associated with a lower lifetime risk of stone recurrence include:

- Older age ( $\geq 40$ years)

- No prior symptoms of kidney stones

- A kidney stone that is less than $15 \mathrm{~mm}$, solitary and unilateral

\section{Selection and Management}

The acceptance of a donor candidate with prior or current kidney stones should be based on an assessment of stone recurrence risk and knowledge of the possible consequences of kidney stones after donation.

As recommended in chapter 5 (Predonation Kidney Function), when asymmetry in GFR, parenchymal abnormalities (including stones), vascular abnormalities, or urological abnormalities are present but do not preclude donation, the more affected kidney should be used for donation.

\section{Possible Consequences of Kidney Stones}

Kidney stones may be associated with a higher risk of CKD and ESKD, with possible mechanisms including obstructive uropathy or pyelonephritis, crystal plugs at tips of the renal papilla and parenchymal injury from treatments such as shockwave lithotripsy. ${ }^{183,189}$ One or more episodes of kidney stones were associated with a 2-fold higher risk of ESKD in one population-based study from Alberta, Canada. ${ }^{190}$ The association was stronger in patients with 2 or more episodes of kidney stones versus a single episode of kidney stones. A National Health and Nutrition Examination Survey also reported that a history of kidney stones was associated with CKD and ESKD, however the effect was largely confined to women. ${ }^{191}$ Among 10678 patients in the Atherosclerosis Risk in Communities study, nephrolithiasis history was associated with a $29 \%\left(\mathrm{HR},{ }_{1.07} 1.29_{1.54}\right)$ higher risk of CKD in demographic-adjusted analyses, but the association was no longer statistically significant after multivariable adjustment (HR, $\left.0.901 .09_{1.32} ;\right)^{1}{ }^{192}$ To inform this guideline, CKD-PC analyzed multiple cohorts (see chapter 1). ${ }^{7}$ They found the association between a prior history of kidney stones and ESKD was not significant in the meta-analysis; for this reason a history of kidney stones does not appear as a characteristic in the online tool to predict the 15 -year and lifetime chance of kidney failure in the absence of donation. ${ }^{7}$

\section{Ex-vivo Removal of Kidney Stones}

There are reports of nephrolithiasis-related adverse events for recipients of an allograft with a stone left in situ. ${ }^{193}$ There are also reports on the safety and success of ex vivo ureteroscopy to remove stones from explanted donor kidneys before transplantation. ${ }^{185}$

\section{Counseling}

Donors and donor candidates who develop kidney stones should follow general population evidence-based guidelines for dietary and pharmacologic-based strategies to prevent recurrent kidney stones. Recent systematic reviews and clinical practice guidelines summarize this evidence. ${ }^{194,195}$ For example, there is low-strength evidence that increased fluid intake to achieve a urine output of at least 2 liters per day (vs normal fluid intake) and reduced soft-drink consumption lowers the risk of recurrent stones. In patients with multiple past calcium stones, most of whom increased their fluid intake, there is moderate-strength evidence that thiazide diuretics, citrate supplements, and allopurinol each further reduce the risk of future stones compared with placebo or control, although the benefit from allopurinol seemed limited to patients with baseline hyperuricemia or hyperuricosuria.

\section{What Prior Guidelines Recommend}

Several prior guidelines and US policy requirements describe evaluation methods and/or acceptance criteria for donor candidates with prior or current kidney stones. ${ }^{38,48,51,55,179}$ Generally, most prior guidelines recommend complete metabolic testing (serum PTH, 24-urine collection for calcium, phosphate, oxalate, citrate, urate, creatinine and sodium), and one guideline recommends all such candidates be assessed by a urologist. ${ }^{179}$ Several prior guidelines recommend the current presence of bilateral kidney stones, multiple stones, or nephrocalcinosis precludes donation, while a current small single stone may be eligible to proceed with donation. ${ }^{38,54,55,179,196}$

\section{RESEARCH RECOMMENDATIONS}

- Perform follow-up studies of approved donors with current stones, risk factors for recurrent stones, or small calcifications discovered on imaging to determine impact of these factors on long-term outcomes compared to donors without these characteristics, and also compared to nondonors with similar risk factors.

- Develop a tool to estimate the risk of postdonation kidney stones according to predonation clinical and demographic factors.

\section{CHAPTER 9: HYPERURICEMIA, GOUT, AND MINERAL AND BONE DISEASE}

The ERT search parameters did not identify evidence from eligible studies pertinent to the recommendations in chapter 9 and therefore the following recommendations are "Not Graded.”

\section{Evaluation}

9.1: Donor candidates should be asked about prior episodes of gout.

\section{Counseling}

9.2: Donor candidates may be informed that donation is associated with an increase in serum uric acid concentration, which may increase the risk for gout.

9.3: Donor candidates and donors with prior episodes of gout should be informed of recommended methods to reduce their risk of future episodes of gout.

\section{RATIONALE}

\section{Hyperuricemia and Gout}

High serum uric acid concentration is a potent risk factor for gout, for which the 10-year incidence is estimated to be 
$1 \%, 5 \%$, and $49 \%$ for uric acid levels of $<7.0,7.0-8.9$, and $\geq 9.0 \mathrm{mg} / \mathrm{L}$ respectively. ${ }^{197,198}$ A decline in GFR results in less uric acid excretion and a higher serum uric acid concentration. ${ }^{199,200}$ These changes are evident with the $25 \%$ to $40 \%$ reduction in GFR that occurs after living kidney donation. ${ }^{139,201,202}$ For example, one prospective US cohort study found that at 6 months after nephrectomy, donors versus healthy nondonor controls had $8.2 \%$ higher serum uric acid level [mean values of $5.3 \pm 1.1 \mathrm{mg} / \mathrm{dL}(315.2 \pm 65.4 \mu \mathrm{mol} / \mathrm{L}$; standard deviation) vs $4.9 \pm 1.2 \mathrm{mg} / \mathrm{dL}(291.5 \pm 71.4 \mu \mathrm{mol} / \mathrm{L})$; $P<0.001$ ], that persisted over 36 months of follow-up. ${ }^{139,201}$ Another study found donors to have $20 \%$ higher serum uric acid concentrations at a mean of 7 years after nephrectomy compared with predonation. ${ }^{203}$

In a study of 1988 living kidney donors from Ontario, Canada, who were followed for a median of 8.8 years (maximum, 20.8 years), living kidney donors were more likely to be diagnosed with gout compared to matched nondonors with similar baseline health, although the absolute risk remained low (3.5 vs 2.1 events per 1000 person-years; HR $\left.1.21 .6_{2.1} ;\right)^{203}$ At 8 years, the cumulative incidence of gout was $1.4 \%$ higher in donors compared to nondonors $(3.4 \%$ vs $2.0 \%){ }^{203}$ This report was not reviewed by the ERT but would be expected to have a moderate risk of bias similar to other reported outcomes from this cohort. Based on this report, some transplant teams may decide to inform donor candidates that donation is associated with an increase in serum uric acid concentration, which may increase the risk of gout. However, the authors of the primary report suggested the finding first be corroborated in other studies. In this guideline we endorse that donor candidates be asked about prior gout episodes, but given the limitations of existing information, we did not propose measuring uric acid as a standard part of evaluation or follow-up processes at this time. The risk of gout after kidney donation may vary based on demographic characteristics. A study of 4650 kidney donors from the US including 13.1\% African Americans, found that, by 7 years, African Americans were almost twice as likely to develop gout as white donors $\left(4.4 \%\right.$ vs $2.4 \%$; adjusted HR, 1.0 1.83.2). ${ }^{204}$ Postdonation gout risk also increased with older age at donation and was nearly 3-times higher in men compared with women. ${ }^{204}$

In nondonor populations hyperuricemia is associated with a higher risk of CVD and new-onset CKD. ${ }^{203,205}$ However, the prognostic significance of hyperuricemia in living kidney donors is currently uncertain and warrants additional study. In the US cohort study mentioned above, compared with matched donors without gout, donors with gout had more frequent diagnoses of acute kidney injury, CKD, and other disorders of the kidney.

Donor candidates and donors with prior episodes of gout should be informed of recommended methods to reduce their risk of future episodes of gout. Previous clinical practice guidelines cite modest to low evidence for certain diet and lifestyle measures (eg, avoiding excessive alcohol, and avoiding ingestion of organ meats high in purine content) and possibly pharmacological therapies. ${ }^{206}$ The prevention and treatment of hyperuricemia and gout may be complicated by decreased kidney function. The dose of allopurinol, which is frequently used to treat increased serum uric acid and prevent gout, should be adjusted in patients with decreased kidney function. Dose reductions may not be necessary with newer uricosuric agents. ${ }^{207}$ Nonsteroidal anti-inflammatory drugs should probably be used cautiously in patients with decreased kidney function including living kidney donors. The treatment of choice for acute gout is colchicine, however the dose of colchicine should be reduced in patients with decreased kidney function.

\section{Metabolic Bone Disease}

The effect of the modest decrease in GFR on the development of metabolic bone disease among kidney donors is not clear. Several recent studies describe changes in measures of bone mineral metabolism in kidney donors. These changes include a decline in the serum concentration of 1,25-dihydroxyvitamin D and phosphate, a decline in tubular phosphate reabsorption, and a rise in the concentration of serum PTH. ${ }^{139,201,202,208}$ For example, one study showed that kidney donors had higher serum concentrations of fibroblast growth factor-23 and greater fractional excretion of inorganic phosphate compared with nondonor controls. ${ }^{209}$ The Assessing Long Term Outcomes of Living Donation prospective cohort study found a $23 \%$ higher PTH concentration among 206 donors assessed at 6 months postdonation compared with concentrations among 198 healthy controls ( $52.7 \mathrm{vs} 42.8 \mathrm{pg} / \mathrm{mL}$, respectively $)^{201}$ that persisted at 36-month follow-up. ${ }^{139}$ Similarly, the Chronic Renal Impairment in Birmingham study of 68 donors at 2 United Kingdom centers reported larger increases in serum fibroblast growth factor-23 and parathyroid hormone concentrations compared with prospective changes in these parameters among healthy nondonors. ${ }^{202}$

The long-term effects of these changes on the risk of fractures, CVD, or other outcomes important to donors is uncertain, therefore the WG made no recommendations for managing mineral and bone disorders that would differ from guidelines for the general population. The ERT identified a single study of fractures in kidney donors (moderate risk of bias, with only a single study the quality of evidence was deemed very low; Evidence Report Table 8, SDC, http://links.lww.com/TP/B434; Supplemental Appendix Table D5 SDC, http://links.lww.com/ TP/B432). In this study, 2015 kidney donors in Ontario, Canada, experienced no increase in the risk of non-traumarelated upper- or lower-extremity fractures compared with healthy nondonors. 210

\section{What Prior Guidelines Recommend}

As many of the studies on hyperuricemia, gout and mineral and bone disease in living kidney donors are recent, these issues have generally not been addressed in prior donor guidelines. The British Transplantation Society describes the importance assessing a history of gout as part of the candidate evaluation. ${ }^{48}$ Amsterdam Forum and Spanish Society of Nephrology and Spanish National Transplant Organisation guidelines describe serum uric acid as a standard test in the evaluation of all living kidney donor candidates. ${ }^{38,54}$

\section{RESEARCH RECOMIMENDATIONS}

- Determine the effects of hyperuricemia in living kidney donor candidates and donors on the incidence of gout and CVD.

- Perform long-term follow-up of donors and healthy nondonor controls to determine the impact of kidney donation on gout recurrence.

- Perform long-term follow-up of donors and healthy nondonor controls to determine the impact of kidney donation (if any) on fracture risk. 


\section{CHAPTER 10: PREDONATION BLOOD PRESSURE}

Except in the case of Recommendation 10.6, the ERT search parameters did not identify evidence from eligible studies pertinent to the recommendations in chapter 10 and therefore the following recommendations are "Not Graded."

\section{Evaluation}

10.1: Blood pressure should be measured before donation on at least 2 occasions by clinical staff trained in accurate measurement technique, using equipment calibrated for accuracy.

10.2: When the presence or absence of hypertension in a donor candidate is indeterminate based on history and clinic measurements (eg, blood pressure is high normal or variable), blood pressure should be further evaluated using ambulatory blood pressure monitoring (ABPM) or repeated using standardized blood pressure measurements.

\section{Selection}

10.3: Normal blood pressure, as defined by guidelines for the general population in the country or region where donation is planned, is acceptable for donation.

10.4: Donor candidates with hypertension that can be controlled to systolic blood pressure less than $140 \mathrm{~mm} \mathrm{Hg}$ and diastolic blood pressure less than $90 \mathrm{~mm} \mathrm{Hg}$ using 1 or 2 antihypertensive agents, who do not have evidence of target organ damage, may be acceptable for donation. The decision to approve donor candidates with hypertension should be individualized based on demographic and health profile in relation to the transplant program's acceptable risk threshold.

\section{Counseling}

10.5: Donor candidates should be counseled on lifestyle interventions to address modifiable risk factors for hypertension and cardiovascular disease, including healthy diet, smoking abstinence, achievement of healthy body weight, and regular exercise according to guidelines for the general population. These measures should be initiated before donation and maintained lifelong.

10.6: We suggest that donor candidates should be informed that blood pressure may rise with aging, and that donation may accelerate a rise in blood pressure and need for antihypertensive treatment over expectations with normal aging. (2D)

\section{RATIONALE}

\section{Evaluation and Definitions}

\section{BP Measurement and Classification}

It is important to use an accurate, well-calibrated device and an appropriately sized BP cuff based on arm length and circumference. An overly small cuff will overestimate and an excessively large cuff will underestimate true BP levels. The donor candidate should be seated quietly with back supported, feet on the floor and arm supported at heart level for the measurements. It is advisable to measure BP on at least 2 separate occasions by trained staff, or on one occasion plus ambulatory $\mathrm{BP}$ monitoring (ABPM) to minimize anxiety effects in donor candidates. ${ }^{21}$ Automated serial BP measurements can also eliminate a "white coat" effect. ${ }^{212}$ Multiple prior guidelines and policies for the evaluation and care of living kidney donor candidates recommend assessment of predonation BP on several occasions, ${ }^{212,213}$ and/or consideration of ABPM in donor candidates with elevated office readings, receiving antihypertensive therapy, or who are older at evaluation. $38,50,51,196,214,215$

Hypertension is defined by office readings of systolic SBP of $140 \mathrm{~mm} \mathrm{Hg}$ or greater or diastolic BP (DBP) of $90 \mathrm{~mm}$ $\mathrm{Hg}$ or greater, out-of-office daytime mean ABPM or home measurements of SBP of $135 \mathrm{~mm} \mathrm{Hg}$ or greater or DBP of $85 \mathrm{~mm} \mathrm{Hg}$ or greater, ${ }^{213}$ or the need for medication to control BP. Note that some antihypertensive agents may be used for indications other than BP treatment (eg, diuretics for edema or beta-blockers for migraine headache prevention), and that the indication for the prescribed medications should be determined as part of the evaluation.

White coat hypertension is defined as hypertension by office BP measurements with normal out of office measurements by ABPM or home readings. Individuals with white coat hypertension have lower cardiovascular risk than persons with hypertension, but may carry increased risk for future hypertension. ${ }^{212,216}$ Population-based studies suggest that $20-25 \%$ of adults may have white coat hypertension. ${ }^{216}$ Individuals with treated hypertension may also have a "white coat effect," such that elevated BP is recorded in the medical environment even when treated BP is controlled by ABPM or home readings.

Masked hypertension is defined as normal BP by office measurements with hypertension by ABPM or home readings. Like sustained hypertension, masked hypertension is accompanied by increased risk for hypertensive target organ damage, and thus treatment is warranted if identified. Population-based studies suggest that $10-30 \%$ of adults may have masked hypertension. ${ }^{216,217}$ Masked hypertension cannot be identified in donor candidates in whom $\mathrm{BP}$ is measured by office readings alone, but the implications of requiring $\mathrm{ABPM}$ or home readings to screen for masked hypertension in donor candidates (eg, outcomes, cost, efficiency) are not defined.

\section{Hypertension Risk Factors and Counseling}

Potentially modifiable risk factors for hypertension include use of certain medications (eg, nonsteroidal anti-inflammatory agents, decongestants, stimulants) and presence of certain lifestyle factors (eg, excess alcohol intake, high sodium diet, use of dietary supplements, or smoking). ${ }^{218-220}$ Nonmodifiable hypertension risk factors include a family history of hypertension, race, and age. Additional considerations include women with a history of preeclampsia or gestational hypertension. In this setting we suggest predonation counseling on the potential for increased cardiovascular risk and emphasis on healthy behaviors to reduce cardiovascular risk. Risk factors for hypertension by themselves do not constitute contraindications to donation in a normotensive person. Consistent with recommendations for the general population, ${ }^{221}$ British Transplantation Society and Renal Association guidelines ${ }^{48}$ recommend lifestyle measures in kidney donors to reduce the risk of hypertension and its consequences, including frequent exercise, smoking cessation, and weight loss where appropriate.

Target organ damage may be manifest as prior occurrence of a cardiovascular event such as myocardial infarction or stroke, urine AER greater than $30 \mathrm{mg} / \mathrm{d}$ (ACR > $30 \mathrm{mg} / \mathrm{g}$ or 
$3 \mathrm{mg} / \mathrm{mmol}$ ), reduced kidney function $(\mathrm{eg}, \mathrm{GFR}<60 \mathrm{~mL} / \mathrm{min}$ per $1.73 \mathrm{~m}^{2}$ ), hypertensive retinopathy, and/or evidence of left ventricular hypertrophy. ${ }^{213}$

Lifestyle modification can effectively treat hypertension without medication or with fewer medications and lower dosages required to achieve BP control. ${ }^{221}$ Lifestyle modifications include healthy diet, smoking cessation, weight loss if overweight, regular exercise, and discontinuation of potential contributing medications according to guidelines for the general population. Follow-up of patents with hypertension is critical for monitoring of control in relation to targets and for monitoring and management of complications. The importance of access to healthcare and regular follow-up have also been emphasized in the selection and care of hypertensive donor candidates in 2 prior guidelines, ${ }^{38,54}$ whereas the Spanish Society of Nephrology and Spanish National Transplant Organisation guidelines specify "reasonable guarantee that the donor will follow the check-up period and treatment indefinitely" among the criteria for acceptance of a hypertensive donor candidate. ${ }^{54}$

\section{Selection}

Impact of Reduced GFR on BP in the General Population and After Donation

Reduced kidney function may cause or worsen hypertension in the general population. ${ }^{22}$ While it is documented that BP often rises with aging, ${ }^{223}$ GFR reduction from kidney donation may accelerate the risk or progression of hypertension over time to a greater extent than expected from normal aging, possibly due to physiological alterations (eg, hyperfiltration in the remaining kidney, changes in vascular tone and reninangiotensin-aldosterone regulation) and/or heightened detection at donor follow-up. ${ }^{224,225}$ Existing retrospective studies examining the impact of kidney donation on hypertension risk have been limited by short follow-up times, high rates of loss to follow-up, lack of controls and/or comparisons to unselected general rather than healthy populations. Use of antihypertensive medications was lower in a cohort of privately-insured donors compared with age- and sexmatched unscreened beneficiaries in the same insurance plan. ${ }^{226}$ In contrast, a systematic review including data for 5145 predominantly white donors estimated $6 \mathrm{~mm} \mathrm{Hg}$ higher weighted mean SBP and $4 \mathrm{~mm} \mathrm{Hg}$ higher weighted mean DBP in donors compared with controls after an average of 7 years $^{224}$ (Supplemental Appendix Tables D1 and D2, SDC, http://links.lww.com/TP/B432). An administrative claims linkage study of 1278 (primarily white race) living donors in Ontario, Canada followed for a mean of 6 years (range, 1-16 years) found a higher incidence of claims-based hypertension diagnoses ( $16.3 \%$ vs $11.9 \%$; HR, $\left.1.21 .4_{1.7}\right)$ among living donors compared with matched controls who were also screened for the absence of baseline comorbidities through administrative claims. ${ }^{225}$

Among more recent donor cohorts, higher rates of postdonation hypertension and antihypertensive medication use in African American compared with white donors have been reported. ${ }^{226-228}$ While these patterns parallel hypertension prevalence differences in the general population, ${ }^{228}$ one small study found higher rates of postdonation hypertension among 103 African American donors compared with racematched "healthy" nondonor controls (41\% vs $18 \%$ at an average of 6.8 years postdonation) ${ }^{229}$ (Supplemental Appendix
Table D10, SDC, http://links.lww.com/TP/B432). Furthermore, many donors in this study were unaware of their hypertension. Associations of age at donation and sex with postdonation hypertension have been inconsistent (Supplemental Appendix Tables D7 and D9, SDC, http://links.lww. com/TP/B432).

Based on these data, we suggest that donor candidates should be informed that BP may rise with aging, and that donation may accelerate the rise in BP and the need for antihypertensive treatment over that expected with normal aging, especially if $\mathrm{BP}$ is high-normal before donation and among African American donor candidates. Furthermore, antihypertensive medication is more likely to be prescribed after donation.

\section{Hypertension as a Cause of CKD in the General Population}

Hypertension is a contributing cause of CKD in the general population, although in many cases, CKD may be the cause of hypertension. A recent meta-analysis based on data from nearly 5 million healthy persons identified from 7 general population cohorts found that every $20 \mathrm{~mm} \mathrm{Hg}$ increase in SBP was associated with a $42 \%$ increase (adjusted HR, $\left.1.271 .42_{1.58}\right)$ in the risk of ESKD over median cohort follow-up of 4 to 16 years. Use of antihypertensive medications was also associated with increased ESKD risk (adjusted HR, ${ }_{1.01} 1.35_{1.82}$ over cohort follow-up). ${ }^{7}$ After calibration to annual ESKD incidence in the US healthy population, variations in the projected 15-year and lifetime risks of ESKD based on level of SBP from this analysis were generated according to age, sex, and race for healthy persons (assuming age-specific GFR, urine ACR $4 \mathrm{mg} / \mathrm{g}$ [0.4 mg/mmol], BMI $26 \mathrm{~kg} / \mathrm{m}^{2}$, and absence of diabetes mellitus; (Figures 14 and 15). While these figures are useful for visualizing the impact of higher SBP on increased ESKD risk, we endorse consideration of BP as part of the assessment of predicted long-term ESKD risk based on a donor candidate's complete demographic and health profile (as opposed to consideration of single risk factors in isolation).

Clinical consequences of hypertension vary by race in the general population. Treating mild-to-moderate primary hypertension may not halt kidney disease progression in nondiabetic African Americans, whereas hypertension control appears to slow kidney disease progression in European Americans. ${ }^{230-234}$ Recent literature supports that at least a portion of kidney failure previously attributed to hypertensive nephrosclerosis in persons of African descent may be genetically mediated by coding variants in the gene for APOL1 and not modifiable by antihypertensive therapy (see chapter 14). ${ }^{235-238}$

\section{Predonation Hypertension as a Risk Factor for Adverse Outcomes after Kidney Donation}

Living donor candidates undergo a rigorous evaluation process which includes measurement of kidney function and urine protein, but subtle scarring of the kidney from hypertensive nephrosclerosis may not be detected by these tests. ${ }^{239}$ Compensatory hyperfiltration in the remaining kidney is normal after nephrectomy ${ }^{240,241}$; however, subclinical pathology or aging processes may impair compensation and reduce postdonation GFR. ${ }^{240,242}$

The ERT identified 3 studies, rated as very low quality in reporting postdonation outcomes according to predonation $\mathrm{BP}$ or hypertension status (Evidence Report Table 19, SDC, 

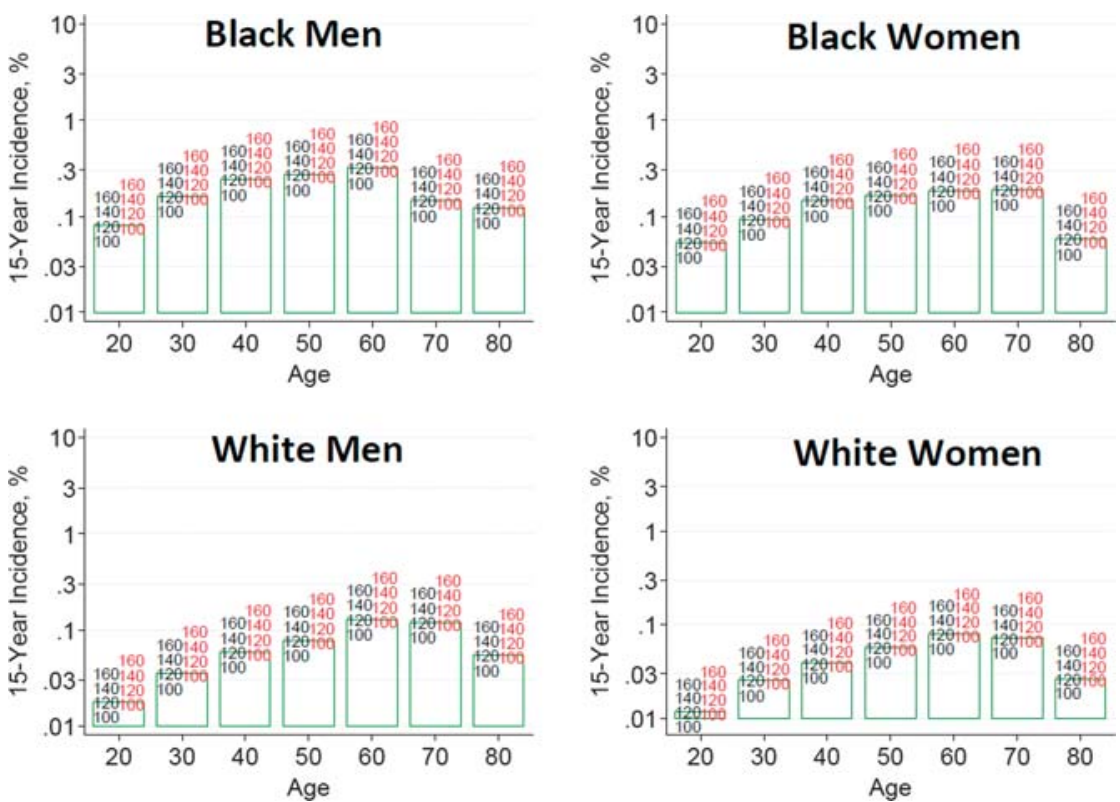

FIGURE 14. Estimated 15-year incidence (\%) of ESKD in the United States according to baseline systolic blood pressure (SBP) and demographic profile from the CKD-PC. SBP $\mathrm{mm} \mathrm{Hg}$ without (black) and with (red) antihypertension medication. *The base-case scenario is defined as: age-specific eGFR (114, 106, 98, 90, 82, 74, and $66 \mathrm{~mL} / \mathrm{min}$ per $1.73 \mathrm{~m}^{2}$ for ages 20, 30, 40, 50, 60, 70, and 80 years, respectively), SBP $120 \mathrm{~mm} \mathrm{Hg}$, urine ACR $4 \mathrm{mg} / \mathrm{g}[0.4 \mathrm{mg} / \mathrm{mmol}]$, BMl $26 \mathrm{~kg} / \mathrm{m}^{2}$, and no diabetes mellitus or antihypertensive medication use. These were selected as being representative of recent US living kidney donors where, with the exception of eGFR, there was little variation in health characteristics by age. ACR, albumin-to-creatinine ratio; BMI, body mass index; CKD-PC, Chronic Kidney Disease-Prognosis Consortium, eGFR, estimated glomerular filtration rate; ESKD, end-stage kidney disease. Reprinted from Grams ME, Sang Y, Levey AS, et al. Kidney-failure risk projection for the living kidney-donor candidate. N Engl J Med. 2016;374:411-421.

http://links.lww.com/TP/B434, and Supplemental Appendix Table D16, SDC, http://links.lww.com/TP/B432). In a combined cohort of donors and matched healthy nondonors in
Norway, each $1 \mathrm{~mm} \mathrm{Hg}$ increase in SBP was associated with a small increase in cardiovascular death and ESKD during up to 25 years of follow-up. ${ }^{32}$ A study of only 6 hypertensive donors
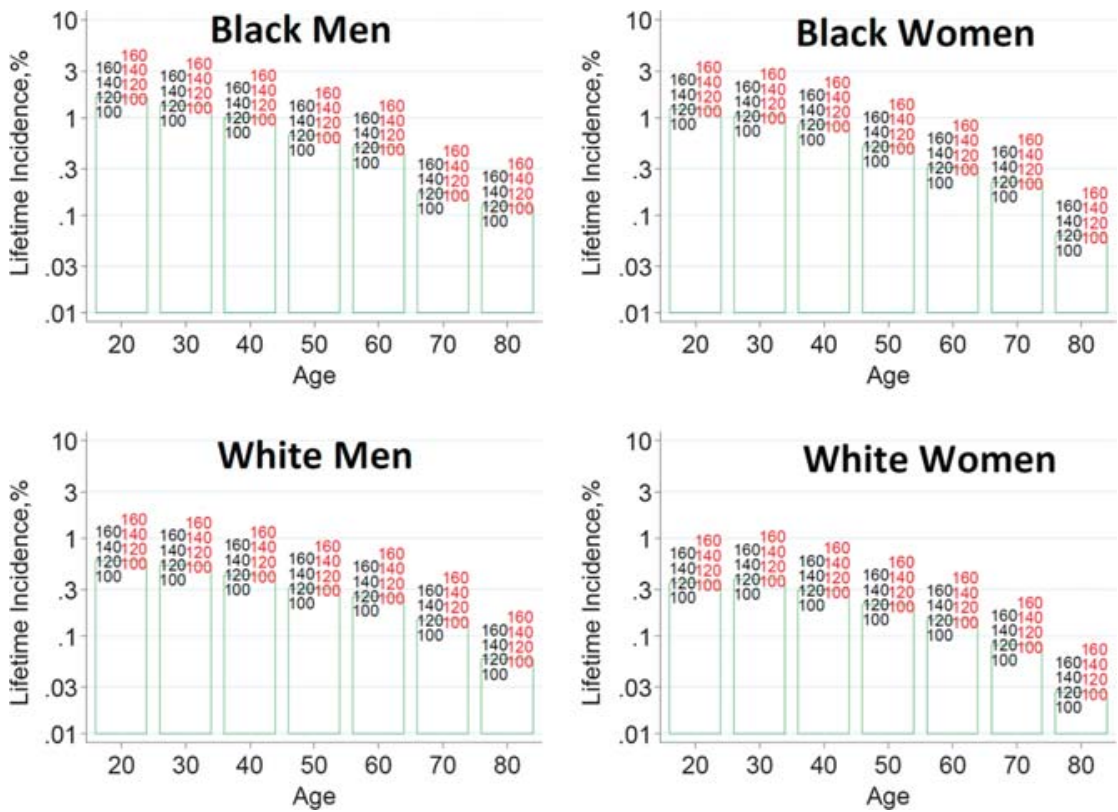

FIGURE 15. Estimated lifetime incidence (\%) of ESKD in the United States according to baseline systolic blood pressure (SBP) and demographic profile from the CKD-PC. SBP $\mathrm{mm} \mathrm{Hg}$ without (black) and with (red) antihypertension medication. ${ }^{*}$ The base-case scenario is defined as: age-specific eGFR $\left(114,106,98,90,82,74\right.$, and $66 \mathrm{~mL} / \mathrm{min}$ per $1.73 \mathrm{~m}^{2}$ for ages 20, 30, 40, 50, 60, 70, and 80 years, respectively), SBP $120 \mathrm{~mm} \mathrm{Hg}$, urine ACR $4 \mathrm{mg} / \mathrm{g}[0.4 \mathrm{mg} / \mathrm{mmol}]$, BMl $26 \mathrm{~kg} / \mathrm{m}^{2}$, and no diabetes mellitus or antihypertensive medication use. These were selected as being representative of recent US living kidney donors where, with the exception of eGFR, there was little variation in health characteristics by age. Lifetime risk projections are based on 15 years of follow-up data and calibrated to the incidence of ESKD in the low-risk population, and thus are likely imprecise. ACR, albumin-to-creatinine ratio; BMl, body mass index; CKD-PC, Chronic Kidney Disease-Prognosis Consortium, eGFR, estimated glomerular filtration rate; ESKD, end-stage kidney disease. Reprinted from Grams ME, Sang Y, Levey AS, et al. Kidney-failure risk projection for the living kidney-donor candidate. N Engl J Med. 2016;374:411-421.7 
reported CKD in $4(67 \%)$ compared with $22 \%$ of nonhypertensive donors during an average of 5.4 years followup. ${ }^{243}$ A third study of 16 hypertensive donors was deemed at high risk of bias. ${ }^{244} \mathrm{~A}$ systematic review by Young et al concluded that results from this and 2 additional studies were substantially heterogeneous and thus results were not pooled. ${ }^{26}$

Additional studies that did not meet criteria for the ERT evidence review include a large study based on linkage of the US Transplant Registry with national death records that found higher perioperative mortality among donors with versus without predonation hypertension (36.7 vs 1.3 per 10 000). ${ }^{23}$ While reported baseline hypertension was not associated with long-term mortality, SBP of $140 \mathrm{~mm} \mathrm{Hg}$ or greater at donor registration was associated with 3-times the adjusted RR of death over 12 years compared to SBP less than $120 \mathrm{~mm} \mathrm{Hg}$ (adjusted HR 1.13.39.7). A single-center study of 24 white, older (mean age, 53 years) donors with predonation hypertension (awake ABPM > 135/85 mm Hg and clinic/nurse-measured $\mathrm{BP}>140 / 90 \mathrm{~mm} \mathrm{Hg}$ ) that was not included in the evidence review based on sample size found similar postdonation GFR reduction and urine protein excretion as in normotensive donors, and no increase in urinary protein excretion compared with predonation values over a mean 282 days of follow-up. ${ }^{245}$ A recent US registry study found that ESKD among donors was more likely to be attributed to hypertension if the duration of follow-up was more than 10 years compared with less than 10 years. ${ }^{31}$

The WG concluded that the decision to approve donation in candidates with hypertension should be individualized based on demographic and health profile in relation to the transplant program's acceptable risk threshold. Donor candidates with hypertension should be treated according to guidelines for the general population in the country or region where donation will occur. BP control should be confirmed by standardized BP measurements over at least several weeks before donation. Donor candidates with hypertension should be informed that donation may accelerate the rise in BP and the need for antihypertensive treatment over expectations with normal aging, and that uncontrolled hypertension may cause target organ damage to their remaining kidney, and be counseled on the importance of maintaining a healthy lifestyle and BP control before and after donation.

\section{What Prior Guidelines Recommend}

Multiple prior guidelines for the evaluation and care of living kidney donor candidates identify hypertensive end-organ damage (eg, proteinuria, microalbuminuria, left ventricular hypertrophy, and hypertensive retinopathy), as relative contraindications or exclusion criteria for kidney donation. . $^{38,48,50,54,55,196,214,215}$ Other relative contraindications or exclusion criteria for donation defined in prior guidelines include uncontrolled hypertension ${ }^{48,55,196,214}$; need for more than 1,54 or more than 2 antihypertensive agents $^{214,215}$ for adequate control; hypertension and age younger than 50 years at evaluation ${ }^{196}$; non-white or African American race ${ }^{54,196}$; or the presence of several comorbidities or cardiovascular risk factors. ${ }^{50,196,214}$ We concur that uncontrolled hypertension or hypertension with target organ damage should be exclusions to kidney donation. However, based on our evidence review, we also conclude that there is limited evidence from the donor population to make recommendations for donor acceptance based on hypertension status alone. Rather we endorse adherence to the general framework that compares predicted individual long-term ESKD risk according to baseline profile of demographic and health characteristics including BP to the transplant program's acceptable risk threshold.

\section{RESEARCH RECOMMENDATIONS}

- Define optimal strategies for measuring BP during the donor candidate evaluation.

- Determine optimal strategies for treatment of hypertension in kidney donor candidates before donation, and in accepted donors after donation.

- Quantify the impact of living kidney donation on hypertension risk, as well as the impact of hypertension before and after donation on clinical outcomes including CVD and lifetime ESKD risk.

- Evaluate possible variation in the risk and consequences of hypertension at donation according to other characteristics, including baseline demographic and other clinical factors.

\section{CHAPTER 11: PREDONATION METABOLIC AND LIFESTYLE RISK FACTORS}

The ERT search parameters did not identify evidence from eligible studies pertinent to the recommendations in chapter 11 and therefore the following recommendations are "Not Graded.”

\section{Identification of Metabolic and Lifestyle Risk Factors}

11.1: Risk factors for kidney and cardiovascular disease should be identified before donation and addressed by counseling to promote long-term health.

\section{Obesity}

11.2: Body mass index (BMI) should be computed based on weight and height measured before donation, and classified based on World Health Organization (WHO) criteria for the general population or racespecific categories.

11.3: The decision to approve donor candidates with obesity and $\mathrm{BMI}>30 \mathrm{~kg} / \mathrm{m}^{2}$ should be individualized based on demographic and health profile in relation to the transplant program's acceptable risk threshold.

11.4: Donor candidates who have had bariatric surgery should be assessed for risk of nephrolithiasis.

\section{Glucose Intolerance}

11.5: Donor candidates should be asked about prior diagnosis of diabetes mellitus, gestational diabetes, and family history of diabetes.

11.6: Glycemia should be assessed by fasting blood glucose and/or glycated hemoglobin $\left(\mathrm{HbA}_{1 \mathrm{c}}\right)$ before donation.

11.7: 2-hour glucose tolerance testing or $\mathrm{HbA}_{1 \mathrm{c}}$ testing should be performed in donor candidates with elevated fasting blood glucose, history of gestational diabetes, or family history of diabetes in a first-degree relative, and results should be used to classify diabetes or prediabetes status using established criteria for the general population.

11.8: Donor candidates with type 1 diabetes mellitus should not donate. 
11.9: The decision to approve donor candidates with prediabetes or type 2 diabetes should be individualized based on demographic and health profile in relation to the transplant program's acceptable risk threshold.

11.10: Donor candidates with prediabetes or type 2 diabetes should be counseled that their condition may progress over time and may lead to end-organ complications.

\section{Dyslipidemias}

11.11: Fasting lipid profile (including total cholesterol, LDL-C, HDL-C and triglycerides) should be measured as part of an overall cardiovascular risk assessment before donation.

11.12: The decision to approve donor candidates with dyslipidemia should be individualized based on demographic and health profile in relation to the transplant program's acceptable risk threshold.

\section{Tobacco Use}

11.13: The use of tobacco products should be assessed before donation.

11.14: Donor candidates who use tobacco products should be counseled on the risks of perioperative complications, cancer, cardio-pulmonary disease and kidney failure, should be advised to abstain from use of tobacco products, and should be referred to a tobacco cessation support program if possible.

11.15: The decision to approve donor candidates who are active tobacco users should be individualized based on demographic and health profile in relation to the transplant program's acceptable risk threshold.

\section{RATIONALE}

\section{Goals of Evaluation and Definitions}

This section addresses the evaluation and management of metabolic and lifestyle risk factors associated with ESKD, CVD and/or all-cause mortality, as applicable to the care of donor candidates. Some of the factors considered do not have currently known associations with ESKD risk, but are relevant to a comprehensive predonation health assessment. Even if donation does not increase the risk, traditional CVD risk factors are expected to have at least the same effect in donors as in the general population. Attention to these risk factors is intended to prevent or delay the onset and progression of comorbid diseases, kidney disease, and CVD. All risk factors considered in this section are potentially modifiable by lifestyle and/or medical care.

According to World Health Organization (WHO), the prevalence of diabetes mellitus increased from 108 million people worldwide in 1980 to 422 million in 2014 . $^{246}$ The rising prevalence of diabetes is linked with the obesity epidemic and 2.1 billion adults worldwide were estimated to be overweight or obese in $2013 .^{247}$ Underlying causes of the obesity epidemic may be modifiable, and include sedentary lifestyles, high-fat and energy-dense diets, and increased urbanization. ${ }^{248-250}$ The WHO defines obesity based on thresholds of BMI, a measure of weight scaled for height, as: underweight $\left(<18.5 \mathrm{~kg} / \mathrm{m}^{2}\right)$, normal weight $\left(18.5-24.9 \mathrm{~kg} / \mathrm{m}^{2}\right)$, overweight $\left(25-29.9 \mathrm{~kg} / \mathrm{m}^{2}\right)$, obese $\left(30-34.9 \mathrm{~kg} / \mathrm{m}^{2}\right)$, and morbidly obese $\left(>35 \mathrm{~kg} / \mathrm{m}^{2}\right) \cdot{ }^{248}$ While BMI is recognized to be an imperfect measure of body composition, the components of BMI are easily measured, recorded, and followed over time, and have prognostic implications. Optimal BMI-based thresholds for obesity may differ from WHO standards in non-whites, and race-specific thresholds have been proposed. Measurement of waist circumference and/or waist-to-hip ratios may also be considered to characterize the distribution of adiposity in obese persons. ${ }^{251}$

Multiple prior guidelines and policies for the evaluation and care of living donors recommend measurement of fasting plasma glucose and consideration of oral glucose tolerance testing and/or glycated hemoglobin $\left(\mathrm{HbA}_{1 \mathrm{c}}\right)$ as part of the donor evaluation. ${ }^{38,48,51,196,252}$ The WHO defines diabetes mellitus as: fasting plasma glucose of $7.0 \mathrm{mmol} / \mathrm{L}$ or greater ( $\geq 126 \mathrm{mg} / \mathrm{dL})$, random plasma glucose of $11.1 \mathrm{mmol} / \mathrm{L}$ or greater $(\geq 200 \mathrm{mg} / \mathrm{dL})$, or plasma glucose concentration of $11.1 \mathrm{mmol} / \mathrm{L}$ or greater $(\geq 200 \mathrm{mg} / \mathrm{dL}) 2$ hours after a $75-\mathrm{g}$ anhydrous glucose load in an oral glucose tolerance test. A 2011 addendum included recognition of $\mathrm{HbA}_{1 \mathrm{c}}$ of $48 \mathrm{mmol} / \mathrm{mol}$ or greater $(6.5 \%)$ as an additional criterion for diagnosing diabetes if assays are standardized to criteria aligned with international reference values, but also indicated that $\mathrm{HbA}_{1 \mathrm{c}}$ less than $48 \mathrm{mmol} / \mathrm{mol}$ (6.5\%) does not exclude diabetes diagnosed using glucose tests. ${ }^{246}$

The WHO defines Impaired Glucose Tolerance as fasting plasma glucose less than $7.0 \mathrm{mmol} / \mathrm{L}(<126 \mathrm{mg} / \mathrm{dL})$ or 2-hour post-load plasma glucose of 7.8 or greater and less than $11.1 \mathrm{mmol} / \mathrm{L}$ ( $\geq 140$ and $<200 \mathrm{mg} / \mathrm{dL})$, while Impaired Fasting Glucose is defined as fasting plasma glucose 6.1 to $6.9 \mathrm{mmol} / \mathrm{L}$ (110 to $125 \mathrm{mg} / \mathrm{dL}$ ) or 2-hour post-load plasma glucose less than $7.8 \mathrm{mmol}(<140 \mathrm{mg} / \mathrm{dL}) .{ }^{253}$ Currently there are various definitions of prediabetes and the equivalent term used by WHO, intermediate hyperglycemia, is defined by fasting plasma glucose of 6.1 to $6.9 \mathrm{mmol} / \mathrm{L}(110-125 \mathrm{mg} / \mathrm{dL})$ and 1-hour post-load plasma glucose of 7.8-11.0 $\mathrm{mmol} / \mathrm{L}$ (140-199 mg/dL). ${ }^{254}$

Dyslipidemias are classified based on elevations in total cholesterol and low-density lipoprotein cholesterol (LDL-C), and low concentrations of high-density lipoprotein cholesterol (HDL-C). Hypertriglyceridemia is an independent CVD risk factor. $^{255}$

\section{Obesity}

Increased risk of perioperative complications including wound and surgical site infections in obese patients is well established in the general surgical literature. ${ }^{256}$ The ERT report identified 2 systematic reviews that examined perioperative outcomes after donor nephrectomy according to BMI, with quality of source studies rated as low. Among 8 studies reporting operative time, all but one found a modest increase in operative time (mean difference of 16.9 minutes) among donors with BMI of 30 or greater compared to those with BMI less than $30 \mathrm{~kg} / \mathrm{m}^{2}$ (Supplemental Appendix Table C7, SDC, http://links.lww.com/TP/B432). While warm ischemia times were reported to be longer for obese donors in all but one study, this difference was not significant on meta-analysis. Pooled results showed no differences in blood loss or length of stay among obese compared with normal weight donors.

In the general population, obesity has been identified as a risk factor for diabetes mellitus and it may also be a risk factor for kidney disease, particularly obesity-related glomerulopathy. ${ }^{257}$ The ERT identified 5 studies comparing longterm outcomes among donors by predonation BMI, rated as low quality, with follow-up ranging from 6.7 to 15.1 years (Supplemental Appendix Table D12, SDC, http://links.lww. 
com/TP/B432). One study found that BMI was associated with cardiovascular mortality but not all-cause mortality. ${ }^{32}$ In contrast, another registry study found no association between BMI and death over 12 years. ${ }^{23} \mathrm{~A}$ few studies of variable quality examined associations between BMI and intermediate outcomes. ${ }^{140,244,258}$

Multiple prior guidelines for the evaluation and care of living kidney donor candidates recommend BMI greater than $35 \mathrm{~kg} / \mathrm{m}^{2}$ as an absolute or relative contraindication to donation. $38,48,50,54,196,215,259$ and other guidelines recommend careful evaluation for other comorbidities in donor candidates with BMI greater than $30 \mathrm{~kg} / \mathrm{m}^{2} \cdot{ }^{48,54}$ With regard to other metrics of obesity aside from BMI, the Spanish Society of Nephrology and Spanish National Transplant Organisation guidelines define waistline greater than $82 \mathrm{~cm}$ in women or greater than $102 \mathrm{~cm}$ in men as additional relative contraindications to donation. ${ }^{54}$ CARI guidelines advise measurement of waist circumference within the assessment of overweight and obese donor candidates, ${ }^{259}$ and a US Joint Societies work group consensus statement includes abdominal circumference as part of the definition of metabolic syndrome. ${ }^{196}$ In contrast, the ERT concluded that there is limited evidence from the donor population to make recommendations for donor acceptance based on BMI alone among obese donor candidates.

A recent meta-analysis based on data from nearly 5 million healthy persons identified from 7 general population cohorts found a modest association of BMI greater than $30 \mathrm{~kg} / \mathrm{m}^{2}$ with increased risk of ESKD over median cohort follow-up of 4 to 16 years (adjusted HR, 1.041.161.29). ${ }^{7}$ Projected 15 -year and lifetime risks of ESKD for healthy persons varied according to BMI and age, sex, and race (Figures 16 and 17). A recent study of 119769 US donors published after the ERT
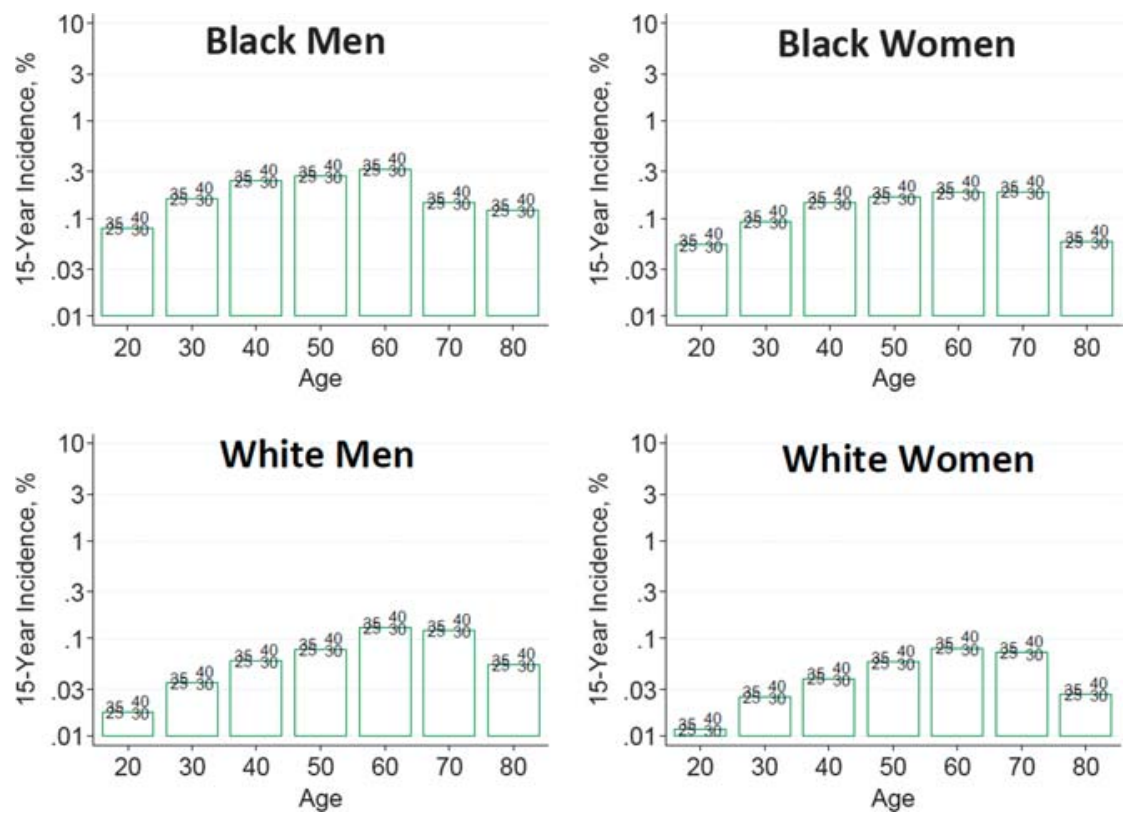

FIGURE 16. Estimated 15-year incidence (\%) of ESKD in the United States according to baseline body mass index (BMI) and demographic profile from the CKD-PC. *The base-case scenario is defined as: age-specific eGFR $\left(114,106,98,90,82,74\right.$, and $66 \mathrm{~mL} / \mathrm{min}^{2}$ per $1.73 \mathrm{~m}{ }^{2}$ for ages 20 , 30, 40, 50, 60, 70, and 80 years, respectively), SBP $120 \mathrm{~mm} \mathrm{Hg}$, urine ACR $4 \mathrm{mg} / \mathrm{g}[0.4 \mathrm{mg} / \mathrm{mmol}]$, BMl $26 \mathrm{~kg} / \mathrm{m}^{2}$, and no diabetes mellitus or antihypertensive medication use. These were selected as being representative of recent US living kidney donors where, with the exception of eGFR, there was little variation in health characteristics by age. ACR, albumin-to-creatinine ratio; CKD-PC, Chronic Kidney Disease-Prognosis Consortium, eGFR, estimated glomerular filtration rate; ESKD, end-stage kidney disease; SBP, systolic blood pressure. Reprinted from Grams ME, Sang Y, Levey AS, et al. Kidney-failure risk projection for the living kidney-donor candidate. N Engl J Med. 2016;374:411-421. ${ }^{7}$ 

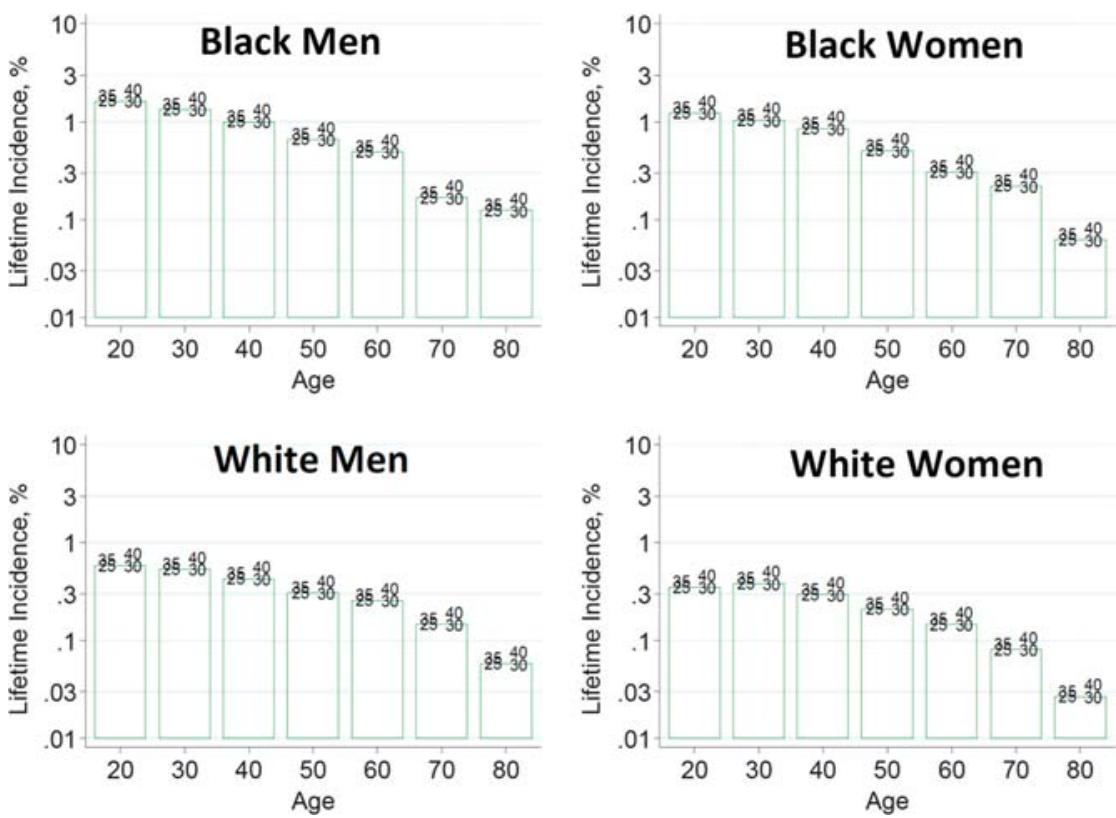

FIGURE 17. Estimated lifetime incidence (\%) of ESKD in the United States according to baseline body mass index (BMI) and demographic profile from the CKD-PC. *The base-case scenario is defined as: age-specific eGFR $\left(114,106,98,90,82,74\right.$, and $66 \mathrm{~mL} / \mathrm{min}^{\mathrm{per}} 1.73 \mathrm{~m}{ }^{2}$ for ages 20 , $30,40,50,60,70$, and 80 years, respectively), SBP $120 \mathrm{~mm} \mathrm{Hg}$, urine ACR $4 \mathrm{mg} / \mathrm{g}[0.4 \mathrm{mg} / \mathrm{mmol}]$, BMl $26 \mathrm{~kg} / \mathrm{m}^{2}$, and no diabetes mellitus or antihypertensive medication use. These were selected as being representative of recent US living kidney donors where, with the exception of eGFR, there was little variation in health characteristics by age. Lifetime risk projections are based on 15 years of follow-up data and calibrated to the incidence of ESKD in the low-risk population, and thus are likely imprecise. ACR, albumin-to-creatinine ratio; CKD-PC, Chronic Kidney Disease-Prognosis Consortium, eGFR, estimated glomerular filtration rate; ESKD, end-stage kidney disease; SBP, systolic blood pressure. Reprinted from Grams ME, Sang Y, Levey AS, et al. Kidney-failure risk projection for the living kidney-donor candidate. N Engl J Med. 2016;374:411-421.

that, compared with nondiabetic persons, those with type 2 diabetes but otherwise good health had 3-times the risk of ESKD over median cohort follow-up of 4 to 16 years
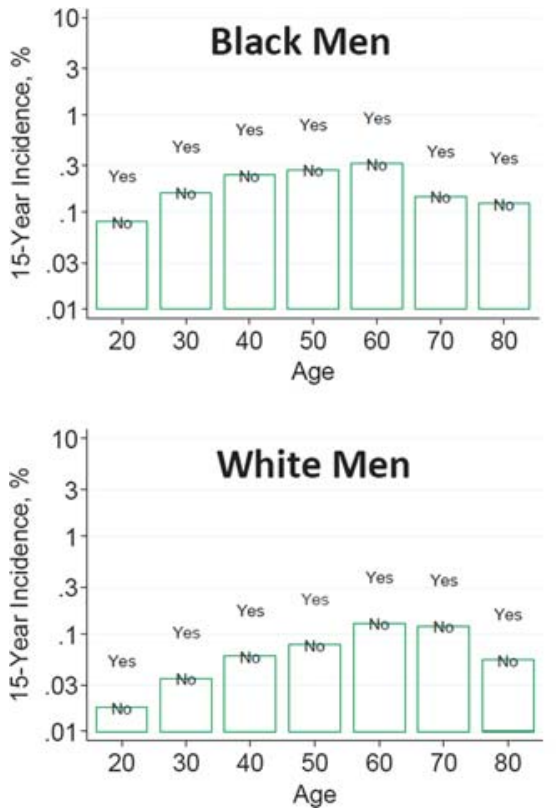

(adjusted HR, $\left.{ }_{1.91} 3.01_{4.74}\right) .^{7}$ The 15 -year and lifetime risks of ESKD for healthy persons varied by type 2 diabetes and age, sex, and race (Figures 18 and 19). Multiple prior
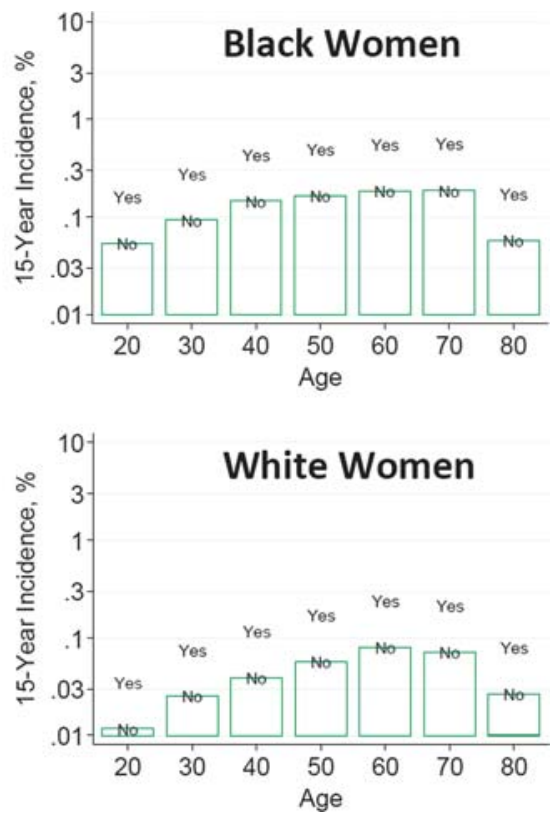

FIGURE 18. Estimated 15-year incidence (\%) of ESKD in the United States according to non-insulin-dependent diabetes mellitus status and demographic profile from the CKD-PC. *The base-case scenario is defined as: age-specific eGFR (114, 106, 98, 90, 82, 74, and 66 mL/min per $1.73 \mathrm{~m}^{2}$ for ages $20,30,40,50,60,70$, and 80 years, respectively), SBP $120 \mathrm{~mm} \mathrm{Hg}$, urine ACR $4 \mathrm{mg} / \mathrm{g}$ [0.4 mg/mmol], BMl $26 \mathrm{~kg} / \mathrm{m}^{2}$, and no diabetes mellitus or antihypertensive medication use. These were selected as being representative of recent US living kidney donors where, with the exception of eGFR, there was little variation in health characteristics by age. ACR, albumin-to-creatinine ratio; BMI, body mass index; CKD-PC, Chronic Kidney Disease-Prognosis Consortium, eGFR, estimated glomerular filtration rate; ESKD, end-stage kidney disease; SBP, systolic blood pressure. Reprinted from Grams ME, Sang Y, Levey AS, et al. Kidney-failure risk projection for the living kidney-donor candidate. N Engl J Med. 2016;374:411-421. ${ }^{7}$ 

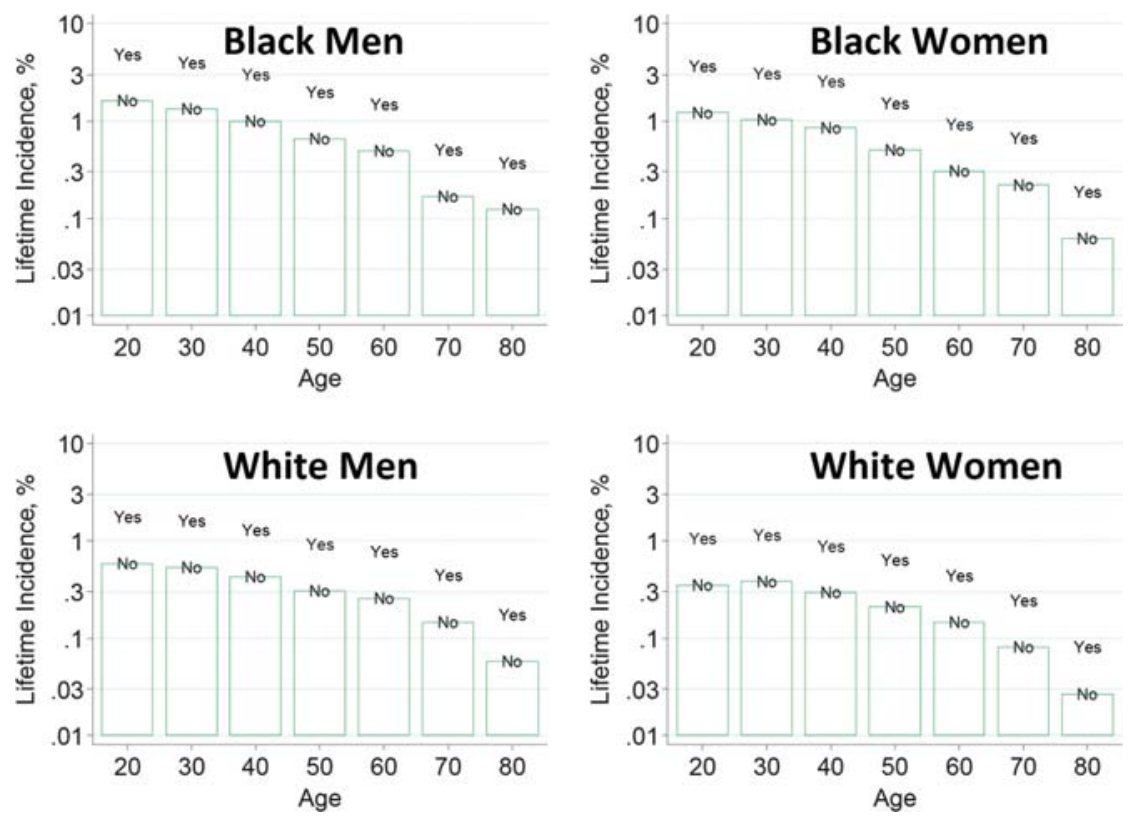

FIGURE 19. Estimated lifetime incidence (\%) of ESKD in the United States according to non-insulin-dependent diabetes mellitus status and demographic profile from the CKD-PC. * The base-case scenario is defined as: age-specific eGFR $(114,106,98,90,82,74$, and $66 \mathrm{~mL} / \mathrm{min}$ per $1.73 \mathrm{~m}^{2}$ for ages $20,30,40,50,60,70$, and 80 years, respectively), SBP $120 \mathrm{~mm} \mathrm{Hg}$, urine ACR $4 \mathrm{mg} / \mathrm{g}\left[0.4 \mathrm{mg} / \mathrm{mmol}^{\prime}, \mathrm{BMl} 26 \mathrm{~kg} / \mathrm{m}^{2}\right.$, and no diabetes mellitus or antihypertensive medication use. These were selected as being representative of recent US living kidney donors where, with the exception of eGFR, there was little variation in health characteristics by age. Lifetime risk projections are based on 15 years of follow-up data and calibrated to the incidence of ESKD in the low-risk population, and thus are likely imprecise. ACR, albumin-to-creatinine ratio; BMl, body mass index; CKD-PC, Chronic Kidney Disease-Prognosis Consortium, eGFR, estimated glomerular filtration rate; ESKD, end-stage kidney disease; SBP, systolic blood pressure. Reprinted from Grams ME, Sang Y, Levey AS, et al. Kidney-failure risk projection for the living kidney-donor candidate. N Engl J Med. 2016;374:411-421. ${ }^{7}$

guidelines for the evaluation and care of kidney donors recommend that diabetic persons be excluded from kidney donation. ${ }^{38,54,179,196,215,252}$ However, the European Best Practice Guideline qualifies an exception of "exceptional circumstances" 215 and the British Transplantation Society recommends that "diabetics can be considered for kidney donation after a thorough assessment of the lifetime risk of cardiovascular and progressive renal disease in the presence of a single kidney." ${ }^{48}$ The WG concluded that persons with type 1 diabetes should be excluded from kidney donation. However, consistent with the framework of integrated risk assessment, we endorse individualizing the decision to approve donation in persons with prediabetes or type 2 diabetes based on the severity of illness and predicted longterm risk (considering complete demographic and health profile) in relation to the transplant program's acceptable risk threshold. The WG considers that older candidates with type 2 diabetes with well-controlled glycemia, not requiring insulin and without end-organ damage, might be considered for donation.

Prediabetes represents an intermediate category of hyperglycemia, which poses increased risks for future type 2 diabetes mellitus and CVD. ${ }^{261}$ Important risk factors for diabetes and prediabetes in the general population include increasing age, high-risk ethnicity or race, obesity, and history of diabetes in a first degree relative. Approximately $5-10 \%$ of individuals with prediabetes progress to diabetes per year ${ }^{264}$ but the risk of progression can be reduced with lifestyle changes and weight loss. ${ }^{265}$ The younger the individual with risk factors for prediabetes, the higher the likelihood that diabetes and subsequent kidney disease will develop in that person's remaining lifetime. ${ }^{266}$
A small cohort study found that carefully screened prediabetic living kidney donors may revert to normal fasting glucose and did not have an increased risk of impaired kidney function in the short term ${ }^{267}$ (Evidence Report Table 17, SDC, http:// links.lww.com/TP/B434; Supplemental Appendix Table D14, SDC, http://links.lww.com/TP/B432). However, quality of this study was very low. A recent study linking US national donor registry data to ESKD registry data for 125427 living donors examined causes of ESKD after donation. ${ }^{31}$ The finding that early postdonation ESKD was predominantly reported as due to glomerulonephritis while late postdonation ESKD was more frequently reported as diabetic-ESKD and hypertensive-ESKD could be consistent with an effect of donation on diabetes and hypertension as causes for ESKD. These findings emphasize the importance of counseling donor candidates with prediabetes regarding their increased lifetime risk for progression to diabetes and subsequent end-organ complications, and the importance of healthy lifestyle behaviors to reduce risks. Those who are approved to donate should be counseled on the importance of regular medical follow-up after donation including surveillance for hyperglycemia and kidney function for many decades after donation, and prompt institution of appropriate management.

Prior recommendations regarding candidacy of persons with prediabetes for kidney donation are conflicting. The European Best Practice Guideline states that impaired glucose tolerance is not an absolute contraindication to donation ${ }^{50,215}$; other guidelines consider prediabetes a relative contraindication ${ }^{54,196}$ or a condition warranting careful consideration, ${ }^{48}$ while CARI considers prediabetes as well as past history of gestational diabetes to be absolute contraindications. ${ }^{252}$ Given the lack of current data specific to the donor population, we endorse individualizing the decision to approve donation in persons with 
prediabetes based on their predicted long-term risk in relation to the transplant program's acceptable risk threshold.

\section{Dyslipidemias}

Dyslipidemia is a modifiable risk factor for CVD, and hypertriglyceridemia and low HDL-C are components of the metabolic syndrome. With regard to associations of lipid levels with outcomes after donation, one study identified by the ERT compared kidney function and albuminuria at 5 years among donors with versus without predonation metabolic syndrome (Evidence Report Table 18, SDC, http://links. lww.com/TP/B434; Supplemental Appendix Table D15, SDC, http://links.lww.com/TP/B432). Although small differences in the outcomes were suggested, statistical comparisons were not performed. No studies were identified comparing postdonation outcomes based on lipid status alone. A recent meta-analysis based on data from nearly 5 million persons identified from 7 general population cohorts found no associations of total cholesterol or LDL-C with ESKD risk over median cohort follow-up of 4 to 16 years. $^{7}$

Several prior guidelines recommend fasting lipid profiling as part of the donor evaluation, ${ }^{51,54,59,196}$ but do not define donor selection criteria based on lipid levels alone. A US Joint Societies work group consensus statement recognizes hypertriglyceridemia and low HDL-C as components of the metabolic syndrome, and considers impaired fasting glucose and other components of metabolic syndrome to be relative contraindications to donation in persons younger than age 50 years. ${ }^{196} \mathrm{We}$ endorse individualizing the decision to approve donation in persons with dyslipidemia based on their predicted long-term risk (considering complete demographic and health profile) in relation to the transplant program's acceptance threshold.

\section{Tobacco Use}

Cigarette smoking is a strong, modifiable risk factor for CVD (as well as other adverse health outcomes such as lung disease and cancer); smoking may also cause direct renal damage through microvascular injury and promotion of atherosclerosis, and has been associated with CKD in the general population. ${ }^{268,269}$ A recent meta-analysis based on data from nearly 5 million persons identified from 7 general population cohorts found that, compared with nonsmokers over median cohort follow-up of 4 to 16 years, current smokers had a $76 \%$ increase in the risk of ESKD (adjusted HR, $1.291 .76_{2.41}$ ) and past smokers had a $45 \%$ increase in risk (adjusted HR, $\left.{ }_{1.23} 1.45_{1.71}\right){ }^{7}$ After calibration to annual ESKD incidence in the US healthy population, variations in the projected 15-year and lifetime risks of ESKD based on smoking status were generated according to age, sex, and race for healthy persons (assuming age-specific GFR, urine ACR $4 \mathrm{mg} / \mathrm{g}$ [0.4 mg/mmol], SBP $120 \mathrm{mmHg}$, BMI $26 \mathrm{~kg} / \mathrm{m}^{2}$, and absence of diabetes mellitus; Figures 20 and 21).

Although data on the outcomes associated with smoking among living kidney donors are limited, an analysis of linked US transplant registry and national death records found that while smoking was not significantly associated with perinephrectomy mortality, donors who smoked had approximately 5 -times the adjusted mortality over 12 years compared with nonsmoking donors. ${ }^{23}$ See chapter 4 (Preoperative Evaluation) for a discussion of the smoking-related risks before general surgery.

Some prior guidelines for the evaluation and care of living donors advise assessment of smoking, but make no recommendations concerning smoking in the selection of donors. ${ }^{51,196}$ Others advise encouraging smoking cessation
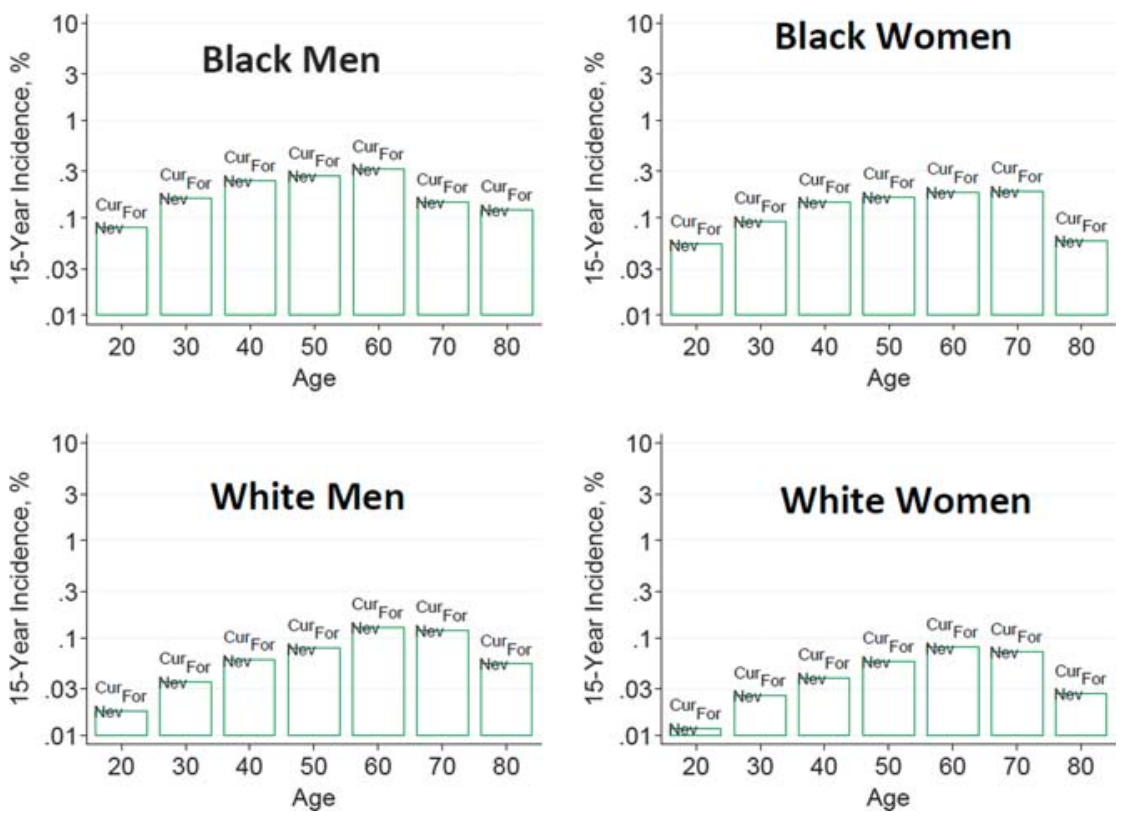

FIGURE 20. Estimated 15-year incidence (\%) of ESKD in the United States according to baseline smoking status and demographic profile from the CKD-PC. *The base-case scenario is defined as: age-specific eGFR $\left(114,106,98,90,82,74\right.$, and $66 \mathrm{~mL} / \mathrm{min}$ per $1.73 \mathrm{~m}{ }^{2}$ for ages 20, 30, 40, 50, 60, 70, and 80 years, respectively), SBP 120 mm Hg, urine ACR 4 mg/g [0.4 mg/mmol], BMl, 26 kg/m²; and no diabetes mellitus or antihypertensive medication use. These were selected as being representative of recent US living kidney donors where, with the exception of eGFR, there was little variation in health characteristics by age. Smoking status, current (Cur), former (For), and Never (Nev). ACR, albumin-to-creatinine ratio; BMl, body mass index; CKD-PC, Chronic Kidney Disease-Prognosis Consortium, eGFR, estimated glomerular filtration rate; ESKD, end-stage kidney disease; SBP, systolic blood pressure. Reprinted from Grams ME, Sang Y, Levey AS, et al. Kidney-failure risk projection for the living kidney-donor candidate. N Engl J Med. 2016;374:411-421. ${ }^{7}$ 

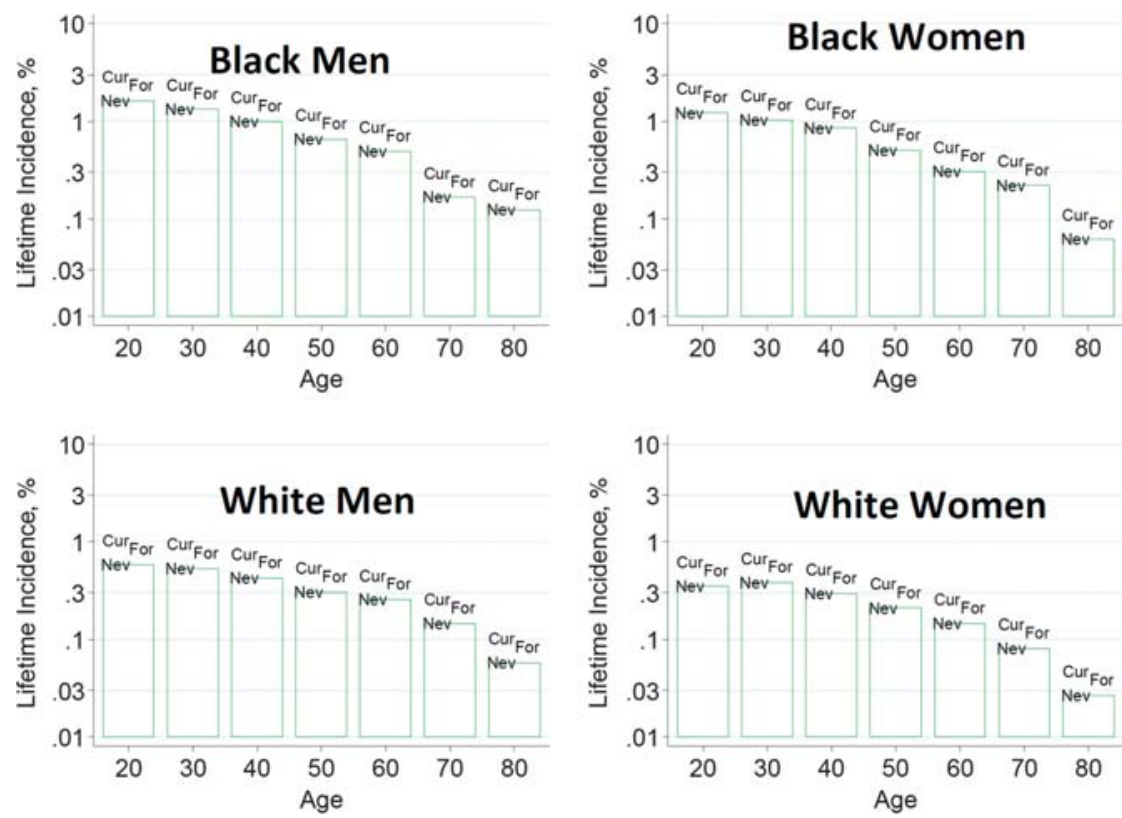

FIGURE 21. Estimated lifetime incidence (\%) of ESKD in the United States according to baseline smoking status and demographic profile from the CKD-PC. *The base-case scenario is defined as: age-specific eGFR $\left(114,106,98,90,82,74\right.$, and $66 \mathrm{~mL} / \mathrm{min}^{\mathrm{p}}$ per $1.73 \mathrm{~m}{ }^{2}$ for ages 20,30 , 40, 50, 60, 70, and 80 years, respectively), SBP $120 \mathrm{~mm} \mathrm{Hg}$, urine ACR $4 \mathrm{mg} / \mathrm{g}$ [0.4 mg/mmol], BMl $26 \mathrm{~kg} / \mathrm{m}^{2}$, and no diabetes mellitus or antihypertensive medication use. These were selected as being representative of recent US living kidney donors where, with the exception of eGFR, there was little variation in health characteristics by age. Lifetime risk projections are based on 15 years of follow-up data and calibrated to the incidence of ESKD in the low-risk population, and thus are likely imprecise. Smoking status, current (Cur), former (For), and Never (Nev). ACR, albumin-to-creatinine ratio; BMl, body mass index; CKD-PC, Chronic Kidney Disease-Prognosis Consortium, eGFR, estimated glomerular filtration rate; ESKD, end-stage kidney disease; SBP, systolic blood pressure. Reprinted from Grams ME, Sang Y, Levey AS, et al. Kidneyfailure risk projection for the living kidney-donor candidate. N Engl J Med. 2016;374:411-421.7

without defining an exclusion criterion. ${ }^{48,50,215}$ Two guidelines recommend smoking cessation 4 weeks before donor nephrectomy, ${ }^{38,54}$ and guidelines from the Spanish Society of Nephrology and Spanish National Transplant Organisation emphasize long-term abstinence. ${ }^{54}$ The 2000 National Kidney Foundation (NKF)/AST guidelines recommend considering acceptance of donor candidates who smoke only if they abstain form use of tobacco products for 6 months and have normal pulmonary studies. ${ }^{18}$ We endorse individualizing the decision to approve donation in active smokers based on their predicted long-term risk (considering complete demographic and health profile) in relation to the transplant program's acceptance threshold.

\section{Impact of Donation on Cardiac Risk}

The ERT identified 2 studies comparing cardiovascular events in donors versus healthy nondonors (very low quality of evidence; Evidence Report Table 8, SDC, http://links.lww. com/TP/B434; Supplemental Appendix Table D5, SDC, http://links.lww.com/TP/B432). In a study comparing 2028 living kidney donors in Ontario, Canada (1992 to 2009) with 20280 healthy, demographically matched nondonors, the risk of death or major cardiovascular events over a median 7 years follow-up (maximum, 18 years) was lower in donors than in healthy nondonors (2.8 vs 4.1 events per 1000 person-years, adjusted HR, $\left.0.480 .66_{0.90}\right) .{ }^{270}$ The risk of death-censored major cardiovascular events was similar among donors and nondonors (1.7 vs 2.0 events per 1000 person-years).

A study with longer follow-up compared cardiovascular and all-cause mortality in 1901 kidney donors with a group of 32621 healthy, matched controls selected from a population-based survey in Norway (Nord-Trøndelag Health
Study [HUNT] I). ${ }^{32}$ Mortality was similar in donors versus nondonors over the first 15 years, but at 25 years after donation, cumulative all-cause mortality was approximately $18 \%$ among donors versus $13 \%$ among healthy nondonors (adjusted HR ${ }_{1.1} 1.3_{1.5}$ ). Limitations of this study include differences in accrual periods and in baseline characteristics (including age) between the donors and nondonors. ${ }^{271,272}$ Continued study is needed to assess the impact of donation on long-term survival in large, representative cohorts.

Limited data are available on the impact of donation on the pathophysiology of CVD. The Chronic Renal Impairment in Birmingham (CRIB)-Donor study included 68 donors at 2 United Kingdom centers (2011 to 2014), of whom $90 \%$ were white, and prospectively examined changes in left ventricular mass and other CVD surrogate markers at 12 months postdonation versus predonation, compared with changes in these parameters in healthy nondonors. ${ }^{202}$ Donors had a greater increase in left ventricular mass $(+7 \pm 10$ vs $-3 \pm 8 \mathrm{~g} ; P<0.001)$ and mass:volume ratio $(+0.06 \pm 0.12$ vs $-0.01 \pm 0.09 \mathrm{~g} / \mathrm{mL} ; P<0.01)$, but decreased aortic distensiblity; donors were also more likely than controls to develop detectable highly sensitive troponin $\mathrm{T}$ levels. The increase in left ventricular mass among donors was independently associated with the magnitude of decrease in mGFR. These pilot observations warrant replication efforts in larger cohorts with longer follow-up to better define the impacts of donation on CVD surrogates and clinical events.

\section{RESEARCH RECOMMENDATIONS}

- Evaluate the outcomes of donors with metabolic and lifestyle risk factors for CVD, including perinephrectomy complications, 
long-term mortality, CVD events, CKD/proteinuria and ESKD. Develop integrated risk assessment tools tailored for demographic and health profiles.

- Quantify the direct effects of donation on CVD risk.

- Assess effectiveness of predonation interventions including counseling and weight or lifestyle changes on long-term donor outcomes.

- Assess the impact of the duration and durability of predonation weight loss on donor acceptance and postdonation outcomes in those who proceed to donation.

\section{CHAPTER 12: PREVENTING INFECTION TRANSMISSION}

The ERT search parameters did not identify evidence from eligible studies pertinent to the recommendations in chapter 12 and therefore the following recommendations are "Not Graded.”

\section{Evaluation}

12.1: Risk for human immunodeficiency virus (HIV), hepatitis $\mathrm{B}$ virus $(\mathrm{HBV})$, and hepatitis $\mathrm{C}$ virus $(\mathrm{HCV})$ infections should be assessed before donation.

12.2: Donor candidates should be assessed for factors associated with an increased likelihood of endemic or unexpected infections, including geographic, seasonal, occupational, animal and environmental exposures.

12.3: Donor candidates should complete a urinalysis and testing for HIV, HBV, HCV, cytomegalovirus (CMV), Epstein-Barr virus (EBV), and Treponema pallidum (syphilis).

12.4: If indicated by regional epidemiology or individual history, donor candidates should complete testing for Mycobacterium tuberculosis, Strongyloides, Trypanosoma cruzi, West Nile virus, Histoplasmosis, and/or Coccidiomycosis.

12.5: Transplant programs should develop protocols to screen donor candidates for emerging infections in consultation with local public health specialists.

12.6: In general, donor infection risk factor and microbiological assessments should be performed or updated as close in time to donation as possible. For HIV, $\mathrm{HBV}$ and HCV, screening should be current within 28 days of donation.

\section{Selection}

12.7: If a donor candidate is found to have a potentially transmissible infection, then the donor candidate, intended recipient and transplant program team should weigh the risks and benefits of proceeding with donation.

\section{RATIONALE}

\section{Goals of Evaluation and Definitions}

The goals of infection screening in donor candidates are to identify illnesses that may affect the donor candidate or the intended recipient. ${ }^{273-276}$ Evaluation of donor candidates to reduce the risk of transmissible infections should include assessment of the individual's history of past infections and infectious disease risk factors (eg, risk of local endemic infections or travel to endemic areas), awareness of current patterns of geographically endemic infections, and focused microbiological screening.

Donor-derived infections can be classified as "expected" versus "unexpected." 274,276 The risks of "expected" donor-derived infection transmission are defined by donor and recipient screening, such as in high-risk scenarios for transmission of cytomegalovirus (CMV), Epstein-Barr virus (EBV) or toxoplasmosis from a seropositive donor to a seronegative recipient. "Expected" transmissions occur frequently and are managed by surveillance and/or prophylaxis strategies in the recipient after transplantation. 277-282 "Unexpected" donor-derived infections arise despite routine donor screening, such as human immunodeficiency virus (HIV) or hepatitis $\mathrm{C}$ virus (HCV) transmission due to false negative serologic testing or infection in a "window period." The 2013 US Public Health Service (PHS) Guideline provides an evidence-based instrument to screen for behavioral factors associated with recent $\mathrm{HIV}$, hepatitis $\mathrm{B}$ virus (HBV), or HCV infection (Table 16). ${ }^{71}$

TABLE 16.

US Public Health Service (PHS) 2013 Screening for factors associated with increased likelihood of recent HIV, HBV, or HCV infection

- Donors who meet one or more of the following criteria should be identified as being at increased risk for recent HIV, HBV, and HCV infections.

- Each factor listed reflects increased risk of all 3 pathogens as an aggregate, as there is overlap of associated risk, even though each factor does not convey risk from all pathogens equally.

- The first 6 factors address sexual contact; the definition of "had sex" refers to any method of sexual contact, including vaginal, anal and oral contact.

1. Have you had sex with a person known or suspected to have HIV, HBV, or HCV infections in the preceding 12 mo?

2. If male: Have you had sex with another man in the preceding 12 mo?

3. If female: Have you had sex with a man with a history of male-sex-with-male behavior in the preceding 12 mo?

4. Have you had sex in exchange for money or drugs in the preceding $12 \mathrm{mo}$ ?

5. Have you had sex with a person that has injected drugs (by intravenous, intramuscular, or subcutaneous route) for nonmedical reasons in the preceding 12 mo?

6. Have you injected drugs (by intravenous, intramuscular, or subcutaneous route) for nonmedical reasons in the preceding 12 mo?

7. Have you been in lockup, jail, prison, or a juvenile correctional facility for more than $72 \mathrm{~h}$ in the preceding $12 \mathrm{mo}$ ?

8. Have you been newly diagnosed with or have been treated for syphilis, gonorrhea, Chlamydia, or genital ulcers in the preceding 12 mo?

- Donors who meet the following criterion should be identified as being at increased risk for recent HCV infection only:

9. Have you been on hemodialysis in the preceding $12 \mathrm{mo}$ ?

US PHS risk factors also include: 10. A child who is $\leq 18$ months of age and born to a mother known to be infected with, or at increased risk for HIV, HBV, or HCV infection. 11. A child who has been breastfed within the preceding 12 months and the mother is known to be infected with, or at increased risk for, HIV infection. HBV, hepatitis B virus; HCV, hepatitis C virus; HIV, human immunodeficiency virus; PHS, Public Health Service. Adapted with permission from Seem DL, Lee I, Umscheid CA, et al. Excerpt from PHS guideline for reducing HIV, HBV and HCV transmission through organ transplantation. Am J Transplant. 2013;13:1953-1962. ${ }^{71}$ 
"Unexpected" infectious disease transmissions through organ transplantation are rare, but may result in serious morbidity and mortality. ${ }^{274,283}$ Although most "unexpected" disease transmissions have involved deceased donors, cases of HIV and HCV transmissions from living donors have been reported. ${ }^{283,284}$ Notably, while reporting of suspected or documented donor-derived infection transmissions is required in the United States, reporting is voluntary in many other countries, and thus true incidence may be underestimated.

Infection transmission events may also be categorized according to the certainty that the donor is the origin of the infection, as opposed to reactivation or de novo infection in the recipient. ${ }^{285}$ Consensus-based definitions have been offered to standardize categorization as: "proven," "probable," "possible," "unlikely," "excluded," "intervened upon without documented transmission," and "positive assay without apparent disease transmission" events. 285 "Proven" denotes clear evidence of the same infectious disease in the donor and at least one of the recipients, while "probable" is based on strong evidence suggesting but not proving a disease transmission. Use of standardized nomenclature may facilitate global tracking and study of such infectious disease transmissions as well as the comparison of data between published studies and reports collected globally. ${ }^{285}$

The risk of donor-derived disease transmission can be mitigated by the donor evaluation, including history taking (clinical, social/behavioral, travel) and microbiological testing. While microbiological testing should be performed in all donors for some pathogens [HIV, HBV, HCV, CMV, EBV, syphilis], focusing testing for other pathogens based on regional epidemiology and individual clinical, social or geographic risk factors should reduce the likelihood of procuring an organ that could transmit infection, while preserving opportunities for donation (avoiding false-positive test results) (Table 17). Approaches to screening should consider the virulence of a particular pathogen, available testing assays, and residual window periods for transmission despite screening ${ }^{283,286}$ as discussed according to specific pathogen below. ${ }^{71,273,275,276,286-291}$ Risks versus benefit must be balanced in the decision to use organs from infected donors, incorporating predonation treatment and recipient prophylaxis where appropriate. It is also necessary to inform the recipient and their care team of any known risks from the potential donated kidney.

\section{Hepatitis B Virus}

Evaluation of donor candidates should include US PHS risk factor screening for increased risk of HBV infection. ${ }^{71}$ All donor candidates should undergo testing for IgG hepatitis $\mathrm{B}$ core antibody (anti-HBcAb) and hepatitis B surface antigen (HBsAg). ${ }^{71,273,275}$ HBV DNA nucleic acid testing (NAT) can further stratify transmission risk in donor candidates from $\mathrm{HBV}$ endemic areas who are anti-HBcAb+, those with possible mutant $\mathrm{HBV}$ infections, and those with abnormal liver function tests or a past history of liver disease of unknown etiology. Testing for HBV should be performed as close as possible to the date of the organ recovery, but within no longer than 28 days before donation. ${ }^{71}$

Transplantation of kidneys from HBsAg+ donors is contraindicated for $\mathrm{HBV}$ - recipients, but may be considered for $\mathrm{HBsAg}+$ recipients or recipients with $\mathrm{HBV}$ protective immunity, ${ }^{275}$ with informed consent of the recipient, possible antiviral HBV treatment of the recipient and posttransplant monitoring. Kidney transplant recipients from anti-HBcAb +/HBsAg-/HBV DNA- donors appear to have little risk of acquiring active HBV infection. ${ }^{275,292}$ HBV NAT testing should be performed in donor candidates with isolated $\mathrm{HBcAb}+$ status to further define risk of transmission. If the donor is anti-HBc + and HBV DNA-, the risk of transmission is negligible, ${ }^{293}$ especially if the recipient is anti-HBsAb + or has been effectively immunized against HBV. Still, the recipient should be informed of the small potential risk of disease transmission, and posttransplant monitoring should be performed.

\section{Hepatitis C Virus}

Evaluation of donor candidates should include US PHS risk factor screening for increased risk of HCV infection. ${ }^{71}$ Additional HCV risk factors identified by the US Centers for Disease Control for the general public (not specific to organ donation) include: persistently abnormal alanine aminotransferase concentrations, receipt of blood transfusion or blood components before 1992, receipt of clotting factor concentrates produced before 1987, recognized exposure among healthcare workers, and children born from $\mathrm{HCV}+$ mothers. ${ }^{294}$ Regardless of past risk factors, all donor candidates should undergo testing for HCV infection as close as possible to the date of the organ recovery, but within no longer than 28 days before donation. ${ }^{71}$ Thus, behavioral risk factor assessment is used to inform pretest probability for interpretation of microbiological test results and to guide counseling to avoid infection after testing, not to determine which donor candidates should be tested. Approximately $15 \%$ of people with anti-HCV antibodies will not have detectable HCV-RNA in the serum. The 2013 US PHS guideline recommends that all living donor candidates should be tested for both anti-HCV antibody and for HCV RNA by NAT. ${ }^{71}$

Before the advent of new direct-acting antiviral agents, active $\mathrm{HCV}$ infection in a donor candidate was considered a contraindication to living donation, not only because of the risk of transmitting $\mathrm{HCV}$ to the recipient but also because of the risk of glomerular disease in the donor. ${ }^{295} \mathrm{HCV}$ has been transmitted to naïve organ recipients from infected living and deceased donors. ${ }^{296}$ Organ transplantation from an $\mathrm{HCV}+$ donor is associated with significant risk of $\mathrm{HCV}$ transmission, especially to $\mathrm{HCV}$ - recipients. ${ }^{297}$ However, how to handle the situation when an otherwise suitable living kidney donor is $\mathrm{HCV}+$ may evolve in the era of effective treatment with direct-acting antiviral agents. It is of course best to use a living donor who is not HCV+. However, if research protocols are developed to assess living kidney donation from $\mathrm{HCV}+$ persons, then regardless of the HCV status of the recipient it would make sense that protocols require donor treatment with direct-antiviral agents for at least 12 weeks, the treatment duration when most studies show sustained virologic response, before donating a kidney. ${ }^{298}$

\section{Human Immunodeficiency Virus}

Evaluation of donor candidates should include US PHS risk factor screening for increased risk of HIV infection. ${ }^{71}$ All donor candidates should undergo microbiological testing for HIV infection as close as possible to the date of the organ recovery, but within no longer than 28 days before donation. ${ }^{71,275} \mathrm{HIV}$ infection is a contraindication to organ donation to HIV- recipients as the transmission of HIV by organ 


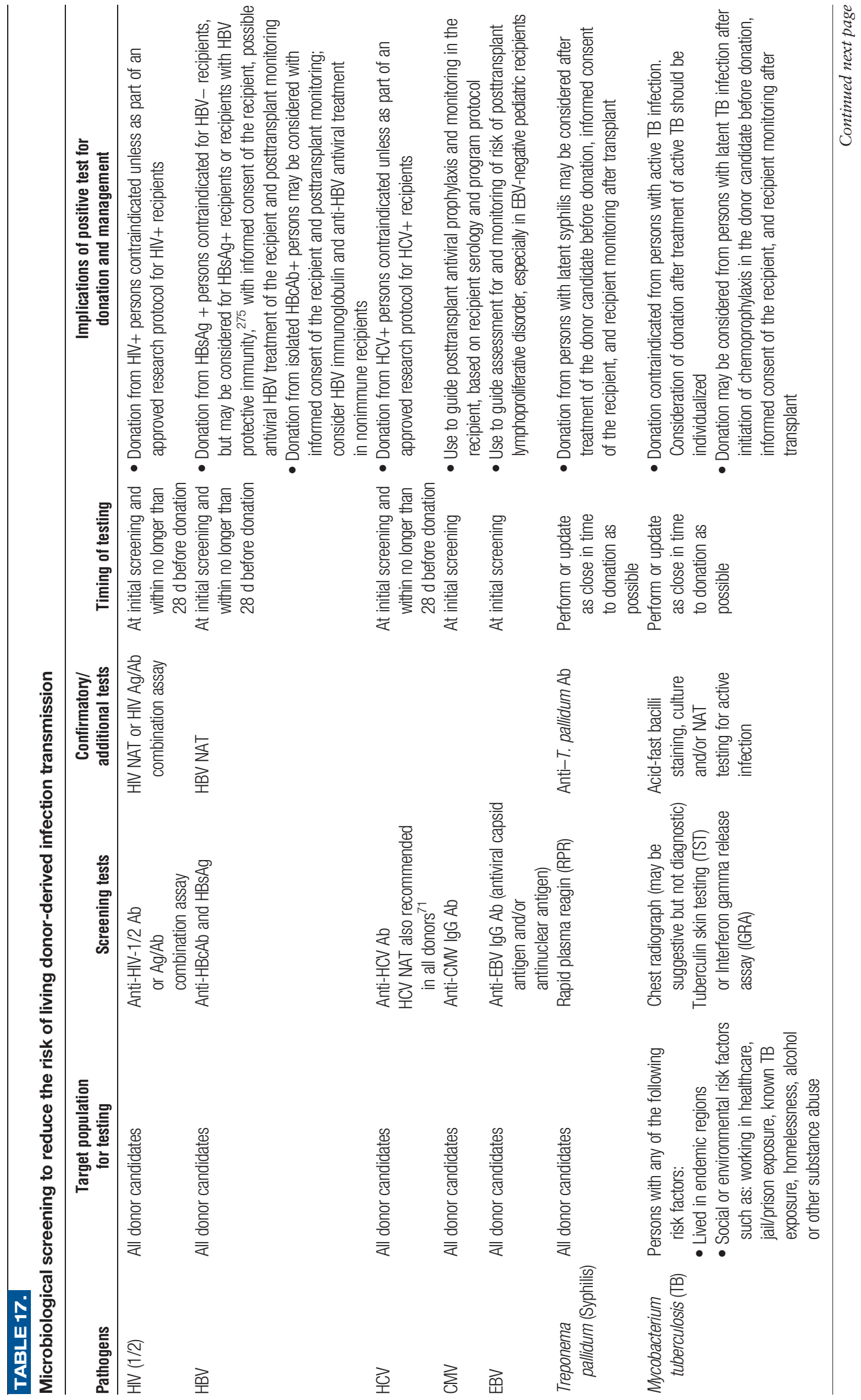




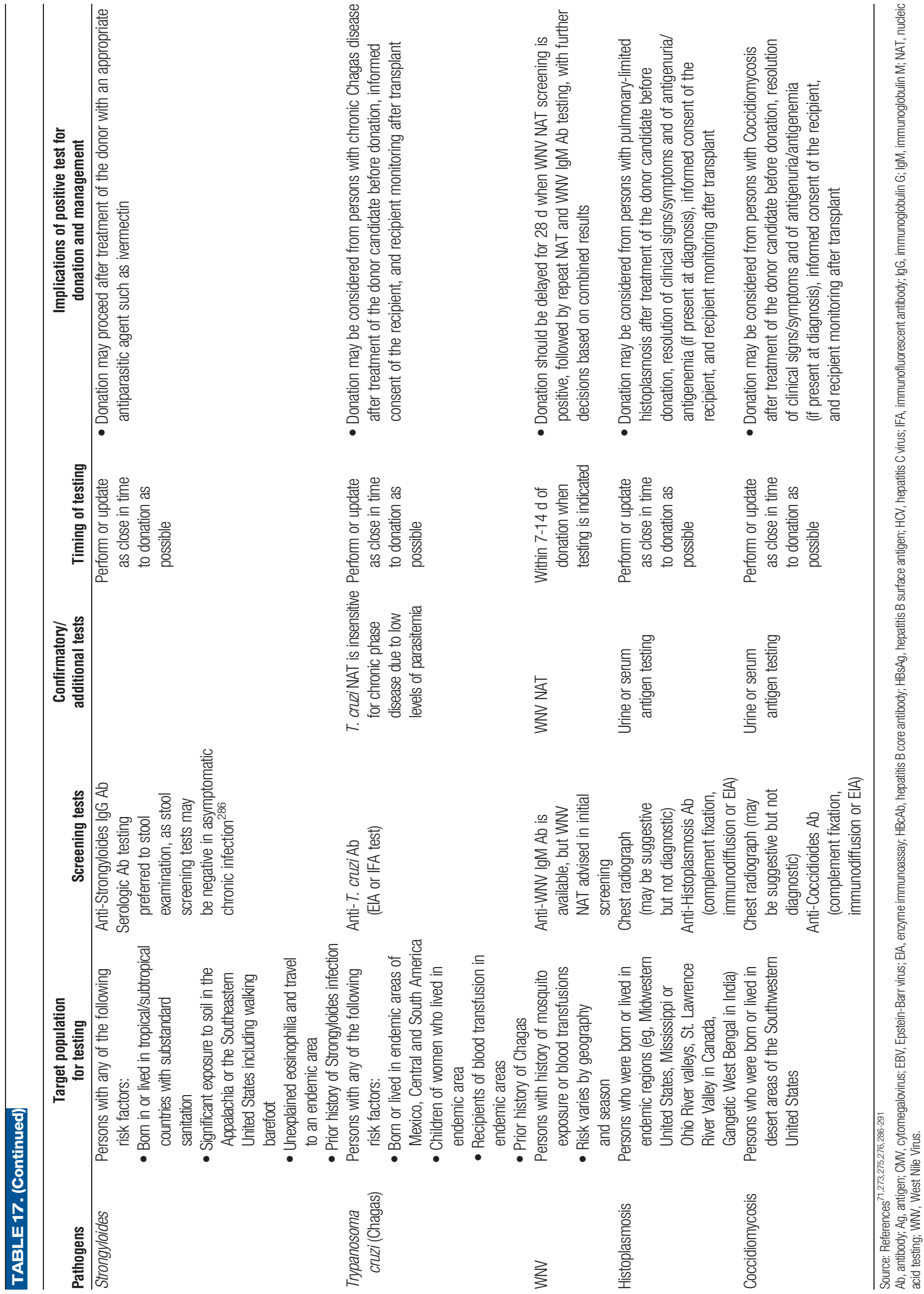


transplantation is well documented. Tests to detect HIV include antibodies generated against HIV antigens, direct detection of viral nucleic acid (NAT testing) or HIV antigen p24. Currently, antibodies against HIV antigens remain the most commonly used method for detection of HIV. The period from HIV exposure to the development of HIV antibodies is approximately 22 days, but can be up to 6 months. Thus the donor may be seronegative while potentially infectious. ${ }^{283}$ NAT testing can reduce the window period for HIV to between 5.6 and 10.2 days. ${ }^{299}$

In contrast to undetected donor disease transmission, medical advancements in HIV antiviral therapy have led to consideration of planned kidney transplantation from HIV+ donors to HIV+ recipients, such as recent experience described in South Africa. ${ }^{300}$ In the United States, the National Organ Transplant Act (NOTA) of 1984 prohibited the knowing procurement or transplantation of organs from an HIV infected donor, but in 2013 the HIV Organ Policy Equity (HOPE) Act repealed this prohibition and authorized the OPTN to develop standards for use of organs from known HIV infected individuals in HIV infected recipients. At this time, such donations and transplantation should occur only within the context of research protocols; protocols in the United States were developed by the National Institutes of Health (NIH). ${ }^{301}$

\section{Increased Risk Donors and Window Periods for HBV, HCV and HIV}

Serological testing for infections has been highly effective in reducing the risks of donor-derived disease transmission. However, seroconversion requires the elaboration of antibodies against a specific pathogen and could be delayed for several weeks after infectious exposure. Testing during the window period for seroconversion may generate falsenegative test results and could lead to inadvertent infection transmissions. Cases of donor-derived infection transmissions related to window period infections missed by serologic screening of donors have been reported. ${ }^{274,283}$ The period from HIV exposure to the development of HIV antibodies is 22 days on average, but can be up to 6 months. ${ }^{274} \mathrm{HBsAg}$ enzyme-linked immunosorbent assays (ELISAs) have a window period of 38.3 to 49.7 days, while the time from HBV exposure to positive NAT testing ranges from 20.4 to 25.7 days. The window period for detection of HCV infection by ELISA is 38 to 94 days, but the duration of the window is substantially reduced to 6.1 to 8.7 days by the use of NAT. ${ }^{274}$

In 2007 in the United States, a previously uninfected deceased donor kidney transplant recipient tested positive for HIV and HCV infection. Routine donor serologic screening for HIV and HCV infection was negative; the donor's only known risk factor for HIV was having sex with another man. Four organs (2 kidneys, liver and heart) were transplanted to 4 recipients. NAT of donor sera and posttransplant sera from all recipients were positive for HIV and HCV. ${ }^{302}$ This case highlighted the potential for donors to harbor HIV and HCV infection during the window period, when infection cannot be detected by antibody screening.

In 2009, a case of unexpected HIV transmission from a living organ donor in New York City was also reported. ${ }^{284}$ Based on this case, it was suggested that to reduce the risk for transmission of HIV through living donor organ transplantation, transplant programs should screen living donors for HIV as close to the time of organ recovery as possible, using sensitive tests for both chronic and acute infections, namely, antibody and NAT testing. ${ }^{284}$

In 2013, the US PHS updated their "Guideline for Reducing HIV, HBV and HCV Transmission through Organ Transplantation," including recommended risk factor assessment in all donor candidates (Table 16). ${ }^{71}$ Living donor candidates with behaviors associated with increased risk of acquiring HIV, HBV or HCV identified during evaluation should receive individualized counseling on specific strategies to prevent exposure to these viruses during the period before donation surgery. Recommendations regarding microbiological testing include:

- All potential organ donors (living or deceased) should be tested for antibodies to HIV (ie, anti-HIV $1 / 2 \mathrm{Ab}$ or HIV antigen/antibody $[\mathrm{Ag} / \mathrm{Ab}]$ combination assay). All potential organ donors identified as being at increased risk for HIV infection should also be tested for HIV RNA by NAT or HIV antigen (eg, HIV Ag/Ab combination assay). Donor blood specimens should be obtained before procurement. $\mathrm{Ab}$ or $\mathrm{Ag} / \mathrm{Ab}$ test results should be made available before transplantation.

- All potential organ donors (living or deceased) should be tested for both anti-HCV Ab and for HCV RNA by NAT. Donor blood specimens should be obtained before procurement. Antibody test results should be made available before transplantation.

- All potential organ donors (living or deceased) should be tested for anti-HBcAb and for HBsAg. Donor blood specimens should be obtained before procurement. $\mathrm{Ag} / \mathrm{Ab}$ test results should be made available before transplantation.

- As noted above, the guideline recommends that all living donor candidates should be tested for HIV, HBV and HCV as close as possible to the date of the organ recovery operation, but within no longer than 28 days before surgery.

Whether retesting closer to the time of transplantation (eg, within 7 to 10 days before donation) is warranted to detect new infections and reduce the window period, overall or among high-risk donor candidates, remains controversial. ${ }^{303,304} \mathrm{~A}$ survey of living donor transplant programs in New York State in 2012 found that most responding programs had policies to retest living donors within 14 days of donation and while there were rare cases of delays in donation associated with repeat testing, no cancellations occurred. ${ }^{305}$

\section{Epstein Barr Virus}

The presence of anti-EBV antibodies signifies prior donor infection, with potential for reactivation of the latent virus and subsequent infection of the immunosuppressed recipient. While detection of the EBV in the living donor generally will not preclude donation, knowing that the kidney comes with latent EBV infection may be important in posttransplant recipient care. ${ }^{306}$ Infection with EBV manifests as a spectrum of diseases ranging from asymptomatic viremia to infectious mononucleosis to posttransplant lymphoproliferative disorder (PTLD). ${ }^{307}$ EBV disease and associated PTLD are more frequently seen when primary EBV infection occurs after transplant, a common scenario in EBV- pediatric solid organ transplant recipients who receive a kidney from an $\mathrm{EBV}+$ donor. In the United States, the cumulative 1- and 5-year incidence of PTLD in 2010 was reported to be $1.3 \%$ and $2.4 \%$, respectively, for pediatric kidney recipients but less 
than $0.2 \%$ and $0.6 \%$ respectively, for adult recipients. ${ }^{307}$ When the donor is EBV+ and the recipient is EBV-, particularly in pediatric recipients, clinical vigilance is required after transplantation to detect PTLD. ${ }^{308}$ Intensity of EBV viral load and immunosuppressive therapies influence the risk for PTLD. ${ }^{307}$

\section{Cytomegalovirus}

Cytomegalovirus disease may result from reactivation of latent infection or primary infection transmitted by a kidney from a CMV+ donor. The laboratory methods for CMV diagnosis are serology, culture, antigenemia, and molecular methods such as CMV NAT, which is most commonly performed using real-time polymerase chain reaction. ${ }^{309}$ The main clinical utility of CMV serology is stratification of a transplant recipient's risk of CMV disease based on donor and recipient status. $279,280,310$

The presence of anti-CMV antibodies in a donor candidate indicates prior infection, with the potential that the latent virus will reactivate and cause infection, particularly in the CMV- recipient. The detection of anti-CMV antibodies does not preclude donation, and infection risk can be anticipated and managed. Primary CMV infection is generally more severe than reactivation and recipients at highest risk are those who are CMV seronegative and receive a kidney transplant from a CMV seropositive donor. Matching CMV seronegative recipients with CMV seronegative donors is an effective strategy for reducing the risk of CMV infection but is rarely practical in the context of living donor kidney transplantation. CMV seropositive recipients may develop disease reactivation or donor-related infection. Thus organ donors and recipients should be tested for prior (latent) CMV infection using anti-CMV antibody for risk stratification and guidance of appropriate surveillance and/or antiviral prophylaxis after transplantation. ${ }^{311}$

\section{Syphilis}

Transmission of syphilis by organ transplantation has been documented. ${ }^{312}$ In the United States, all assays currently FDA-approved for detecting evidence of T. pallidum infection in organ and tissue donors are serologic assays. ${ }^{313}$ There are 2 types of serologic assays: nontreponemal and treponemal. Nontreponemal assays use a combination of cardiolipin, cholesterol, and other lipid substances released from damaged cells as the antigenic source to detect antibodies against cardiolipin, which circulates in the sera of individuals infected with syphilis and may also be present in individuals with a variety of other conditions. Reactivity to cardiolipin generally disappears within a year or 2 after successful treatment of syphilis. ${ }^{313}$ Treponemal assays detect T. pallidum antibodies, which tend to remain elevated for life. Therefore, treponemal assays cannot distinguish between recent, remote, and previously treated infection. Donors are screened for serological evidence of syphilis with a nontreponemal assay such as the rapid plasma reagin (RPR) or venereal disease research laboratory (VDRL) test, which should be confirmed later with a treponemal $\mathrm{Ab}$ immunoassay. A recent study found that current screening of deceased organ donors by RPR yields a significant number of false-positive results. Use of alternative tests or the routine use of confirmatory tests may reduce the frequency of false-positive syphilis results in potential deceased and living organ donors. $^{314}$
As there are multiple available syphilis assays providing different types of information, no single blood assay can conclusively define an individual's disease status. For donor candidate testing, specimen collection and the time available to perform testing must be considered in choosing an appropriate donor screening assay. ${ }^{313}$ Living donor candidate evaluation is less time constrained than the screening of deceased donors, and thus screening with nontreponemal assays followed by confirmation with treponemal assays is preferred if feasible.

Transmission of syphilis has been reported in the United Kingdom to 2 recipients from a deceased donor with a past history of treated disease, supporting recommendations of penicillin for treatment of recipients of deceased donor organs from serologically reactive donors. ${ }^{312}$ Donation from living persons with latent syphilis may be considered after treatment of the donor candidate before donation (eg, with penicillin), informed consent of the recipient, and recipient monitoring after transplant.

\section{Tuberculosis (TB)}

The incidence of posttransplant TB varies substantially depending on the local prevalence of TB infection, which ranges from $1 \%$ in Germany to nearly $14 \%$ in India. ${ }^{290}$ Studies in the United States and Europe have estimated that $0.35 \%$ to $6.6 \%$ of transplant recipients develop TB (across organ and donor types), and that $4 \%$ posttransplant TB cases are donor-derived. ${ }^{290,315} \mathrm{~TB}$ is one of the more common bacterial causes of donor-derived infection in the United States. ${ }^{316}$

Consensus-based recommendations for the diagnosis and management of TB in organ donors include: $288,290,317$

- Risk stratification of all donor candidates, according to:

- Place of birth, residence or travel to a geographically endemic region, with increased risk defined by residence greater than 3 months or relief work in a high prevalence region

- Social risk factors including working in healthcare, prison exposure/incarceration, known TB contact, homelessness, alcohol or other substance abuse

- Medical risk factors including history of untreated TB or radiographic evidence of prior TB; underweight BMI and diabetes have also been correlated with increased TB risk

- Chest radiograph in all donor candidates

- Consideration of urinalysis with microscopy, genitourinary imaging, urine acid-fast bacilli smear and culture in living donor candidates from countries with intermediate to high TB prevalence

- Consideration of immune-based diagnostic testing by tuberculin skin testing (TST) or the interferon-gamma release assay (IGRA)

Diagnostic tests for latent TB infection are limited in sensitivity and have a relatively low predictive value for development of active TB. ${ }^{318}$ The specificity of TST is related to the burden of TB in that region or country, and IGRA has superior specificity in populations where use of Bacillus Calmette-Guérin vaccination is common based on use of specific antigens absent in Bacillus Calmette-Guérin strains Immune testing of all donor candidates or selective testing based on risk profile are considered acceptable options

- Persons with positive immune-based TB testing who are asymptomatic and do not have signs of active TB are considered to have latent TB infection

- Donation from persons with active TB is contraindicated. Risk of transmission from donors with prior appropriately 
treated active TB appears to decline with longer time from treatment. Donation may be considered with informed consent of the recipient, consideration of recipient chemoprophylaxis under the guidance of an infectious disease specialist, and recipient monitoring after transplant.

- Living donor candidates with latent TB infection should be offered chemoprophylaxis according to local or national guidelines. Donation may be considered from persons with latent TB infection with informed consent of the recipient and recipient monitoring after transplant. As there are no data on optimal duration of treatment before donation, individualization of the timing of donation in relation to start of donor chemoprophylaxis is recommended. Chemoprophylaxis of recipients from donors with latent $\mathrm{TB}$ infection should also be considered, especially if the donor did not complete chemoprophylaxis before donation. ${ }^{317}$

A recent study in Korea, a country with an intermediate prevalence of TB, prospectively evaluated living donors using the TST and Mycobacterium tuberculosis-specific enzymelinked immunosorbent spot (ELISPOT) IGRA. ${ }^{319}$ Among the 205 living donors, $31 \%$ had a positive TST and $47 \%$ had a positive ELISPOT. Based on the high rate of suspected latent TB infection detected by screening living donors using TST and ELISPOT in a country with a TB intermediate prevalence, the authors recommended further study of the cost effectiveness of recipient TB chemoprophylaxis. ${ }^{319}$

\section{Urine Culture}

Urine should be sent for culture from all donor candidates at evaluation, and ideally repeated close to the time of donation (eg, within the preceding 2 weeks). Acute symptomatic urinary tract infection is a reason to postpone donation. However, detection of asymptomatic bacteriuria is not infrequent, especially in female donors. A history of urinary tract infection in a donor candidate, particularly if there is a family history of reflux nephropathy, or in a male, requires detailed imaging of the kidneys (eg, assessment for cortical scarring). Any active bacterial or fungal infection in the donor should be treated and, ideally, resolved before donation and transplantation. ${ }^{283}$ Antibiotic prophylaxis should be given to the recipient if resolution of infection is not confirmed before donation, as a positive urine culture early after transplantation, even when asymptomatic, may be associated with increased risk of acute rejection in the recipient. ${ }^{320,321}$

\section{Seasonal and Geographically Endemic Infections}

The donor candidate evaluation should include assessment of place of residence, travel, seasonal, occupational, and recreational risks, as well as prior infections in the donor candidate and family members (Table 17). A number of geographically endemic and seasonal diseases have been transmitted through organ donors including: Strongyloides, Trypanosoma cruzi (Chagas disease), West Nile virus, histoplasmosis, coccidiomycosis, Aspergillus, toxoplasmosis, malaria, Creutzfeldt-Jacob disease, human T-cell lymphotrophic virus infection, and schistosomiasis. $^{273,275,286}$ Other viral, fungal, bacterial and parasitic pathogens recognized as sources of organ donorderived infection transmissions are listed in Table 18. Living donation affords sufficient time for microbiological testing, donor treatment and deferral of donation and transplantation until resolution of infection. Organ donation may be possible after treatment of the donor candidate before donation,
TABLE 18.

Social and clinical factors associated with increased likelihood of geographically endemic infections and infections related to specific exposures

Geographic risks (including duration of time spent in a location)

- Home country/region

- Place of birth (outside vs inside home region)

- Prolonged residence outside home region (recent or distant)

- Occupational or recreational travel to other countries and/or regions

- Countries of origin for close family members

- Ingestion of well water

Occupational risks

- Healthcare workers

- Veterinarians, animal care workers

- Landscapers, park rangers, and other outdoor workers

- Occupations with international travel, such as Peace Corps, international journalists

- Medical mission trips (consider a 3-mo washout period before donation to allow identification of subclinical disease)

\section{Seasonal risks}

- Warm weather and insect exposure — eg, local West Nile Virus, dengue, Chikungunya virus, local rickettsial infections, Lyme disease Hobbies

- Hunting/dressing game, taxidermy

- Time living outdoors including camping, swimming in lakes, drinking stream water, insect exposures

- Adventure sports

- Gardening

Significant animal exposure (wild and/or domestic)

- Large numbers of cats or dogs or any unusual pets, including whether pets reside mainly indoors or outdoors

- Laboratory/research animals

- Veterinarian/veterinarian assistant

Family members and close contacts with potential risk factors

- Geographic or seasonal infections previously diagnosed in close family members or other contacts may predict risk for subclinical infection in donor candidate

Personal history of seasonal or geographic infection in donor candidate, even if remote

Adapted from OPTN Ad Hoc Disease Transmission Advisory Committee ${ }^{287}$

informed consent of the recipient, as well as recipient monitoring and possible prophylaxis after transplant (Table 19).

Strongyloidiasis typically occurs only in the setting of specific environmental exposures. Donor-derived Strongyloides hyperinfection cases with high associated mortality have been reported, including from kidney transplantation. ${ }^{322-324}$ Consensus-based recommendations of the 2013 AST Infectious Diseases Community of Practice work group and the OPTN Ad Hoc Disease Transmission Advisory Committee (DTAC) support screening for Strongyloides in the following potential organ donors ${ }^{286,287}$ :

- Persons who were born in or lived in tropical or subtropical countries where sanitation conditions are substandard, including candidates with prior military service in endemic areas. The WHO emphasizes a correlation between improved sanitation and human waste disposal with the disappearance of Strongyloidiasis. ${ }^{325}$ Strongyloidiasis has occurred in most countries with the exception of Canada, Japan and Northern Europe. 
TABLE 19.

Recognized organ donor-derived infection transmissions

\begin{tabular}{ll}
\hline Viruses & Bacteria \\
Adenovrius & Mycobacterium tuberculosis \\
BK polyoma virus & Nocardia spp. \\
Cytomegalovius & Rickettsia rickettsii (Rocky \\
Epstein-Barr virus & Mountain Spotted Fever) \\
Herpes simplex virus & Treponema pallidum (syphillis) \\
HIV & Borrelia (Lyme disease) \\
HBV & \\
HCV & \\
Hepatitis E virus & \\
Human T-cell lymphotropic & \\
virus 1 and 2 & \\
Influenza A/B & \\
Lymphocytic choriomeningitis virus & \\
Parvovirus B19 & \\
Rabies & \\
West Nile virus & \\
Fungi & \\
Aspergillus spp. & \\
Candida spp. & \\
Coccidioides immitis & Parasites \\
Cryptococcus neoformans & Babesia microti \\
Histoplasma capsulatum & Balamuthia mandrillaris \\
Scopulariopsis brevicaulis & Malaria spp. \\
Zygomycetes (Mucor) & Naegleria fowleri \\
& Toxoplasma gondii \\
\hline
\end{tabular}

$\mathrm{HBV}$, hepatitis $\mathrm{B}$ virus; $\mathrm{HCV}$, hepatitis $\mathrm{C}$ virus; $\mathrm{HV}$, human immunodeficiency virus. Adapted by permission from Macmillan Publishers Ltd: Fishman JA, Grossi PA. Donor-derived infection —-the challenge for transplant safety. Nat Rev Nephrol. 2014;10:663-672. ${ }^{274}$

- Persons with significant exposure to soil in Appalachia or the Southeastern United States, including walking barefoot.

- Persons with unexplained eosinophilia and travel to endemic area.

- Persons reporting a prior history of Strongyloides infection.

Serologic Ab testing is the preferred screening test for Strongyloides infection, as the sensitivity of stool testing is limited and multiple stool screening tests may be negative in asymptomatic chronic infection. ${ }^{286}$ Strongyloides IgG antibody testing (ELISA-based) is available in many reference labs.

Infected donor candidates should be treated with a minimum of 2 doses of ivermectin before donation $(200 \mu \mathrm{g} / \mathrm{kg}$ orally daily on 2 consecutive days) ${ }^{286,287}$ Because of the potential for persistence of migrating larvae and eggs in the tissues, some experts recommend repeating this treatment 2 weeks later to cover an autoinfection cycle. After treatment, followup laboratory testing of the donor candidate before donation to confirm cure has been deemed unnecessary, unless reexposure has occurred ${ }^{286,287}$

Chagas disease is transmitted through contact with infected triatomine "kissing" bugs, and residents of poorly constructed housing where these insects reside are at greatest risk of acquiring infection. Transmission has also been reported from mother to infant, through blood transfusion, and through organ transplantation. Consensus-based recommendations of the 2011 Chagas in Transplant Working Group, the 2013 AST Infectious Diseases Community of Practice work group, and OPTN DTAC support screening in the following potential organ donors ${ }^{286,287,289}$ :
- Those who were born in or lived in an endemic region in Mexico, Central or South America

- Children of women who lived in endemic regions and whose T. cruzi infection status is positive or unknown

- Persons who received a blood transfusion in endemic regions

- Persons reporting a prior history of Chagas disease

Assessment of outcomes of 32 transplant recipients who received organs from 14 T. cruzi seropositive donors in the United States from 2001 to 2011 confirmed transmission in 9 recipients from 6 donors, including 2 of $15(13 \%)$ kidney recipients, 2 of $10(20 \%)$ liver recipients and 3 of $4(75 \%)$ heart recipients. ${ }^{326}$ Recommended monitoring posttransplant comprised regular testing by polymerase chain reaction, hemoculture, and serologic testing. Thirteen recipients had no or incomplete monitoring; transmission was confirmed in 5 of these recipients; 4 of the 5 recipients had symptomatic disease and all 4 died, although death was directly related to Chagas disease in only one. Nineteen recipients had partial or complete monitoring for T. cruzi infection with weekly testing by polymerase chain reaction, hemoculture and serologic testing; transmission was confirmed in 4 of the 19 recipients with no cases of symptomatic disease. Based on such evidence, recent guidelines support consideration of kidney donation from donors with T. cruzi infection on an individual basis with consent of the recipient. ${ }^{286,287,289}$ Recipients must be informed of the need for participation in close monitoring and the available therapeutic interventions in the event of infection, as the medications available for treatment are not FDA-approved and are generally only available through specific protocols. Consideration of the recipient's access to testing and monitoring is imperative, as geographic concerns may impact the ability to follow the recipient closely.

West Nile Virus is a flavivirus that is transmitted by mosquitoes in an enzootic cycle with birds. When testing is indicated, screening living donor candidates by West Nile virus NAT within 7 to 14 days of donation has been recommended. ${ }^{273,291}$ Proposed strategies for when to begin testing living donor candidates for West Nile virus include when regional blood banks start performing NAT screening or testing during a defined period of time that reflects the peak of local West Nile virus infection. ${ }^{291}$ The 2013 AST Infectious Diseases Community of Practice work group recommended delaying donation for 28 days when NAT screening is positive, followed by repeat NAT and immunoglobulin M (IgM) Ab testing with the following management pathways based on the results ${ }^{273}$ :

- NAT+: Defer donation for at least 120 days. Donation deemed likely to be safe if clearance of viremia demonstrated by NAT testing after 120 days

- NAT-/IgM Ab-: Consider initial NAT testing to reflect a false positive result. Donation may be considered after infectious disease consult

- NAT-/IgM Ab+: Suggests infection with clearance of viremia. Donation may be considered after infectious disease consult

\section{Emerging Infections}

Transplant programs must maintain awareness of new and emerging infections that may be transmissible through organ donation. Availability of microbiological testing for new infections may be limited or available only at specialized laboratories, emphasizing the importance of careful assessment of exposure history in the donor candidate evaluation. 
For example, during the Ebola virus outbreak of 2014, the OPTN DTAC recommended that evaluation of candidates for organ donation should include screening for travel and epidemiologic risk factors for Ebola exposure, including ${ }^{327}$ :

- Travel to a country where an Ebola outbreak occurred within the past 21 days

- Contact with blood, other body fluids, or human remains of a patient known or suspected to have Ebola

- Exposure as a healthcare worker to patients known to have Ebola

- Direct handling of bats or nonhuman primates from diseaseendemic areas

If risk factors for Ebola are identified, the OPTN DTAC recommends aborting the evaluation process and excluding donation.

Zika virus is a mosquito-borne pathogen that gained attention in association with an outbreak of primary microcephaly among children born to infected mothers in Brazil in 2015, followed by rapid geographic spread across the Americas, prompting recognition as a global health emergency. ${ }^{328}$ Other complications of Zika virus infection include acute autoimmune neuropathies such as Guillain-Barré syndrome. Guidance from the OPTN DTAC, the AST, and the ASTS recommend considering donor deferral if there is history of travel to Zika-endemic areas in the 28 days before donation. ${ }^{329}$ In the case of potential living donors with Zika infection, donation should be deferred where possible.

Transplant programs should develop and maintain screening protocols to address emerging infections, including awareness of evolving geographic exposure patterns in consultation with local public health specialists. Current information on global health outbreaks are reported by organizations such as the US Centers for Disease Control. ${ }^{330}$

\section{What Prior Guidelines Recommend}

US OPTN Policy for Medical Evaluation Requirements for Living Donors mandates similar screening tests as recommended in our current guideline: anti-CMV Ab, anti-EBV $\mathrm{Ab}$, anti-HIV 1,2 Ag/Ab, HBsAg, anti-HBcAb, anti-HCV $\mathrm{Ab}$, and syphilis testing. ${ }^{51}$ US transplant programs are required to determine whether the donor has TB exposure risk factors and to test accordingly. US programs are also required to develop protocols to determine who to screen for geographically endemic and seasonal infections such as Strongyloides, Trypanosoma cruzi and West Nile virus.

The British Transplantation Society has also recommended testing for HBV, HCV, EBV, CMV and HIV as part of donor candidate evaluation. ${ }^{48}$ The Spanish Society of Nephrology and Spanish National Transplant Organization recommendations for living donor kidney transplantation included the following as routine tests in the donor candidate evaluation: HIV [a], Hepatitis B: HBsAg [a], anti-HBcAb IgM/IgG [b], HBsAb, HBV DNA in plasma if anti-HbcAb+, Hepatitis C (ELISA and polymerase chain reaction) [a], CMV IgG/IgM [b], EBV IgG/IgM [b], Toxoplasma test, Syphilis (RPR- fluorescent treponemal antibody) [b], Brucella $[\mathrm{b}]$. Here, [a] stands for 'Donation is contraindicated with positive results' and [b] stands for 'Donors and/or recipients have to undergo treatment with positive results' ${ }^{54}$

In contrast to this recommendation, we believe that testing for Toxoplasma and Brucella should be guided by geography and risk factors for possible exposure. Also, since the Spanish Society of Nephrology and Spanish National Transplant Organization guidelines were published in $2010,{ }^{54}$ new research published above supports revision of some categories of [a] 'Donation is contraindicated with positive results.'

\section{RESEARCH RECOMMENDATIONS}

- Define the incidence of donor-derived disease transmission through improved monitoring and reporting. This is critical since determining the relative importance of specific pathogens and risk mitigation strategies requires collection of global data.

- Develop and validate risk assessment questionnaires and protocols for living donor-derived infections, taking into consideration behavioral, occupational, hobby-related, geographic and seasonal exposures.

- Optimize and standardize methods of microbiological assays for donor infection screening and diagnosis.

- Assess results of planned US NIH studies of transplantation from HIV+ donors to HIV+ recipients to develop guidance on appropriate consideration of such transplants in clinical practice.

- Determine, through clinical trials or observational protocols with informed consent, whether kidney donation and transplantation can be performed with acceptable safety and outcomes for the donor and recipient in the following scenarios:

- Donation from $\mathrm{HCV}+$ living donors to $\mathrm{HCV}+$ recipients after antiviral treatment of the donor

Donation from HBsAg + living donors to $\mathrm{HBsAg}+$ recipients or recipients with $\mathrm{HBV}$

\section{CHAPTER 13: CANCER SCREENING}

The ERT search parameters did not identify evidence from eligible studies pertinent to the recommendations in chapter 13 and therefore the following recommendations are "Not Graded.”

\section{Evaluation}

13.1: Donor candidates should undergo cancer screening consistent with clinical practice guidelines for the country or region where the donor candidate resides. Transplant programs should ensure that screening is current according to guideline criteria at the time of donation.

\section{Selection}

13.2: In general, donor candidates with active malignancy should be excluded from donation. In some cases of active malignancy with low transmission risk, a clear management plan and minimal risk to the donor, donation may be considered.

13.3: A kidney with a small simple (Bosniak I) cyst can be left in the donor, particularly if there are compelling reasons for donating the contralateral kidney.

13.4: Donation of a kidney with a Bosniak II renal cyst should proceed only after assessment for the presence of solid components, septations, and calcifications on the preoperative computed tomography scan (or magnetic resonance imaging) to avoid accidental transplantation of a kidney with cystic renal cell carcinoma.

13.5: Donor candidates with high grade Bosniak renal cysts (III or higher) or small (T1a) renal cell carcinoma curable by nephrectomy may be acceptable for donation on a case-by-case basis. 
13.6: Donor candidates with a history of treated cancer that has a low risk of transmission or recurrence may be acceptable for donation on a case-by-case basis.

\section{RATIONALE}

\section{Goals of Evaluation}

The goals of malignancy screening are two-fold. First, it is necessary to identify cancers to protect the health of the donor candidate. Reduced kidney function may compromise long-term health outcomes in individuals requiring cancer treatments with nephrotoxic or cardiovascular side effects (eg, some chemotherapies or radiation treatments). Potential psychosocial stresses of living donation may also be prohibitive in individuals faced with stress of an active cancer diagnosis and treatment. Second, the evaluation must mitigate risks of donor-derived malignancy transmission to the transplant recipient.

\section{General Population Cancer Screening and Incidence}

Most jurisdictions have regional recommendations for which members of the general population should be screened for common cancers, including frequency of screening and acceptable testing modalities. These include screening recommendations for colon, breast, cervical, prostate, and lung cancer. ${ }^{331-333}$ There are potential harms associated with cancer screening, as with any form of screening, if additional testing and procedures are undertaken in patients who ultimately do not have cancer. These risks should be included in the consent for evaluation of the living donor candidate. Transplant programs in countries without local clinical practices guidelines can refer to guidelines from countries or regions most similar to their population. ${ }^{331-333}$

The limited available data on cancer diagnoses after living kidney donation support that donor evaluation and selection practices reduce the incidence of postdonation cancer below that of general population controls, although risk reduction may dissipate with time after donation. ${ }^{334}$ However, cases of cancer diagnoses including melanoma and uterine cancer within less than 1 year of donation have been reported, ${ }^{334}$ emphasizing the need for up-to-date assessment for malignancy before donation.

\section{Recurrence Risk after Treated Cancer}

Recurrence rates after treated cancer from the general population may be used to guide observation periods after cancer treatment before considering organ donation. Average times to recurrence vary by cancer type. Consideration of living donation from a person with a history of treated cancer should include consultation with the donor candidate's oncologist to confirm that individual case factors are associated with "low" (eg, less than 1\%) risks of both lifetime recurrence and disease transmission, and that long-term surveillance will not require frequent imaging that may be restricted by reduced GFR (eg, CT scans with iodinated contrast or magnetic resonance imaging (MRI) scans requiring gadolinium).

\section{Donor-Derived Malignancy Transmission}

Cases of malignancy transmission from deceased or living organ donors to recipients have been reported. A recent systematic review examined all case reports, case series and registry studies describing the outcomes of kidney transplant recipients with donor-derived cancer transmission published through December 2012. ${ }^{335}$ Among 104 donor-transmitted cancer cases identified from 69 studies, the most common transmitted cancer types were renal cancer $(n=20,19 \%)$, followed by melanoma $(\mathrm{n}=18,17 \%)$, lymphoma $(\mathrm{n}=15$, $14 \%)$ and lung cancer $(n=9,9 \%)$. Recipients with transmitted renal cancers had the best outcomes, with more than $70 \%$ of recipients surviving for at least 24 months after transplantation. Patients with melanoma and lung cancers had the worst prognosis, with less than $50 \%$ of recipients surviving beyond 24 months from transplantation. While these data support that donor-derived cancer transmission is uncommon, potential reporting-bias prevents accurate incidence estimates. This report highlights the high mortality associated with donor-derived melanoma and lung cancer transmission.

A history of melanoma is particularly concerning when evaluating a living donor candidate. Aside from the potential for late recurrence and subsequent complications in the donor, melanoma transmission to transplant recipients has been reported after apparent dormancy in the donor for decades, supporting the ability of melanoma cells to remain dormant at distant sites for decades and then reactivate upon exposure to immunosuppression, ${ }^{336,337}$ and transmission can be fatal. The Israel Penn International Transplant Tumor Registry, a voluntary registry of more than 250 cases of organs transplanted from donors with a history of malignancy that captures tumor histology, donor risk factors, method of tumor presentation and recipient outcome, described 13 donors with a history of melanoma (but deemed free of the disease at donation) who provided organs to 28 recipients. ${ }^{338,339}$ Melanoma transmission occurred in 21 recipients (75\%), of whom 13 (62\%) died from metastatic disease. The time to diagnosis ranged from 2.5 to 42 months (median, 10.5 months), and the only patients who survived were those who underwent nephrectomy and cessation of immunosuppression. While some prior general population guidelines such as the US Preventative Services Task Force state that there is insufficient evidence to recommend routine whole body skin exam screening among general adults, ${ }^{340}$ skin examinations for donor candidates with increased recreational or occupational exposure to sunlight, family or personal history of skin cancer, or clinical evidence of precursor lesions may be warranted. Pathology reports of living donor candidates with a prior history of skin cancer resection should be reviewed to ensure that the cancer was not a melanoma before approving donation.

\section{Selection}

In 2011, the OPTN DTAC Malignancy Subcommittee published a classification of 6 risk categories for donorderived malignancy transmission and suitability of organ donation from persons with active or prior malignancy histories ${ }^{341}$; this was also recently reviewed in Kirchner et al. ${ }^{273}$ Classification was based on review of cancer registry reports, published literature, and data submitted to the OPTN. This article did not differentiate between cancer transmissions from living compared with deceased donors due to limited data.

- "No significant risk" was defined as benign tumors where malignancy has been excluded. 
- "Minimal risk" was defined as tumors with $0 \%$ to $0.1 \%$ transmission events per organ transplanted from donors with the specific tumor, and includes nonmelanoma skin cancers, noninvasive carcinoma of the bladder (for nonrenal transplants only), small papillary or follicular carcinoma of the thyroid and solitary, well-differentiated $(\leq 1 \mathrm{~cm})$ renal cell carcinoma.

- "Low risk" (0.1-1\% transmission events per organ transplanted from affected donors) includes small renal cell carcinoma $(1-2.5 \mathrm{~cm})$, low grade central nervous system (CNS) tumors, primary CNS mature teratoma, solitary papillary thyroid carcinoma $(0.5-2.0 \mathrm{~cm})$, minimally invasive follicular carcinoma $(1.0-2.0 \mathrm{~cm})$, and history of treated non-CNS malignancy ( $\geq 5$ years prior) with greater than $99 \%$ probability of cure.

- "Intermediate risk" (1-10\% transmission events per organ transplanted from affected donors) includes breast and colon carcinoma in situ, resected well differentiated renal cell carcinoma $(4-7 \mathrm{~cm})$ and history of treated non-CNS malignancy ( $\geq 5$ years prior) with probability of cure between $90-99 \%$.

- "High risk" (>10\% transmission events per organ transplanted from affected donors) includes current or past history of melanoma, leukemia/lymphoma or neuroendocrine tumors, breast or colon cancer stage 1 or higher, choriocarcinoma, any CNS tumor with vetriculoperitoneal or ventriculoarterial shunt, metastasis or high grade (III/IV) histology, metastatic carcinoma, sarcoma, lung cancer Stage I-IV, and renal cell carcinoma greater than $7 \mathrm{~cm}$. The high risk category also included any treated non-CNS malignancy with insufficient follow-up to predict behavior, incurable or with $<90 \%$ probability of cure, or any other active cancer not previously classified.

- Tumors of "unknown risk" were defined as a final category.

The authors suggested that donors in the "no significant risk" category are standard, and that organs from donors with "minimal risk" malignancies may be used for transplantation based on clinical judgment with informed consent of the recipient. The authors also proposed that organs from donors with "intermediate risk" malignancies could be considered for transplantation with informed consent for recipients who face substantial mortality without transplantation. This classification scheme should be updated with new information as data become available.

\section{Considerations Related to Renal Cysts and Renal Cell Carcinoma}

The development of kidney cancer in a patient with a single kidney is very concerning because the surgical treatment of renal tumors may result in loss of function of the remaining kidney. The age-stratified lifetime cumulative incidence of kidney cancer is low. In a matched cohort study of 2119 donors in Ontario Canada (1992-2010) and 21190 nondonors from the general population with similar baseline health, no living kidney donor in the cohort received a partial or total nephrectomy of their remaining kidney during follow-up. ${ }^{342}$

The decision to approve donation in a person with kidney cysts depends on radiographic characteristics (Tables 20 and 21). Because simple (Bosniak I) renal cysts are not associated with increased risk of complications, organ dysfunction, or cancer, simple cysts are not contraindications to kidney donation. Cases of back table excision of small renal cell carcinomas after donor nephrectomy, followed by use of the kidney for transplantation have been reported. ${ }^{345-348}$ Based on review of cancer registry reports, published literature and disease transmission cases reported to the OPTN, a 2011 OPTN DTAC Malignancy Subcommittee

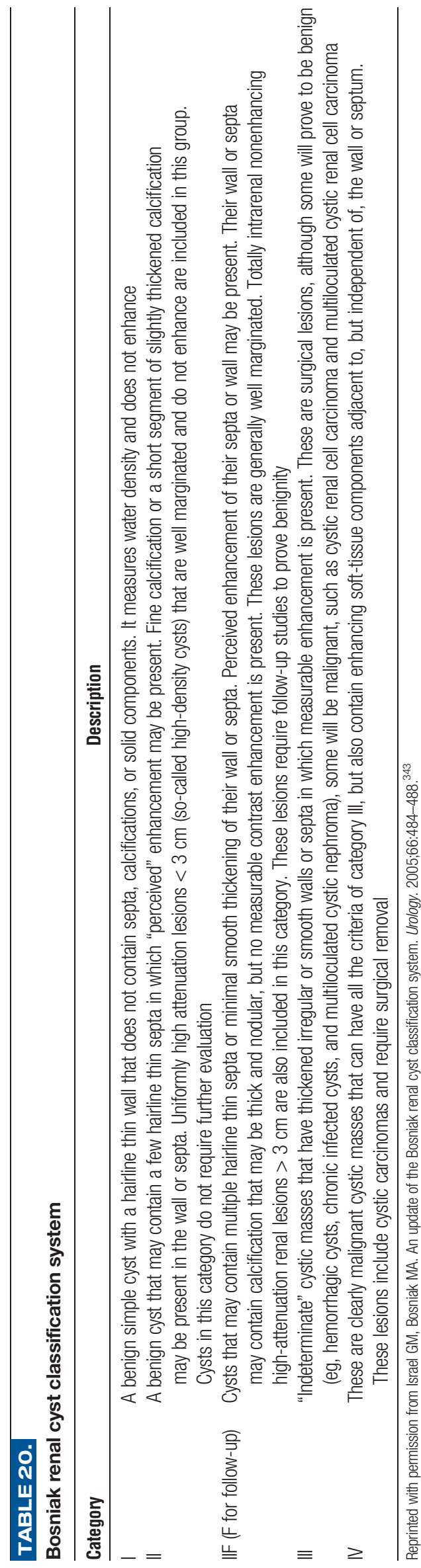


TABLE 21.

International TNM staging system for renal cell carcinoma

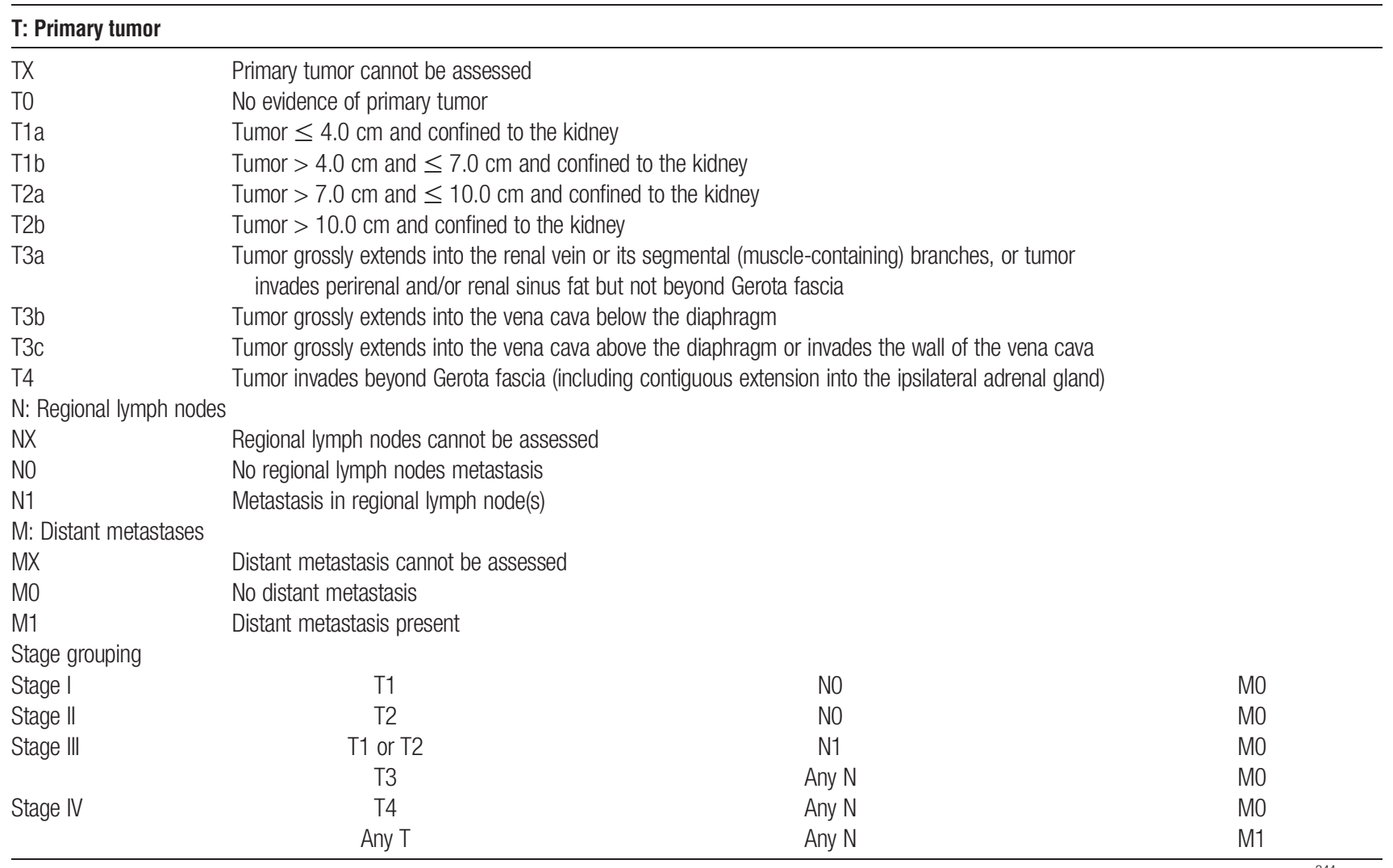

Reprinted from Campbell SC, Lane BR. Chapter 57: Malignant renal tumors. In: Campbell-Walsh Urology, 11th Edn. Wein AJ, Kavoussi LR, Partin AW, et al. (eds). Elsevier, pp. 1314-1364, 2016. ${ }^{344}$

concluded, from a disease-transmission perspective, that "... kidneys with small, solitary, well differentiated renal cell carcinoma may be usable for transplantation provided the lesion itself is completely resected. " ${ }^{341}$ While partial (rather than complete) nephrectomy is often the treatment choice for small renal cell carcinomas for the purpose of nephronsparing with comparable cure rates in affected individuals, persons planning kidney donation intend to undergo complete nephrectomy. Thus, the decision to proceed with donor nephrectomy in an individual with a high grade Bosniak cysts or suspected kidney cancer based on predonation imaging should incorporate considerations of the anticipated risk of future carcinoma in the donor's contralateral kidney, risk of disease transmission to the recipient (including whether the lesion is amenable to complete back table excision), chances of possible discard without transplantation after nephrectomy. Donation of a kidney with a Bosniak II renal cyst should proceed only after assessment for the presence of solid components, septations, and calcifications on the preoperative computed tomography scan (or magnetic resonance imaging) to avoid accidental transplantation of a kidney with cystic renal cell carcinoma. Procurement and transplantation of living donor kidneys with Bosniak (III or higher) renal cysts or small (T1a) renal cell carcinoma curable in the donor by nephrectomy and amenable to complete excision before implantation should proceed only after detailed informed consent of donor and recipient, and donor and recipient understanding and acceptance of these risks. In most circumstances, Bosniak IIF or higher cysts should not be left in the donor but such decision should be individualized.

\section{What Prior Guidelines Recommend}

Prior guidelines and policies for the evaluation and care of living donors recommend history, clinical examination, and investigation to exclude occult malignancy before donation, especially in those older than 50 years or with risk factors including family history. ${ }^{38,48,51}$ The Amsterdam Forum recognized that risks of specific cancers may vary across countries. "Active malignancy" is commonly cited as a contraindication to living kidney donation, ${ }^{38,48,51}$ although exceptions were noted for low-grade nonmelanoma skin cancer. ${ }^{38}$ Renal cell carcinoma was considered a contraindication to donation in some prior guidelines, ${ }^{38,48}$ but is currently permissible under US policy in selected cases. ${ }^{51}$

Past cancers considered to be an absolute contraindication to donation in prior guidelines include melanoma, testicular cancer, choriocarcinoma, hematological malignancy, monoclonal gammopathy, bronchial cancer, and metastatic cancer. $^{38,48}$ Breast cancer is included, although the European Association of Urology qualifies the restriction to "advanced" disease. ${ }^{55}$ Criteria for which donation may be acceptable despite a prior history of malignancy articulated in prior guidelines include that the specific cancer is curable and the potential transmission of the cancer can reasonably be excluded (eg, colon cancer (Dukes A, > 5 years ago), nonmelanoma skin cancer, or carcinoma in situ of the cervix). ${ }^{38,48}$ The Amsterdam Forum defined additional criteria 
for approving donation in a person with prior cancer to include that prior treatment of the malignancy did not decrease renal reserve, place the donor at increased risk for ESKD, or increase the operative risk of nephrectomy. ${ }^{38}$

\section{RESEARCH RECOMMENDATIONS}

- Improve quantification of the incidence of donor-derived disease transmission according to cancer type, clinical features and duration since treatment, through improved monitoring and reporting. Support and expand efforts such as the "Notify Project," 349 a consortium of global experts who gather didactic information on documented types of adverse outcomes in transplantation to identify general principles for detection and investigation.

- Develop systematic monitoring of long-term donor and recipient outcomes in the case of kidney transplantation from living donors with small (T1a) renal cell carcinoma to better inform guidance for when donation and transplantation may or may not be acceptable.

\section{CHAPTER 14: EVALUATION OF GENETIC KIDNEY DISEASE}

The ERT search parameters did not identify evidence from eligible studies pertinent to the recommendations in chapter 14 and therefore the following recommendations are "Not Graded."

\section{Evaluation}

14.1: Donor candidates should be asked about their family history of kidney disease, and when present, the type of disease, time of onset, and extra-renal manifestations associated with the disease.

14.2: When the intended recipient is genetically related to the donor candidate, the cause of the intended recipient's kidney failure should be determined whenever possible. The intended recipient should consent to share this medical information with the donor evaluation team, and with the donor candidate if it could affect the decision to donate.

\section{Selection}

14.3: Donor candidates found to have a genetic kidney disease that can cause kidney failure should not donate.

\section{Counseling}

14.4: Donor candidates must provide informed consent for genetic testing if indicated as part of their evaluation. Donor candidates should be informed of the possible effects of receiving a diagnosis of a genetic kidney disease, such as any impact on their ability to obtain health or life insurance.

14.5: In cases where it remains uncertain whether the donor candidate has a genetic kidney disease and whether the disease can cause kidney failure, donation should proceed only after informing the donor candidate of the risks of donation if the disease manifests later in life.

\section{Autosomal Dominant Polycystic Kidney Disease (ADPKD)}

14.6: Donor candidates with ADPKD should not donate.

14.7: Donor candidates with a family history of ADPKD in a first-degree relative may be acceptable for donation if they meet age-specific imaging or genetic testing criteria that reliably exclude ADPKD.

\section{Apolipoprotein L1 (APOL1) Risk Alleles}

14.8: Apolipoprotein L1 (APOL1) genotyping may be offered to donor candidates with sub-Saharan African ancestors. Donor candidates should be informed that having 2 APOL1 risk alleles increases the lifetime risk of kidney failure but that the precise kidney failure risk for an affected individual after donation cannot currently be quantified.

\section{RATIONALE}

\section{Goals of Evaluation}

Genetic kidney diseases include autosomal dominant polycystic kidney disease (ADPKD), APOL1-related kidney disease, atypical hemolytic uremic syndrome (aHUS), Alport syndrome, Fabry disease, familial focal segmental glomerulosclerosis (FSGS), and autosomal dominant tubulointerstitial kidney disease (ADTKD). A family history of a genetic kidney disease with an autosomal recessive mode of inheritance (such as cystinosis or some forms of familial FSGS) is usually not a contraindication for living kidney donation, although long-term follow-up data are not available in the literature.

All donor candidates should be asked detailed questions about a possible family history of hereditary kidney disease, and all reasonable measures should be taken by health professionals caring for the intended recipient to determine the cause of kidney failure in the setting of possible hereditary kidney disease. With permission of the intended recipient, information about the intended recipient's cause of kidney failure should be reviewed carefully by the donor evaluation team and shared with the donor candidate if it could affect the decision to donate.

Some donor candidates have a family history of genetic kidney disease. ${ }^{350}$ Some of these diseases first manifest later in life and are not identified when donor evaluation occurs at a younger age. Donation could increase the lifetime risk of kidney failure in such a candidate. For this reason, it is important that transplant programs have a strategy for evaluating for inherited kidney disease in donor candidates when there is a family history of kidney failure and the recipient's cause of kidney failure is uncertain.

Many of the standard tests done as part of the donor evaluation should be interpreted with special consideration in the setting of a family history of genetic kidney disease. Examples include renal imaging in the setting of family history of ADPKD, or hematuria testing in the setting of a family history of Alport syndrome (see also chapter 7).

Ideally, the estimation of long-term postdonation risk in a donor candidate should account for their family history of genetic kidney disease, but there are currently no tools that incorporate family history of genetic kidney disease with a donor candidate's other demographic and health characteristics to estimate long-term risk. Living donors who are biologically related versus unrelated to a recipient may have a higher incidence of ESKD. In a large study from the United States, the 15 -year cumulative incidence of ESKD was $0.34 \%$ in living donors who were biologically related to a recipient versus $0.15 \%$ in unrelated donors (a two-fold increase in relative terms, although difference was not statistically significant $(P=0.15) .{ }^{30}$

Donor candidates must provide informed consent to have genetic testing if indicated as part of their evaluation. Donor 
candidates should be informed of the possible effects of receiving a diagnosis of a genetic kidney disease, such as any effects on their ability to obtain health or life insurance. In cases where despite a normal evaluation at the time of donor candidate evaluation it remains uncertain whether the candidate has a genetic kidney disease, donation should proceed only after a full discussion with the donor candidate of the risks of donation if the disease manifests later in life.

With advances in genetic medicine and the implications of new risk alleles such as APOL1, there is likely to be rapidly evolving knowledge that may influence future donor candidate evaluations. However, at this time the testing for several genetic conditions is imperfect. This uncertainty should prompt a discussion with all relevant stakeholders to achieve consensus on proceeding with donation or not.

\section{Autosomal Dominant Polycystic Kidney Disease (ADPKD)}

Recent studies and a KDIGO Controversies Conference summarize diagnostic criteria for ADPKD. ${ }^{351,352}$ Simple cysts occur more frequently with increasing age in the general population. Kidney disease from $P K D 2$ presents later in life than PKD1. Age-dependent imaging criteria for diagnosis and disease exclusion have been established for at-risk adults with unknown ADPKD gene type (PKD1 or PKD2). ${ }^{353}$ For example, for candidates 40 years or older with a first degree relative with $\mathrm{ADPKD}$, the finding of normal kidneys or a single cyst seen in one kidney with no cysts seen in the other kidney reliably rules out the presence of ADPKD. ${ }^{354}$ However, the utility of ultrasound to rule out ADPKD in younger donor candidates is more limited, where an absent or limited number of cysts on CT or MRI can be considered to rule out ADPKD. ${ }^{352}$ DNA testing can also sometimes help diagnose or exclude the condition. ${ }^{355}$ Linkage-based genetic diagnoses of ADPKD using polymorphic markers flanking the 2 disease genes is now rarely performed. Rather, direct mutation screening (by Sanger or next-generation sequencing) is now commonly used for molecular diagnosis of ADPKD. ${ }^{356,357}$ Up to $15 \%$ of patients with suspected ADPKD have a negative comprehensive mutation screen. The first-degree relative with ADPKD should undergo PKD1 and PKD2 mutation screening (using an acceptable technique), and if a pathogenic mutation is successfully identified, the donor candidate can be tested for this same mutation. However, when mutation screening in the first-degree relative with ADPKD is negative, DNA testing including molecular diagnostics is unhelpful in determining whether the donor candidate does or does not have ADPKD.

The criteria to diagnose ADPKD in patients with no family history of ADPKD is less certain (a family history is absent in $10-15 \%$ of patients with ADPKD). A patient with bilaterally enlarged kidneys with innumerable cysts most likely has ADPKD, although the presence of other cystic kidney diseases should also be considered. ${ }^{351}$

\section{Apolipoprotein L1 (APOL1) Risk Alleles}

The use of APOL1 genotyping in the donor evaluation is currently grounded primarily in evidence extrapolated from nondonor populations. Recent literature supports that at least a portion of kidney failure previously attributed to hypertensive nephrosclerosis or other diseases (eg, FSGS, lupus nephritis, HIV nephropathy, sickle cell disease) in persons of
African descent may be genetically mediated or accelerated by coding variants in the APOL1 gene. Having at least one APOL1 risk allele confers resistance to lethal Trypanosoma brucei infections, and these variant alleles are observed in populations of African descent but essentially absent among white persons. Regionally, APOL1 risk variants are common in persons from West Africa and South Africa (sub-Saharan African descent), and uncommon in North Africa. Approximately $13 \%$ of African Americans carry 2 APOL1 risk alleles. ${ }^{358}$ Among African Americans in the general population, carrying 2 APOL1 risk alleles has been associated with FSGS ${ }^{359}$ and HIV-associated nephropathy histopathologies, proteinuria, reduced GFR, younger age at dialysis and more rapid progression of kidney disease. ${ }^{235-238}$ The presence of 2 APOL1 risk alleles in a deceased donor associates with 2-to-4-times higher RR of graft loss in the recipient, compared with 0 or 1 risk alleles. ${ }^{360,361}$ Case reports of possible APOL1mediated adverse donor and recipient outcomes after living kidney donation among siblings of African ancestry were recently described. ${ }^{359,362}$

Routine use of APOL1 genotyping and related counseling within evaluation protocols has been advocated by some ${ }^{358,363-365}$ while others raise concerns. ${ }^{366}$ The utility of APOL1 testing has not been addressed in prior living donor guidelines. The impact of APOL1 screening on donor candidate exclusion, and the impact of test results on postdonation outcomes and graft outcomes in living donor transplant recipients are yet to be defined. ${ }^{367}$ Nevertheless, given APOL1-related risks identified in the general population and among deceased donor transplant recipients, as well as known higher risk of renal complications among African American compared with white donors, ${ }^{368}$ we recommend considering APOL1 genotyping in donor candidates with sub-Saharan African ancestors. The implications of having 2 APOL1 risk alleles likely differ in younger versus older donor candidates, and whether the genetically related intended recipient has the same alleles. The implications of having 2 APOL1 risk alleles may also be influenced by the results of kidney function testing done at the time of donor candidate evaluation.

A 2016 AST Expert Conference opined that pending more evidence, at this time all African American living donor candidates should be informed about the associations of APOL1 risk alleles with CKD/ESKD risk in the general population and offer genetic testing as part of the evaluation (including coverage of testing costs) to candidates who wish to know their APOL1 status. ${ }^{369}$ The WG also recommended counseling donor candidates that precise ESKD risk for an affected individual cannot currently be quantified, and that genetic testing may post psychological stress. Finally, the authors advised that use of APOL1 risk allele status should be integrated with the donor candidate's full profile of demographic and health characteristics in determining donor candidacy.

\section{Alport Syndrome (See also Chapter 7)}

Alport syndrome is a genetic disease that alters collagen biosynthesis. Collagen is an important structural component of the basement membranes in the kidney, inner ear and eye, and the clinical manifestations of Alport syndrome include persistent microscopic hematuria, early onset bilateral sensorineural hearing loss, and ocular anterior lenticonus and 
retinal flecks. The combination of a detailed family history assessment and a kidney biopsy helps establish the diagnosis, where electron microscopy demonstrates areas of irregular glomerular basement membrane thickness with lamellation, splitting and sometimes characteristic "basket-weave appearances." ${ }^{370}$ The presence of Alport syndrome can be confirmed by genetic testing, and next-generation sequence panels provide opportunities to genetically screen for collagen mutations. ${ }^{371}$ Alport syndrome is primarily an X-linked disorder (approximately $80 \%$ of families), but can also be inherited in an autosomal recessive (approximately 15\% of families) and autosomal dominant fashion (very rare). ${ }^{372,373}$ Most women who are heterozygotes for X-linked Alport syndrome exhibit hematuria (95\% of women in one European cohort). Of note, collagen (COL4A) mutations are present in families with Alport syndrome (85\% of Alport syndrome is caused by mutations of COL4A5), and are also present in some families with familial FSGS, sporadic FSGS, and thin basement membrane disease. ${ }^{374,375}$ Overall, the severity of nephropathy in Alport syndrome is variable. Many males with X-linked Alport syndrome will develop ESKD before the age of 40 years. Most female carriers of X-linked Alport syndrome will not develop kidney failure in their lifetime although cases of ESKD do occur. ${ }^{370,373}$

There is little information on the outcomes of such heterozygotes who proceeded with kidney donation (after confirming the absence of proteinuria, hypertension, low GFR and other manifestations of the disease such as sensorineural hearing loss). Gross et a ${ }^{174}$ described 6 mothers with Alport syndrome (across several European centers) who donated kidneys to their children with the disease. Five mothers with $\mathrm{X}$-linked Alport syndrome donated to their sons and one mother who was a carrier of autosomal recessive Alport syndrome donated to her daughter; kidney function declined $25 \%$ to $60 \%$ in 4 of the 6 donors over the observed 2 to 14 years after nephrectomy, although no donor's creatinine clearance was less than $40 \mathrm{~mL} / \mathrm{min}$ at the time of follow-up evaluation; 4 of the 6 developed microalbuminuria or proteinuria, and hypertension was diagnosed in 4 of 6 donors. There are often several considerations in evaluating a mother with X-linked Alport syndrome for donation to a son with kidney failure, including her desire to care for her child and the possible guilt associated with passing on a genetic kidney disease. ${ }^{376}$ Alternative treatment options should be considered (including other living donors), as should the age of the woman (older women have had more time to manifest kidney disease, so normal testing at the time of their evaluation is more reassuring than similar results in a younger woman).

\section{Fabry Disease}

Fabry disease is an X-linked lysosomal storage disease caused by deficiency of the lysosomal hydrolase, $\alpha$ galactosidase A ( $\alpha$-Gal A), which results in systemic accumulation of trihexosylceramide (globotriaosylceramide [GL-3]) in the lysosomes of the vascular endothelium in multiple organs. Clinical features include neuropathic pain and angiokeratoma, proteinuria, CKD, left ventricular hypertrophy, arrhythmia and stroke. Symptoms generally appear in childhood, although some go unrecognized until adulthood. Many affected males develop ESKD by the time they are 35 to 45 years of age. Heterozygous females have a different clinical course with variable clinical manifestations owing to random X chromosome inactivation. Renal manifestations include microscopic hematuria and the presence of white blood cells in the urine; less than $1 \%$ of heterozygous females develop kidney failure in their lifetime. A presumptive clinical diagnosis of Fabry disease can be made on the basis of a family history and signs and symptoms, with confirmatory testing for deficient $\alpha$-galactosidase A enzyme activity in plasma and leukocytes (this deficiency is evident in males with the disease), and genetic testing. ${ }^{377}$

There is very little information on the outcomes of heterozygous females with Fabry disease who proceeded with kidney donation. ${ }^{378}$ As with heterozygous Alport syndrome carriers, if donation is entertained, considerations should include the age of the woman and careful deliberation of all other treatment options for the intended recipient.

\section{Familial Focal Segmental Glomerulosclerosis (FSGS)}

In recent years, many inheritable genetic forms of FSGS have been described, caused by mutations in a number of genes for proteins that are important for podocyte function or glomerular basement membrane assembly. ${ }^{374,379}$ FSGS can follow both recessive and dominant inheritance patterns. ${ }^{380}$ Of note, other clinical terms are used to describe the same or similar diseases based on age of onset or response to therapy (eg, congenital nephrotic syndrome, steroid resistant nephrotic syndrome). ${ }^{381}$

There are case reports of individuals who have developed FSGS, proteinuria and kidney failure after donating a kidney to a sibling with kidney failure from FSGS. ${ }^{382}$ The role of genetic testing in relatives with a family history of kidney failure from FSGS is yet to be determined.

\section{Atypical Hemolytic Uremic Syndrome (aHUS)}

There are case reports of individuals who donated a kidney to a relative with kidney failure from Atypical Hemolytic Uremic Syndrome (aHUS), where the donor developed aHUS in the year after donation. ${ }^{383} \mathrm{~A}$ high chance of graft failure from aHUS reoccurrence in the recipient has also been described (>80\% with some aHUS mutations). Current genetic testing is imperfect in excluding the presence of aHUS in a donor candidate even when the mutation is known in the recipient. For these reasons some suggest never to proceed with living kidney donation in the setting of a related recipient with aHUS. Others suggest assessing whether a donor candidate shares a genetic susceptibility factor to aHUS to determine whether or not they may be an acceptable donor. ${ }^{384}$

\section{Autosomal Dominant Tubulointerstitial Kidney Disease (ADTKD)}

ADTKD is a heterogeneous genetic disorder. Individual families may have a large number of affected individuals. Mutations in at least 4 genes are implicated: MUC1 gene which encodes mucin 1 (MCKD1), REN gene which encodes renin, UMOD gene which encodes uromodulin (MCKD2) and the HNF1B gene which encodes hepatocyte nuclear factor- $1 \beta .{ }^{385,386}$ Similar to other autosomal dominant diseases, there is $50 \%$ probability that each child will inherit the disease from their affected parent. Both HNF1B and MUC1 mutations can arise de novo without a prior family history of disease. Donor candidates may be offered target mutation screening to assess whether the disease is present, 
if they are biologically related to a patient with kidney failure who has evidence of the pathogenic mutation.

\section{Sickle Cell Trait}

Approximately $8 \%$ of black Americans have sickle trait, and the trait is also common among patients with Mediterranean or Indian subcontinent heritage. ${ }^{387,388}$ Many affected persons may be unaware that they carry the trait. The prevalence of sickle trait among potential and actual kidney donors is not known. Renal abnormalities in the presence of sickle cell trait range from isosthenuria to hematuria, to rare presentations with acute kidney injury in the context of severe physical stress, such as military training or pregnancy. ${ }^{389}$ The manifestations of sickle cell trait depend on the individual's overall hemoglobin genotype and exposure to environmental stressors. ${ }^{389,390}$ An increased incidence of medullary renal carcinoma has also been reported among persons with sickle cell trait. A prior survey of US transplant programs (2005, 54\% response rate) demonstrated substantial variation in screening and exclusion practices, such that $83 \%$ $(113 / 137)$ of responding programs did not have a policy to screen donors for sickle cell trait. ${ }^{391}$ Among the programs reporting related exclusion practices, 18\% (19/105) reported always excluding donor candidates with sickle cell trait whereas $16 \%$ (17/105) indicated they never exclude.

\section{What Prior Guidelines Recommend}

Prior guidelines and policies for the practice of living kidney donation discuss genetic considerations in the evaluation, although not to the extent presented in the current guideline. Current OPTN policy requires that US transplant programs establish and follow a protocol for screening for ADPKD and other inherited kidney diseases as guided by family history. ${ }^{51}$ Several guidelines including those from the British Transplantation Society describe imaging criteria used to exclude the presence of ADPKD. ${ }^{48}$

\section{RESEARCH RECOMMENDATIONS}

- Determine how a genetic predisposition to various kidney diseases relates to outcomes after donation. Use genetic information along with other candidate characteristics to estimate the longterm risk of ESKD in the absence and presence of donation.?

- Develop better strategies and tools to screen donor candidates for genetic kidney diseases that consider the accuracy, efficiency and costs of testing, including assessment of targeted gene panels for known mutations implicated in kidney diseases. ${ }^{392}$

- Develop reliable imaging criteria to exclude ADPKD in donor candidates based on the results of the CT angiogram, which is frequently used to assess the renal vasculature as a routine part of the donor evaluation.

- Define the role APOL1 genotyping in the evaluation of donor candidates of sub-Saharan African ancestry. ${ }^{369}$ Acquire adequate data to assess rare outcomes such as ESKD in donors from national registries incorporating biospecimens and long-term outcomes information.

- For example, in December 2016 the NIH announced 2 requests for applications to form the APOL1 Long-term Kidney Transplantation Outcomes (APOLLO) Network consortium directed at designing and conducting multicenter, prospective, longitudinal studies to determine the impact of APOL1 genetic variants on outcomes after living kidney donation/transplantation and deceased donor kidney transplantation. 393,394
- Better define the implications of sickle cell trait on long-term kidney function and implications for donor selection.

\section{CHAPTER 15: PREGNANCY}

Except in the case of Recommendation 15.9, the ERT search parameters did not identify evidence from eligible studies pertinent to the recommendations in chapter 15 and therefore the following recommendations are "Not Graded."

\section{Evaluation}

15.1: Female donor candidates should be asked about future childbearing plans.

15.2: Female donor candidates should be asked about prior hypertensive disorders of pregnancy (eg, gestational hypertension, preeclampsia, or eclampsia).

15.3: Local guidelines should be followed to confirm the absence of pregnancy before performing radiologic tests, including abdominal computed tomography (with iodinated contrast) or nuclear medicine GFR testing.

\section{Selection}

15.4: Women should not donate while pregnant.

15.5: Women should not be excluded from donation solely because they desire to conceive children after donation.

15.6: Women with a prior hypertensive disorder of pregnancy may be acceptable for donation if their longterm postdonation risks are acceptable.

15.7: A decision to proceed with donation in the year after childbirth should consider the psychological needs of mother and child, and should include anesthesia and analgesia planning for nursing mothers.

\section{Counseling}

15.8: Women with childbearing potential should be informed of the need to avoid becoming pregnant from the time of approval for donation to the time of recovery after nephrectomy; a quantitative human chorionic gonadotropin $(\beta-h C G)$ pregnancy test should be performed and confirmed as negative immediately before donation.

15.9: We suggest that women with childbearing potential be counseled about the effects donation may have on future pregnancies, including the possibility of a greater likelihood of being diagnosed with gestational hypertension or preeclampsia. (2C)

15.10: Women with a prior hypertensive disorder of pregnancy should be informed about their long-term risks.

15.11: Women with childbearing potential who proceed with donation should be counseled on how to reduce the risk of complications in future pregnancies.

\section{RATIONALE}

\section{Evaluation and Selection}

Female donor candidates should be asked about their future childbearing plans and potential, which is determined by the candidate's age, history of menopause, and any prior history of sterilization. This information has implications for counseling and the need to confirm the absence of pregnancy at the time of donor nephrectomy.

When evaluating donor candidates, knowledge about a prior history of hypertensive disorder during pregnancy and its severity is important, as when such a history is present 
(vs absent) it is associated with a higher risk of ESKD. ${ }^{395}$ One meta-analysis concluded that women were at greater risk of albuminuria after a preeclamptic pregnancy compared with a normal pregnancy (5- to 10-year incidence of $31 \%$ vs $7 \%) .{ }^{396}$ This meta-analysis was, however, restricted by small studies of variable quality that focused often on women with severe preeclampsia or underlying diseases such as diabetes mellitus. A population-based study from Norway suggested that women were at greater risk of ESKD if they developed preeclampsia during pregnancy than if they did not (approximate 30-year cumulative incidence after a woman's first pregnancy of $0.4 \%$ vs $0.1 \%)^{397}$ The risk remained evident after excluding women with a prepregnancy history of known kidney disease, diabetes or hypertension. A study from Taiwan also described a greater risk of ESKD in pregnant women who developed a hypertensive disorder during pregnancy compared with a normal pregnancy (approximate 12 -year cumulative incidence after pregnancy of $0.6 \%$ vs < $0.1 \%)^{398}$ In general, characteristics associated with a lower long-term risk of ESKD after a history of hypertension in pregnancy include: i) a mild (vs severe) hypertensive event during pregnancy; ii) more than 10 years since last hypertension event in pregnancy; iii) no evidence of hypertension, albuminuria or low GFR in the current donor evaluation; and iv) no wish for future pregnancies.

Gestational diabetes is a strong risk factor for subsequent diabetes mellitus. A history of gestational diabetes mellitus is also associated with a higher risk of ESKD. ${ }^{399}$ For recommendations related to gestational diabetes see Recommendations 11.5 and 11.7.

Because a fetus may be harmed by radiation and/or or radiocontrast, local guidelines should be followed to confirm the absence of pregnancy before performing certain tests as part of the donor candidate evaluation, such as an abdominal CT (with iodinated contrast) or a nuclear GFR test. Some guidelines suggest that it may be reasonable to proceed without a quantitative human chorionic gonadotropin ( $\beta-\mathrm{hCG}$ ) pregnancy test if a woman's menstrual period is not overdue and there is an absence of pregnancy symptoms. ${ }^{400}$

A woman should not donate while pregnant. Women with childbearing potential should be informed about the need to avoid becoming pregnant from the time she is approved for donation to the time she has recovered after nephrectomy. Approximately $1 \%$ of patients develop postoperative venous thromboembolism after donor nephrectomy and the risk of using estrogen-based oral contraceptive medications before surgery should be balanced against harms. Barrier birth control can be an appropriate option in the weeks before surgery.

Women with childbearing potential should be supported by transplant programs to make a well-informed donation decision. Transplant programs should enable donation when they estimate a candidate's long-term postdonation risks are acceptable. In other words, motivated well-informed women should not be excluded from donation solely on the basis of a desire to conceive children after donation. Most women with access to recommended pregnancy care have good maternal and fetal outcomes in their postdonation pregnancies. ${ }^{401} \mathrm{We}$ advocate that recommended postdonation pregnancy care be available to all women worldwide, including any treatments needed for pregnancy complications. A decision to proceed with donation in the year after delivery should carefully consider the needs of the mother and her baby.
With regard to prior guidelines, the 2004 Amsterdam Forum concluded that donor nephrectomy is not detrimental to the prenatal course or outcome of future pregnancies. At the time, the participants concluded there were no data to suggest that hyperfiltration associated with the combination of unilateral nephrectomy and pregnancy leads to significant hypertension, proteinuria, change in GFR, or abnormalities of the urinary sediment. ${ }^{38}$ Some transplant programs have historically not disclosed any potential postdonation pregnancy risks as part of the informed consent process. However, 3 recent studies provided new information on this risk. Two retrospective cohort studies, one from the United States and the other from Norway, reported an increased risk of gestational hypertension and preeclampsia after kidney donation, based on comparisons of pregnancies before and after donation within donors (Evidence Report Table 21, SDC, http://links.lww.com/TP/B434, and Supplemental Appendix Table D18, SDC, http://links.lww.com/TP/B432). ${ }^{402,403}$ Another recent retrospective cohort study from Canada demonstrated gestational hypertension or preeclampsia was more likely to be diagnosed in kidney donors than matched nondonors with similar indicators of baseline health $(11 \% \text { vs } 5 \%)^{401}$ The 2 groups did not differ significantly with respect to other maternal or fetal outcomes (Caesarean section, postpartum hemorrhage, preterm birth with gestation less than 37 weeks, low birth weight $<2500 \mathrm{~g}$ ), and there were no maternal deaths, stillbirths, or neonatal deaths in either group (however, the CIs for the estimates were wide, meaning that a clinically important risk among donors was not excluded). Most women in these cohorts were white (Table 22).

\section{Counseling}

Based on the new information, our guideline suggests that women who are capable of conceiving children after donation be counseled about the possible impact donation may have on future pregnancies. This suggestion agrees with a 2015 AST Live Donor Community of Practice consensus conference conclusion that information on postdonation pregnancy risk should be shared in the informed consent process for donor candidates with reproductive potential. ${ }^{148} \mathrm{Be}-$ ginning in 2017, OPTN Informed Consent Policy requires that US transplant programs inform donor candidates that the risks of preeclampsia or gestational hypertension are increased in pregnancies after donation. ${ }^{51}$ The ERT found the evidence for a higher likelihood of being diagnosed with gestational hypertension or preeclampsia after donation to be consistent across the 3 studies, but of low quality. ${ }^{3}$

Women with childbearing potential who proceed with donation should be educated on recommended evidence-based methods to reduce the risk of complications in postdonation pregnancies. For example, in the nondonor population, several dietary and lifestyle interventions including interventions to reduce or prevent obesity have the potential to reduce the risk of preeclampsia. ${ }^{404,405}$

\section{RESEARCH RECOMMENDATIONS}

- Investigate the precision of estimated, individualized pregnancy outcomes among donors through expanded monitoring and reporting, including capture of more detailed clinical information such as BP and kidney function. 


\section{TABLE 22.}

Donor characteristics and maternal and fetal outcomes in postdonation pregnancies from 3 studies: Norway, Minnesota (United States), and Ontario (Canada)

\begin{tabular}{|c|c|c|c|}
\hline & Norway & Minnesota, United States & Ontario, Canada \\
\hline \multicolumn{4}{|l|}{ Donor characteristics } \\
\hline No. women & 69 & 239 & 85 \\
\hline Years of donation & 1967 to 2002 & 1963 to 2007 & 1992 to 2009 \\
\hline Family history of kidney failure (\%) & NR & $96 \%$ & $65 \%$ \\
\hline Mean predonation GFR, mL/min per $1.73 \mathrm{~m}^{2}$ & $N R$ & 91 & 114 \\
\hline White race $(\%)$ & $98 \%$ & $97 \%$ & $70 \%$ \\
\hline Number of postdonation pregnancies & 106 & 490 & 131 \\
\hline Mean age at donation, y & 27 & 26 & 29 \\
\hline Mean age at pregnancy, y & 32 & 29 & 32 \\
\hline No. women with at least one predonation pregnancy (\%) & NR & $98(41 \%)$ & $25(29 \%)$ \\
\hline Mean or median time from donation to postdonation pregnancies, y & 5 & 5 & 4 \\
\hline \multicolumn{4}{|l|}{ Outcomes after donation } \\
\hline \multicolumn{4}{|l|}{ Postdonation maternal outcomes } \\
\hline Gestational hypertension or preeclampsia & 9/106 (8\%) & $55 / 490(11 \%)$ & $15 / 131(11 \%)$ \\
\hline Gestational hypertension & $3 / 106(3 \%)$ & $28 / 490(6 \%)$ & $7 / 131(5 \%)$ \\
\hline Preeclampsia & 6/106 (6\%) & $27 / 490(6 \%)$ & $8 / 131(6 \%)$ \\
\hline Maternal death & NR & $\mathrm{NR}$ & 0 \\
\hline \multicolumn{4}{|l|}{ Postdonation fetal outcomes } \\
\hline Preterm birth (<37 wk gestation) & $10 / 106(9 \%)$ & $35 / 490(7 \%)$ & $10 / 131(8 \%)$ \\
\hline Low birth weight $(<2500 \mathrm{~g})$ & 9/106 (8\%) & NR & 8/131 (6\%) \\
\hline Stillbirth & $3 / 106(3 \%)$ & $2 / 490(<1 \%)$ & 0 \\
\hline Neonatal death (<28 days after birth) & $0(0.0 \%)$ & $\mathrm{NR}$ & 0 \\
\hline
\end{tabular}

GFR, glomerular filtration rate; NR, not reported. Modified with permission from Garg AX et al. ${ }^{401}$

- Develop individualized estimates for women who differ in race/ethnicity, family history of kidney disease, and their healthcare system.

- Determine whether the risks of complications in postdonation pregnancies vary according to the side of nephrectomy. Pregnancy may be associated with ureteral obstruction and dilation, particularly of the right kidney. ${ }^{37,406,407}$

- Define the clinical sequelae of gestational hypertension and preeclampsia that develop in postdonation pregnancies, including effects on long-term kidney outcomes.

- Explore perceptions of pregnancy risk information by women with different characteristics to improve education, counseling, and informed consent processes.

\section{CHAPTER 16: PSYCHOSOCIAL EVALUATION}

Except in the case of Recommendation 16.7, the ERT search parameters did not identify evidence from eligible studies pertinent to the recommendations in chapter 16 and therefore the following recommendations are "Not Graded.”

\section{Evaluation}

16.1: Donor candidates should receive in-person psychosocial evaluation, education and planning from health professionals experienced in the psychosocial concerns of donor candidates and donors.

16.2: To ensure voluntariness, at least a portion of the psychosocial evaluation of the donor candidate should be performed in the absence of the intended recipient, family members and other persons who could influence the donation decision.
16.3: Whenever possible, the psychosocial evaluation of the donor candidate should be performed by health professionals not involved in the care of the intended recipient.

16.4: Transplant programs should follow protocols for assessing the donor candidate's psychosocial suitability, available support, preparation and concerns for donation.

\section{Selection}

16.5: Transplant programs should follow protocols defining psychosocial factors that either exclude donation, or prevent further evaluation until resolution.

\section{Disclosures and Support}

16.6: We suggest that donor candidates be informed that donors usually have good quality of life after donation (2D).

16.7: Transplant programs should assist donor candidates and donors in receiving psychosocial or psychiatric support as needed.

\section{RATIONALE}

\section{Evaluation}

The psychosocial evaluation of the living kidney donor candidate serves many functions. This evaluation helps determine if a candidate is psychologically fit for donation, for example, by assessing if the donor candidate's motivations are appropriate and identifying psychosocial risk factors for poor outcomes. The evaluation also helps address any donor candidate concerns, and ensures potential psychosocial risks and benefits of kidney donation are disclosed and understood. The psychosocial evaluation can also be used to 
develop a tailored plan to support the donor candidate in having a positive psychosocial experience throughout the evaluation and donation processes. We recommend that all donor candidates, irrespective of their relationship with the intended recipient, should be evaluated using uniform criteria.

A recent systematic review of living kidney and liver donor candidates summarized 34 publications (guidelines, consensus statements and transplant program clinical protocols) on the content of the psychosocial evaluation (including the elements to be considered and acceptance criteria) and the process of the psychosocial evaluation (including who should be evaluated, how the evaluation should be performed, and the timing of the evaluation). ${ }^{408}$ The authors concluded that, at present, there is little good evidence, concrete guidance or consensus on what to screen for, how to handle psychosocial problems, or how to perform the psychosocial evaluation. These findings confirmed that there is substantial practice variation in regard to psychosocial evaluations. The authors concluded that, consistent with prior systematic reviews, ${ }^{20}$ most recommendations in prior publications are based on expert opinions (via consensus conference) and individual transplant program experiences. The ERT assessed evidence related to psychosocial outcomes after donation.

\section{Should all Donors have a Psychosocial Evaluation?}

Our recommendations are consistent with several prior guidelines and policies in some countries, which indicate that psychosocial assessment is a necessary part of the evaluation of each donor candidate. ${ }^{38,48,50,51,54,55,409}$

\section{In What Setting Should the Evaluation Be Performed?}

While the initial screening may be performed by telephone, particularly for donor candidates living far from the transplant center, the main psychosocial evaluation should be conducted as a face-to-face interview, as has also been recommended in several prior reports. ${ }^{408}$ Third parties should not be present during at least a portion of the interview, to maintain confidentiality and minimize the effect of external influences on the donor candidate's responses. This suggestion is consistent with some prior reports. ${ }^{408}$ In other reports the presence of a relative or significant other during a portion of the interview (as long as they are not the intended recipient) is cited as potentially beneficial to the donor candidate and the assessment process, in that such participation may promote reliability of the information reported and provide information about support the donor candidate will need during the donation and recovery periods. ${ }^{408}$

\section{Who Should Perform the Psychosocial Evaluation?}

Our recommendations are similar to many prior guidelines and national policies that recommend performance of the psychosocial evaluation by various healthcare professionals (eg, psychiatrist, psychologist, clinical social worker, or a psychiatric nurse) who meet qualifications of appropriate training, knowledge and skill in mental health and the psychosocial concerns of transplantation. ${ }^{51,54}$ As with prior recommendations we suggest that the psychosocial evaluation should be performed by an individual who is not involved in the care of the intended recipient to reduce conflicts of interest in evaluating the donor candidate. ${ }^{50}$

\section{TABLE 23}

Recommended processes and content of the psychosocial evaluation

Process Content element

Assessment of suitability and preparation The following should be assessed in each donor candidate:

- Motivation for donation

- Expectations of the outcomes of donation, including impacts on their future relationship with the recipient. The donor candidate should be counseled if these expectations appear to be unrealistic.

- History of current or past psychiatric disorders

- History of current or past substance abuse or dependence (eg, use of alcohol, illicit drugs or prescription drugs)

- Support system, including available family, friends and workplace supports, and coping strategies (including available programs) to minimize any negative impacts from donation

- Preparation for the possible medical impact of donation, and any expected impact on their activities after donation

- Preparation for the possible emotional impacts of donation (both positive and negative)

- Preparation for the possible financial impacts of donation, including potential impact on employment status and wages, expenses for medical care, travel and dependent care, and any effects on insurability or rates

- Understanding and acceptance of their responsibility for making good health choices after donation, including the need to organize and adhere to recommended routine medical follow-up

- Behaviors that increase the risk of a donor transmitting an infectious disease to the recipient

Education

- The process and requirements of informed consent should be reviewed (see chapter 2 of this guideline for complete details)

- Concerns about medical, emotional and financial impacts of donation should be elicited and addressed with appropriate education and/or referral to a knowledgeable member of the transplant team

- Donor candidates can be told that most prior donors have experienced good psychosocial outcomes, although some people experience psychosocial difficulties after donation

Planning and support

- An individualized plan to support the donor candidate in having a positive psychosocial experience throughout the evaluation and donation processes should be developed for each candidate, guided by donor candidate concerns and psychosocial risk factors that can be managed without excluding donation 


\section{Protocols for Assessing the Donor Candidate's Psychosocial Suitability}

It is important for transplant programs to follow predetermined protocols in evaluating each donor candidate's psychosocial suitability equitably. The protocol should include a comprehensive list of the processes and content of the psychosocial evaluation (Table 23). Recommended components of the psychosocial evaluation specified in prior guidelines include mental health history, ${ }^{47,48,51,54,196,409}$ substance use history, ${ }^{47,48,51,54,409}$ understanding of risks, ${ }^{47,51,55}$ ability to make an informed choice, ${ }^{51}$ coping capacity, ${ }^{47,51,409}$ social support, ${ }^{47,48,51,409}$ risk factors for poor psychosocial outcomes (details unspecified), ${ }^{51,409}$ motivation and voluntariness, ${ }^{47,51,409}$ and donor-recipient relationship. ${ }^{47,48}$

\section{Considerations for Nondirected Donor Candidates}

Practice patterns related to the psychosocial evaluation of nondirected donors vary considerably worldwide. ${ }^{410-412}$ Our opinion is that candidacy criteria should be similar whether an individual is being assessed for directed or nondirected donation, recognizing that some elements of the informed consent may vary, including those related to how the intended recipient is selected, time frames for donation, travel requirements, and opportunities or prohibitions about contact with the recipient after donation and transplant.

\section{Selection}

Which Predonation Characteristics are Associated with a Greater Chance of Poor Psychosocial Outcomes after Donation?

The ERT identified 2 studies examining whether the association between donation (donors vs healthy nondonors) and long-term psychological outcomes were modified by an individual's baseline characteristics (Evidence Report Tables 9 and 11, SDC, http://links.lww.com/TP/B434; Supplemental Appendix Tables D6 and D8, SDC, http://inks.lww.com/TP/B432). The quality of this evidence was graded as very low. In one study the association between donation (donors vs nondonors) and long-term mental quality of life score was not modified by age. ${ }^{413}$ In the other study, the cumulative incidence of recorded depression diagnoses was lower in donors compared with matched beneficiaries in the same insurance system, among both men and in women. ${ }^{414}$

The ERT summarized the results of several studies wherein donors with a characteristic were compared to donors without the characteristic, to see if the 2 groups differed on the chance of a poor psychosocial outcome. The quality of this evidence was graded as low to very low. These results are challenging to interpret because: many studies reported different psychosocial outcomes that were not directly comparable; the effects were not always seen across all psychosocial outcomes measured within a study; the results of characteristics associated with outcomes were inconsistent across the studies; and the likelihood a donor with the characteristic would experience the outcome was often reported in relative rather than absolute terms (see Evidence Report Tables 10, 12, 14, 15, 20, SDC, http://links.lww.com/TP/B434; Supplemental Appendix Tables D7, D9, D11, D12, D17, SDC, http://links.lww.com/TP/B432).

In some studies, predonation characteristics that were associated with reduced chance of poor psychosocial outcomes (ie, with a better psychosocial prognosis) included a lack of residual ambivalence about donation (ie, no lingering hesitation or uncertainty), more education, an absence of predonation psychiatric diagnoses, an absence of feeling morally obligated to donate, more social support, lower predonation expectations of health consequences after donation, less financial burden, and the feeling of having received adequate attention from the transplant team. ${ }^{415-419}$ A systematic review of prospective studies of postdonation healthrelated quality-of-life (HRQoL) published after completion of the ERT's systematic review for this guideline found that, among 9 studies examining risk factors for impaired HRQoL, low psychological functioning before donation was the most common predictor. ${ }^{420}$

\section{What Psychosocial Criteria Should Exclude Donation?}

Psychosocial elements that should be reviewed with the donor candidate and the psychosocial criteria that either exclude donation, or prevent further evaluation of the donor candidate until the issue is resolved, are summarized in Table 24. Donation should not occur when there is evidence of, or expectation of, unreasonable secondary gain. The Declaration of Istanbul endorses measures to protect individuals from the unregulated sale of organs. ${ }^{421}$ At least 18 prior reports have described relative and absolute psychosocial contraindications to donation. ${ }^{408}$

\section{Disclosures and Support}

What Should Donor Candidates be told about their Likely Psychosocial Outcomes after Donation?

The psychosocial evaluation provides an opportunity to share what emotions the donor candidate might experience after donation, and the anticipated short- and long-term psychosocial outcomes after donation.

Kidney donation has a profound and multifaceted impact on the lives of many donors and influences their identity, roles and relationships. Two recent systematic reviews summarize qualitative data about donor experiences after donation. ${ }^{422,423}$ On average, donors experienced increased self-esteem, empowerment, and community awareness, but

\section{TABLE 24.}

\section{Recommendations for psychosocial factors that either exclude donation, or prevent further evaluation} until resolution

\section{Psychosocial contraindications to donation}

- A wish not to donate or marked ambivalence about donating

- Evidence of, or high suspicion of, undue pressure

- Evidence of, or high suspicion of, unreasonable or illegal secondary gain (such as an unregulated financial transaction)

- A failure to meet the requirements of informed consent (see chapter 2). This includes a donor candidate, who despite counseling, continues to have unrealistic expectations about the donation experience or potential outcomes

- Psychiatric conditions that can be treated to improve the donor candidate's predonation mental fitness and chance of a good postdonation outcome

- Past or present substance use disorder

- A psychosocial profile that predicts a level of postdonation risk that exceeds a transplant program's acceptable risk threshold. Such a profile may include an active substance use disorder, or the absence of needed psychosocial or financial support 
some also described a lack of emotional support. A subsequent systematic review of 34 prospective studies of postdonation HRQoL (1990 to 2014) found that, after mild reductions early after nephrectomy, HRQoL returned to baseline or was slightly reduced by 3 to 12 months, particularly for fatigue, but was still comparable with general population norms. ${ }^{420}$

In general, donors demonstrate good quality of life and have low rates of donation regret. That said, living donation is not without psychosocial risk, and some donors have experienced psychosocial difficulties after donation (eg, depression, anxiety, stress, worries about health), and such problems have impacted their functioning and ability to return to work.

One study was identified by the ERT that reported psychosocial outcomes in a group of donors compared with healthy nondonors, and the evidence quality was graded as very low (Evidence Report Table 8, SDC, http://links.lww.com/TP/ B434; Supplemental Appendix Table D5, SDC, http://links. lww.com/TP/B432). ${ }^{413}$ In the reviewed multicenter study, average values of 3 HRQoL scores among donors (a median of 6 years after donation) were similar to a group of nondonors and population norms. There was also no significant difference between the 2 groups in marital status, mental health visits and psychotropic medication use.

A report of 2455 living donors from 3 large US centers known as the Renal and Lung Living Donors Evaluation Study (RELIVE) cohort (93\% white race, $72 \%$ biologically related to recipient) found that HRQoL scores in living donors in the decades after donation were similar or better than the US population. ${ }^{415}$ However, 9\% of RELIVE study respondents reported one or more of the following poor psychosocial outcomes: "fair" or "poor" overall donor experience, financial burden, regret or discomfort with decision to donate, or psychological difficulties since donation. ${ }^{424}$ Recipient graft failure was the only identified predictor of reporting one or more of these poor psychosocial outcomes, and was associated with $77 \%$ higher risk (OR, $\left.{ }_{1.33} 1.77_{2.34}\right)$. Another report of 4650 privately insured living kidney donors in the United States described lower rates of depression diagnoses after donation compared with rates among age- and sexmatched beneficiaries in the same insurance system. ${ }^{414} \mathrm{~A}$ systematic review published in 2006 summarized 51 studies describing 5139 donors who were assessed on average 4 years after donation. ${ }^{425}$ Most donors who participated in followup reported no depression or anxiety, with questionnaire scores similar to the general population. The majority reported no change or an improved relationship with their recipient, spouse, family members and nonrecipient children. Some experienced an increase in self-esteem. A majority expressed no change in their attractiveness. Most donors $(96 \%)$ did not regret their donation decision.

Based on these data, we suggest that donor candidates be informed that in general, donors demonstrate good quality of life after donation. However, donor candidates should also be informed that some people experience psychosocial difficulties after donation (eg, depression, anxiety, a negative change in their relationship with the recipient, more pain than expected, a recovery time that is slower than expected, a decline in their vitality, unexpected expenses related to donation recovery, anticipated benefits that were short-lived or not met at all) or anxiety related to worries about their health (including a fear of kidney failure). ${ }^{78,425}$

\section{What Should Donors Be Told About How Donation May Impact Them Financially?}

Donor candidates and living kidney donors incur personal direct and indirect expenses as part of the evaluation and donation processes, even in countries where the donor's medical expenses are paid by the recipient's insurance or the healthcare system. Major costs include transportation, accommodation, child care, lost income (or vacation time), and missed time from work. ${ }^{426,427}$ In a prospective Canadian follow-up study, the mean cost associated with donation was $\$ 3268$ (Canadian dollars); however for $15 \%$ of donors, costs exceeded $\$ 8000 .{ }^{428}$ The expenses incurred during the predonation evaluation are also described in another recent study from the United States. ${ }^{429}$ These findings have led to recommendations that living donor candidates receive counseling about financial costs before donation, and have fostered governmental and other programs in multiple countries to reimburse donors for their legitimate expenses and attempt to maintain financial neutrality. ${ }^{430-432}$

However, there are limitations to many current cost mitigation programs. In the United States, the National Living Donor Assistance Center (NLDAC) (via a Health Resources and Services Administration grant) offers grants to offset travel and lodging costs for living donors and an accompanying person during the evaluation, donation and follow-up, but reimbursement eligibility is means tested and is based on the incomes of both the living donor candidate and recipient; further, nondirected donors have limited access because they cannot provide recipient income information. In the United States, many states have enacted legislation to offer tax deductions or credits, or other benefits, to living organ donors. ${ }^{433}$ These programs vary state by state, are underused, and have not been studied with regard to impact on financial burden of donation. Several studies have considered whether kidney donation affects one's ability to obtain life, disability and health insurance. ${ }^{434-436}$ These issues are country-specific, and should be considered in donor candidate counseling and support.

A 2016 convenience sample of informed consent forms for living donor and transplant candidate evaluation from 9 geographically diverse US programs identified misconceptions about defraying donation-related costs, reinforced by ambiguous language or omissions in the donor informed consent documents. ${ }^{437}$ Donor candidates should receive clear, accurate education on how to access available financial support as well as the limitations of available resources. In 2016, the AST launched an online financial toolkit to help donor candidates learn about the finances of being a living organ donor; this dynamic resource will be expanded and updated over time. ${ }^{438}$ Although developed from the perspective of US-based policies and resources, the information may be helpful to donor candidates in other countries. See chapter 18 for a discussion of policy related to financial support and removal of economic disincentives to donation.

\section{How Should Transplant Programs Help Donor Candidates and Donors in Need of Psychosocial Support?}

Tailored plans to support each donor candidate in having a positive psychosocial experience throughout the evaluation and donation processes should be developed, guided by donor candidate concerns and psychosocial risk factors that can be managed without excluding donation. 
Candidates ultimately excluded from living donation for any reason may experience feelings of emotional distress, disappointment and guilt, frustration from inability to control one's own body and health, and in the case of planned donation to a spouse or dependent, caregiver burden or ongoing impact of the intended recipient's illness on the donor's lifestyle. ${ }^{75}$ The transplant program should assist excluded donor candidates who have difficulty accepting their candidacy decision in receiving needed psychosocial support or psychiatric help.

In approximately $5 \%$ of living kidney donor transplants the recipient or their graft does not survive the first year. Understandably, poor recipient outcomes can be a source of stress and disappointment to the donor, and the transplant program should provide postdonation psychosocial support at such times. In some studies, but not others, poor recipient outcomes have been associated with poor psychosocial outcomes in donors. ${ }^{413,414,439-443}$ In one study, most of the adverse recipient events were beyond the first year posttransplant. ${ }^{414}$ Most donors will not experience poor short- or long-term medical or psychosocial outcomes after donation, but for those who do, the transplant program should participate to ensure that the donor receives psychosocial support or psychiatric help. The responsibility to ensure that donors receive psychological support when outcomes are poor is also described in a prior guideline. ${ }^{409}$ The Declaration of Istanbul states that all donors should be offered psychosocial services as a standard component of follow-up, ${ }^{421}$ and the CARI guideline recommends follow-up of the psychosocial impact of living kidney donation for every living donor during the first year after donation. ${ }^{409}$ Incomplete or inconsistent follow-up after the early postdonation period may lead to under-recognition of donation-related psychosocial problems in the longer-term. Models for the collection of long-term psychosocial outcomes after donation exist internationally, including the European Living Donation and Public Health (EULID) project, an 11-nation registry that uses an online tool for reporting follow-up information included psychosocial and socioeconomic outcomes. ${ }^{444}$ The national Swiss Living Donor Health Registry (SOL-DHR) follows donors lifelong and includes an 8-Item Short-Form (SF-8) and social-status questionnaire. ${ }^{445}$

\section{RESEARCH RECOMMENDATIONS}

- Better define the optimal processes and content of the psychosocial evaluation. For example, there are emerging efforts to help standardize the psychosocial evaluation through the development of semi-structured evaluation tools. ${ }^{446}$

Duerinckz et al, ${ }^{408}$ on behalf of the Ethical Legal Psychological Aspects of Transplantation Organization, summarized surveys or tools used for psychometric testing in the donor candidate evaluation from prior guidelines, consensus statements and transplant program protocols such as mood questionnaires, personality interviews and drug abuse screening. The type, number and content of tools described in prior guidelines were variable.

- The same group also recently provided a conceptual framework for the key elements in the psychosocial evaluation of living kidney donor candidates, including assessment of: i) motivation (eg, coercion, ambivalence); ii) the presence of psychopathology (eg, cognitive disturbance, mood or anxiety disorder); iii) available social resources (eg, social support, workplace support); and iv) information disclosure, understanding and risk processing (eg, elements of informed consent). ${ }^{449}$

- Determine which components of the psychosocial interview should be structured to cover relevant material while addressing the unique needs and questions of a given donor candidate.

- Perform clinical trials to compare evaluation approaches, acceptance criteria, and interventions to minimize the risk of poor postdonation psychological outcomes.

- For example, in a recent randomized trial of 113 potential kidney donors with some evidence of residual ambivalence to donation, it was suggested that motivational interviews (vs additional health education or standard care) reduced ambivalence to donation, anxiety symptoms, unexpected family-related problems, and the risk of negative physical symptoms after donation. ${ }^{417}$

\section{CHAPTER 17: ACCEPTABLE SURGICAL APPROACHES FOR DONOR NEPHRECTOMY}

Except in the case of Recommendations 17.3 and 17.7, the ERT search parameters did not identify evidence from eligible studies pertinent to the recommendations in chapter 17 and therefore the following recommendations are "Not Graded."

17.1: Renal imaging (eg, computed tomographic angiography) should be performed in all donor candidates to assess renal anatomy before nephrectomy.

17.2: The surgeon should have adequate training and experience for the surgical approach used for the donor nephrectomy.

17.3: We suggest that "mini-open" laparoscopy or hand-assisted laparoscopy by trained surgeons should be offered as optimal approaches to donor nephrectomy. However, in some circumstances, such as for donors with extensive previous surgery and/or adhesions, and at centers where laparoscopy is not routinely performed, open nephrectomy (flank or laparotomy) may be acceptable. (2D)

17.4: Robotic, single-port, and natural orifice transluminal nephrectomy should generally not be used for donor nephrectomy.

17.5: Nontransfixing clips (eg, Weck Hem-o-lok) should not be used to ligate the renal artery in donor nephrectomy; instead, renal artery transfixation by suture ligature or anchor staple within the vessel wall should be used.

17.6: In the absence of reasons to procure the right kidney (vascular, urological or other abnormalities), the left kidney should be procured in laparoscopic donor nephrectomy because of the relative technical ease associated with a longer venous pedicle.

17.7: We suggest laparoscopic procurement of the right rather than the left living donor kidney may be performed if the surgeon has adequate training and experience. $(2 D)$

17.8: Procurement of a living donor kidney with 3 or more arteries should only be undertaken by surgeons with adequate experience.

17.9: A donor candidate with atherosclerotic renal artery disease or fibromuscular dysplasia involving the orifices of both renal arteries should not donate.

\section{RATIONALE}

The choice of surgical approach and the organ for procurement (left vs right kidney) should take into consideration the experience of the surgeon as well as any renal functional, parenchymal, vascular or urological abnormalities. Relevant outcomes to consider in comparing surgical approaches 
include: mortality; perioperative complications (eg, bleeding, reoperation, incisional hernias); quality of life, including pain and wound cosmesis; recovery time; and graft outcomes in the recipient. Studies often report operative time as a surrogate measure for risks of anesthesia-related complications. The ERT identified 4 recently published systematic reviews describing perioperative outcomes after living donor nephrectomy. ${ }^{448-450}$

\section{Laparoscopic versus Open Nephrectomy}

The ERT identified 3 systematic reviews comparing outcomes after laparoscopic versus open nephrectomy $448,450,451$ (Evidence Report Table 3, SDC, http://links.lww.com/TP/ B434; Supplemental Appendix Table C3, SDC, http://links. lww.com/TP/B432). Collectively, these reviews provide very low to high quality evidence for several outcomes, including operative time, blood loss, warm ischemia time, reoperation, length of hospital stay, and return to work. Overall, laparoscopic and open donor nephrectomies have comparable outcomes with regard to donor safety and graft function. Simple open (flank subcostal retroperitoneal) donor nephrectomy is generally performed with shorter operative times than laparoscopic nephrectomy but may incur slightly higher blood loss. Open nephrectomy is no longer considered the standard of care due to a longer incision and generally longer associated hospital stay, higher analgesia requirements, and longer convalescence period. By 2013, almost all living donor nephrectomies in the United States were performed laparoscopically, and, with increasing experience, few are converted to open procedures. ${ }^{94}$ However, in unusual circumstances, such as for donors with extensive previous surgery and/or adhesions, and at centers where laparoscopy is not routine, open nephrectomy (flank or laparotomy) may be justified. Of note, as for most surgical procedures, experience is likely to be an important determinant of outcomes, and trials and observational series were generally performed at experienced centers by limited numbers of surgeons.

\section{Operative Time}

Fonouni et $\mathrm{al}^{448}$ reported a trend towards longer average operative time for laparoscopic compared with open donor nephrectomy, and Yuan et al and Wilson et al confirmed longer operative times for laparoscopic procedures. ${ }^{450,451}$ Metaanalysis by Yuan et $\mathrm{al}^{451}$ estimated that laparoscopic nephrectomy requires an average of 51 minutes longer surgical time (95\% CI, 33-68 minutes). Surgery times averaged 2 to 3 hours for open nephrectomy and 3 to 4 hours for laparoscopic nephrectomy.

\section{Blood Loss}

Five of the 8 publications included in the review by Fonouni et $\mathrm{al}^{448}$ reported less perioperative blood loss with laparoscopic nephrectomy, while the other 3 found no significant differences in blood loss between the approaches. Metaanalysis by Yuan et al, ${ }^{451}$ including 12 studies with 917 donors, found a small but statistically significantly increase in perioperative blood loss with open vs laparoscopic nephrectomy (mean difference, $-99.6 \mathrm{~mL}$; 95\% CI, -165.9 to $-33.4)$ or open versus hand-assisted laparoscopy $(-112.8 \mathrm{~mL}$; $95 \% \mathrm{CI},-169.1$ to -56.0$).{ }^{451}$

\section{Warm Ischemia Time}

Data regarding warm ischemia times with laparoscopic versus open nephrectomy are conflicting. In 9 of the 11 publications evaluated by Fonouni et al, ${ }^{448}$ longer warm ischemia times were reported in the laparoscopic nephrectomies compared with open nephrectomies. Similarly, all included studies in the review by Wilson et $\mathrm{a}^{450}$ found longer warm ischemia times in the laparoscopic groups, with differences ranging from 2 to 17 minutes. In contrast, based on data from 15 trials including data for 1598 donors, Yuan et al identified a small, significant decrease in warm ischemia time with laparoscopic compared with open nephrectomy (mean difference, -1.8 minutes; 95\% CI, 1.3-2.2); warm ischemia time also trended lower for hand-assisted laparoscopy compared with open nephrectomy (mean difference, 0.4 minutes; $95 \%$ CI, -0.2 to 1.0 ), but this difference was not statistically significant. Overall, there were no clinically important differences in warm ischemia times with laparoscopic versus open donor nephrectomies. Wilson et $\mathrm{al}^{450}$ found no significant differences between laparoscopic and open nephrectomy for early graft loss (RR, $0.060 .31_{1.48} ;$ ), delayed graft function ( $\left.R R, 0.521 .09_{2.30}\right)$, acute rejection (RR, $0.871 .41_{2.27}$ ), ureteric complications (RR, $0.691 .51_{3.31} ;$ ), donor kidney function at 1 year (standardized mean difference, $0.15 ; 95 \% \mathrm{CI},-0.11$ to 0.41 ) or graft loss at 1 year $\left(\mathrm{RR}, 0.150 .76_{3.85}\right)$.

\section{Reoperation}

Fonouni et $\mathrm{al}^{448}$ included 7 publications (both trials and meta-analyses with unreported donor sample sizes) reporting reoperation rates, and found mixed results. Although 4 of the publications found no difference between the groups, 3 found higher reoperation rates in the laparoscopic compared with the open group. Based on data from 6 studies, Wilson et $\mathrm{al}^{450}$ found fewer reoperations with open nephrectomy compared with laparoscopic nephrectomy, but this difference was not statistically different ( $\left.R R,{ }_{0.09} 0.57_{3.64}\right)$.

\section{Length of Hospital Stay}

Among 9 publications (with unreported donor sample sizes) reviewed by Fonouni et al, ${ }^{448} 4$ of the 9 found no difference in length of hospital stay between the groups, while the remaining 5 found longer length of stay with open versus laparoscopic nephrectomy. Meta-analysis of 16 studies (including 1681 donors) by Yuan et al ${ }^{451}$ identified significantly shorter length of hospital stay after laparoscopic compared with open nephrectomy (mean difference, -1.3 days; $95 \%$ CI, -1.7 to -0.8$).{ }^{451}$ Significant trends toward shorter length of stay were found with standard laparoscopic (mean difference, -1.3 days; $95 \% \mathrm{CI},-1.9$ to -0.7 ) and hand-assisted laparoscopic (mean difference, -1.3 days; $95 \% \mathrm{CI},-2.1$ to -0.5 ) compared with open nephrectomy. ${ }^{450}$

\section{Return to Work}

The time to return to work was reported in one systematic review based on pooled data from 9 studies capturing 1016 donors. Laparoscopic nephrectomy was associated with a statistically significant and clinically meaningful reduction in time to return to work (mean difference, -16.4 days; $95 \%$ CI, -23.0 to -9.7$).{ }^{451}$ 


\section{Pain and Physical Functioning}

Among 6 studies describing analgesia requirements in the review by Wilson et al, ${ }^{450} 4$ reported significant reductions in morphine requirements with laparoscopic versus open neprhrectomy. One study showed that donors who underwent laparoscopic nephrectomy required fewer days of analgesia postdischarge (3.3 vs 7.8 days, $P<0.001$ ), while another reported a nonsignificant reduction in visual analogue pain scale scores after laparoscopic nephrectomy. The meta-analysis by Yuan et $\mathrm{al}^{451}$ of 3 studies including 306 donors showed superior 36-item Medical Outcomes Study Short Form (SF-36) physical functioning (mean difference, 6.6; 95\% CI, 2.3$10.8 ; P=0.002$ ) and bodily pain scores (mean difference, 5.9; 95\% CI, 1.6-10.3; $P=0.007$ ) with laparoscopic compared with open nephrectomy.

\section{What Prior Guidelines Recommend}

Other guidelines have also recommended laparoscopic or minimally surgical invasive approaches for living donor nephrectomy ${ }^{48,50,54,452}$ based on evidence supporting lower morbidity, less pain and analgesia requirements, and earlier return to normal activity compared with open nephrectomy. CARI notes equivalent rates of major complications with laparoscopic versus open nephrectomy (with complications after laparoscopy largely due to catastrophic bleeding due to failure of intraoperative securing of the vascular pedicle), larger resource requirements for laparoscopy, but advantages with laparoscopy in terms of reduced analgesia requirements and faster return to normal activity. ${ }^{453}$ The European Association of Urology concludes that laparoscopy offers equivalent complication rates and graft outcomes as open nephrectomy but shorter convalescence and better cosmetic results. ${ }^{55}$ The Japanese Society of Endourology emphasizes the importance of experience, recommending that institutions commencing laparoscopic living donor nephrectomy should do so under the direction of an accredited laparoscopist. ${ }^{452}$

\section{Minimally Invasive Nephrectomy Approaches: Standard Laparoscopic Versus Hand-Assisted Laparoscopic Nephrectomy}

Three systematic reviews presented evidence comparing standard living donor laparoscopic nephrectomy and handassisted laparoscopic living donor nephrectomy, and summarize very low to low quality evidence supporting similar outcomes across these approaches ${ }^{48,450,451}$ (Evidence Report Table 4, SDC, http://links.lww.com/TP/B434; Supplemental Appendix Table C4, SDC, http://links.lww.com/TP/B432).

\section{Operative Time}

Yuan et $\mathrm{al}^{451}$ reviewed 7 trials including 387 donors that compared hand-assisted laparoscopic nephrectomy to standard laparoscopic nephrectomy, and found no statistical difference in operating time by meta-analysis (mean difference, -24.6 minutes; $95 \%$ CI, -50.8 to 1.7 ).

\section{Blood Loss}

Wilson et $\mathrm{al}^{450}$ reported varying results for blood loss across the studies of standard laparoscopic and hand-assisted nephrectomy included in their review, but overall found no significant differences between the groups. Similarly, Yuan et $\mathrm{al}^{451}$ found no significant differences in blood loss with standard compared with hand-assisted laparoscopic nephrectomy (mean difference, $-20.7 \mathrm{~mL}$; 95\% CI, -43.9 to 2.6).

\section{Warm Ischemia Time}

Yuan et $\mathrm{al}^{451}$ found a small, statistically significant reduction in warm ischemia time with hand-assisted compared with standard laparoscopy (mean difference, -1.0 minutes; $95 \%$ CI, -1.4 to -0.6 ), but this small difference does not appear to be clinically meaningful.

\section{Length of Hospital Stay}

Based on 6 studies comprising 320 donors, donors undergoing standard laparoscopic nephrectomy had a slight reduction in average length hospital stay compared to those undergoing hand-assisted laparoscopic nephrectomy (mean difference, 0.3 days; $95 \%$ CI, 0.1-0.6) ${ }^{451}$ although the difference (less than 1 day) appears to be modest.

A clinical trial at 2 centers published after these systematic reviews described 190 donors randomized to hand-assisted or standard laparoscopy for left donor nephrectomy. ${ }^{454}$ Hand-assisted laparoscopy resulted in shorter operative time (mean, 159 vs 188 minutes; $P=0.001$ ) and a small reduction in warm ischemia time ( 2 vs 5 minutes; $P<0.001)$. Intraoperative complication rate $(5 \%$ vs $11 \%, P=0.12)$, length of stay (both 3 days; $P=0.14$ ), postoperative complication rate $(8 \%$ vs $8 \% ; P=1.00)$, potential graft-related complications $(6 \%$ vs $13 \% ; P=0.14)$, and physical function at 1 month follow-up $(P=0.55)$ did not significantly differ between the groups.

\section{Novel Minimally-Invasive Nephrectomy Approaches}

In recent years, innovations in laparoscopic surgery have provided transplant surgeons with a range of techniques and minimally invasive instruments. Robotic-assisted, total robotic, and single port nephrectomies have been described in case reports, small series, and small trials at experienced centers. ${ }^{455-459}$

A systematic review and meta-analysis published after the ERT's systematic review for this guideline examined $190 \mathrm{ar}-$ ticles published through March 2016 reporting short-term complications after minimally invasive donor nephrectomy ${ }^{460}$; of these, outcomes from 41 articles that compared 2 or more minimally invasive nephrectomy approaches were included in a meta-analysis. Publication dates of identified articles ranged from 2000 to 2015; most studies were retrospective case series. Although the sample included some prospective series, there were only 16 RCTs. The distribution of 32038 donors according to operative technique was: laparoscopic, 57.4\%; hand-assisted laparoscopic, 25.3\%; miniopen, 4.5\%; hand-assisted retroperitoneoscopic, 3.8\%; retroperitoneoscopic, $3.7 \%$; single-port laparoscopic (ie, laparoscopic single-site surgery [LESS]), $3.8 \%$; and roboticassisted laparoscopic, $1.3 \%$. Overall, conversion rate to traditional open nephrectomy was $1.1 \%$. Intraoperative complication rate was $2.3 \%$, most commonly bleeding $(1.5 \%)$. Postoperative complications occurred in $7.3 \%$ of donors, including infectious complications in $2.6 \%$ (mainly wound infections, $1.6 \%$ ) and bleeding in $1.0 \%$. Reported mortality rate was $0.01 \%$.

Among 7 studies (2 RCTs, 2 prospective, and 3 retrospective) comparing laparoscopic $(n=1159)$ with retroperitoneoscopic 
$(\mathrm{n}=311)$ techniques, there were no significant differences in rates of conversion, overall intraoperative or perioperative complications. ${ }^{460}$ Based on 8 studies (3 RCTs, 5 prospective) comparing mini-open $(\mathrm{n}=322)$ to endoscopic $(\mathrm{n}=288)$ techniques (ie, laparoperitoneoscopic, retroperitoneoscopic, with or without hand assistance), intraoperative complications were more frequently seen in endoscopic procedures $(8.2 \%$ vs $3.4 \%$; RR $\left.{ }_{1.13} 2.45_{5.35} ; P=0.02\right)$. This difference was mostly due to intraoperative organ damage, which demonstrated a trend in favor of open procedures $(0 \%$ vs $2.8 \%$; $\mathrm{RR}_{0.91} 5.18_{29.35} ; P=0.06$ ). The postoperative complication rate was comparable after mini-open versus laparoscopic donor nephrectomy. Ten studies (3 RCTs, 1 prospective study, 6 retrospective series) compared single-port $(\mathrm{n}=764)$ with multiport laparoscopic $(\mathrm{n}=1214)$ procedures, among which pulmonary complications were more frequently reported after LESS donor nephrectomy (1.5\% vs $0 \%$; RR $1.257 .51_{44.94}$; $P=0.03)$. The slightly higher incidence of postoperative pain after LESS compared with multiport nephrectomy was not statistically significant $\left(2.7 \%\right.$ vs $0.8 \%$; $\mathrm{RR}_{0.90} 3.56_{14.11}$; $P=0.07)$.

Limitations of this systematic review ${ }^{460}$ include the varying study designs, outcome definitions and ascertainment methodologies. A Cochrane review of RCTs published through January 2016 comparing LESS donor nephrectomy with laparoscopic donor nephrectomy identified 3 studies including 179 patients, and concluded that data were insufficient for drawing inferences on outcomes. ${ }^{461}$ At the present time, given the current limited experience and lack of robust safety and outcomes data, robotic, single-port, and natural orifice transluminal nephrectomies are not currently standard of care approaches to living donor nephrectomy and should only be performed by surgeons with adequate training and experience, and after informed consent.

\section{What Prior Guidelines Recommend}

The ERBP also recommends that the choice between minimal invasive and standard laparoscopic procedure should be based on the local expertise. ${ }^{50}$ No other guidelines recommend a preference for standard laparoscopy versus handassisted or other minimally invasive approaches.

\section{Left Versus Right Laparoscopic Nephrectomy}

In the absence of reasons to procure the right kidney (eg, vascular or urological abnormalities), the left kidney should be procured in laparoscopic living donor nephrectomy because of the relative technical ease associated with a longer venous pedicle. However, the choice of which kidney to procure should be made to minimize short-term and long-term complications in the donor. Thus, the presence of renal parenchymal, vascular or urological abnormalities which are not contraindications to donation may favor the use of an abnormal right kidney for donation and transplantation, for the goal of leaving the donor with the "more normal" kidney and/or to minimize complications related to multiple vascular pedicles. Recent evidence supports safe laparoscopic procurement of the right kidney without increased risk of adverse outcomes compared with procurement of the left kidney, although the evidence is weak. The ERT examined one systematic review by Liu et $\mathrm{a}^{449}$ comparing right with left laparoscopic living donor nephrectomy, and identified very low quality evidence for similar operative times, blood loss, warm ischemia times, and length of stay at experienced centers (Evidence Report Table 5, SDC, http://links.lww.com/TP/B434; Supplemental Appendix Table C5, SDC, http://links.lww. $\mathrm{com} / \mathrm{TP} / \mathrm{B} 432$ ). Although complication rates of minimally invasive surgery are generally low with experienced surgeons, complications can be life threatening, and therefore proper training and experience is critical.

\section{Operative Time}

Among 14 studies including 2656 donors with available data, operative times were similar with left and right laparoscopic nephrectomy (weighted mean difference, 1.4 minutes; $95 \% \mathrm{CI},-11.7$ to 14.4$).{ }^{449}$

\section{Blood Loss}

Based on data from 15 studies including 3033 donors, blood loss was similar between donors who underwent left versus right nephrectomy (weighted mean difference, $4.4 \mathrm{~mL}$; $95 \%$ CI, -19.8 to 28.6$).{ }^{449}$

\section{Warm Ischemia Time}

Among 18 studies including 3516 donors, warm ischemia time was also similar among donors undergoing left versus right nephrectomy (weighted mean difference, -0.02 minutes; $95 \%$ CI, -0.4 to 0.05$).{ }^{449}$

\section{Length of Hospital Stay}

The mean length of hospital stay in 11 studies including 1370 donors was similar between those undergoing left versus right nephrectomy (weighted mean difference, 0.05 days; $95 \%$ CI, -0.08 to 0.02 ). ${ }^{449}$

A subsequent study of 58599 living donor nephrectomies from a US registry, including $13.9 \%$ right nephrectomies, found statistically significant but numerically small differences in outcomes after right versus left donor nephrectomy, including higher rates of conversion from laparoscopic to open nephrectomy $(1.9 \%$ vs $0.95 \%, P \leq 0.00001$; OR $\left.1.162 .02_{2.52}\right)$, delayed allograft function $(5.7 \%$ vs $4.2 \%$, $P<0.0001$; HR $\left.1.241 .38_{1.53}\right)$ and early graft thrombosis $\left(1.1 \%\right.$ vs $\left.0.79 \%, P=0.006 ; \mathrm{HR}_{1.18} 1.481 .86\right) .{ }^{462}$ The authors concluded that the laterality of donor nephrectomy should be based on surgeon preference and experience.

\section{Laparoscopic Procedure Type for Right Nephrectomy}

Use of hand-assisted laparoscopy over standard laparoscopy for right donor nephrectomy is controversial, as some authors report caudal position of the liver that limits the working space for the hand assisted approach. ${ }^{463} \mathrm{~A}$ trial at a referral center with longstanding expertise in the standard laparoscopic right nephrectomy explored the safety and feasibility of right hand-assisted approach by randomly assigning 40 donors to either approach. ${ }^{464}$ As compared with laparoscopic right nephrectomy, hand-assisted right nephrectomy resulted in slightly shorter warm ischemia time ( 2.8 vs 3.9 minutes; $P<0.001)$ and increased blood loss ( 187 vs $50 \mathrm{~mL}, P<0.001$ ), while operative time, complication rate, pain, hospital stay and 1 year quality of life scores were not significantly different between the groups. 


\section{Multiple Renal Arteries}

Kidneys with multiple renal arteries are common. A study of CT scan results performed during living donor evaluation reported a prevalence of $18 \%$ to $30 \%$ for multiple renal arteries, with up to $15 \%$ bilateral involvement. ${ }^{465-467}$ Multiple renal arteries may adversely affect donor safety and recipient outcome. Multiple renal arteries may lead to intraoperative technical difficulties and complications, such as increased operative time, complicated dissection, or bleeding. Furthermore, either arterial reconstructions need to be created after procurement or multiple arterial anastomoses are needed in the recipient, both of which may be associated with complications. A small accessory artery that supplies a minor part of the upper pole (subjectively assessed using predonation CT scans) can often be safely sacrificed. However, an accessory artery that vascularizes the lower pole and the proximal ureter must be saved and reconstructed after nephrectomy. In the case of a complex venous anatomy, there is usually only one domain vein, and the remaining veins can be tied off. A systemic review by Ahmadi et al $^{468}$ capturing reports of outcomes associated with vascular multiplicity in living donors in the past decade concluded that renal arterial multiplicity (up to 3 renal arteries) and venous anomalies should not be a contraindication to living kidney donation. Vascular anomalies require longer operative times to manage, but were not associated with significant negative impact on donor or graft outcomes with modern surgical techniques and high surgical skills. As most living donors with multiple renal arteries in the reported studies had a maximum of 3 renal arteries, conclusions cannot be drawn for donors with 4 or more arteries. Prior guidelines have also concluded that multiple arteries is generally not a contraindication to living kidney donation. ${ }^{48,54,55}$

\section{Nontransfixing Vascular Clips}

Hemorrhagic deaths of living kidney donors from failure of nontransfixing vascular clips (eg, Weck Hem-o-lok; Teleflex Medical, Bannockburn, IL) used to ligate the donor renal artery were first documented in 2006..$^{469,470}$ Despite a Class II recall of the Hem-o-lok clip for laparoscopic donor nephrectomy by the US FDA in 2006, and a FDA Black Box Warning that use of the Weck Hem-o-lok hemostatic clip to ligate the renal artery in living donor nephrectomy is contraindicated, use of these clips is still reported. A 2011 ASTS survey reported that Hem-o-lok or other clips are still used by some surgeons as sole means of arterial control in laparoscopic donor nephrectomy. ${ }^{469}$ Subsequently, in a 2013 survey of 645 members of the European Society for Organ Transplantation, $20 \%$ of respondents reported use of clips (locking and nonlocking) to close the arterial stump. ${ }^{471}$ Of the 121 reported hemorrhagic events, slippage and dislodgement of clips occurred in at least $58(47.9 \%) .{ }^{471}$ All surgeons operating on a living organ donor should select vascular control techniques that entail tissue transfixion and assure a safe operative recovery. The Hem-o-lok and other surgical clips should not be used to ligate the donor renal artery.

\section{What Prior Guidelines Recommend}

The CARI guideline states that the use of a nontransfixing technique for securing the renal artery is not recommended, particularly with laparoscopic donor nephrectomy. ${ }^{453}$ In the current guideline, we recommend that nontransfixing clips should never be used to ligate the renal artery in donor nephrectomy.

\section{RESEARCH RECOMMENDATIONS}

- Define the incidence of perioperative outcomes in representative donor samples (ie, not limited to experienced centers with a limited number of surgeons) according to nephrectomy surgical approach and side.

- Define outcomes of nephrectomy surgical approaches according to baseline donor characteristics (eg, BMI, renal vascular anatomy).

- Define the outcomes of novel minimally invasive approaches to donor nephrectomy to guide future recommendations and appropriate use of these techniques.

- Determine the optimal training and experience levels necessary to define proficiency with donor nephrectomy approaches. ${ }^{472}$

\section{CHAPTER 18: ETHICAL, LEGAL AND POLICY CONSIDERATIONS}

The ERT search parameters did not identify evidence from eligible studies pertinent to the recommendations in chapter 18 and therefore the following recommendations are "Not Graded.”

\section{Ethical and Legal Framework}

18.1: Local laws and regulations on living donation should be followed and explained as needed to donor candidates.

18.2: Where local laws or policies impede the ethical practice of living donation, avenues to advocate for change should be explored.

18.3: Autonomy (self-determination) in the willingness or not to be considered as a living donor should be respected during all phases of the evaluation and donation processes. Transplant programs should support autonomy through a fully informed consent process.

\section{Policies for Donor Candidate Identification}

18.4: Public awareness of opportunities for living donation should be increased through education, donor advocacy, evaluation efficiencies, and removal of disincentives.

18.5: Transplant candidates should be assisted in identifying living donor candidates, as long as these efforts respect donor autonomy and do not exert undue pressure to donate.

18.6: Donor candidates should be informed of the dangers of transplant tourism.

18.7: Transplant programs should define and disclose their policies for the acceptance of donor candidates identified through public solicitation.

\section{Financial Support}

18.8: Donor candidates should be informed of the availability of legitimate financial assistance for expenses from evaluation and donation.

\section{Communication of Policies}

18.9: Nondirected donors and donors participating in paired donation should be informed of the transplant program's policy on contact with the recipient and other paired donation participants at all stages in the donation process. 
18.10: Transplant programs should disclose the extent of the expected postdonation program-patient relationship before donation, including whether the donor can seek medical care at the transplant center after donation.

18.11: Regional policies should ensure access to kidney replacement therapy (dialysis and/or transplantation) for donors who develop kidney failure.

\section{RATIONALE}

\section{Ethical and Legal Framework for Living Donation}

Living kidney donation must be practiced within a framework of the laws and regulations of each country and its governing or regulatory bodies. The legal framework gives legitimacy to living donation and provides some protection to the donor. ${ }^{473}$ All practitioners in transplant programs should be aware of relevant laws and regulations that pertain to the living donor transplant program. ${ }^{474}$ Ethical tenets and specific transplant program processes are applied to minimize donor risk. See chapter 1 for discussion of the conceptual framework for decision-making related to donor acceptance, and roles and responsibilities in donationrelated care, and chapter 2 for details on the processes and content of informed consent.

When there is opportunity to identify needs for policy and legal changes to improve the practice of living donation, transplant programs should actively participate in the review and process to update or change laws, regulations and policies as needed. ${ }^{475}$ Laws to regulate donation include, but are not limited to, those that: a) protect the vulnerable (eg, the cognitively impaired, young people, dependent persons) ${ }^{53,476,477}$; b) respect autonomy (eg, require informed consent); c) prohibit incentives to donate that may create undue influence (eg, payment for organs in money or in kind). ${ }^{430}$ Other relevant laws and policies related to donor protections include insurability criteria related to donation status, state tax credits for donation-related expenses, and access to medical leave after donation.

Transplant professionals have an ethical duty to act in the best interests of donor candidates and donors. Selfdetermination related to the donation decision reflects a view of people as autonomous agents. ${ }^{478}$ Donor autonomy in the willingness to be considered as a donor candidate or to withdraw should be respected during all phases of the evaluation and donation process. Programs should support autonomy through a fully informed consent process (chapter 2). Balancing risks and benefits to the donor as well as respecting the donor's wishes for donation addresses the principle of beneficence.

In the United States, concern that kidney paired donation could be construed as violating the prohibition of NOTA to transfers of organs for valuable consideration was addressed with a specific amendment ("Norwood Act" of 2007) that explicitly defined paired donation as exempt from NOTA violation. ${ }^{479}$ Although nonsimultaneous chains initiated by nondirected donors do not technically fall within the exemption for kidney paired donation, there is arguably no need for an exemption with respect to chains initiated by nondirected donors because they are designed as a series of gifts, rather than as an exchange of valuable consideration. See chapter 3 for discussion of clinical and educational issues related to paired donation.

\section{Policies for Donor Candidate Identification}

People may not be aware of or have accurate knowledge of living donation. ${ }^{480}$ Recipient candidates may not feel comfortable sharing their need for an organ donor with their social network for reasons including not knowing how to ask, misunderstanding of risks, and fear for harm to the donor candidate. ${ }^{481,482}$ A 2015 AST Live Donor Community of Practice consensus statement on "Best Practices in Live Kidney Donation" deemed implementation of approaches to empower transplant candidates and their loved one in their search for a living donor to be a high priority. ${ }^{148,483}$ Developing effective methods to address the growing organ shortage, including through living donor transplantation, is the highest stated priority in the 2015 US OPTN Strategic Plan ${ }^{484}$ and was endorsed in a June 2016 White House Summit on Organ Donation. ${ }^{485}$ Appropriate strategies for promoting living donation might include education (public, clinic-based, home-based), use of advocated (eg, "live donor champions"), efficiencies in the evaluation of donor candidates (eg, use of new information technology) and the removal of disincentives. ${ }^{486-490}$ Effective interventions may include participation of family and friends of the transplant candidate to increase knowledge and awareness of living donation within the patient's social network. The evidence supporting use of social media tools to enable transplant candidates to share their need for a living donor with their social network is at an early stage, ${ }^{491}$ but given the growing use of the internet and devices such as smartphones globally, application of technology to donor identification is anticipated to grow and was referenced by the White House Office of Science and Technology Policy. ${ }^{492}$

The ethical appropriateness of strategies for promoting living donation may vary with the entity employing the strategy. ${ }^{493}$ For example, direct promotion of donation by transplant programs may raise ethical concerns for conflict of interest and risk eroding public trust and the quality of donor decision-making, but training and support of advocated (eg, "live donor champions") can be an appropriate alternative if employed conscientiously, including the careful use of anticoercion training. Nonprofit groups separate from transplant programs may have more freedom in promoting donation without challenging ethical norms underlying the physician-patient relationship, but still bear ethical responsibilities to present evidence-based information and maintain public trust. Development of "best practices" related to use of well-designed, evidence-based approaches including technology to efficiently and ethically expand opportunities for willing, well-informed patients and potential donors to pursue living donor transplantation and living donation is an important priority. All strategies for living donor identification must respect donor autonomy and cannot exert undue pressure to donate. Critically, a wish not to donate must always be respected. ${ }^{494}$

Some people respond to public solicitations by offering to be a kidney donor. ${ }^{495}$ By evaluating these donor candidates, transplant programs are enabling donation. ${ }^{496}$ In this setting, the standard evaluation needs to address additional issues related to the parties' limited knowledge of each other, and the potential for exploitation. ${ }^{497}$ Recently, the Canadian Society of Transplantation issued a position paper that addressed issues such as privacy, informed consent, and donor follow-up associated with public donor solicitation. ${ }^{84}$ 


\section{Financial Support for Living Donors and Removal of Economic Disincentives}

Financial support for living kidney donors can be divided into cost replacement and financial gain. Living donor candidates have the right to be informed of available legitimate cost-replacements programs for evaluation and donationrelated expenses. ${ }^{430,432}$ Initiatives to remove financial disincentives to kidney donation (ie, replacement of costs incurred during the evaluation and donation such as lost income, travel costs, accommodation costs; avoidance of insurance discrimination) are acceptable as an issue of justice. ${ }^{430} \mathrm{Mul}-$ tiple prior living donation guidelines and viewpoints endorse reimbursement for out-of-pocket expenses incurred during the evaluation and donation processes, independent of the final decision to proceed with donation. ${ }^{47-49,498-500}$

In the United States, the prohibition of organ exchange for "valuable consideration" in NOTA has been clarified as not prohibiting "reasonable payments associated with... the expenses of travel, housing, and lost wages incurred by the donor of a human organ in connection with the donation of the organ." ${ }^{501}$ To date, developing programs for wage reimbursement has posed the most challenges internationally, including the need for accepted definition of lost wages and the potentially large cost of reimbursing very high-income donors. ${ }^{502}$ In December 2016, New Zealand passed legislation to provide $100 \%$ of a donor's earnings for up to 12 weeks after donation plus childcare assistance for donors who need it while they recover. ${ }^{503}$ Also in December 2016, the US NLDAC, which to date has provided funds to reimburse donation-related travel and lodging costs, announced that it will conduct an RCT to assess the impact of interventions intended to remove financial barriers to living organ donation through wage reimbursement. ${ }^{504}$ Recommendations to offer life and disability and/or health insurance for living donors, or to pass legislation to offer tax credits and ensure employment and insurability protections to donors, have also been advanced. ${ }^{55,432,500,505-508}$

In 2016, the AST launched an online financial toolkit to help donor candidates learn about the finances of being a living organ donor; this dynamic resource will be expanded and updated over time. ${ }^{438}$ Although developed from the perspective of United States-based policies and resources, the information may be helpful to donor candidates in other countries. See chapter 16 for a discussion of the potential financial impact of living donation.

\section{Financial Incentives for Donation}

Payment for a kidney is illegal in almost all parts of the world. Suggestions to provide financial incentives for donation elicit some fears that vulnerable people such as the poor will be exploited, and that public views of donation and transplantation will be damaged. ${ }^{509}$ The Declaration of Istanbul asserts that because transplant commercialism targets impoverished and otherwise vulnerable donors, it leads inexorably to inequity and injustice and should be prohibited. ${ }^{421}$ However, the debate on the acceptability of incentives continues and a formal examination of donor incentives within clinical trials has been proposed. ${ }^{509,510}$

\section{Communication of Policies and Postdonation Follow-Up Care}

Living anonymous donors are also referred to as nondirected living donors. ${ }^{411}$ These are living donors who offer a kidney to a transplant center to allocate, as they do not have an identified recipient. Nondirected donors may also donate by participating in a KPD program or as part of a series of matched donor and recipient pairs allowing for a chain of multiple transplants. Some nondirected living donors and their recipients may request contact with each other postdonation. Clarification before donation of the center's policy on such contacts enables participants to adjust their expectations after donation. ${ }^{511}$

The extent of the physician-patient relationship after donation should be communicated before donation, including whether the donor can seek medical care at the transplant center after the donation. See chapter 19 for discussion of postdonation follow-up recommendations.

Kidney failure is a rare event after living kidney donation, but fatal in the absence of kidney replacement therapy. Federally funded programs to assure access to dialysis and transplantation can be promoted as a strategy to protect the safety of living donors if the remaining kidney fails after donation. Payment for dialysis services, payment for living or deceased donor transplantation, and allocation priority for deceased donor kidney transplantation are examples of such programs. Many countries provide substantial assistance for dialysis and transplantation as part of public health services. For example in the United States, prior living donors receive additional priority points for deceased donor kidney transplants, ${ }^{512}$ which has been associated with higher transplant rates, shorter time to transplant, and receipt of higher quality allografts compared to candidates with otherwise similar clinical profiles. ${ }^{513-515}$ However, the transplant center must report prior donor status for the patient to receive priority. ${ }^{516}$ Priority for prior living donors can also be incorporated in KPD matching algorithms. ${ }^{61}$ In Israel, when a living donor develops ESKD subsequent to the donation, the living donor can designate a family member to receive priority on the deceased donor transplant waitlist. ${ }^{517}$ The purpose is to reduce the disincentive to current donation based on concern that a family member may need a kidney transplant in the future. The impact of this priority on the national allocation system and on attitudes and concerns about living donation warrants further study. ${ }^{518}$

\section{RESEARCH RECOMMENDATIONS}

- Explore approaches to and impact of strengthening partnerships between general nephrologists, dialysis providers, and transplant centers regarding living donor kidney transplant education, access and disparities. ${ }^{483}$

- Examine the impact of patient and public education on increasing donation from living donors. ${ }^{483,490,519}$

- Develop "best practices" related to use of well-designed, evidence-based approaches to efficiently and ethically expand opportunities for willing, well-informed patients and potential donors to pursue living donor transplantation and living donation. ${ }^{492,496,497}$ Research on strategies to promote living donation should evaluate not only efficacy in increasing donation but also effects on attitudes, quality of donor decisionmaking, and informed consent. ${ }^{493}$

- Examine the experiences of individuals exposed to different forms of "nonargumentative" influences promoting living donation (ie, approaches to shaping behavior that do not attempt to persuade through reason, such as appeals to emotion, messenger effects, and social norms) and whether exposed individuals feel the influence was appropriate. ${ }^{493}$ 
- Examine strategies for reducing financial barriers to living donation, with particular attention to impact on current disparities in living donor kidney transplantation. ${ }^{432,502}$

- Determine optimal approaches to allowing or restricting contact between nondirected kidney donors and their recipients. ${ }^{511}$

- Evaluate the outcomes of incentivizing organ donation via ethically and legally acceptable methods. ${ }^{520,521}$

\section{CHAPTER 19: POSTDONATION FOLLOW-UP CARE}

The ERT search parameters did not identify evidence from eligible studies pertinent to the recommendations in chapter 19 and therefore the following recommendations are "Not Graded."

19.1: A personalized postdonation care plan should be provided before donation to clearly describe follow-up care recommendations, who will provide the care, and how often.

19.2: The following should be performed at least annually postdonation:

- Blood pressure measurement

- BMI measurement

- Serum creatinine measurement with GFR estimation

- Albuminuria measurement

- Review and promotion of a healthy lifestyle including regular exercise, healthy diet and abstinence from tobacco

- Review and support of psychosocial health and wellbeing

19.3: Donors should be monitored for CKD, and those meeting criteria for CKD should be managed according to the 2012 KDIGO CKD Guideline.

19.4: Donors should receive age-appropriate healthcare maintenance, and management of clinical conditions and health risk factors according to clinical practice guidelines for the regional population.

\section{RATIONALE}

\section{Rationale for Postdonation Follow-Up}

The living donor evaluation should be regarded as the initial stages of a long-term, collaborative relationship between 2 parties, the donor candidate and the transplant program. ${ }^{196}$ The donor enters the relationship with an interest in offering an altruistic and life-extending gift of an organ for transplantation if he or she is healthy enough to donate and meets the transplant center's acceptance criteria. Grounded in primary concern for the well-being of the donor, the transplant center should promote the donor's autonomy, safety and long-term health throughout all phases of care including evaluation, donation surgery, long-term follow-up and donation-related care. Early postdonation follow-up care is routinely practiced as part of postoperative care. In contrast, responsibility for the coordination and performance of longterm donor follow-up has raised controversies regarding financial and time burden on both transplant centers and donors, especially in countries without universal health insurance. ${ }^{522}$ Monitoring incurs costs and may provide only limited information for the majority of donors who may demonstrate stable clinical status and well-being. However, as articulated in a 2011 consensus conference report, ${ }^{523}$ these concerns are outweighed by fundamental ethical principles and clinical needs to support the practice of living donation, including:

- The need to provide accurate outcomes information to donor candidates and their recipients as a basis for informed consent, especially regarding trends in outcomes and incremental hazards that may be associated with race/ethnicity, baseline comorbidity, changes in surgical approaches and management strategies.

- The need to acquire more robust outcomes data to improve the evaluation process and to provide reliable counseling for donor candidates tailored for demographic and health profile.

- The possibility of identification of individual donor clinical problems through surveillance at a time when intervention is possible.

- Provision of program-specific feedback to guide quality assurance and performance improvement.

- Recognition of the professional obligation of the transplant community to continue to collect and monitor information on living donor outcomes.

Similarly, the "European Standards of Quality and Safety of Organs Intended for Transplantation" states that adequate follow-up is part of internationally recognized measures aimed at protecting living kidney donors and ensuring the quality and safety of organ donation. ${ }^{524}$

\section{Content of Follow-Up}

Follow-up care after kidney donation should focus on the monitoring and maintenance of general and kidney health including reinforcement of healthy lifestyle practices (eg, healthy dietary habits, maintenance of healthy weight, regular aerobic exercise), avoidance of potentially nephrotoxic exposures (eg, tobacco use, nonsteroidal anti-inflammatory drugs, nephrotoxic medications), prevention of diseases that may cause CKD (eg, hypertension, diabetes mellitus, CVD), and timely management of such diseases if they develop after donation. As discussed in chapter 10, hypertension is diagnosed more commonly in kidney donors than in persons with comparable baseline health, although this may in part reflect greater monitoring and earlier recognition. ${ }^{24,229}$ Early detection and treatment of medical conditions that may subsequently affect GFR may protect the donor from further loss of GFR or other deterioration in health. Such conditions should be managed according to clinical practice guidelines either by the donor's primary care provider, or at the transplant center if appropriate care is available.

Because many donors develop low GFR that meets criteria for diagnosis of CKD and the risk of someday developing kidney failure needing treatment with dialysis or transplantation is slightly higher as a result of donation (see chapter 5), ${ }^{30,32}$ postdonation monitoring for kidney disease and CKD risk factors is warranted. Living kidney donors should be monitored for CKD and managed according to the 2012 KDIGO CKD Guideline. ${ }^{124}$ Donors should also receive age-appropriate healthcare maintenance, and management of clinical conditions and health risk factors according to clinical practice guidelines for the local or regional population.

Assessment of psychosocial metrics such as HRQoL have been recommended as part of postdonation follow-up to 
monitor general well-being, ${ }^{525}$ and to help transplant centers identify donors at risk for poor psychosocial outcomes. ${ }^{414,526}$ Quality of life measures are collected as part of standard postdonation follow-up in some donor registries. $^{445,527}$ Guidelines for metric thresholds indicating presence of an impairment that would prompt more attention by the clinician are not specifically defined in donors, but could be based on existing test standards (eg, scores below 0.50 standard deviation of the normative mean on the "Short Form" (SF) class of measure, SF-36, 12, or 8).

\section{Follow-Up Timing and Processes}

As part of the informed consent process for donation, transplant centers should educate donor candidates on the importance of follow-up, disclose donor responsibilities and potential costs of follow-up participation, and develop a personalized follow-up plan. Because donors in many countries report regular follow-up with a primary provider, ${ }^{528}$ donor follow-up and care may be appropriately performed by a primary care provider to preserve convenience for the donor. However, communication of follow-up information back to the transplant center is necessary for centers to be aware of the health status of their donors, to comply with reporting mandates (when applicable), and to direct additional care if needed. The role of a primary care provider in a donor's follow-up plan should be established before donation. The transplant center should always be available as a resource for the donor's primary care provider. Documentation of a donor's acknowledgement of their responsibility to participate in follow-up through a signed care plan has been proposed as part of education, ${ }^{525}$ although efficacy in achieving follow-up participation has not been formally evaluated. Particular attention should be given to donors with risk factors for follow-up deficiencies and to those with baseline factors associated with increased risk of CKD over time.

Donor education before and at the time of donation is critical to support an informed donation decision, but there is a need to continue education on health promoting practices as the donor recovers and ages. A donor in the midst of making a decision to donate or dealing with the early outcomes of donation such as postoperative pain and financial pressures may not absorb all the information on long-term issues.

The establishment of standardized follow-up at serial time points provides a pattern for follow-up care that the donor may be more likely to continue on regular, yearly preventive healthcare visits. ${ }^{529}$ Regular contact between the transplant center and the donor and/or the donor's primary care physician also increases familiarity of staff with issues that develop after donation, providing an opportunity to modify education materials or processes to better manage these situations for future donors. While regular healthcare maintenance is important over the lifetime after donation, data collection at regular time points by transplant centers should be pertinent to donation, attainable, and not overly burdensome on the donor or the transplant center.

Early follow-up of donors by transplant centers is mandated in some countries including the United States, Canada and Australia. ${ }^{47,49,73}$ In the United States, the country that performs the largest volume of living donor kidney transplants per year, follow-up reporting by centers to the national transplant registry is limited to 2 years postdonation, and has historically suffered from missing data and frequent loss-to-follow-up..$^{73,530-532}$ Follow-up deficiencies have correlated with patient factors including younger donor age, black race, lack of insurance, lower educational attainment, and greater distance to the transplant center. $^{528,530,531}$ Lack of health insurance had been more common among living kidney donors in the United States than in the general US population, although that disparity has not been present in recent years, perhaps due to changes in the economy and policy changes including the Patient Protection and Affordable Care Act (ACA). ${ }^{533,534}$ Lack of insurance and lower educational attainment may pose lesser obstacles to follow-up in other countries where national healthcare is available. Among US centers in 2008 to 2012, large annual living donation volumes were also associated with increased rates of follow-up deficiencies. ${ }^{530}$ However, implementation of mandatory thresholds for collection of postdonation clinical and laboratory data in the United States in 2013 with regulatory implications has led to improved rates of early follow-up data collection, and high levels of follow-up have been achieved by programs across the volume spectrum. ${ }^{530,535}$

There is a real need for comprehensive, long-term (lifetime) follow-up of living donors. Short-term follow-up may detect severe adverse outcomes early after donation but cannot detect gradual, progressive complications that occur late after donation. Clinically important events that may develop in the intermediate to long-term after donation include hypertension, CKD, metabolic conditions (eg, diabetes mellitus, hyperlipidemia) and CVD, which in turn may increase the risk of kidney failure and mortality.

Transplant programs achieve high rates of postdonation follow-up have practices that appear to be grounded in: 1) program conviction that follow-up is essential for donor safety and well-being; 2) emphasis on building and maintaining a relationship with each donor; 3 ) use of a systematic approach to follow-up; and 4) efforts to minimize the burden on donors. ${ }^{525}$ Open-ended, qualitative interviews of US programs found that programs with high rates of successful follow-up endorsed all 4 of these concepts. ${ }^{525}$ Recent single-center experience supports that institution of follow-up performance improvement initiatives with dedicated program resources is financially feasible and can result in more accurate and complete follow-up after donation. ${ }^{536}$

\section{Donor Registries}

When national or regional donor registries exist, capture of donor follow-up information, either through center reporting or direct donor contacts, facilitates data aggregation, assessment and dissemination of current donor outcomes data. Informed consent for collection of follow-up information for registry reporting should be requested during the consent for donor evaluation. In countries where registries do not exist, efforts should be made to establish and maintain a donor registry to support ongoing monitoring of postdonation outcomes, as the characteristics of accepted donors and care practices may evolve over time.

Some countries have instituted systematic approaches to longer-term living donor follow-up and registry reporting. The EULID project began with an 11-nation assessment of living donation practices in Europe that recommended mandatory living donor registration and follow-up data collections through a centralized donor database system, and 
mandatory regulatory audits at the national and center level. ${ }^{444}$ An online registry is used for reporting of information on donor health status, quality of informed consent, and psychosocial and socioeconomic outcomes. The European Living Donor Psychosocial Follow-Up (ELIPSY), a study conducted in 6 centers of different European countries (Spain, Germany, France, Portugal, Sweden and Turkey) in 2009 to 2012, focused on monitoring donor psychosocial well-being and quality of life. ${ }^{527}$

The SOL-DHR provides a model for involvement of primary care physicians to achieve short- and long-term follow-up of donor health and well-being. ${ }^{445}$ In 1993, a national protocol was initiated for all Swiss transplant centers to organize lifelong follow-up after donation at $1,3,5$, 7,10 years, and biennially thereafter, by sending a package to the donor asking the donor to make an appointment with the family physician of their choice. The package contains brief information for the donor and the family physician, a health questionnaire, tubes for blood and urine samples, and a prepaid envelope for sending the samples at room temperature to the central laboratory. The basic biennial follow-up questionnaire is completed by the family physician. In 2002, requests were added for completion an additional 8-Item Short-Form (SF-8) and social-status questionnaire by the donor. Nonresponses are followed by contacting the recipient, the donor's health insurance and public registries to identify whether the donor has died and, if so, the cause of death. Lifelong follow-up of the living donor's health status by centers is required by the Swiss Transplant Law. Although donors may choose to stop participating, compliance is promoted by informing donors about the aims of the protocol and the registry before their donation. Similarly, in Norway, donor follow-up includes initial contacts at weeks 3 to 4 , month 3 , then yearly monitoring for 5 years, and then lifelong contacts every fifth year thereafter. Clinical assessment of donors including BP, blood tests and urinalysis examinations is performed by local county hospitals. Durability of follow-up has been demonstrated as $99,95,84$, and $77 \%$ of donors are still seen by local nephrologists at $1,5,10$, and 15 years follow-up, respectively (Dr. Hallvard Holdaas, personal communication).

In 2017, the US Scientific Registry of Transplant Recipients began planning and transplant program enrollment for a Health Services Research Administration "Living Donor Collective" pilot project that seeks to achieve life-long living donor follow-up through direct contacts by staff and novel data linkages. A notable feature of the Collective is a plan to collect long-term outcomes data on all persons who present for donor candidate evaluation to provide information on donor exclusion practices and identify a set of healthy controls (eg, approved donors who do not donate due to recipient exclusions.) If successful, the goal is to expand to national participation. ${ }^{537}$

Given the current limited scope and duration of donor registries in many countries, and the possibility that some donors as independent decision makers may choose not to stay in contact with their transplant center, collection of outcome data in dedicated, funded research studies, and integration of donor registry data with other information sources such as health administrative data, ESKD and mortality registries are also important additional priorities for advancing understanding of long-term health outcomes after donation. ${ }^{538}$

\section{What Prior Guidelines Recommend}

The British Transplantation Society and The Renal Association UK Guidelines for Living Donor Kidney Transplantation state that: ${ }^{48,499}$

- Life-long follow-up is recommended after donor nephrectomy. For donors who are resident in the United Kingdom, this should be offered locally or at the transplant center according to the wishes of the donor, but such arrangements must facilitate the collection of data for submission to the UK Living Donor Registry on long-term morbidity and mortality. Donors from overseas who travel to the United Kingdom to donate are not entitled to follow-up in the United Kingdom but should be given advice about appropriate follow-up before returning to their country of origin (C1).

- Arrangements must be made to ensure that the unsuitable donor, who is unable to proceed to donation, is appropriately followed up and referred for further investigation and management (B1).

- National Health Service Blood and Transplant has given a formal undertaking that any living kidney donor who develops kidney failure in the perioperative period as a consequence of donation will receive priority for a deceased donor kidney transplant (not graded).

Although it was not a specific guideline recommendation and no evidence was cited, the Amsterdam Forum suggested that "As in the general population, based on age and other medical risk factors (eg, hypertension, proteinuria, hyperlipidemia, impaired glucose tolerance test), kidney donors should undergo regular long-term follow-up of body weight, BP, blood glucose, serum creatinine, and urinalysis." ${ }^{38}$ In the United States, OPTN policy enacted in 2013 requires transplant centers to collect and report the following information on their donors to the national registry at 6 months, 1 year, and 2 years postdonation: serum creatinine, urine protein and a set of clinical outcomes (eg, kidney complications, need for maintenance dialysis, development of hypertension or diabetes, loss of insurance due to donation). ${ }^{73}$ Other guidelines also suggest postdonation follow-up for at least 1 year ${ }^{49}$ or lifelong ${ }^{47,55}$ and provision of donation-related psychosocial services as needed during follow-up have also been proposed. ${ }^{47,409,498}$ As discussed in chapter 18 (Policy), living donors who develop ESKD in the United States receive priority for deceased donor kidney allocation. ${ }^{512}$

\section{RESEARCH RECOMMENDATIONS}

- Develop communication and integrated care models to:

- Examine electronic tools such as websites or portals to maintain contact with donors, facilitate data collection, and provide messaging to disseminate educational information to donors. 522

- Formulate strategies for using inter-institution-compatible electronic medical records to facilitate transmission of donor follow-up information, such as clinical data from care encounters, directly to national registries. ${ }^{531}$

- Formulate and assess the outcomes of center-based initiatives to provide long-term donor follow-up and support through integrated laboratory and clinical monitoring, expansion of preventive health strategies, and fostering of peer education through social support networks between past, current and future donors. 539

- Establish, improve and integrate national/international donor registries to facilitate capture and analysis of long-term 
outcomes information for large representative samples of living donors to: (1) address knowledge gaps related to the long-term consequences of donation, (2) provide data to inform donor selection criteria, (3) support quality assurance and program improvement at transplant centers, and 4) sustain and strengthen public confidence in the practice of living donation. 523,531

- Develop HRQoL metric thresholds to identify the presence of an impairment in postdonation psychosocial well-being warranting closer attention by the clinician

- Improve and assess the efficacy of educational resources to promote sustained healthy lifestyle choices and behaviors, and participation in regular postdonation follow-up and care, such as via newsletters, links to transplant center health recommendations or national guideline website documents.

- Develop and assess strategies for communicating new information that differs from what a donor was told before donation, to past donors and their physicians.

\section{ACKNOWLEDGMENTS}

A special debt of gratitude is owed to the KDIGO cochairs, David C. Wheeler and Wolfgang C. Winkelmayer, and the KDIGO Executive Committee for their invaluable guidance throughout the development of this guideline. In particular, the authors thank the ERT members for their substantial contribution to the rigorous assessment of the available evidence. The authors are also especially grateful to the WG members for their expertise throughout the entire process of literature review, meeting participation, the critical writing and editing of the statements and rationale, which made the publication of this guideline possible. The generous gift of their time and dedication is greatly appreciated. KDIGO gratefully acknowledges the valuable contributions from CKD-Prognosis Consortium and its members for their meta-analyses which serve as the basis for the donor selection framework presented in this report: Josef Coresh, $\mathrm{MD}, \mathrm{PhD}$, Morgan E. Grams, MD, PhD, Yingying Sang, MS, Kunihiro Matsushita, MD, PhD, Shoshana Ballew, PhD, Alex R. Chang, MD, Eric K. H, Chow MSc, Csaba P. Kovesdy, MD, Girish N. Nadkarni, MD, MPH, Varda Shalev, MD, MPA. KDIGO also thanks the following individuals who provided valuable feedback: Daniel C. Brennan, MD, Mary Amanda Dew, PhD, Robert Gaston, MD, Elisa J. Gordon, PhD, MPH, Rebecca Hays, MSW, Peter C. Harris, PhD, York Pei, MD, Emilio Poggio, MD, Peter P. Reese, MD, MSCE, Robert Steiner, MD, Christie P. Thomas, MD, Roser Torra, MD, $\mathrm{PhD}$, Vicente E. Torres, $\mathrm{MD}, \mathrm{PhD}$, and Matthew Weir, MD. KDIGO also thanks Josep M. Campistol, MD, PhD, José Osmar Medina Pestana, MD, PhD, and Faissal A. Shaheen, MD for their initial contributions to the guideline scope.

Finally, and on behalf of the WG, the authors gratefully acknowledge the careful assessment of the draft guideline by external reviewers. The WG considered all of the valuable comments made and, where appropriate, suggested changes were incorporated into the final publication. The following individuals provided feedback during the public review of the draft guideline: Mario Abbud-Filho; Sadiq Abdul Baqi; ML Aguera; Abdulnaser Alabadi; Bülent Altun; Alesssandro Amore; Andrea Angioi; Mariano Arriola; Nancy Ascher; Gloria Ashuntantang; Phillippa Bailey; Peter Barany; Zunaid Barday; André Barreto Pereira; Rashad Barsoum; Rommel Bataclan; Don Batisky; Leticia Belmont-Martinez; Roberta Billman; Annie-Claude Blouin; Yassine Bouatou; Neil Boudville;
Diane Brockington; Patricia Campbell; Marcelo Cantarovich; Jianghua Chen; Xingxing Cheng; Attasit Chokechanachaisakul; Rolando Claure-Del Granado; Shandie Covington; Ana Cusumano; Declan de Freitas; Goffredo Del Rosso; Francis Delmonico; Mary Amanda Dew; Nida Dincel; Christine Dipchand; Edmar Elcarte; Josette Eris; Stephen Fadem; Peter Ferenci; Sandro Feriozzi; Sebastião Ferreira-Filho; Frances Flinter; Sue Gagne; Alvaro Garcia Garcia; Mike Germain; Christiane Geuer; Osama Gheith; Matthias Girndt; Richard Glassock; Paul Goodyer; Elisa Gordon; William Gourlay; Jared Grantham; Rebecca Hays; Darlene Heisler; Macey Henderson; Seyed M Hosseini-Moghaddam; Osama Hrith; Salwa Ibrahim; Ikponmwosa Iyawe; Anthony Jevnikar; Chandra Mauli Jha; Vivek Jha; Malek Kamoun; Nada Kanaan; Clifford Kashtan; Thomas Kelly; Bryce Kiberd; Hyoung Tae Kim; David Klassen; Nine Knoers; Dirk Kuypers; David Landsberg; Po Chang Lee; Edgar Lerma; Marie Ludlow; Mitra Mahdavi-Mazdeh; Martin Mai; Shafi Malik; Francesca Mallamaci; Carlos Marroquin; Jennifer Martin; Thomas McGregor; Dianne McKay; Enisa Mesic; Piergiorgio Messa; Renzo Mignani; Euclides Briones Morales; Eugen Mota; Mark Murphy; Abimereki Muzaale; Charles Newstead; Abdou Niang; Maurizio Nordio; Uday Nori; Philip O'Connell; Juha Peräsaari; André Pereira; Gerson Marques Pereira Jr; Ronald Perrone; Carlos Poli-de-Figueiredo; Claudio Ponticelli; Pradeep Kumar Rai; Harun Rashid; Praveen Ratanasrimetha; Cibele Rodrigues; Jacques Rottembourg; Shaifali Sandal; Richard Sandford; Craig Schmidt; Silvi Shah; Deepak Sharma; Risa Simon; Neeraj Singh; Habib Skhiri; Robert Steiner; Sylvia Stracke; Rodrigo Tagle; Seng Hoe Tan; Ekamol Tantisattamo; Mihály Tapolyai; Gabor Telkes; Christie Thomas; Lee Anne Tibbles; Vali Toth; Nico Tronchet; Teresa Trottman; Yusuke Tsukamoto; Albertien M. van Eerde; Evgueniy Vazelov; Anitha Vijayan; Rafael Weissheimer; Kerstin Westman; Christine White; Jean-Luc Wolff; Mai-Szu Wu; Chulwoo Yang; Jane Zill; Carmine Zoccali.

Participation in the review does not necessarily constitute endorsement of the content of this report by the above individuals, or the organization or institution they represent.

Amit X. Garg, MD, PhD

Krista L. Lentine, MD, PhD

Work Group Cochairs

\section{REFERENCES}

1. Uhlig K, Macleod A, Craig J, et al. Grading evidence and recommendations for clinical practice guidelines in nephrology. A position statement from Kidney Disease: Improving Global Outcomes (KDIGO). Kidney Int. 2006;70:2058-2065.

2. Ethics Committee of the Transplantation Society. The consensus statement of the Amsterdam Forum on the Care of the Live Kidney Donor. Transplantation 2004;78:491-492.

3. Slinin Y, Brasure M, Eidman K, et al. Long-term outcomes of living kidney donation. Transplantation. 2016;100:1371-1386.

4. Lentine KL, Segev DL. Understanding and communicating medical risks for living kidney donors: a matter of perspective. J Am Soc Nephrol. 2017;28:12-24.

5. White CM, Ip S, McPheeters M, et al. Using existing systematic reviews to replace de novo processes in conducting comparative effectiveness reviews. In: Methods Guide for Effectiveness and Comparative Effectiveness Reviews [Internet]. Rockville (MD): Agency for Healthcare Research and Quality (US); 2008-.AHRQ Methods for Effective Health Care; 2009. 
6. Viswanathan M, Berkman ND, Dryden DM, et al. Assessing risk of bias and confounding in observational studies of interventions or exposures: further development of the RTI item bank. AHRQ Methods for Effective Health Care: Rockville (MD); 2013.

7. Grams ME, Sang Y, Levey AS, et al. Kidney-failure risk projection for the living kidney-donor candidate. N Engl J Med. 2016;374:411-421.

8. Huang N, Foster MC, Lentine KL, et al. Estimated GFR for living kidney donor evaluation. Am J Transplant. 2016;16:171-180.

9. Guyatt $\mathrm{GH}$, Oxman AD, Kunz R, et al. Going from evidence to recommendations. BMJ. 2008;336:1049-1051.

10. Atkins D, Best D, Briss PA, et al. Grading quality of evidence and strength of recommendations. BMJ. 2004;328:1490.

11. Guyatt GH, Schunemann HJ, Djulbegovic B, et al. Guideline panels should not GRADE good practice statements. J Clin Epidemiol. 2015; 68:597-600

12. AGREE Collaboration. Development and validation of an international appraisal instrument for assessing the quality of clinical practice guidelines: the AGREE project. Qual Saf Health Care 2003;12:18-23.

13. Shiffman RN, Shekelle P, Overhage JM, et al. Standardized reporting of clinical practice guidelines: a proposal from the Conference on Guideline Standardization. Ann Intern Med. 2003;139:493-498

14. Institute of Medicine. Finding what works in health care: Standards for systematic reviews. The National Academies Press: Washington, DC, 2011.

15. Institute of Medicine. Clinical practice guidelines we can trust. The National Academies Press: Washington, DC, 2011.

16. Thiessen C, Gordon EJ, Reese PP, et al. Development of a donorcentered approach to risk assessment: rebalancing nonmaleficence and autonomy. Am J Transplant. 2015;15:2314-2323.

17. Sixty-Third World Health Assembly. WHA63.22. Agenda item 11.21, 21 May 2010 Human organ and tissue transplantation. Available at: http:// apps.who.int/gb/ebwha/pdf_files/WHA63/A63_R22-en.pdf. (Accessed: September 7, 2016)

18. Abecassis $M$, Adams $M$, Adams $P$, et al. Consensus statement on the live organ donor. JAMA. 2000;284:2919-2926.

19. Tong A, Chapman JR, Wong G, et al. Living kidney donor assessment: challenges, uncertainties and controversies among transplant nephrologists and surgeons. Am J Transplant. 2013;13:2912-2923.

20. Tong A, Chapman JR, Wong G, et al. Screening and follow-up of living kidney donors: a systematic review of clinical practice guidelines. Transplantation. 2011;92:962-972.

21. Cozzi E, Biancone L, López-Fraga M, et al. long-term outcome of living kidney donation: position paper of the European Committee on Organ Transplantation, Council of Europe. Transplantation. 2016;100: 270-271.

22. Reese PP, Caplan AL, Kesselheim AS, et al. Creating a medical, ethical, and legal framework for complex living kidney donors. Clin J Am Soc Nephrol. 2006;1:1148-1153.

23. Segev DL, Muzaale AD, Caffo BS, et al. Perioperative mortality and long term survival following live kidney donation. JAMA. 2010;303:959-966.

24. Matas AJ, Bartlett ST, Leichtman AB, et al. Morbidity and mortality after living kidney donation, 1999-2001: survey of United States transplant centers. Am J Transplant. 2003;3:830-834.

25. Hadjianastassiou VG, Johnson RJ, Rudge CJ, et al. 2509 living donor nephrectomies, morbidity and mortality, including the UK introduction of laparoscopic donor surgery. Am J Transplant. 2007;7:2532-2537.

26. Young A, Storsley L, Garg AX, et al. Health outcomes for living kidney donors with isolated medical abnormalities: a systematic review. Am J Transplant. 2008;8:1878-1890.

27. Lafranca JA, Hagen SM, Dols LF, et al. Systematic review and metaanalysis of the relation between body mass index and short-term donor outcome of laparoscopic donor nephrectomy. Kidney Int. 2013:83:931-939.

28. Schold JD, Goldfarb DA, Buccini LD, et al. Comorbidity burden and perioperative complications for living kidney donors in the United States. Clin J Am Soc Nephrol. 2013;8:1773-1782.

29. Lentine KL, Lam NN, Axelrod D, et al. Perioperative complications after living kidney donation: a national study. Am J Transplant. 2016;16: 1848-1857.

30. Muzaale AD, Massie AB, Wang MC, et al. Risk of end-stage renal disease following live kidney donation. JAMA. 2014;311:579-586.

31. Anjum S, Muzaale AD, Massie AB, et al. Patterns of end-stage renal disease caused by diabetes, hypertension, and glomerulonephritis in live kidney donors. Am J Transplant. 2016;16: 3540-3547.

32. Mjoen G, Hallan S, Hartmann A, et al. Long-term risks for kidney donors. Kidney Int. 2014;86:162-167.
33. Lam NN, Lentine KL, Garg AX. End-stage renal disease risk in live kidney donors: what have we learned from two recent studies? Curr Opin Nephrol Hypertens. 2014;23:592-596.

34. Lam NN, Lentine KL, Levey AS, et al. Long-term medical risks to the living kidney donor. Nat Rev Nephrol. 2015;11:411-419.

35. Kiberd BA, Clase CM. Cumulative risk for developing end-stage renal disease in the US population. J Am Soc Nephrol. 2002;13: 1635-1644.

36. Steiner RW. The risks of living kidney donation. N Engl J Med. 2016; 374:479-480

37. Grams ME, Garg AX, Lentine KL. Kidney-failure risk projection for the living kidney-donor candidate. N Engl J Med. 2016;374:2094-2095.

38. Delmonico F, Council of the Transplantation Society. A report of the Amsterdam forum on the care of the live kidney donor: data and medical guidelines. Transplantation. 2005;79:S53-S66.

39. Hays RE. Informed consent of living kidney donors: pitfalls and best practice. Curr Transpl Rep. 2015;2:29-34

40. Thiessen C, Kim YA, Formica R, et al. Written informed consent for living kidney donors: practices and compliance with CMS and OPTN requirements. Am J Transplant. 2013;13:2713-2721.

41. Valapour M, Kahn JP, Bailey RF, et al. Assessing elements of informed consent among living donors. Clin Transplant. 2011;25:185-190.

42. Gordon EJ. Informed consent for living donation: a review of key empirical studies, ethical challenges and future research. Am J Transplant. 2012;12:2273-2280

43. Housawi AA, Young A, Boudville N, et al. Transplant professionals vary in the long-term medical risks they communicate to potential living kidney donors: an international survey. Nephrol Dial Transplant. 2007;22: 3040-3045.

44. Rodrigue JR, Pavlakis M, Danovitch GM, et al. Evaluating living kidney donors: relationship types, psychosocial criteria, and consent processes at US transplant programs. Am J Transplant. 2007;7:2326-2332.

45. Gordon EJ, Butt Z, Jensen SE, et al. Opportunities for shared decision making in kidney transplantation. Am J Transplant. 2013;13:1149-1158.

46. Dew MA, Jacobs CL, Jowsey SG, et al. Guidelines for the psychosocial evaluation of living unrelated kidney donors in the United States. Am J Transplant. 2007;7:1047-1054.

47. Canadian Council for Donation and Transplantation. Enhancing living donation: a Canadian forum-report and recommendations, February 9-12, 2006, Vancouver, British Columbia.

48. The British Transplantation Society and The Renal Association. The United Kingdom guidelines for living donor kidney transplantation. Third Edition, 2011. Available at: https://bts.org.uk/wp-content/uploads/2016/09/19_BTS_RA_Living_Donor_Kidney-1.pdf. (Accessed: September 7, 2016)

49. National Health and Medical Research Council. Organ and tissue donation by living donors. Guidelines for ethical practice for health professionals, 2007. Available at: https://www.nhmrc.gov.au/guidelines-publications/ e75. (Accessed: September 7, 2016).

50. Abramowicz D, Cochat P, Claas FH, et al. European Renal Best Practice Guideline on kidney donor and recipient evaluation and perioperative care. Nephrol Dial Transplant. 2015;30:1790-1797.

51. OPTN (Organ Procurement and Transplantation Network)/UNOS (United Network for Organ Sharing). OPTN Policies, Policy 14: Living Donation. http://optn.transplant.hrsa.gov/govemance/policies/. (Accessed: June 28, 2017).

52. Gesetz über die Spende, Entnahme und Übertragung von Organen. [Legislation on the donation, harvesting and transplantation of organs] (Transplantationsgesetz - TPG) From November 5, 1997 (Bundesgesetzblatt I page 2631).

53. Campbell M, Wright L, Greenberg RA, et al. How young is too young to be a living donor? Am J Transplant. 2013;13:1643-1649.

54. Spanish Society of Nephrology (S.E.N.) and Spanish Transplant Organisation (ONT). Recommendations for living-donor kidney transplantation. Nefrologia 2010;30:1-105

55. Kälble T, Lucan M, Nicita G, et al. EAU guidelines on renal transplantation. Eur Urol. 2005;47:156-166.

56. Living Donor Informed Consent Checklist. Available at: https://optn. transplant.hrsa.gov/resources/living-donation. (Accessed: June 29, 2017).

57. Plain language informed consent policy summary (English and Spanish). Available at: https://optn.transplant.hrsa.gov/resources/living-donation. (Accessed: June 29, 2017).

58. Tan JC, Gordon EJ, Dew MA, et al. Living donor kidney transplantation: facilitating education about live kidney donation—recommendations from a consensus conference. Clin J Am Soc Nephrol. 2015;10:1670-1677. 
59. European Best Practice Guidelines for Renal Transplantation. Section II: Evaluation and selection of donors. Nephrol Dial Transplant 2000;15:39-51.

60. Melcher ML, Blosser CD, Baxter-Lowe LA, et al. Dynamic challenges inhibiting optimal adoption of kidney paired donation: findings of a consensus conference. Am J Transplant. 2013;13:851-860.

61. OPTN (Organ Procurement and Transplantation Network)/UNOS (United Network for Organ Sharing). OPTN Policies, Policy 13: Kidney paired donation (KPD). Available at: http://optn.transplant.hrsa.gov/governance/ policies. (Accessed: June 28, 2017).

62. Mamode N, Lennerling A, Citterio F, et al. Anonymity and live-donor transplantation: an ELPAT view. Transplantation. 2013;95:536-541.

63. Bingaman AW, Wright FH Jr, Kapturczak M, et al. Single-center kidney paired donation: the Methodist San Antonio experience. Am J Transplant. 2012;12:2125-2132.

64. Hendren E, Gill J, Landsberg D, et al. Willingness of directed living donors and their recipients to participate in kidney paired donation programs. Transplantation. 2015;99:1894-1899.

65. Cuffy MC, Ratner LE, Siegler M, et al. Equipoise: ethical, scientific, and clinical trial design considerations for compatible pair participation in kidney exchange programs. Am J Transplant. 2015;15:1484-1489.

66. Young A, Kim SJ, Gibney EM, et al. Discovering misattributed paternity in living kidney donation: prevalence, preference, and practice. Transplantation. 2009;87:1429-1435.

67. Ross LF. Good ethics requires good science: why transplant programs should not disclose misattributed parentage. Am J Transplant. 2010; 10:742-746.

68. Wright L, MacRae S, Gordon D, et al. Disclosure of misattributed paternity: issues involved in the discovery of unsought information. Semin Dial. 2002;15:202-206.

69. Mataya L, Meadow J, Thistlethwaite JR Jr, et al. Disclosing health and health behavior information between living donors and their recipients. Clin J Am Soc Nephrol. 2015;10:1609-1616.

70. Hizo-Abes P, Young A, Reese PP, et al. Attitudes to sharing personal health information in living kidney donation. Clin J Am Soc Nephrol. 2010;5:717-722.

71. Seem DL, Lee I, Umscheid CA, et al. Excerpt from PHS guideline for reducing HIV, HBV and HCV transmission through organ transplantation. Am J Transplant. 2013;13:1953-1962.

72. Rodrigue JR, Ladin K, Pavlakis M, et al. Disclosing recipient information to potential living donors: preferences of donors and recipients, before and after surgery. Am J Transplant. 2011;11:1270-1278.

73. OPTN (Organ Procurement and Transplantation Network)/UNOS (United Network for Organ Sharing). OPTN Policies, Policy 18: Data submission requirements. http://optn.transplant.hrsa.gov/governance/policies/. (Accessed: June 28, 2017)

74. Danovitch GM, Chapman J, Capron AM, et al. Organ trafficking and transplant tourism: the role of global professional ethical standards-the 2008 Declaration of Istanbul. Transplantation. 2013;95:1306-1312.

75. Allen MB, Abt PL, Reese PP. What are the harms of refusing to allow living kidney donation? An expanded view of risks and benefits. Am J Transplant. 2014;14:531-537.

76. Agency for Healthcare Research and Quality: The SHARE Approach—Using the Teach-Back technique: A reference guide for health care providers. http://www.ahrq.gov/professionals/education/ curriculum-tools/shareddecisionmaking/tools/tool-6/index.html (Access date: May 4, 2016)

77. Gordon EJ, Mullee J, Butt Z, et al. Optimizing informed consent in living liver donors: evaluation of a comprehension assessment tool. Liver Transpl. 2015;21:1270-1279.

78. Rodrigue JR, Vishnevsky T, Fleishman A, et al. Patient-reported outcomes following living kidney donation: a single center experience. J Clin Psychol Med Settings. 2015;22:160-168.

79. Faden RR, Beauchamp T. A history and theory of informed consent. New York: Oxford University Press; 1986.

80. Millis MA, Simmerling M. Prisoners as organ donors: is it worth the effort? Is it ethical? Transplant Proc. 2009;41:23-24.

81. Ross LF. What the medical excuse teaches us about the potential living donor as patient. Am J Transplant. 2010;10:731-736.

82. Thiessen C, Kim YA, Formica R, et al. Opting out: confidentiality and availability of an 'alibi' for potential living kidney donors in the USA. J Med Ethics. 2015;41:506-510.

83. Maple NH, Hadjianastassiou V, Jones R, et al. Understanding risk in living donor nephrectomy. J Med Ethics. 2010;36:142-147.
84. Fortin MC, Buchman D, Wright L, et al. Public solicitation of anonymous organ donors: a position paper by the Canadian Society of Transplantation. Transplantation. 2017;101:17-20.

85. Cook RI, Wreathall J, Smith A, et al. Probabilistic risk assessment of accidental $\mathrm{ABO}$-incompatible thoracic organ transplantation before and after 2003. Transplantation. 2007:84:1602-1609.

86. Gloor JM, Lager DJ, Moore SB, et al. ABO-incompatible kidney transplantation using both A2 and non-A2 living donors. Transplantation. 2003;75:971-977.

87. Bachelet T, Martinez C, Del Bello A, et al. Deleterious impact of donorspecific anti-HLA antibodies toward HレA-CW and HLA-DP in kidney transplantation. Transplantation. 2016;100:159-166.

88. Filippone EJ, Farber JL. Humoral immune response and allograft function in kidney transplantation. Am J Kidney Dis. 2015;66:337-347.

89. Tait BD, Süsal $\mathrm{C}$, Gebel HM, et al. Consensus guidelines on the testing and clinical management issues associated with HLA and non-HLA antibodies in transplantation. Transplantation. 2013;95:19-47.

90. Segev DL, Gentry SE, Melancon JK, et al. Characterization of waiting times in a simulation of kidney paired donation. Am J Transplant. 2005, 5:2448-2455

91. Montgomery JR, Berger JC, Warren DS, et al. Outcomes of ABOincompatible kidney transplantation in the United States. Transplantation. 2012;93:603-609.

92. Montgomery RA, Lonze BE, King KE, et al. Desensitization in HLAincompatible kidney recipients and survival. N Engl J Med. 2011;365: 318-326.

93. Segev DL, Gentry SE, Warren DS, et al. Kidney paired donation and optimizing the use of live donor organs. JAMA. 2005;293:1883-1890.

94. Matas AJ, Smith JM, Skeans MA, et al. OPTN/SRTR 2013 Annual Data Report: kidney. Am J Transplant. 2015;15(Suppl 2):1-34.

95. de Klerk M, Haase-Kromwijk BJ, Witvliet M, et al. [Favourable results of the first 2 years of the Dutch paired, living donor, kidney exchange programme]. Ned Tijdschr Geneeskd. 2007;151:130-133.

96. Segev DL, Veale JL, Berger JC, et al. Transporting live donor kidneys for kidney paired donation: initial national results. Am J Transplant. 2011;11: 356-360.

97. Treat EG, Miller ET, Kwan L, et al. Outcomes of shipped live donor kidney transplants compared with traditional living donor kidney transplants. Transpl Int. 2014:27:1175-1182.

98. Melcher ML, Leeser DB, Gritsch HA, et al. Chain transplantation: initial experience of a large multicenter program. Am J Transplant. 2012;12: 2429-2436.

99. Aikawa A, Kawamura T, Shishido S, et al. ABO-incompatible livingdonor pediatric kidney transplantation in Japan. Clinics (Sao Paulo). 2014;69(Suppl 1):22-27.

100. Aikawa A, Saito K, Takahashi K. Trends in ABO-incompatible kidney transplantation. Exp Clin Transplant. 2015:13(Suppl 1):18-22.

101. Takahashi K, Saito K, Takahara S, et al. Excellent long-term outcome of ABO-incompatible living donor kidney transplantation in Japan. Am J Transplant. 2004;4:1089-1096.

102. Lentine $\mathrm{KL}$, Axelrod D, Klein $\mathrm{C}$, et al. Early clinical complications after ABO-incompatible live-donor kidney transplantation: a national study of Medicare-insured recipients. Transplantation. 2014:98:54-65.

103. Ishikawa N, Yagisawa T, Kimura T, et al. Kidney transplantation of living unrelated and $\mathrm{ABO}$-incompatible donor-recipient combinations. Transplant Proc. 2013;45:1242-1244.

104. Kong JM, Ahn J, Park JB, et al. ABO incompatible living donor kidney transplantation in Korea: highly uniform protocols and good mediumterm outcome. Clin Transplant. 2013;27:875-881.

105. Montgomery RA, Locke JE, King KE, et al. ABO incompatible renal transplantation: a paradigm ready for broad implementation. Transplantation. 2009;87:1246-1255

106. Opelz G, Morath C, Süsal C, et al. Three-year outcomes following 1420 ABO-incompatible living-donor kidney transplants performed after $\mathrm{ABO}$ antibody reduction: results from 101 centers. Transplantation. 2015; 99:400-404.

107. OPTN (Organ Procurement and Transplantation Network)/UNOS (United Network for Organ Sharing). National Data Reports. https://optn. transplant.hrsa.gov/data/view-data-reports/national-data/. (Accessed June 28, 2017)

108. Axelrod D, Segev DL, Xiao H, et al. Economic impacts of ABOincompatible live donor kidney transplantation: A national study of Medicare-insured recipients. Am J Transplant. 2016;16: $1465-1473$ 
109. Orandi BJ, Luo X, Massie AB, et al. Survival benefit with kidney transplants from HLA-incompatible live donors. N Engl J Med. 2016;374: 940-950.

110. Axelrod D, Lentine KL, Schnitzler MA, et al. The incremental cost of incompatible living donor kidney transplant: A national cohort analysis. Am J Transpl. 2017. ePub ahead of print.

111. Clavien PA, Barkun J, de Oliveira ML, et al. The Clavien-Dindo classification of surgical complications: five-year experience. Ann Surg. 2009;250: 187-196.

112. Mjøen G, Øyen $\mathrm{O}$, Holdaas $\mathrm{H}$, et al. Morbidity and mortality in 1022 consecutive living donor nephrectomies: benefits of a living donor registry. Transplantation. 2009;88:1273-1279.

113. Lentine KL, Lam NN, Schnitzler MA, et al. Predonation prescription opioid use: a novel risk factor for readmission after living kidney donation. Am J Transplant. 2017;17:744-753.

114. Cohn SL. Preoperative evaluation for noncardiac surgery. Ann Intern Med. 2016;165:ITC81-ITC96.

115. Fleisher LA, Beckman JA, Brown KA, et al. ACC/AHA 2007 guidelines on perioperative cardiovascular evaluation and care for noncardiac surgery: a report of the American College of Cardiology/American Heart Association Task Force on Practice Guidelines (Writing Committee to Revise the 2002 Guidelines on Perioperative Cardiovascular Evaluation for Noncardiac Surgery): developed in collaboration with the American Society of Echocardiography, American Society of Nuclear Cardiology, Heart Rhythm Society, Society of Cardiovascular Anesthesiologists, Society for Cardiovascular Angiography and Interventions, Society for Vas cular Medicine and Biology, and Society for Vascular Surgery. Circulation. 2007; 116:e418-499.

116. Ferket BS, Genders TS, Colkesen EB, et al. Systematic review of guidelines on imaging of asymptomatic coronary artery disease. J Am Coll Cardiol. 2011:57:1591-1600.

117. Chee YL, Crawford JC, Watson HG, et al. Guidelines on the assessment of bleeding risk prior to surgery or invasive procedures. British Committee for Standards in Haematology. Br J Haematol. 2008;140:496-504.

118. Fernández Fresnedo $G$, de la Oliva Valentín M, Cruzado JM, et al. [Objectives and methodology of S.E.N-ONT guidelines for living donor kidney transplantation]. Nefrologia. 2010;30(Suppl 2):1-2.

119. Devereaux PJ, Mrkobrada M, Sessler DI, et al. Aspirin in patients undergoing noncardiac surgery. N Engl J Med. 2014;370:1494-1503.

120. Guyatt GH, Akl EA, Crowther M, et al. Executive summary: Antithrombotic Therapy and Prevention of Thrombosis, 9th ed: American College of Chest Physicians Evidence-Based Clinical Practice Guidelines. Chest. 2012;141:7S-47S.

121. Qaseem A, Snow V, Fitterman N, et al. Risk assessment for and strategies to reduce perioperative pulmonary complications for patients undergoing noncardiothoracic surgery: a guideline from the American College of Physicians. Ann Intern Med. 2006;144:575-580.

122. American Society of Anesthesiologists Task Force on Perioperative Management of patients with obstructive sleep apnea. Practice guidelines for the perioperative management of patients with obstructive sleep apnea: an updated report by the American Society of Anesthesiologists Task Force on Perioperative Management of patients with obstructive sleep apnea. Anesthesiology 2014;120:268-286.

123. Mills E, Eyawo O, Lockhart I, et al. Smoking cessation reduces postoperative complications: a systematic review and meta-analysis. $\mathrm{Am} J$ Med. 2011;124:144-154 e148.

124. Kidney Disease: Improving Global Outcomes (KDIGO) CKD Work Group. KDIGO 2012 clinical practice guideline for the evaluation and management of chronic kidney disease. Kidney Int 2013:1-150.

125. Earley A, Miskulin D, Lamb EJ, et al. Estimating equations for glomerular filtration rate in the era of creatinine standardization: a systematic review. Ann Intern Med. 2012;156:785-795.

126. Wesson L. Physiology of the Human Kidney. New York: Grune \& Stratton; 1969.

127. Jafar TH, Islam M, Jessani S, et al. Level and determinants of kidney function in a South Asian population in Pakistan. Am J Kidney Dis. 2011;58:764-772

128. Levey AS, Stevens LA, Schmid CH, et al. A new equation to estimate glomerular filtration rate. Ann Intern Med. 2009;150:604-612.

129. Soveri I, Berg UB, Bjork J, et al. Measuring GFR: a systematic review. Am J Kidney Dis. 2014;64:411-424.

130. Ognibene A, Grandi G, Lorubbio M, et al. KDIGO 2012 clinical practice guideline CKD classification rules out creatinine clearance 24 hour urine collection? Clin Biochem. 2016;49:85-89.

131. Gaillard F, Flamant M, Lemoine S, et al. Estimated or measured GFR in living kidney donors work-up? Am J Transplant. 2016;16:3024-3032.
132. Fan L, Inker LA, Rossert J, et al. Glomerular filtration rate estimation using cystatin $\mathrm{C}$ alone or combined with creatinine as a confirmatory test. Nephrol Dial Transplant. 2014;29:1195-1203.

133. Inker LA, Schmid $\mathrm{CH}$, Tighiouart $\mathrm{H}$, et al. Estimating glomerular filtration rate from serum creatinine and cystatin C. N Engl J Med. 2012;367:20-29.

134. Matsushita K, van der Velde M, Astor BC, et al. Association of estimated glomerular filtration rate and albuminuria with all-cause and cardiovascular mortality in general population cohorts: a collaborative meta-analysis. Lancet. 2010;375:2073-2081.

135. Levey AS, de Jong PE, Coresh J, et al. The definition, classification, and prognosis of chronic kidney disease: a KDIGO Controversies Conference report. Kidney Int. 2011;80:17-28.

136. Matsushita K, Mahmoodi BK, Woodward M, et al. Comparison of risk prediction using the CKD-EPI equation and the MDRD study equation for estimated glomerular filtration rate. JAMA. 2012;307:1941-1951.

137. Shlipak MG, Matsushita K, Arnlov J, et al. Cystatin C versus creatinine in determining risk based on kidney function. N Engl J Med. 2013;369: 932-943.

138. Hallan SI, Matsushita K, Sang $\mathrm{Y}$, et al. Age and association of kidney measures with mortality and end-stage renal disease. JAMA. 2012; 308:2349-2360.

139. Kasiske BL, Anderson-Haag T, Israni AK, et al. A prospective controlled study of living kidney donors: three-year follow-up. Am J Kidney Dis. 2015:66:114-124.

140. Ibrahim HN, Foley R, Tan L, et al. Long-term consequences of kidney donation. N Engl J Med. 2009;360:459-469.

141. Garg AX, Muirhead N, Knoll G, et al. Proteinuria and reduced kidney function in living kidney donors: a systematic review, meta-analysis, and meta-regression. Kidney Int. 2006;70:1801-1810.

142. Cohney S, Kanellis J, Howell M. The CARI guidelines. Donor renal function. Nephrology (Carlton). 2010;15(Suppl 1):S137-S145.

143. Blantz RC, Steiner RW. Benign hyperfiltration after living kidney donation. J Clin Invest. 2015;125:972-974.

144. Lenihan CR, Busque S, Derby G, et al. Longitudinal study of living kidney donor glomerular dynamics after nephrectomy. J Clin Invest. 2015;125: 1311-1318.

145. Fehrman-Ekholm I, Duner F, Brink B, et al. No evidence of accelerated loss of kidney function in living kidney donors: results from a crosssectional follow-up. Transplantation. 2001;72:444-449.

146. Fournier C, Pallet N, Cherqaoui Z, et al. Very long-term follow-up of living kidney donors. Transp/ Int. 2012;25:385-390.

147. Cherikh WS, Young CJ, Kramer BF, et al. Ethnic and gender related differences in the risk of end-stage renal disease after living kidney donation. Am J Transplant. 2011;11:1650-1655.

148. LaPointe Rudow D, Hays R, Baliga P, et al. Consensus conference on best practices in live kidney donation: recommendations to optimize education, access, and care. Am J Transplant. 2015;15: 914-922.

149. Wang X, Vrtiska TJ, Avula RT, et al. Age, kidney function, and risk factors associate differently with cortical and medullary volumes of the kidney. Kidney Int. 2014:85:677-685.

150. Glodny B, Unterholzner V, Taferner B, et al. Normal kidney size and its influencing factors-a 64-slice MDCT study of 1.040 asymptomatic patients. BMC Urol. 2009;9:19.

151. Emamian SA, Nielsen MB, Pedersen JF, et al. Kidney dimensions at sonography: correlation with age, sex, and habitus in 665 adult volunteers. AJR Am J Roentgenol. 1993;160:83-86.

152. Norden $G$, Lennerling A, Nyberg G. Low absolute glomerular filtration rate in the living kidney donor: a risk factor for graft loss. Transplantation. 2000;70:1360-1362

153. Zaky ZS, Gebreselassie S, Poggio ED. Evaluation of kidney function and structure in potential living kidney donors: implications for the donor and recipient. Curr Transpl Rep. 2015;2:15-21.

154. Mandelbrot DA, Pavlakis M, Danovitch GM, et al. The medical evaluation of living kidney donors: a survey of US transplant centers. Am J Transplant. 2007; 7:2333-2343.

155. Inker LA. Albuminuria: time to focus on accuracy. Am J Kidney Dis. 2014;63:378-381

156. Gansevoort RT, Verhave JC, Hillege HL, et al. The validity of screening based on spot morning urine samples to detect subjects with microalbuminuria in the general population. Kidney Int Suppl. 2005: S28-35

157. Mogensen CL. Microalbuminuria and kidney function: notes on methods, interpretation and classification. NY: Wiley; 1986. 
158. Boudville N, Kanellis J. The CARI guidelines. Donors at risk: proteinuria. Nephrology (Carlton). 2010;15(Suppl 1):S106-S110.

159. Kasiske BL, Ravenscraft M, Ramos EL, et al. The evaluation of living renal transplant donors: clinical practice guidelines. Ad Hoc Clinical Practice Guidelines Subcommittee of the Patient Care and Education Committee of the American Society of Transplant Physicians. J Am Soc Nephrol. 1996;7:2288-2313.

160. Cohen RA, Brown RS. Clinical practice. Microscopic hematuria. $N$ Engl J Med. 2003;348:2330-2338.

161. Davis R, Jones JS, Barocas DA, et al. Diagnosis, evaluation and follow-up of asymptomatic microhematuria (AMH) in adults: AUA guideline. J Urol. 2012;188:2473-2481.

162. Sutton JM. Evaluation of hematuria in adults. JAMA. 1990;263:2475-2480.

163. Vivante A, Afek A, Frenkel-Nir Y, et al. Persistent asymptomatic isolated microscopic hematuria in Israeli adolescents and young adults and risk for end-stage renal disease. JAMA. 2011;306:729-736.

164. Chow KM, Kwan BC, Li PK, et al. Asymptomatic isolated microscopic haematuria: long-term follow-up. QJM. 2004;97:739-745.

165. Kovacevic Z, Jovanovic D, Rabrenovic V, et al. Asymptomatic microscopic haematuria in young males. Int J Clin Pract. 2008;62: 406-412.

166. Lee YM, Baek SY, Kim JH, et al. Analysis of renal biopsies performed in children with abnormal findings in urinary mass screening. Acta Paediatr. 2006:95:849-853.

167. Park YH, Choi JY, Chung HS, et al. Hematuria and proteinuria in a mass school urine screening test. Pediatr Nephrol. 2005;20:1126-1130.

168. Lam NN, Lentine KL, Garg AX, et al. Renal and cardiac assessment of living kidney donor candidates. Nat Rev Nephrol. 2017;13:420-428.

169. Savige J, Rana K, Tonna S, et al. Thin basement membrane nephropathy. Kidney Int. 2003;64:1169-1178.

170. Koushik R, Garvey C, Manivel JC, et al. Persistent, asymptomatic, microscopic hematuria in prospective kidney donors. Transplantation. 2005;80:1425-1429.

171. Choi SR, Sun IO, Hong YA, et al. The role of kidney biopsy to determine donation from prospective kidney donors with asymptomatic urinary abnormalities. Transplant Proc. 2012;44:11-13.

172. Jais JP, Knebelmann B, Giatras I, et al. X-linked Alport syndrome: natural history and genotype-phenotype correlations in girls and women belonging to 195 families: a "European Community Alport Syndrome Concerted Action" study. J Am Soc Nephrol. 2003;14:2603-2610.

173. Temme J, Peters F, Lange $\mathrm{K}$, et al. Incidence of renal failure and nephroprotection by RAAS inhibition in heterozygous carriers of X-chromosomal and autosomal recessive Alport mutations. Kidney Int. 2012;81:779-783.

174. Gross O, Weber M, Fries JW, et al. Living donor kidney transplantation from relatives with mild urinary abnormalities in Alport syndrome: long-term risk, benefit and outcome. Nephrol Dial Transplant. 2009; 24:1626-1630.

175. D'Amico G. Natural history of idiopathic IgA nephropathy and factors predictive of disease outcome. Semin Nephrol. 2004;24:179-196.

176. Szeto CC, Lai FM, To KF, et al. The natural history of immunoglobulin a nephropathy among patients with hematuria and minimal proteinuria. Am J Med. 2001;110:434-437.

177. Suzuki K, Honda K, Tanabe K, et al. Incidence of latent mesangial IgA deposition in renal allograft donors in Japan. Kidney Int. 2003;63: 2286-2294.

178. Kido R, Shibagaki Y, Iwadoh K, et al. Persistent glomerular hematuria in living kidney donors confers a risk of progressive kidney disease in donors after heminephrectomy. Am J Transplant. 2010;10:1597-1604

179. Richardson R, Connelly M, Dipchand C, et al. Kidney paired donation protocol for participating donors 2014. Transplantation. 2015;99:S1-S88.

180. Ierino $F$, Kanellis J, CARl. The CARl guidelines. Donors at risk: haematuria. Nephrology (Carlton). 2010;15(Suppl 1):S111-S113.

181. Savige J, Gregory M, Gross O, et al. Expert guidelines for the management of Alport syndrome and thin basement membrane nephropathy. J Am Soc Nephrol. 2013;24:364-375.

182. Lorenz EC, Lieske JC, Vrtiska TJ, et al. Clinical characteristics of potential kidney donors with asymptomatic kidney stones. Nephrol Dial Transplant. 2011;26:2695-2700.

183. Hughes P, Caring for Australians with Renal Impairment (CARI). The CARI guidelines. Kidney stones epidemiology. Nephrology (Carlton). 2007; 12(Suppl 1):S26-S30.

184. Thomas SM, Lam NN, Welk BK, et al. Risk of kidney stones with surgical intervention in living kidney donors. Am J Transplant. 2013; 13:2935-2944.
185. Olsburgh J, Thomas K, Wong K, et al. Incidental renal stones in potential live kidney donors: prevalence, assessment and donation, including role of ex vivo ureteroscopy. BJU Int. 2013;111:784-792.

186. Pearle MS, Goldfarb DS, Assimos DG, et al. Medical management of kidney stones: AUA guideline. J Urol. 2014;192:316-324.

187. Türk C, Petřík A, Sarica K, et al. EAU guidelines on diagnosis and conservative management of urolithiasis. Eur Urol. 2016;69:468-474.

188. Ferraro PM, Curhan GC, D'Addessi A, et al. Risk of recurrence of idiopathic calcium kidney stones: analysis of data from the literature. J Nephrol. 2017;30:227-233.

189. Rule AD, Krambeck AE, Lieske JC. Chronic kidney disease in kidney stone formers. Clin J Am Soc Nephrol. 2011;6:2069-2075.

190. Alexander RT, Hemmelgarn BR, Wiebe N, et al. Kidney stones and kidney function loss: a cohort study. BMJ. 2012;345:e5287.

191. Shoag J, Halpern J, Goldfarb DS, et al. Risk of chronic and end stage kidney disease in patients with nephrolithiasis. J Urol. 2014;192:1440-1445.

192. Kummer AE, Grams M, Lutsey P, et al. Nephrolithiasis as a risk factor for CKD: the Atherosclerosis Risk in Communities Study. Clin J Am Soc Nephrol. 2015;10:2023-2029.

193. Strang AM, Lockhart ME, Amling CL, et al. Living renal donor allograft lithiasis: a review of stone related morbidity in donors and recipients. J Urol. 2008;179:832-836.

194. Fink HA, Wilt TJ, Eidman KE, et al. Medical management to prevent recurrent nephrolithiasis in adults: a systematic review for an American College of Physicians Clinical Guideline. Ann Intern Med. 2013;158: 535-543.

195. Qaseem A, Dallas P, Forciea MA, et al. Dietary and pharmacologic management to prevent recurrent nephrolithiasis in adults: a clinical practice guideline from the American College of Physicians. Ann Intern Med. 2014;161:659-667.

196. AST/ASTS/NATCO/UNOS Joint Societies Work Group. Evaluation of the Living Kidney Donor-a Consensus Document from the AST/ ASTS/NATCO/UNOS Joint Societies Work Group.

197. Campion EW, Glynn RJ, DeLabry LO. Asymptomatic hyperuricemia. Risks and consequences in the Normative Aging Study. Am J Med. 1987:82:421-426.

198. Zhang W, Doherty M, Pascual E, et al. EULAR evidence based recommendations for gout. Part I: Diagnosis. Report of a task force of the Standing Committee for International Clinical Studies Including Therapeutics (ESCISIT). Ann Rheum Dis. 2006;65:1301-1311.

199. Lipkowitz MS. Regulation of uric acid excretion by the kidney. Curr Rheumatol Rep. 2012;14:179-188.

200. Krishnan E. Reduced glomerular function and prevalence of gout NHANES 2009-10. PLoS One. 2012;7:e50046.

201. Kasiske BL, Anderson-Haag T, Ibrahim HN, et al. A prospective controlled study of kidney donors: baseline and 6-month follow-up. Am J Kidney Dis. 2013;62:577-586.

202. Moody WE, Ferro CJ, Edwards NC, et al. Cardiovascular effects of unilateral nephrectomy in living kidney donors. Hypertension. 2016; 67:368-377.

203. Lam NN, McArthur E, Kim SJ, et al. Gout after living kidney donation: a matched cohort study. Am J Kidney Dis. 2015;65:925-932.

204. Lam NN, Garg AX, Segev DL, et al. Gout after living kidney donation: correlations with demographic traits and renal complications. Am J Nephrol. 2015;41:231-240.

205. Braga F, Pasqualetti S, Ferraro S, et al. Hyperuricemia as risk factor for coronary heart disease incidence and mortality in the general population: a systematic review and meta-analysis. Clin Chem Lab Med. 2016:54:7-15

206. Khanna D, Fitzgerald JD, Khanna PP, et al. 2012 American College of Rheumatology guidelines for management of gout. Part 1: systematic nonpharmacologic and pharmacologic therapeutic approaches to hyperuricemia. Arthritis Care Res (Hoboken). 2012;64:1431-1446.

207. van Echteld IA, van Durme C, Falzon L, et al. Treatment of gout patients with impairment of renal function: a systematic literature review. J Rheumatol Suppl. 2014;92:48-54.

208. Kasiske BL, Kumar R, Kimmel PL, et al. Abnormalities in biomarkers of mineral and bone metabolism in kidney donors. Kidney Int. 2016;90: 861-868.

209. Young A, Hodsman AB, Boudville N, et al. Bone and mineral metabolism and fibroblast growth factor 23 levels after kidney donation. Am J Kidney Dis. 2012;59:761-769.

210. Garg AX, Pouget J, Young A, et al. Fracture risk in living kidney donors: a matched cohort study. Am J Kidney Dis. 2012;59:770-776. 
211. Textor SC, Taler SJ, Larson TS, et al. Blood pressure evaluation among older living kidney donors. J Am Soc Nephrol. 2003;14:2159-2167.

212. O'Brien E, Parati G, Stergiou G, et al. European Society of Hypertension position paper on ambulatory blood pressure monitoring. $J$ Hypertens. 2013;31:1731-1768.

213. Chobanian AV, Bakris GL, Black HR, et al. Seventh report of the Joint National Committee on prevention, detection, evaluation, and treatment of high blood pressure. Hypertension. 2003;42:1206-1252.

214. lerino F, Boudville N, Kanellis J. The CARI guidelines. Donors at risk: hypertension. Nephrology (Carlton). 2010;15(Suppl 1):S114-S120.

215. Pascual J, Abramowicz D, Cochat P, et al. European renal best practice guideline on the management and evaluation of the kidney donor and recipient. Nefrologia. 2014;34:293-301.

216. Stergiou GS, Asayama K, Thijs L, et al. Prognosis of white-coat and masked hypertension: International Database of HOme blood pressure in relation to Cardiovascular Outcome. Hypertension. 2014;63: 675-682.

217. Hanninen MR, Niranen TJ, Puukka PJ, et al. Target organ damage and masked hypertension in the general population: the Finn-Home study. $J$ Hypertens. 2013;31:1136-1143.

218. Aljadhey H, Tu W, Hansen RA, et al. Comparative effects of nonsteroidal anti-inflammatory drugs (NSAIDs) on blood pressure in patients with hypertension. BMC Cardiovasc Disord. 2012;12:93.

219. Elliott WJ. Drug interactions and drugs that affect blood pressure. J Clin Hypertens (Greenwich). 2006;8:731-737.

220. Snowden S, Nelson R. The effects of nonsteroidal anti-inflammatory drugs on blood pressure in hypertensive patients. Cardiol Rev. 2011; 19:184-191.

221. Eckel RH, Jakicic JM, Ard JD, et al. 2013 AHAVACC guideline on lifestyle management to reduce cardiovascular risk: a report of the American College of Cardiology/American Heart Association Task Force on Practice Guidelines. J Am Coll Cardiol. 2014;63:2960-2984.

222. Young JH, Klag MJ, Muntner P, et al. Blood pressure and decline in kidney function: findings from the Systolic Hypertension in the Elderly Program (SHEP). J Am Soc Nephrol. 2002;13:2776-2782.

223. Vasan RS, Beiser A, Seshadri S, et al. Residual lifetime risk for developing hypertension in middle-aged women and men: the Framingham Heart Study. JAMA. 2002;287:1003-1010.

224. Boudville N, Prasad GV, Knoll G, et al. Meta-analysis: risk for hypertension in living kidney donors. Ann Intern Med. 2006;145:185-196.

225. Garg AX, Prasad GV, Thiessen-Philbrook HR, et al. Cardiovascular disease and hypertension risk in living kidney donors: an analysis of health administrative data in Ontario, Canada. Transplantation. 2008; 86:399-406.

226. Lentine KL, Schnitzler MA, Garg AX, et al. Understanding antihypertensive medication use after living kidney donation through linked national registry and pharmacy claims data. Am J Nephrol. 2014;40: 174-183.

227. Lentine KL, Schnitzler MA, Xiao H, et al. Consistency of racial variation in medical outcomes among publicly and privately insured living kidney donors. Transplantation. 2014;97:316-324.

228. Lentine $\mathrm{KL}$, Schnitzler MA, Xiao H, et al. Racial variation in medical outcomes among living kidney donors. N Engl J Med. 2010;363:724-732.

229. Doshi MD, Goggins MO, Li L, et al. Medical outcomes in African American live kidney donors: a matched cohort study. Am J Transplant. 2013;13:111-118.

230. LV J, Ehteshami P, Sarnak MJ, et al. Effects of intensive blood pressure lowering on the progression of chronic kidney disease: a systematic review and meta-analysis. CMAJ. 2013;185:949-957.

231. Lewis EJ, Hunsicker LG, Bain RP, et al. The effect of angiotensinconverting-enzyme inhibition on diabetic nephropathy. The Collaborative Study Group. N Engl J Med. 1993;329:1456-1462.

232. Brenner BM, Cooper ME, de Zeeuw D, et al. Effects of losartan on renal and cardiovascular outcomes in patients with type 2 diabetes and nephropathy. N Engl J Med. 2001;345:861-869.

233. Appel LJ, Wright JT Jr, Greene T, et al. Long-term effects of reninangiotensin system-blocking therapy and a low blood pressure goal on progression of hypertensive chronic kidney disease in African Americans. Arch Intern Med. 2008;168:832-839.

234. Appel LJ, Wright JT Jr, Greene T, et al. Intensive blood-pressure control in hypertensive chronic kidney disease. N Engl J Med. 2010;363:918-929.

235. Friedman DJ, Kozlitina J, Genovese G, et al. Population-based risk assessment of APOL1 on renal disease. J Am Soc Nephrol. 2011;22: 2098-2105.
236. Kanij Z, Powe CE, Wenger JB, et al. Genetic variation in APOL1 associates with younger age at hemodialysis initiation. J Am Soc Nephrol. 2011;22:2091-2097.

237. Kopp JB, Nelson GW, Sampath K, et al. APOL1 genetic variants in focal segmental glomerulosclerosis and HIV-associated nephropathy. J Am Soc Nephrol. 2011;22:2129-2137.

238. Parsa A, Kao WH, Xie D, et al. APOL1 risk variants, race, and progression of chronic kidney disease. N Engl J Med. 2013;369:2183-2196.

239. Meguid El Nahas A, Bello AK. Chronic kidney disease: the global challenge. Lancet. 2005;365:331-340.

240. Poggio ED, Braun WE, Davis C. The science of stewardship: due diligence for kidney donors and kidney function in living kidney donation —evaluation, determinants, and implications for outcomes. Clin J Am Soc Nephrol. 2009;4:1677-1684.

241. Oh CK, Jeon KO, Kim HJ, et al. Metabolic demand and renal mass supply affecting the early graft function after living donor kidney transplantation. Kidney Int. 2005;67:744-749.

242. Rook M, Bosma RJ, van Son WJ, et al. Nephrectomy elicits impact of age and $\mathrm{BMI}$ on renal hemodynamics: lower postdonation reserve capacity in older or overweight kidney donors. Am J Transplant. 2008; 8:2077-2085.

243. Lee JH, Kim SC, Han DJ, et al. Risk factors for MDRD-GFR of less than $60 \mathrm{~mL} / \mathrm{min}$ per $1.73 \mathrm{~m} 2$ in former kidney donors. Nephrology (Carlton). 2007;12:600-606.

244. Gracida C, Espinoza R, Cedillo U, et al. Kidney transplantation with living donors: nine years of follow-up of 628 living donors. Transplant Proc. 2003;35:946-947

245. Textor SC, Taler SJ, Driscoll N, et al. Blood pressure and renal function after kidney donation from hypertensive living donors. Transplantation. 2004;78:276-282.

246. World Health Organization (WHO). Global Report on Diabetes. Executive Summary. Available at: http://apps.who.int/iris/bitstream/10665/ 204874/1MHO_NMH_NVI_16.3_eng.pdf. (Accessed: July 31, 2016).

247. Ng M, Fleming T, Robinson $\mathrm{M}$, et al. Global, regional, and national prevalence of overweight and obesity in children and adults during 1980-2013: a systematic analysis for the Global Burden of Disease Study 2013. Lancet. 2014;384:766-781.

248. World Health Organization. Obesity: Preventing and managing global epidemic. WHO Technical Report Series No 894, 2000.

249. Padwal RS. Obesity, diabetes, and the metabolic syndrome: the global scourge. Can J Cardiol. 2014;30:467-472.

250. Zobel EH, Hansen TW, Rossing P, et al. Global changes in food supply and the obesity epidemic. Curr Obes Rep. 2016;5:449-455.

251. World Health Organization. Waist Circumference and Waist-Hip Ratio. Report of a WHO Expert Consultation. Geneva, 8-11 2008.

252. Boudville N, Isbel N. The CARI guidelines. Donors at risk: impaired glucose tolerance. Nephrology (Carlton). 2010;15(Suppl 1):S133-S136.

253. World Health Organization. Global Report on Diabetes. 2016.

254. Makaroff LE. The need for international consensus on prediabetes. Lancet Diabetes Endocrinol. 2017;5:5-7.

255. Stone NJ, Robinson JG, Lichtenstein AH, et al. 2013 ACC/AHA guideline on the treatment of blood cholesterol to reduce atherosclerotic cardiovascular risk in adults: a report of the American College of Cardiology/American Heart Association Task Force on Practice Guidelines. J Am Coll Cardiol. 2014;63:2889-2934.

256. DeMaria EJ, Carmody BJ. Perioperative management of special populations: obesity. Surg Clin North Am. 2005;85:1283-1289.

257. D'Agati VD, Chagnac A, de Vries AP, et al. Obesity-related glomerulopathy: clinical and pathologic characteristics and pathogenesis. Nat Rev Nephrol. 2016;12:453-471.

258. von Zur-Muhlen B, Berglund D, Yamamoto S, et al. Single-centre longterm follow-up of live kidney donors demonstrates preserved kidney function but the necessity of a structured lifelong follow-up. Ups J Med Sci. 2014;119:236-241.

259. Isbel N, CARI guidelines. The CARI guidelines. Donors at risk: obesity. Nephrology (Carlton). 2010;15(Suppl 1):S121-S132.

260. Locke JE, Reed RD, Massie A, et al. Obesity increases the risk of end-stage renal disease among living kidney donors. Kidney Int. 2017; 91:699-703.

261. Narayan KM, Boyle JP, Thompson TJ, et al. Lifetime risk for diabetes mellitus in the United States. JAMA. 2003;290:1884-1890.

262. Plantinga LC, Crews DC, Coresh J, et al. Prevalence of chronic kidney disease in US adults with undiagnosed diabetes or prediabetes. Clin J Am Soc Nephrol. 2010;5:673-682. 
263. Okamoto M, Suzuki T, Fujiki M, et al. The consequences for live kidney donors with preexisting glucose intolerance without diabetic complication: analysis at a single Japanese center. Transplantation. 2010;89: 1391-1395.

264. Tabak AG, Herder C, Rathmann W, et al. Prediabetes: a high-risk state for diabetes development. Lancet. 2012;379:2279-2290.

265. Ferrannini E. Definition of intervention points in prediabetes. Lancet Diabetes Endocrinol. 2014;2:667-675.

266. Ligthart S, van Herpt TT, Leening MJ, et al. Lifetime risk of developing impaired glucose metabolism and eventual progression from prediabetes to type 2 diabetes: a prospective cohort study. Lancet Diabetes Endocrinol. 2016;4:44-51.

267. Chandran S, Masharani U, Webber AB, et al. Prediabetic living kidney donors have preserved kidney function at 10 years after donation. Transplantation. 2014;97:748-754.

268. Zammit AR, Katz MJ, Derby C, et al. Metabolic syndrome and smoking are associated with future development of advanced chronic kidney disease in older adults. Cardiorenal Med. 2016;6:108-115.

269. Young BA, Katz R, Boulware LE, et al. Risk factors for rapid kidney function decline among African Americans: the Jackson Heart Study (JHS). Am J Kidney Dis. 2016;68:229-239.

270. Garg AX, Meirambayeva A, Huang A, et al. Cardiovascular disease in kidney donors: matched cohort study. BMJ. 2012;344:e1203.

271. Kaplan B, llahe A. Quantifying risk of kidney donation: the truth is not out there (yet). Am J Transplant. 2014;14:1715-1716.

272. Amer H, Prieto M, Heimbach JK, et al. Increasing mortality by living kidney donation? The devil is in the details. Kidney Int. 2014;85:1469.

273. Kirchner VA, Liu PT, Pruett TL. Infection and cancer screening in potential living donors: best practices to protect the donor and recipient. Curr Transpl Rep. 2015;2:35-43.

274. Fishman JA, Grossi PA. Donor-derived infection-the challenge for transplant safety. Nat Rev Nephrol. 2014;10:663-672.

275. Len O, Garzoni C, Lumbreras C, et al. Recommendations for screening of donor and recipient prior to solid organ transplantation and to minimize transmission of donor-derived infections. Clin Microbiol Infect. 2014;20(Suppl 7):10-18.

276. Morris MI, Fischer SA, Ison MG. Infections transmitted by transplantation. Infect Dis Clin North Am. 2010;24:497-514.

277. Bohl DL, Brennan DC, Ryschkewitsch C, et al. BK virus antibody titers and intensity of infections after renal transplantation. J Clin Virol. 2008; 43:184-189.

278. Bohl DL, Storch GA, Ryschkewitsch C, et al. Donor origin of BK virus in renal transplantation and role of $\mathrm{HLA} \mathrm{C7}$ in susceptibility to sustained BK viremia. Am J Transplant. 2005;5:2213-2221.

279. Mwintshi K, Brennan DC. Prevention and management of cytomegalovirus infection in solid-organ transplantation. Expert Rev Anti Infect Ther. 2007;5:295-304.

280. Andrews PA, Emery VC, Newstead C. Summary of the British Transplantation Society guidelines for the prevention and management of CMV disease after solid organ transplantation. Transplantation. 2011;92: $1181-1187$.

281. Brennan DC, Agha I, Bohl DL, et al. Incidence of BK with tacrolimus versus cyclosporine and impact of preemptive immunosuppression reduction. Am J Transplant. 2005;5:582-594.

282. Spinner ML, Saab G, Casabar E, et al. Impact of prophylactic versus preemptive valganciclovir on long-term renal allograft outcomes. Transplantation. 2010;90:412-418.

283. Ison MG, Grossi P, AST Infectious Diseases Community of Practice. Donor-derived infections in solid organ transplantation. Am J Transplant. 2013;13(Suppl 4):22-30.

284. HIV transmitted from a living organ donor-New York City, 2009. Am J Transplant. 2011;11:1334-1337.

285. Garzoni C, Ison MG. Uniform definitions for donor-derived infectious disease transmissions in solid organ transplantation. Transplantation. 2011; 92:1297-1300.

286. Levi ME, Kumar D, Green M, et al. Considerations for screening live kidney donors for endemic infections: a viewpoint on the UNOS policy. Am J Transplant. 2014;14:1003-1011.

287. OPTN (Organ Procurement and Transplantation Network)/UNOS (United Network for Organ Sharing). Recognizing Seasonal and Geographically Endemic Infections in Organ Donors: Considerations during Living Donor Evaluation. 2014 (http://optn.transplant.hrsa.gov/media/1138/seasonal_disease_guidance.pdf). (Accessed: September 7, 2016).
288. OPTN (Organ Procurement and Transplantation Network)/UNOS (United Network for Organ Sharing) Ad Hoc Disease Transmission Advisory Committee (DTAC). Guidance for Identifying Risk Factors for Mycobacterium tuberculosis (MTB) During Evaluation of Potential Living Kidney Donors. Available at: https://optn.transplant.hrsa.gov/resources/ guidance/guidance-for-identifying-risk-factors-for-mycobacteriumtuberculosis-mtb-during-evaluation-of-potential-living-kidney-donors/. (Accessed September 7, 2016).

289. Chin-Hong PV, Schwartz BS, Bern C, et al. Screening and treatment of Chagas disease in organ transplant recipients in the United States: recommendations from the Chagas in transplant working group. Am J Transplant. 2011;11:672-680.

290. Morris MI, Daly JS, Blumberg E, et al. Diagnosis and management of tuberculosis in transplant donors: a donor-derived infections consensus conference report. Am J Transplant. 2012;12:2288-2300.

291. OPTN (Organ Procurement and Transplantation Network)/UNOS (United Network for Organ Sharing) Ad Hoc Disease Transmission Advisory Committee (DTAC). Identifying Risk Factors for West Nile Virus (WNV During Evaluation of Potential Living Donors. Available at: https://optn. transplant.hrsa.gov/resources/guidance/identifying-risk-factorsfor-west-nile-virus-wnv-during-evaluation-of-potential-living-donors/. (Accessed September 7, 2016).

292. Feng S, Buell JF, Cherikh WS, et al. Organ donors with positive viral serology or malignancy: risk of disease transmission by transplantation. Transplantation. 2002;74:1657-1663.

293. Ouseph R, Eng M, Ravindra K, et al. Review of the use of hepatitis B core antibody-positive kidney donors. Transplant Rev (Orlando). 2010;24: 167-171.

294. Centers for Disease Control and Prevention (CDC). Testing recommendations for hepatitis C virus infection. Available at: http://www.cdc. gov/hepatitis/hcv/guidelinesc.htm. (Accessed: September 7, 2016).

295. Johnson RJ, Gretch DR, Yamabe H, et al. Membranoproliferative glomerulonephritis associated with hepatitis $\mathrm{C}$ virus infection. $N$ Engl $J$ Med. 1993;328:465-470.

296. Natov SN. Transmission of viral hepatitis by kidney transplantation: donor evaluation and transplant policies (Part 2: hepatitis C virus). Transpl Infect Dis. 2002;4:124-131.

297. Abbott KC, Lentine KL, Bucci JR, et al. The impact of transplantation with deceased donor hepatitis C-positive kidneys on survival in waitlisted long-term dialysis patients. Am J Transplant. 2004;4:2032-2037.

298. European Association for the Study of the Liver. EASL recommendations on treatment of hepatitis C 2016. J Hepatol 2017;66:153-194.

299. Busch MP, Glynn SA, Stramer SL, et al. A new strategy for estimating risks of transfusion-transmitted viral infections based on rates of detection of recently infected donors. Transfusion. 2005;45:254-264.

300. Muller E, Kahn D, Mendelson M. Renal transplantation between HIVpositive donors and recipients. N Engl J Med. 2010;362:2336-2337.

301. U.S. Federal Register. Human Immunodeficiency Virus (HIV) Organ Policy Equity (HOPE) Act Safeguards and Research Criteria for Transplantation of Organs Infected With HIV. A Notice by the National Institutes of Health on 06/18/2015. Available at: https://www.federalregister.gov/ articles/2015/06/18/2015-15034/human-immunodeficiency-virushiv-organ-policy-equity-hope-act-safeguards-and-research-criteria-for. (Accessed September 7, 2016).

302. Ison MG, Llata E, Conover CS, et al. Transmission of human immunodeficiency virus and hepatitis $C$ virus from an organ donor to four transplant recipients. Am J Transplant. 2011;11:1218-1225.

303. Blumberg EA, Ison MG, Pruett TL, et al. Optimal testing of the live organ donor for blood-borne viral pathogens: the report of a consensus conference. Am J Transplant. 2013;13:1405-1415.

304. Ison MG, Abecassis M, Blumberg E, et al. Achieving consensus on increased risk donors to improve access to organ transplantation. Northwestern Agency for Healthcare Research and Quality-funded Consensus Conference, presented to the Advisory Committee on Organ Transplantation of the U.S. Department of Health and Human Services, August 2, 2012. Available at: http://www.feinberg.northwestern.edu/sites/transplant/ docs/conference/FinalReport.pdf. (Accessed September 7, 2016).

305. Echenique IA, Cohen D, Rudow DL, et al. Impact of repeat testing of living kidney donors within 14 days of the transplant procedure: a multicenter retrospective survey. Transp/ Infect Dis. 2014;16:403-411.

306. Brennan DC, Aguado JM, Potena L, et al. Effect of maintenance immunosuppressive drugs on virus pathobiology: evidence and potential mechanisms. Rev Med Virol. 2013;23:97-125.

307. Green M, Michaels MG. Epstein-Barr virus infection and posttransplant lymphoproliferative disorder. Am J Transplant. 2013;13(Suppl 3):41-54. 
308. Morton M, Coupes B, Roberts SA, et al. Epstein-Barr virus infection in adult renal transplant recipients. Am J Transplant. 2014;14:1619-1629.

309. Beam E, Razonable RR. Cytomegalovirus in solid organ transplantation: epidemiology, prevention, and treatment. Curr Infect Dis Rep. 2012;14:633-641.

310. Preiksaitis JK, Brennan DC, Fishman J, et al. Canadian Society of Transplantation consensus workshop on cytomegalovirus management in solid organ transplantation final report. Am J Transplant. 2005; 5:218-227.

311. Hodson EM, Ladhani M, Webster AC, et al. Antiviral medications for preventing cytomegalovirus disease in solid organ transplant recipients. Cochrane Database Syst Rev. 2013;2:CD003774.

312. Cortes NJ, Afzali B, MacLean D, et al. Transmission of syphilis by solid organ transplantation. Am J Transplant. 2006;6:2497-2499.

313. Cortez KJ, Greenwald MA. Current trends in donor testing to detect syphilis infection. Curr Infect Dis Rep. 2014;16:423.

314. Theodoropoulos N, Jaramillo A, Penugonda S, et al. Improving syphilis screening in deceased organ donors. Transplantation. 2015;99:438-443.

315. Meije Y, Piersimoni C, Torre-Cisneros J, et al. Mycobacterial infections in solid organ transplant recipients. Clin Microbiol Infect. 2014;20(Suppl 7): 89-101.

316. Ison MG, Nalesnik MA. An update on donor-derived disease transmission in organ transplantation. Am J Transplant. 2011;11:1123-1130.

317. Aguado JM, Torre-Cisneros J, Fortún J, et al. Tuberculosis in solid-organ transplant recipients: consensus statement of the group for the study of infection in transplant recipients (GESITRA) of the Spanish Society of Infectious Diseases and Clinical Microbiology. Clin Infect Dis. 2009; 48:1276-1284.

318. Subramanian AK. Tuberculosis in solid organ transplant candidates and recipients: current and future challenges. Curr Opin Infect Dis. 2014; 27:316-321.

319. Moon SM, Park IA, Kim SM, et al. Living donor and recipient screening for latent tuberculosis infection by tuberculin skin test and interferongamma releasing assay in a country with an intermediate burden of tuberculosis. J Infect Chemother. 2013;19:1009-1013.

320. Ariza-Heredia EJ, Beam EN, Lesnick TG, et al. Impact of urinary tract infection on allograft function after kidney transplantation. Clin Transplant. 2014;28:683-690.

321. Lee JR, Bang H, Dadhania $\mathrm{D}$, et al. Independent risk factors for urinary tract infection and for subsequent bacteremia or acute cellular rejection: a single-center report of 1166 kidney allograft recipients. Transplantation. 2013:96:732-738.

322. Abdalhamid BA, Al Abadi AN, Al Saghier Ml, et al. Strongyloides stercoralis infection in kidney transplant recipients. Saudi J Kidney Dis Transpl. 2015;26:98-102.

323. Le M, Ravin K, Hasan A, et al. Single donor-derived strongyloidiasis in three solid organ transplant recipients: case series and review of the literature. Am J Transplant. 2014;14:1199-1206.

324. Roseman DA, Kabbani D, Kwah J, et al. Strongyloides stercoralis transmission by kidney transplantation in two recipients from a common donor. Am J Transplant. 2013;13:2483-2486.

325. World Health organization (WHO). Strongyloidiasis Epidemiology. Available at: http://www.who.int/intestinal_worms/epidemiology/ strongyloidiasis/en/. (Accessed: September 7, 2016).

326. Huprikar S, Bosserman E, Patel G, et al. Donor-derived Trypanosoma cruzi infection in solid organ recipients in the United States, 2001-2011. Am J Transplant. 2013;13:2418-2425.

327. OPTN (Organ Procurement and Transplantation Network)/UNOS (United Network for Organ Sharing) Ad Hoc Disease Transmission Advisory Committee (DTAC). Guidance regarding Ebola virus disease. Available at: https://optn.transplant.hrsa.gov/news/guidance-regarding-ebolavirus-disease/. (Accessed: September 7, 2016).

328. World Health organization (WHO). Zika virus fact sheet (Updated 2 June 2016). Available at: http://www.who.int/mediacentre/factsheets/zika/ en/. (Accessed: September 7, 2016).

329. OPTN (Organ Procurement and Transplantation Network)/UNOS (United Network for Organ Sharing) Ad Hoc Disease Transmission Advisory Committee (DTAC). Guidance on Zika virus. Available at: https://optn.transplant. hrsa.gov/news/guidance-on-zika-virus/. (Accessed: September 7, 2016).

330. Centers for Disease Control and Prevention (CDC). Global Health. Available at: http://www.cdc.gov/globalhealth/index.html. (Accessed: September 7, 2016).

331. US Preventative Services Task Force. USPSTF Published recommendations for cancer screening. Available at: http://www.uspreventiveservicestaskforce. org/BrowseRec/Index. (Accessed: September 7, 2016).
332. American Cancer Society. Guidelines for the Early Detection of Cancer. Available at: http://www.cancer.org/healthy/findcancerearly/ cancerscreeningguidelines/american-cancer-society-guidelines-for-theearly-detection-of-cancer. (Accessed: September 7, 2016).

333. United Kindgom (UK) National Screening Committee (UK NSC). Current UK NSC recommendations. Available at: http://legacy.screening.nhs.uk/ screening-recommendations.php. (Accessed: September 7, 2016).

334. Lentine KL, Vijayan A, Xiao H, et al. Cancer diagnoses after living kidney donation: linking U.S. Registry data and administrative claims. Transplantation. 2012;94:139-144.

335. Xiao D, Craig JC, Chapman JR, et al. Donor cancer transmission in kidney transplantation: a systematic review. Am J Transplant. 2013;13:2645-2652.

336. Kauffman HM, Cherikh WS, McBride MA, et al. Deceased donors with a past history of malignancy: an organ procurement and transplantation network/united network for organ sharing update. Transplantation. 2007;84:272-274.

337. Strauss DC, Thomas JM. Transmission of donor melanoma by organ transplantation. Lancet Oncol. 2010;11:790-796.

338. Penn I. Malignant melanoma in organ allograft recipients. Transplantation. 1996;61:274-278.

339. Buell JF, Beebe TM, Trofe J, et al. Donor transmitted malignancies. Ann Transplant. 2004;9:53-56.

340. Bibbins-Domingo K, Grossman DC, Curry SJ, et al. Screening for skin cancer: US preventive services task force recommendation statement. JAMA. 2016:316:429-435.

341. Nalesnik MA, Woodle ES, Dimaio JM, et al. Donor-transmitted malignancies in organ transplantation: assessment of clinical risk. Am J Transplant. 2011;11:1140-1147.

342. Ordon M, Welk B, McArthur E, et al. Risk of nephrectomy in previous living kidney donors. Transplantation. 2016;100:1313-1317.

343. Israel GM, Bosniak MA. An update of the Bosniak renal cyst classification system. Urology. 2005;66:484-488.

344. Campbell SC, Lane BR. Chapter 57: Malignant renal tumors. In: Campbell-Walsh Urology, 11th Edn. Wein AJ, Kavoussi LR, Partin AW, et al. (eds). Elsevier, p. 1314-1364, 2016.

345. Musquera M, Pérez M, Peri L, et al. Kidneys from donors with incidental renal tumors: should they be considered acceptable option for transplantation? Transplantation. 2013;95:1129-1133.

346. Valente M, Furian L, Rigotti P. Organ donors with small renal cancer: report of 3 cases. Transplant Proc. 2012;44:1846-1847.

347. Sener A, Uberoi V, Bartlett ST, et al. Living-donor renal transplantation of grafts with incidental renal masses after ex-vivo partial nephrectomy. BJU Int. 2009;104:1655-1660.

348. Ghafari A. Transplantation of a kidney with a renal cell carcinoma after living donation: a case report. Transplant Proc. 2007;39:1660-1661.

349. Notify Library. The global vigilence and surveillance database for medical products of human origin. Available at: http://www.notifylibrary.org/. (Accessed: September 7, 2016).

350. Kuppachi S, Smith RJH, Thomas CP. Evaluation of genetic renal diseases in potential living kidney donors. Curr Transp/ Rep. 2015;2:1-14.

351. Chapman AB, Devuyst O, Eckardt KU, et al. Autosomal-dominant polycystic kidney disease (ADPKD): executive summary from a Kidney Disease: Improving Global Outcomes (KDIGO) Controversies Conference. Kidney Int. 2015;88:17-27.

352. Pei $\mathrm{Y}, \mathrm{Hwang} \mathrm{YH}$, Conklin J, et al. Imaging-based diagnosis of autosomal dominant polycystic kidney disease. J Am Soc Nephrol. 2015;26:746-753.

353. Kanaan N, Devuyst O, Pirson Y. Renal transplantation in autosomal dominant polycystic kidney disease. Nat Rev Nephrol. 2014;10:455-465.

354. Pei Y, Obaji J, Dupuis A, et al. Unified criteria for ultrasonographic diagnosis of ADPKD. J Am Soc Nephrol. 2009;20:205-212.

355. Simms RJ, Travis DL, Durkie M, et al. Genetic testing in the assessment of living related kidney donors at risk of autosomal dominant polycystic kidney disease. Transplantation. 2015;99:1023-1029.

356. Audrezet MP, Cornec-Le Gall E, Chen JM, et al. Autosomal dominant polycystic kidney disease: comprehensive mutation analysis of PKD1 and PKD2 in 700 unrelated patients. Hum Mutat. 2012;33:1239-1250.

357. Rossetti S, Consugar MB, Chapman AB, et al. Comprehensive molecular diagnostics in autosomal dominant polycystic kidney disease. J Am Soc Nephrol. 2007;18:2143-2160.

358. Riella LV, Sheridan AM. Testing for high-risk APOL1 alleles in potential living kidney donors. Am J Kidney Dis. 2015;66:396-401.

359. Zwang NA, Shetty A, Sustento-Reodica N, et al. APOL1-associated end-stage renal disease in a living kidney transplant donor. Am J Transplant. 2016;16:3568-3572. 
360. Reeves-Daniel AM, DePalma JA, Bleyer AJ, et al. The APOL1 gene and allograft survival after kidney transplantation. Am J Transplant. 2011;11: 1025-1030.

361. Freedman BI, Pastan SO, Israni AK, et al. APOL1 genotype and kidney transplantation outcomes from deceased African American donors. Transplantation. 2016;100:194-202.

362. Kofman T, Audard V, Narjoz C, et al. APOL1 polymorphisms and development of CKD in an identical twin donor and recipient pair. Am J Kidney Dis. 2014;63:816-819.

363. Cohen DM, Mittalhenkle A, Scott DL, et al. African American living-kidney donors should be screened for APOL1 risk alleles. Transplantation. 2011;92:722-725.

364. Lentine KL, Segev DL. Health outcomes among non-Caucasian living kidney donors: knowns and unknowns. Transpl Int. 2013;26:853-864.

365. Ross LF, Thistlethwaite JR Jr. Introducing genetic tests with uncertain implications in living donor kidney transplantation: ApoL1 as a case study. Prog Transplant. 2016;26:203-206.

366. Chandraker $A$. The real world impact of APOL1 variants on kidney transplantation. Transplantation. 2016;100:16-17.

367. Freedman BI, Julian BA. Should kidney donors be genotyped for APOL1 risk alleles? Kidney Int. 2015;87:671-673.

368. Lentine KL, Schnitzler MA, Garg AX, et al. Race, relationship and renal diagnoses after living kidney donation. Transplantation. 2015;99:1723-1729.

369. Newell KA, Formica RN, Gill JS, et al. Integrating APOL-1 gene variants into renal transplantation: considerations arising from the American Society of Transplantation expert conference. Am J Transplant. 2017;17:901-911.

370. Chan MM, Gale DP. Isolated microscopic haematuria of glomerular origin: clinical significance and diagnosis in the 21st century. Clin Med (Lond). 2015;15:576-580.

371. Miner JH, Baigent C, Flinter F, et al. The 2014 international workshop on Alport syndrome. Kidney Int. 2014;86:679-684.

372. Niaudet P. Living donor kidney transplantation in patients with hereditary nephropathies. Nat Rev Nephrol. 2010;6:736-743.

373. Heidet L, Gubler MC. The renal lesions of Alport syndrome. J Am Soc Nephrol. 2009;20:1210-1215.

374. Gast C, Pengelly RJ, Lyon M, et al. Collagen (COL4A) mutations are the most frequent mutations underlying adult focal segmental glomerulosclerosis. Nephrol Dial Transplant. 2016;31:961-970.

375. Weber S, Strasser K, Rath S, et al. Identification of 47 novel mutations in patients with Alport syndrome and thin basement membrane nephropathy. Pediatr Nephrol. 2016;31:941-955.

376. Kashtan CE. Women with Alport syndrome: risks and rewards of kidney donation. Nephrol Dial Transplant. 2009;24:1369-1370.

377. Favalli V, Disabella E, Molinaro M, et al. Genetic screening of AndersonFabry disease in probands referred from multispecialty clinics. J Am Coll Cardiol. 2016;68:1037-1050.

378. Popli S, Molnar ZV, Leehey DJ, et al. Involvement of renal allograft by Fabry's disease. Am J Nephrol. 1987;7:316-318.

379. Rood IM, Deegens JK, Wetzels JF. Genetic causes of focal segmental glomerulosclerosis: implications for clinical practice. Nephrol Dial Transplant. 2012;27:882-890.

380. Pollak MR. Familial FSGS. Adv Chronic Kidney Dis. 2014;21:422-425.

381. Sadowski CE, Lovric S, Ashraf S, et al. A single-gene cause in $29.5 \%$ of cases of steroid-resistant nephrotic syndrome. J Am Soc Nephrol. 2015; 26:1279-1289.

382. Winn MP, Alkhunaizi AM, Bennett WM, et al. Focal segmental glomerulosclerosis: a need for caution in live-related renal transplantation. Am J Kidney Dis. 1999;33:970-974.

383. Donne RL, Abbs I, Barany P, et al. Recurrence of hemolytic uremic syndrome after live related renal transplantation associated with subsequent de novo disease in the donor. Am J Kidney Dis. 2002;40:E22.

384. Zuber J, Fakhouri F, Roumenina LT, et al. Use of eculizumab for atypical haemolytic uraemic syndrome and C3 glomerulopathies. Nat Rev Nephrol. 2012;8:643-657.

385. Hart TC, Gorry MC, Hart PS, et al. Mutations of the UMOD gene are responsible for medullary cystic kidney disease 2 and familial juvenile hyperuricaemic nephropathy. J Med Genet. 2002;39:882-892.

386. Kirby A, Gnirke A, Jaffe DB, et al. Mutations causing medullary cystic kidney disease type 1 lie in a large VNTR in MUC1 missed by massively parallel sequencing. Nat Genet. 2013;45:299-303.

387. Ojodu J, Hulihan MM, Pope SN, et al. Incidence of sickle cell traitUnited States, 2010. MMWR Morb Mortal Wkly Rep. 2014;63: 1155-1158.
388. Nagar R, Sinha S, Raman R. Haemoglobinopathies in eastern Indian states: a demographic evaluation. J Community Genet. 2015;6:1-8.

389. Key NS, Derebail VK. Sickle-cell trait: novel clinical significance. Hemato/ogy Am Soc Hematol Educ Program. 2010;2010:418-422.

390. Gebreselassie S, Simmons MN, Montague DK. Genitourinary manifestations of sickle cell disease. Cleve Clin J Med. 2015;82:679-683.

391. Reese PP, Hoo AC, Magee CC. Screening for sickle trait among potential live kidney donors: policies and practices in US transplant centers. Transpl Int. 2008;21:328-331.

392. Thomas CP, Mansilla MA, Sompallae R, et al. Screening of living kidney donors for genetic diseases using a comprehensive genetic testing strategy. Am J Transplant. 2017;17:401-410.

393. Department of Health and Human Services. APOL1 Long-term Kidney Transplantation Outcomes Network (APOLLO) Clinical Centers (Collaborative U01). https://grants. nih.gov/grants/guide/rfa-files/RFA-DK-16025.html. Accessed January 24, 2017.

394. Department of Health and Human Services. APOL 1 Long-term Kidney Transplantation Outcomes Network Scientific and Data Research Center (APOLLO SDRC) (Collaborative U01). https://grants.nih.gov/grants/ guide/rfa-files/RFA-DK-16-024.html. Accessed January 24, 2017.

395. Vikse BE. Pre-eclampsia and the risk of kidney disease. Lancet. 2013; 382:104-106.

396. McDonald SD, Han Z, Walsh MW, et al. Kidney disease after preeclampsia: a systematic review and meta-analysis. Am J Kidney Dis. 2010;55:1026-1039.

397. Vikse BE, Irgens LM, Leivestad T, et al. Preeclampsia and the risk of end-stage renal disease. N Engl J Med. 2008;359:800-809.

398. Wang IK, Muo CH, Chang YC, et al. Association between hypertensive disorders during pregnancy and end-stage renal disease: a populationbased study. CMAJ. 2013;185:207-213.

399. Beharier O, Shoham-Vardi I, Pariente G, et al. Gestational diabetes mellitus is a significant risk factor for long-term maternal renal disease. J Clin Endocrinol Metab. 2015;100:1412-1416.

400. The Royal College of Radiologists and the College of Radiographers Protection of Pregnant Patients during Diagnostic Medical Exposures to lonising Radiation Advice from the Health Protection Agency. Documents of the Health Protection Agency Radiation, Chemical and Environmental Hazards, 2009. https://www.rcr.ac.uk/sites/default/files/ publication/HPA_preg_2nd.pdf. (Accessed on September 8 2015).

401. Garg AX, Nevis IF, McArthur E, et al. Gestational hypertension and preeclampsia in living kidney donors. N Engl J Med. 2015;372:124-133.

402. Reisaeter AV, Roislien J, Henriksen T, et al. Pregnancy and birth after kidney donation: the Norwegian experience. Am J Transplant. 2009;9:820-824.

403. Ibrahim HN, Akkina SK, Leister E, et al. Pregnancy outcomes after kidney donation. Am J Transplant. 2009;9:825-834.

404. Thangaratinam S, Rogozińska E, Jolly K, et al. Interventions to reduce or prevent obesity in pregnant women: a systematic review. Health Technol Assess. 2012;16:iii-iv 1-191.

405. Allen R, Rogozinska E, Sivarajasingam P, et al. Effect of diet- and lifestylebased metabolic risk-modifying interventions on preeclampsia: a metaanalysis. Acta Obstet Gynecol Scand. 2014;93:973-985.

406. Rasmussen PE, Nielsen FR. Hydronephrosis during pregnancy: a literature survey. Eur J Obstet Gynecol Reprod Biol. 1988;27:249-259.

407. Garg AX, McArthur E, Lentine KL, et al. Gestational hypertension and preeclampsia in living kidney donors. N Engl J Med. 2015;372: 1469-1470.

408. Duerinckx N, Timmerman L, Van Gogh J, et al. Predonation psychosocial evaluation of living kidney and liver donor candidates: a systematic literature review. Transpl Int. 2014;27:2-18.

409. van Hardeveld E, Tong A. The CARI guidelines. Psychosocial care of living kidney donors. Nephrology (Carlton). 2010;15(Suppl 1): S80-S87.

410. Henderson AJ, Landolt MA, McDonald MF, et al. The living anonymous kidney donor: lunatic or saint? Am J Transplant. 2003;3:203-213.

411. Sharif A. Unspecified kidney donation-a review of principles, practice and potential. Transplantation. 2013;95:1425-1430.

412. Tong A, Craig JC, Wong G, et al. "It was just an unconditional gift." Self reflections of non-directed living kidney donors. Clin Transplant. 2012; 26:589-599.

413. Clemens K, Boudville N, Dew MA, et al. The long-term quality of life of living kidney donors: a multicenter cohort study. Am J Transplant. 2011;11:463-469.

414. Lentine KL, Schnitzler MA, Xiao H, et al. Depression diagnoses after living kidney donation: linking U.S. Registry data and administrative claims. Transplantation. 2012;94:77-83. 
415. Gross CR, Messersmith EE, Hong BA, et al. Health-related quality of life in kidney donors from the last five decades: results from the RELIVE study. Am J Transplant. 2013;13:2924-2934.

416. de Groot IB, Stiggelbout AM, van der Boog PJ, et al. Reduced quality of life in living kidney donors: association with fatigue, societal participation and pre-donation variables. Transpl Int. 2012;25:967-975.

417. Dew MA, DiMartini AF, DeVito Dabbs AJ, et al. Preventive intervention for living donor psychosocial outcomes: feasibility and efficacy in a randomized controlled trial. Am J Transplant. 2013;13:2672-2684.

418. Jowsey SG, Jacobs C, Gross CR, et al. Emotional well-being of living kidney donors: findings from the RELIVE Study. Am J Transplant. 2014;14:2535-2544.

419. Messersmith EE, Gross CR, Beil CA, et al. Satisfaction with life among living kidney donors: a RELIVE study of long-term donor outcomes. Transplantation. 2014;98:1294-1300.

420. Wirken L, van Middendorp H, Hooghof CW, et al. The course and predictors of health-related quality of life in living kidney donors: a systematic review and meta-analysis. Am J Transplant. 2015;15:3041-3054.

421. The Declaration of Istanbul on organ trafficking and transplant tourism. Transplantation 2008;86:1013-1018.

422. Thys K, Schwering KL, Siebelink M, et al. Psychosocial impact of pediatric living-donor kidney and liver transplantation on recipients, donors, and the family: a systematic review. Transpl Int. 2015;28:270-280.

423. Tong A, Chapman JR, Wong G, et al. The motivations and experiences of living kidney donors: a thematic synthesis. Am J Kidney Dis. 2012; 60:15-26.

424. Jacobs CL, Gross CR, Messersmith EE, et al. Emotional and financial experiences of kidney donors over the past 50 Years: The RELIVE study. Clin J Am Soc Nephrol. 2015;10:2221-2231.

425. Clemens KK, Thiessen-Philbrook H, Parikh CR, et al. Psychosocial health of living kidney donors: a systematic review. Am J Transplant. 2006:6:2965-2977.

426. Clarke KS, Klarenbach S, Vlaicu S, et al. The direct and indirect economic costs incurred by living kidney donors-a systematic review. Nephrol Dial Transplant. 2006;21:1952-1960.

427. Rodrigue JR, Schold JD, Morrissey P, et al. Direct and indirect costs following living kidney donation: findings from the KDOC study. Am J Transplant. 2016;16:869-876.

428. Klarenbach S, Gill JS, Knoll G, et al. Economic consequences incurred by living kidney donors: a Canadian multi-center prospective study. Am J Transplant. 2014;14:916-922.

429. Rodrigue JR, Schold JD, Morrissey P, et al. Predonation direct and indirect costs incurred by adults who donated a kidney: findings from the KDOC study. Am J Transplant. 2015;15:2387-2393.

430. Delmonico FL, Martin D, Dominguez-Gil B, et al. Living and deceased organ donation should be financially neutral acts. Am J Transplant. 2015;15:1187-1191.

431. Sickand M, Cuerden MS, Klarenbach SW, et al. Reimbursing live organ donors for incurred non-medical expenses: a global perspective on policies and programs. Am J Transplant. 2009:9:2825-2836.

432. Tushla L, Rudow DL, Milton J, et al. Living-donor kidney transplantation: reducing financial barriers to live kidney donation-recommendations from a Consensus Conference. Clin J Am Soc Nephrol. 2015;10:1696-1702.

433. Lacetera N, Macis M, Stith SS. Removing financial barriers to organ and bone marrow donation: the effect of leave and tax legislation in the U.S. $J$ Health Econ. 2014;33:43-56.

434. Boyarsky BJ, Massie AB, Alejo JL, et al. Experiences obtaining insurance after live kidney donation. Am J Transplant. 2014;14:2168-2172.

435. Yang RC, Thiessen-Philbrook H, Klarenbach S, et al. Insurability of living organ donors: a systematic review. Am J Transplant. 2007;7:1542-1551.

436. Yang RC, Young A, Nevis IF, et al. Life insurance for living kidney donors: a Canadian undercover investigation. Am J Transplant. 2009;9: 1585-1590.

437. Mittelman M, Thiessen $\mathrm{C}$, Chon WJ, et al. Miscommunicating NOTA can be costly to living donors. Am J Transplant. 2017;17:578-580.

438. American Society of Transplantation (AST). Live Donor Financial Toolkit. Available at: https://www.myast.org/patient-information/live-donortoolkit. (Accessed: September 7, 2016).

439. Frade IC, Fonseca I, Dias L, et al. Impact assessment in living kidney donation: psychosocial aspects in the donor. Transplant Proc. 2008;40: 677-681.

440. Giessing M, Reuter S, Schönberger B, et al. Quality of life of living kidney donors in Germany: a survey with the Validated Short Form-36 and Giessen Subjective Complaints List-24 questionnaires. Transplantation. 2004;78:864-872
441. Haljamae U, Nyberg G, Sjostrom B. Remaining experiences of living kidney donors more than 3 yr after early recipient graft loss. Clin Transplant. 2003; 17:503-510.

442. Johnson EM, Anderson JK, Jacobs C, et al. Long-term follow-up of living kidney donors: quality of life after donation. Transplantation. 1999;67:717-721.

443. Weizer N, Weizman A, Shapira Z, et al. Suicide by related kidney donors following the recipients' death. Psychother Psychosom. 1989;51:216-219.

444. Manyalich M, Ricart A, Martinez I, et al. EULID project: European living donation and public health. Transplant Proc. 2009;41:2021-2024.

445. Thiel GT, Nolte C, Tsinalis D. Prospective Swiss cohort study of livingkidney donors: study protocol. BMJ Open. 2011;1:e000202.

446. lacoviello BM, Shenoy A, Braoude J, et al. The live donor assessment tool: a psychosocial assessment tool for live organ donors. Psychosomatics. 2015;56:254-261.

447. Ismail SY, Duerinckx N, van der Knoop MM, et al. Toward a conceptualization of the content of psychosocial screening in living organ donors: an ethical legal psychological aspects of transplantation consensus. Transplantation. 2015;99:2413-2421.

448. Fonouni H, Mehrabi A, Golriz M, et al. Comparison of the laparoscopic versus open live donor nephrectomy: an overview of surgical complications and outcome. Langenbecks Arch Surg. 2014;399:543-551.

449. Liu N, Wazir R, Wang J, et al. Maximizing the donor pool: left versus right laparoscopic live donor nephrectomy - systematic review and metaanalysis. Int Urol Nephrol. 2014;46:1511-1519.

450. Wilson CH, Sanni A, Rix DA, et al. Laparoscopic versus open nephrectomy for live kidney donors. Cochrane Database Syst Rev. 2011:CD006124.

451. Yuan H, Liu L, Zheng S, et al. The safety and efficacy of laparoscopic donor nephrectomy for renal transplantation: an updated meta-analysis. Transplant Proc. 2013:45:65-76.

452. Tanaka M, Ono Y, Matsuda T, et al. Guidelines for urological laparoscopic surgery. Int J Urol. 2009;16:115-125.

453. Gibbons N, Nicol D, CARI. The CARI guidelines. Surgical techniques in living donor nephrectomy. Nephrology (Cartton). 2010;15(Suppl 1):S88-S95.

454. Dols LF, Kok NF, d'Ancona FC, et al. Randomized controlled trial comparing hand-assisted retroperitoneoscopic versus standard laparoscopic donor nephrectomy. Transplantation. 2014;97:161-167.

455. Cohen AJ, Williams DS, Bohorquez $\mathrm{H}$, et al. Robotic-assisted laparoscopic donor nephrectomy: decreasing length of stay. Ochsner J. 2015;15:19-24

456. Laplace B, Ladriere M, Claudon M, et al. Robotic assisted laparoscopic living donor nephrectomy: preoperative assessment and results of 100 cases. Prog Urol. 2014;24:288-293.

457. Tzvetanov I, Bejarano-Pineda L, Giulianotti PC, et al. State of the art of robotic surgery in organ transplantation. World J Surg. 2013;37:2791-2799.

458. Kaouk JH, Khalifeh A, Laydner $\mathrm{H}$, et al. Transvaginal hybrid natural orifice transluminal surgery robotic donor nephrectomy: first clinical application. Urology. 2012;80:1171-1175.

459. Alcaraz A, Musquera M, Peri L, et al. Feasibility of transvaginal natural orifice transluminal endoscopic surgery-assisted living donor nephrectomy: is kidney vaginal delivery the approach of the future? Eur Urol. 2011:59:1019-1025.

460. Kortram K, lizermans JN, Dor FJ. Perioperative events and complications in minimally invasive live donor nephrectomy: a systematic review and meta-analysis. Transplantation. 2016;100:2264-2275.

461. Gupta A, Ahmed K, Kynaston HG, et al. Laparoendoscopic single-site donor nephrectomy (LESS-DN) versus standard laparoscopic donor nephrectomy. Cochrane Database Syst Rev. 2016:CD010850

462. Khalil A, Muitaba MA, Taber TE, et al. Trends and outcomes in right vs. left living donor nephrectomy: an analysis of the OPTN/UNOS database of donor and recipient outcomes - should we be doing more right-sided nephrectomies? Clin Transplant. 2016;30:145-153.

463. Dols LF, Kok NF, Alwayn IP, et al. Laparoscopic donor nephrectomy: a plea for the right-sided approach. Transplantation. 2009;87:745-750.

464. Klop KW, Kok NF, Dols LF, et al. Can right-sided hand-assisted retroperitoneoscopic donor nephrectomy be advocated above standard laparoscopic donor nephrectomy: a randomized pilot study. Transp/ Int. 2014;27:162-169.

465. Apisarnthanarak P, Suvannarerg V, Muangsomboon K, et al. Renal vascular variants in living related renal donors: evaluation with CT angiography. J Med Assoc Thai. 2012;95:941-948.

466. Roza AM, Perloff LJ, Naji A, et al. Living-related donors with bilateral multiple renal arteries. A twenty-year experience. Transplantation. 1989;47: 397-399. 
467. Flechner SM, Sandler CM, Houston GK, et al. 100 living-related kidney donor evaluations using digital subtraction angiography. Transplantation. 1985;40:675-678.

468. Ahmadi AR, Lafranca JA, Claessens LA, et al. Shifting paradigms in eligibility criteria for live kidney donation: a systematic review. Kidney Int. 2015;87:31-45.

469. Friedman AL, Peters TG, Ratner LE. Regulatory failure contributing to deaths of live kidney donors. Am J Transplant. 2012;12:829-834.

470. Friedman AL, Peters TG, Jones KW, et al. Fatal and nonfatal hemorrhagic complications of living kidney donation. Ann Surg. 2006;243: 126-130.

471. Janki S, Verver D, Klop KW, et al. Vascular management during live donor nephrectomy: an online survey among transplant surgeons. Am J Transplant. 2015;15:1701-1707.

472. Serrano OK, Bangdiwala AS, Vock DM, et al. Defining the tipping point in surgical performance for laparoscopic donor nephrectomy among transplant surgery fellows: a risk-adjusted cumulative summation learning curve analysis. Am J Transplant. 2017;17:1868-1878.

473. European Union. Toolbox Living Kidney Donation. Developed by experts of the working group on living donation nominated by their National Competent Authorities (CAs) established by Directive 2010/53/EU)]. 2014. Available at: http://ec.europa.eu/health/blood_tissues_organs/docs/eutoolbox_living_ kidney_donation_en.pdf. (Accessed: September 7, 2016).

474. Lopp L. Regulations Regarding Living Organ Donation in Europe. Possibilities of Harmonisation. Springer-Verlag Berlin Heidelberg; 2013.

475. Lennerling A, Lovén C, Dor FJ, et al. Living organ donation practices in Europe — results from an online survey. Transpl Int. 2013;26:145-153.

476. Thys K, Van Assche K, Nobile H, et al. Could minors be living kidney donors? A systematic review of guidelines, position papers and reports. Transpl Int. 2013;26:949-960.

477. Van Assche K, Genicot G, Sterckx S. Living organ procurement from the mentally incompetent: the need for more appropriate guidelines. Bioethics. 2014;28:101-109.

478. Veatch R. Transplantation Ethics. Chapter 12: Live Donor Transplant. Georgetown University Press; 2000.

479. Tenenbaum EM. Bartering for a compatible kidney using your incompatible, live kidney donor: legal and ethical issues related to kidney chains. Am J Law Med. 2016;42:129-169.

480. Tong A, Ralph A, Chapman JR, et al. Public attitudes and beliefs about living kidney donation: focus group study. Transplantation. 2014;97: 977-985.

481. Young A, Karpinski M, Treleaven D, et al. Differences in tolerance for health risk to the living donor among potential donors, recipients, and transplant professionals. Kidney Int. 2008;73:1159-1166.

482. Rodrigue JR, Cornell DL, Kaplan B, et al. Patients' willingness to talk to others about living kidney donation. Prog Transplant. 2008;18:25-31.

483. Waterman AD, Morgievich M, Cohen DJ, et al. Living donor kidney transplantation: Improving education outside of transplant centers about live donor transplantation-recommendations from a consensus conference. Clin J Am Soc Nephrol. 2015;10:1659-1669.

484. OPTN (Organ Procurement and Transplantation Network)/UNOS (United Network for Organ Sharing): OPTN 2015 Strategic Plan. Available at: http://optn.transplant.hrsa.gov/governance/strategic-plan/. (Accessed: September 7, 2016).

485. Taylor M. White House gets major commitments on organ transplants, donations. June 13, 2016. Available at: http://medcitynews.com/2016/ 06/white-house-organ-transplants/. (Accessed: September 7, 2016).

486. Garonzik-Wang JM, Berger JC, Ros RL, et al. Live donor champion: finding live kidney donors by separating the advocate from the patient. Transplantation. 2012;93:1147-1150.

487. Rodrigue JR, Pavlakis M, Egbuna O, et al. The "House Calls" trial: a randomized controlled trial to reduce racial disparities in live donor kidney transplantation: rationale and design. Contemp Clin Trials. 2012;33:811-818.

488. Rodrigue JR, Cornell DL, Lin JK, et al. Increasing live donor kidney transplantation: a randomized controlled trial of a home-based educational intervention. Am J Transplant. 2007;7:394-401.

489. Ismail SY, Luchtenburg AE, Timman R, et al. Home-based family intervention increases knowledge, communication and living donation rates: a randomized controlled trial. Am J Transplant. 2014;14:1862-1869.

490. Waterman AD, Robbins ML, Peipert JD. Educating prospective kidney transplant recipients and living donors about living donation: practical and theoretical recommendations for increasing living donation rates. Curr Transplant Rep. 2016;3:1-9.
491. Kumar K, King EA, Muzaale AD, et al. A smartphone app for increasing live organ donation. Am J Transplant. 2016;16:3548-3553.

492. Erickson J, Barbero R, Brewster M. A call to action to help reduce the waiting list for organ transplants. 2016. Available at: https://www. whitehouse.gov/blog/2016/09/01/call-action-help-reduce-waiting-listorgan-transplants. (Accessed: September 7, 2016).

493. Allen MB, Reese PP. The ethics of promoting living kidney donation using nonargumentative influence: applications, concerns, and future directions. Am J Transplant. 2016;16:3378-3384.

494. Wright L, Faith K, Richardson R, et al. Ethical guidelines for the evaluation of living organ donors. Can J Surg. 2004;47:408-413.

495. Frunza M, Van Assche K, Lennerling A, et al. Dealing With public solicitation of organs from living donors-an ELPAT view. Transplantation. 2015;99:2210-2214.

496. Wright L. Ethical controversies in public solicitations for organs. Transplant Rev (Orlando). 2008;22:184-186.

497. Neidich EM, Neidich AB, Cooper JT, et al. The ethical complexities of online organ solicitation via donor-patient websites: avoiding the "beauty contest." Am J Transplant. 2012;12:43-47.

498. International Summit on Transplant Tourism and Organ Trafficking. The Declaration of Istanbul on organ trafficking and transplant tourism. Kidney Int 2008;74:854-859.

499. Andrews PA, Burnapp L, Manas D, et al. Summary of the British Transplantation Society/Renal Association U.K. guidelines for living donor kidney transplantation. Transplantation. 2012;93:666-673.

500. Hays R, Rodrigue JR, Cohen D, et al. Financial neutrality for living organ donors: reasoning, rationale, definitions, and implementation strategies. Am J Transplant. 2016;16:1973-1981.

501. NOTA. National Organ Transplant Act. 42 U.S.C. § 273 §274. 1984 https://history.nih.gov/research/downloads/PL98-507.pdf. Accessed January 24, 2017.

502. LaPointe Rudow D, Cohen D. Practical approaches to mitigating economic barriers to living kidney donation for patients and programs. Curr Transpl Rep. 2017;4:24-31

503. Radio New Zealand. New law gives financial assistance to organ donors. http://www.radionz.co.nz/news/political/319407/new-law-gives-financial-assistance-to-organ-donors. (Accessed on Jan 14, 2017).

504. American Society of Transplant Surgeons. Lost Wages Reimbursement for Living Organ Donors Trial Announced. https://asts.org/newsand-publications/asts-news/article/2016/12/21/lost-wages-reimbursement-for-living-organ-donors-trial-announced\#WHqB1H05BQp. (Accessed January 14, 2017).

505. Gaston RS, Danovitch GM, Epstein RA, et al. Limiting financial disincentives in live organ donation: a rational solution to the kidney shortage. Am J Transplant. 2006;6:2548-2555.

506. Joshi S, Kupin W. Reciprocating living kidney donor generosity: tax credits, health insurance and an outcomes registry. Clin Kidney $\mathrm{J}$. 2016;9:168-171.

507. Congressional Research Service. Summaries for the Living Donor Protection Act of 2016. Available at: https://www.govtrack.us/congress/ bills/114/hr4616/summary. (Accessed: September 7, 2016).

508. Gill JS, Delmonico F, Klarenbach S, et al. Providing coverage for the unique lifelong health care needs of living kidney donors within the framework of financial neutrality. Am J Transplant. 2017;17:1176-1181.

510. Matas AJ. The rationale for incentives for living donors: an international perspective? Curr Transpl Rep. 2015;2:44-51.

511. Wright L, Anstey A. Contact Between Living Anonymous Donors and Recipients: Ethical Issues. Ethical, Legal and Psychological Aspects of Transplantation. Global Issues, Local Solutions. Weimar W, Bos MA, van Busschbach JJ: eds.). Lengerich, Germany: Pabst Publishers; 2014.

512. OPTN (Organ Procurement and Transplantation Network)/UNOS (United Network for Organ Sharing). OPTN Policies, Policy 8: Allocation of Kidneys. Available at: http://optn.transplant.hrsa.gov/governance/policies. (Accessed: June 28, 2017).

513. Muzaale AD, Massie AB, Kucirka LM, et al. Outcomes of live kidney donors who develop end-stage renal disease. Transplantation. 2016; 100:1306-1312.

514. Potluri V, Harhay MN, Wilson FP, et al. Kidney transplant outcomes for prior living organ donors. J Am Soc Nephrol. 2015;26:1188-1194.

515. Stewart DE, Kucheryavaya AY, Klassen DK, et al. Changes in deceased donor kidney transplantation one year after KAS implementation. Am J Transplant. 2016;16:1834-1847.

516. Wainright JL, Klassen DK, Kucheryavaya AY, et al. Delays in prior living kidney donors receiving priority on the transplant waiting list. Clin J Am Soc Nephrol. 2016;11:2047-2052. 
517. Lavee J, Ashkenazi T, Gurman G, et al. A new law for allocation of donor organs in Israel. Lancet. 2010;375:1131-1133.

518. Lavee J, Ashkenazi T, Stoler A, et al. Preliminary marked increase in the national organ donation rate in Israel following implementation of a new organ transplantation law. Am J Transplant. 2013;13: 780-785.

519. Tumin M, Rasiah R, Noh A, et al. Living kidney donation: the importance of public education. Clin Transplant. 2014;28:423-427.

520. Salomon DR, Langnas AN, Reed Al, et al. AST/ASTS workshop on increasing organ donation in the United States: creating an "arc of change" from removing disincentives to testing incentives. Am J Transplant. 2015;15:1173-1179.

521. Tong A, Chapman JR, Wong G, et al. Perspectives of transplant physicians and surgeons on reimbursement, compensation, and incentives for living kidney donors. Am J Kidney Dis. 2014;64: 622-632.

522. Mandelbrot DA, Pavlakis M, Karp SJ, et al. Practices and barriers in longterm living kidney donor follow-up: a survey of U.S. transplant centers. Transplantation. 2009;88:855-860.

523. Leichtman A, Abecassis M, Barr M, et al. Living kidney donor follow-up: state-of-the-art and future directions, conference summary and recommendations. Am J Transplant. 2011;11:2561-2568.

524. Van Assche K, Sterckx S, Lennerling A, et al. The relevance of Directive 2010/53/EU for living organ donation practice: an ELPAT view. Transplantation. 2015;99:2215-2222.

525. OPTN/UNOS Living Donor Committee. Procedures to collect postdonation follow-up data from living donors. Available at: https://optn. transplant.hrsa.gov/resources/guidance/procedures-to-collect-post-donationfollow-up-data-from-living-donors/. (Accessed: Septebmer 7, 2015).

526. Litwin MS. Editorial comment: the psychosocial impact of donating a kidney. J Urol. 1997;157:1600-1601.

527. Manyalich M, Menjivar A, Yucetin L, et al. Living donor psychosocial assessment/follow-up practices in the partners' countries of the ELIPSY project. Transplant Proc. 2012;44:2246-2249.
528. Weng FL, Reese PP, Waterman AD, et al. Health care follow-up by live kidney donors more than three yr post-nephrectomy. Clin Transplant. 2012;26:E300-E306

529. Reith P. Adapting the selective periodic health exam to a college-aged population. Joint American Preventive Services Task Force. Canadian Task Force on Health Care Screening. J Am Coll Health. 1989;38:109-113.

530. Schold JD, Buccini LD, Rodrigue JR, et al. Critical factors associated with missing follow-up data for living kidney donors in the United States. Am J Transplant. 2015;15:2394-2403.

531. Ommen ES, LaPointe Rudow D, Medapalli RK, et al. When good intentions are not enough: obtaining follow-up data in living kidney donors. Am J Transplant. 2011;11:2575-2581.

532. Davis CL. Living kidney donor follow-up: state-of-the-art and future directions. Adv Chronic Kidney Dis. 2012;19:207-211.

533. Casagrande LH, Collins S, Warren AT, et al. Lack of health insurance in living kidney donors. Clin Transplant. 2012;26:E101-E104.

534. Rodrigue JR, Fleishman A. Health insurance trends in United States living kidney donors (2004 to 2015). Am J Transplant. 2016;16:3504-3511.

535. Wainright J, McBride M, Dew MA, et al. Follow-up reporting for living kidney donors. Abstr 195. Am J Transplant. 2013;13:90.

536. Keshvani N, Feurer ID, Rumbaugh E, et al. Evaluating the Impact of performance improvement initiatives on transplant center reporting compliance and patient follow-up after living kidney donation. Am J Transplant. 2015;15:2126-2135.

537. Scientific Registry of Transplant Recipients. The Living Donor Collective: SRTR to Launch a Pilot Project to Create a Registry of Living Donors. https://www.srtr.org/news-media/news/news-items/news/. (Accessed June 28, 2017).

538. Lentine KL, Segev DL. Better understanding live donor risk through big data. Clin J Am Soc Nephrol. 2013;8:1645-1647.

539. Kulkarni S, Thiessen C, Formica RN, et al. The long-term follow-up and support for living organ donors: A center-based initiative founded on developing a community of living donors. Am J Transplant. 2016;16:3385-3391. 